\title{
New Mid-IR Lasers Based on Rare-Earth Doped Sulfide and Chloride Materials
}

\author{
M. C. Nostrand \\ Ph.D. Dissertation
}

September 1, 2000

U.S. Department of Energy

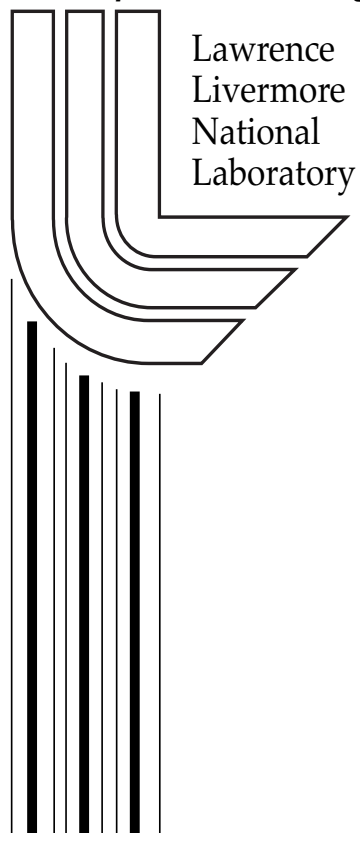




\section{DISCLAIMER}

This document was prepared as an account of work sponsored by an agency of the United States Government. Neither the United States Government nor the University of California nor any of their employees, makes any warranty, express or implied, or assumes any legal liability or responsibility for the accuracy, completeness, or usefulness of any information, apparatus, product, or process disclosed, or represents that its use would not infringe privately owned rights. Reference herein to any specific commercial product, process, or service by trade name, trademark, manufacturer, or otherwise, does not necessarily constitute or imply its endorsement, recommendation, or favoring by the United States Government or the University of California. The views and opinions of authors expressed herein do not necessarily state or reflect those of the United States Government or the University of California, and shall not be used for advertising or product endorsement purposes.

This work was performed under the auspices of the U. S. Department of Energy by the University of California, Lawrence Livermore National Laboratory under Contract No. W-7405-Eng-48.

This report has been reproduced directly from the best available copy.

Available electronically at http://www.doe.gov/bridge

Available for a processing fee to U.S. Department of Energy

and its contractors in paper from

U.S. Department of Energy

Office of Scientific and Technical Information

P.O. Box 62

Oak Ridge, TN 37831-0062

Telephone: (865) 576-8401

Facsimile: (865) 576-5728

E-mail: reports@adonis.osti.gov

Available for the sale to the public from

U.S. Department of Commerce

National Technical Information Service

5285 Port Royal Road

Springfield, VA 22161

Telephone: (800) 553-6847

Facsimile: (703) 605-6900

E-mail: orders@ntis.fedworld.gov

Online ordering: http://www.ntis.gov/ordering.htm

\section{OR}

Lawrence Livermore National Laboratory

Technical Information Department's Digital Library

http:/ / www.llnl.gov/tid/Library.html 


\title{
FINAL DRAFT
}

3/8/2001 4:44 PM

New Mid-IR Lasers Based on Rare-Earth-Doped Sulfide and Chloride Materials

\author{
Mike Nostrand \\ (Ph.D. Dissertation)
}




\title{
New Mid-IR Lasers Based on Rare-Earth-Doped Sulfide and Chloride Materials
}

\author{
By \\ MICHAEL CRAIG NOSTRAND \\ B.S. (University of California, Los Angeles) 1988 \\ M.S. (San Francisco State University) 1994 \\ DISSERTATION \\ Submitted in partial satisfaction of the requirements for the degree of \\ DOCTOR OF PHILOSOPHY \\ in \\ APPLIED SCIENCE \\ in the \\ OFFICE OF GRADUATE STUDIES \\ of the \\ UNIVERSITY OF CALIFORNIA \\ DAVIS
}

Approved:

Denise M. Krol

Richard R. Freeman

Ralph H. Page 


\section{Acknowledgments}

'Acknowledge' seems a trivial concept when I reflect on the people that have supported me in the course of this dissertation. Colleagues, friends, and family have all contributed in an immeasurable way to this work. I am grateful to my committee for taking their valuable time to review this dissertation.

Of the many colleagues that have contributed to this work, Dr. Ralph H. Page must be singled out. His tireless dedication to my education could serve as a model for future advisors. His patience and expertise are greatly appreciated. I am grateful to Prof. Denise Krol for taking me on as a graduate student and providing me with much needed guidance and support. Dr. Kathleen I. Schaffers was of invaluable importance in my education of crystal growth and purification. She and her masterful assistant, John B. Tassano, were a large part of the success of this project. Also to be commended is Dr. Stephen A. Payne, whose guidance and vision always kept this project on high ground. The project first took flight in the mind of Dr. William F. Krupke, for which I am very grateful. The support and helpful conversations with many all the members of the Advanced Lasers and Components group are greatly appreciated.

This project depended heavily on collaborative efforts, especially in the area of crystal growth. My deepest thanks are extended to Pete Schunemann at Sanders for his efforts in producing quality crystals of $\mathrm{CaGa}_{2} \mathrm{~S}_{4}$ and $\mathrm{CaGa}_{2} \mathrm{Se}_{4}$, and to Ludmila Isaenko and A. Yelisseyev at the Design and Technological Institute for Monocystals for her amazing accomplishments in the growth of $\mathrm{KPb}_{2} \mathrm{Cl}_{5}$ and $\mathrm{RbPb}_{2} \mathrm{Cl}_{5}$. Also help were Peter Thelin for his polishing efforts and Terry Duewer for her concentration measurements. 
For making the scholastic experience a positive one I would like to thank my fellow DAS students, including my office mates Falgun D. Patel, Andy J. Bayramian, and John J. Adams.

Finally, I wish to thank my family for the confidence they maintained in me. If I have succeeded, it is because I have stood on the patience and sacrifices of my wife, Kristen. She has endured much, including the birth of our son Ryan and the growth of our daughter Jessica, during the course of this dissertation. I am very grateful to her and hope one day she will realize it was all 'worth it'. 


\section{Table of Contents}

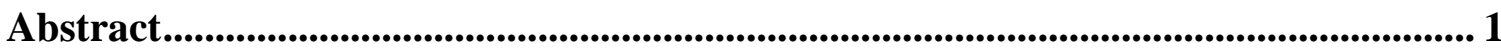

List of Publications ............................................................................................................... 3

List of Figures............................................................................................................................. 4

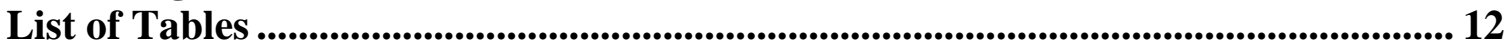

1 Introduction............................................................................................14

1.1 Motivation and Scope .......................................................................... 1.1-14

1.2 Low-Phonon-Frequency Hosts .............................................................. 1.2-18

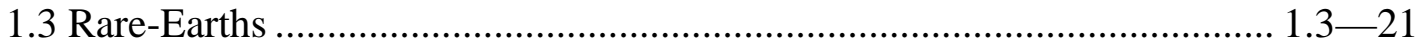

2 Theoretical Background............................................................................... 1.3-28

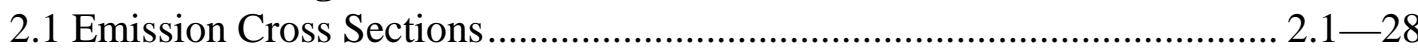

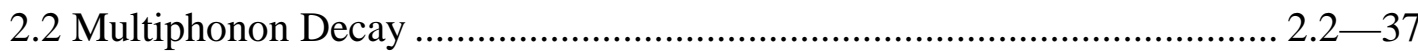

2.3 Nonradiative Energy Transfer ……………………............................. 2.3 - 42

2.4 Judd-Ofelt Analysis ........................................................................ 2.4 49

2.5 Laser Modeling ............................................................................... 2.5-52

2.6 Laser Predictions and Analysis............................................................ 2.6-57

3 Theoretical Investigations ........................................................................ 2.6-64

3.1 Quantum Efficiency Model ................................................................... 3.1-64

3.2 Emission cross section from laser performance analysis.......................... 3.2 $3-70$

3.3 Wedge in the Sample ........................................................................ 3.3-73

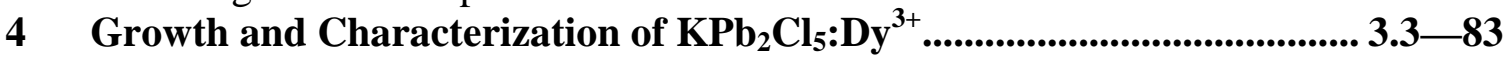

4.1 Sample Preparation ......................................................................... 4.1-83

4.2 Crystal Properties.............................................................................. 4.2-84

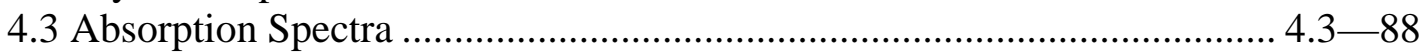

4.4 Judd-Ofelt data....................................................................................4-91

4.5 Decay rates and Energy Transfer........................................................ 4.5-92

4.6 Emission Data ............................................................................... 4.6-96

4.7 Quantum Efficiencies ....................................................................... 4.7-98

4.8 Other Chloride Samples..................................................................... 4.8-107

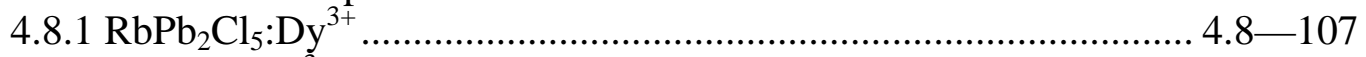

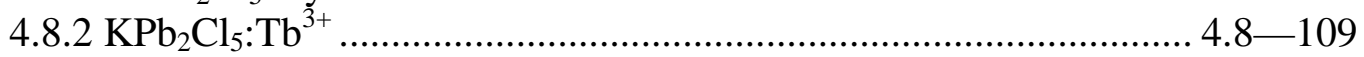

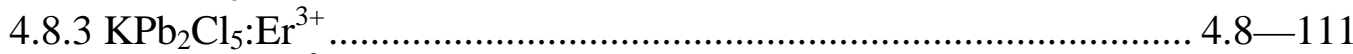

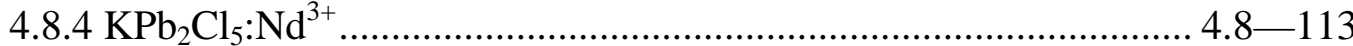

4.9 Multiphonon decay vs. Energy Gap ………………........................ 4.9-117

4.10 Conclusions..................................................................................... 4.10-118

$5 \quad \mathrm{KPb}_{2} \mathrm{Cl}_{5}$ Laser Results.......................................................................... 4.10_120

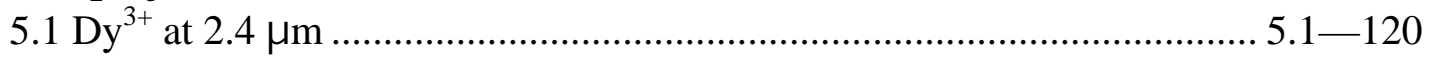

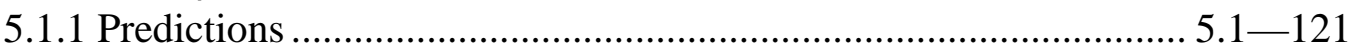

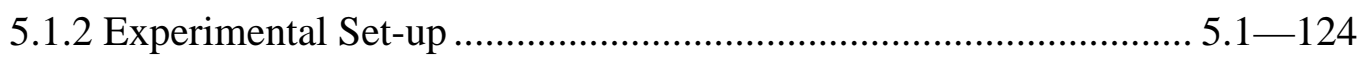

5.1.3 Laser Data and Analysis ............................................................ 5.1-125

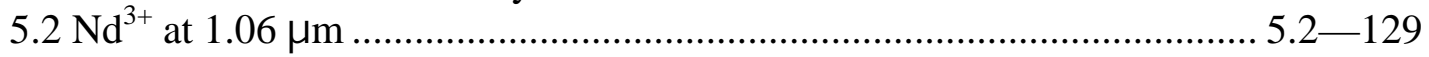

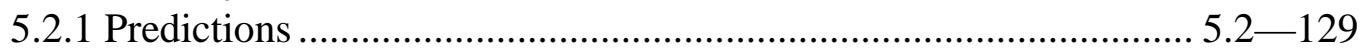

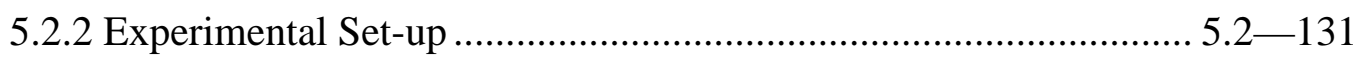

5.2.3 Laser Data and Analysis ............................................................. 5.2-131

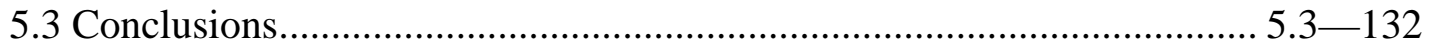




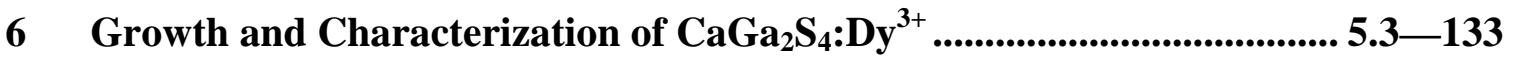

6.1 Sample Preparation ........................................................................... 6.1-133

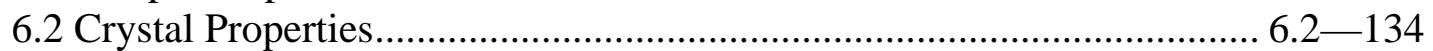

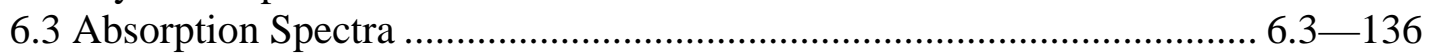

6.4 Judd-Ofelt Data.................................................................................... 6.4-139

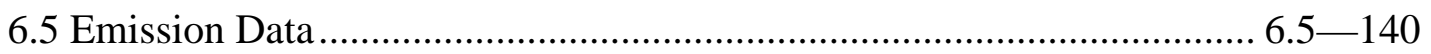

6.6 Quantum Efficiencies ...................................................................... 6.6-141

6.7 Other Chalcogenide Samples......................................................... 6.6.142

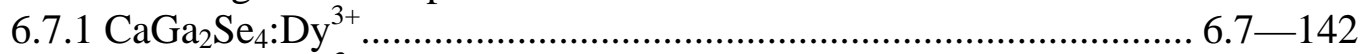

6.7.2 GeGaAsS:Dy ${ }^{3+}$ glass ........................................................... 6.7-145

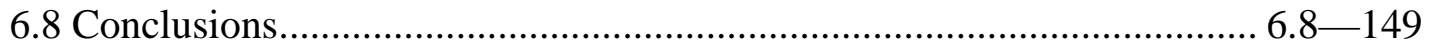

$7 \quad \mathrm{CaGa}_{2} \mathrm{~S}_{4}$ Laser Results ............................................................................. 6.8-150

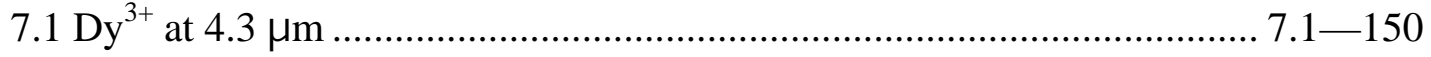

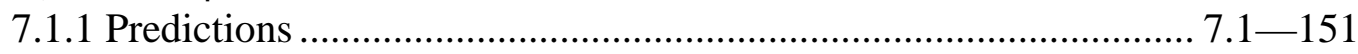

7.1.2 Experimental Set-up ............................................................. 7.1-153

7.1.3 Laser Data and Analysis.......................................................... 7.1-154

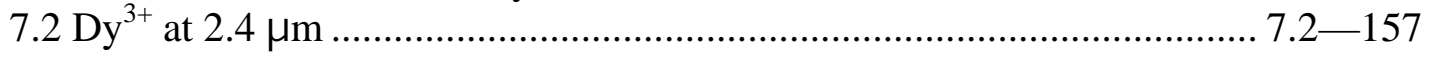

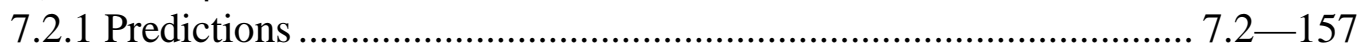

7.2.2 Experimental Set-up .............................................................. $7.2-159$

7.2.3 Laser Data and Analysis......................................................... 7.2-159

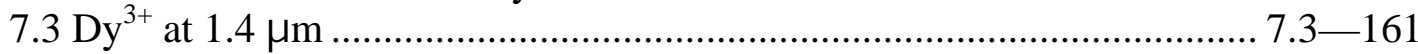

7.3.1 Predictions .............................................................................. $7.3-162$

7.3.2 Experimental Set-up .............................................................. $7.3-164$

7.3.3 Laser Data and Analysis .......................................................... 7.3-165

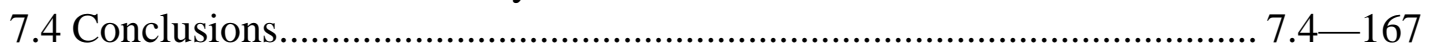

8 Summary .............................................................................................................................. 168

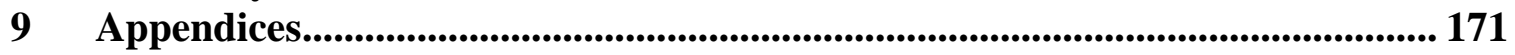

Appendix A : Derivation of integrated cross section relation ............................ 171

Appendix B : Local field correction in dielectrics ........................................... 173

Appendix C : Calculations of $S^{M D}$ for various rare-earth ions ......................... 175

Appendix D : Refractive index based on transmission measurements.............. 183

Appendix E : IR cut-off frequency and maximum phonon frequency .............. 185

Appendix F : Lock-in calibration factor......................................................... 187

Appendix G : Spot size measurements using the razor-scan technique ............ 189

10 References................................................................................................................. 191 


\section{Abstract}

Applications in remote-sensing and military countermeasures have driven a need for compact, solid-state mid-IR lasers. Due to multi-phonon quenching, non-traditional hosts are needed to extend current solid-state, room-temperature lasing capabilities beyond $\sim 4$ $\mu \mathrm{m}$. Traditional oxide and fluoride hosts have effective phonon energies in the neighborhood of $1000 \mathrm{~cm}^{-1}$ and $500 \mathrm{~cm}^{-1}$, respectively. These phonons can effectively quench radiation above 2 and $4 \mu \mathrm{m}$, respectively. Materials with lower effective phonon energies such as sulfides and chlorides are the logical candidates for mid-IR $(4-10 \mu \mathrm{m})$ operation.

In this report, laser action is demonstrated in two such hosts, $\mathrm{CaGa}_{2} \mathrm{~S}_{4}$ and $\mathrm{KPb}_{2} \mathrm{Cl}_{5}$. The $\mathrm{CaGa}_{2} \mathrm{~S}_{4}: \mathrm{Dy}^{3+}$ laser operating at $4.3 \mu \mathrm{m}$ represents the first sulfide laser operating beyond $2 \mu \mathrm{m}$. The $\mathrm{KPb}_{2} \mathrm{Cl}_{5}: \mathrm{Dy}^{3+}$ laser operating at $2.4 \mu \mathrm{m}$ represents the first operation of a chloride-host laser in ambient conditions. Laser action is also reported for $\mathrm{CaGa}_{2} \mathrm{~S}_{4}: \mathrm{Dy}^{3+}$ at $2.4 \mu \mathrm{m}, \mathrm{CaGa}_{2} \mathrm{~S}_{4}: \mathrm{Dy}^{3+}$ at $1.4 \mu \mathrm{m}$, and $\mathrm{KPb}_{2} \mathrm{Cl}_{5}: \mathrm{Nd}^{3+}$ at $1.06 \mu \mathrm{m}$.

Both host materials have been fully characterized, including lifetimes, absorption and emission cross sections, radiative branching ratios, and radiative quantum efficiencies. Radiative branching ratios and radiative quantum efficiencies have been determined both by the Judd-Ofelt method (which is based on absorption measurements), and by a novel method described herein which is based on emission measurements. Modeling has been performed to predict laser performance, and a new method to determine emission cross section from slope efficiency and threshold data is developed.

With the introduction and laser demonstration of rare-earth-doped $\mathrm{CaGa}_{2} \mathrm{~S}_{4}$ and $\mathrm{KPb}_{2} \mathrm{Cl}_{5}$, direct generation of mid-IR laser radiation in a solid-state host has been 
demonstrated. In $\mathrm{KPb}_{2} \mathrm{Cl}_{5}$, predictions indicate that laser operation to $9 \mu \mathrm{m}$ may be possible, a wavelength previously considered unreachable in a room-temperature, solidstate host. 


\section{List of Publications}

1. "Optical Properties and Laser Action for Rare-Earth-Doped $\mathrm{KPb}_{2} \mathrm{Cl}_{5}$ " M.C. Nostrand, R.H. Page, S.A. Payne, L.I. Isaenko, and A.P. Yelisseyev, J. Opt. Soc. B (to be published)

2. "Room-temperature laser action at 4.3-4.4 $\mu \mathrm{m}$ in $\mathrm{CaGa}_{2} \mathrm{~S}_{4}: \mathrm{Dy}^{3+}, "$ M.C. Nostrand, R.H. Page, S.A. Payne, W.F. Krupke, and P.G. Schunemann, Optics Letters 24, 1215 (1999)

3. "Dy ${ }^{3+}$-doped crystals of double chlorides and double fluorides as the active media of IR solid-state lasers and telecommunication amplifiers," A.M. Tkachuk, S.E. Ivanova, L.I. Isaenko, A.P. Eliseev, W.F. Krupke, S. A. Payne, R.W. Solarz, M.C. Nostrand, and R.H. Page, J. Opt. Technol., 66, 460 (1999)

4. "Quantum efficiency measurements for long-wavelength operation of rare-earthdoped $\mathrm{KPb}_{2} \mathrm{Cl}_{5}$," M.C. Nostrand, R.H. Page, S.A. Payne, W.F. Krupke, and L.I. Isaenko, Conference on Lasers and Electro-Optics (CLEO), May, 2000, paper CFA5

5. "Laser Demonstrations of Rare-Earth Ions in Low-Phonon Chloride and Sulfide Crystals", M.C. Nostrand, R.H. Page, S.A. Payne, W.F. Krupke, P.G. Schunemann and L.I. Isaenko, Advanced Solid-State Lasers (ASSL), February, 2000, paper WD4

6. "Spectroscopic data and mid-IR room-temperature laser action in $\mathrm{Dy}^{3+}$ and $\mathrm{Nd}^{3+}$ doped $\mathrm{KPb}_{2} \mathrm{Cl}_{5}$," M.C. Nostrand, R.H. Page, S.A. Payne, W.F. Krupke, A. Tkachuk, S. Ivanova, L. Isaenko, and A. Yelisseyev, $18^{\text {th }}$ Congress of the International Commission for Optics (ICO), August, 1999, paper 3749-308

7. "Room-temperature laser action at $4.38 \mu \mathrm{m}$ in $\mathrm{CaGa}_{2} \mathrm{~S}_{4}: \mathrm{Dy}^{3+}$, , M.C. Nostrand, R.H. Page, S.A. Payne, W.F. Krupke, and P.G. Schunemann, Conference on Lasers and Electro-Optics (CLEO), May, 1999, paper CThJ1

8. "Room-temperature $\mathrm{CaGa}_{2} \mathrm{~S}_{4}: \mathrm{Dy}^{3+}$ laser action at 2.43 and $4.31 \mu \mathrm{m}$ and $\mathrm{KPb}_{2} \mathrm{Cl}_{5}: \mathrm{Dy}^{3+}$ laser action at $2.43 \mu \mathrm{m}$," M.C. Nostrand, R.H. Page, S.A. Payne, W.F. Krupke, P.G. Schunemann, and L.I. Isaenko, OSA TOPS Vol. 26, Advanced SolidState Lasers (ASSL), 1999, page 441

9. "Spectroscopic data for infrared transitions in $\mathrm{CaGa}_{2} \mathrm{~S}_{4}: \mathrm{Dy}^{3+}$ and $\mathrm{KPb}_{2} \mathrm{Cl}_{5}$ :Dy ${ }^{3+}$," M.C. Nostrand, R.H. Page, S.A. Payne, W.F. Krupke, P.G. Schunemann, and L.I. Isaenko, OSA TOPS Vol. 19, Advanced Solid-State Lasers (ASSL), 1998, page 524

10. "Low-Phonon Frequency Chalcogenide Crystalline Hosts for Rare Earth Lasers Operating Beyond Three Microns", S.A. Payne, R.H. Page, M.C. Nostrand, K.I. Schaffers, and W.F. Krupke, U.S. Patent 6,047,013 (2000) 


\section{List of Figures}

Figure 1.1. Trivalent rare-earth energy levels below 22,000 $\mathrm{cm}^{-1}$. RussellSaunders ground-state labels are indicated. (data taken from Ref. 15).

Figure 1.2. Partial energy level diagram (not to scale) indicating the effect of the different interaction Hamiltonians on $\mathrm{Pr}^{3+}$. The spin-orbit interaction gives rise to the Russell-Saunders levels indicated in Figure 1.1. The crystal-field Hamiltonian splits these levels into Stark levels. (Taken from Ref. [30])

Figure 2.1. Illustration of concepts used to determine the emission cross section. The symbols are defined in the text.

Figure 2.2. Plot showing the relationship between the radiative branching ratio $\beta_{21}$ and the wavelength $\lambda$ that conserves the emission cross section $\sigma^{e m}$, all other factors being equal.

Figure 2.3. Schematic of the concepts used in this section. The upper manifold has a partition function $Z_{u}$, and has $g_{u}$ states associated with it, each with energy $E_{j}$ (measured relative to the lowest level in the manifold) and Boltzmann occupation factor $f_{j}$. Similar statements are true of the lower manifold.

Figure 2.4. Example of an emission and absorption spectrum at different temperatures. At low temperatures (near $0 \mathrm{~K}$ ), only the lowest ground-state Stark level is populated, and absorption transitions will originate only from this level. At higher temperatures, more Stark levels are populated so that more transitions can take place. This broadens the spectrum considerably. The area under the spectra are conserved. A similar process applies to the emission spectrum.

Figure 2.5. multiphonon decay (at temperature $T$ ) is characterized by a decay rate $W^{M P}$, an energy gap $\Delta \mathrm{E}$, and an effective phonon energy $\hbar \omega_{\text {eff }}$

Figure 2.6. Multiphonon emission rate vs. energy gap for various hosts. Effective phonon energies are shown in parentheses. (From )....

Figure 2.7. Energy is transferred by the nonradiative interaction $H^{\prime}$ from the donor, initially in state $\left|D^{*}\right\rangle$, to the acceptor, initially in state $|A\rangle$. The donor $\mathrm{D}$ and acceptor $\mathrm{A}$ are separated by a distance $\mathrm{R}$. (after Ref. 30)

Figure 2.8. Decay curves for the various cases described in the text. (a) represents $\phi_{1}\left(\mathrm{~A}^{\mathrm{rad}} \mathrm{t}\right)$ for $\mathrm{W}^{0}=\mathrm{A}^{\mathrm{rad}}$. (b) represents $\phi_{2}\left(\mathrm{~A}^{\mathrm{rad}} \mathrm{t}\right)$ with $\mathrm{N}_{\mathrm{A}} \mathrm{R}_{\mathrm{DA}}{ }^{3}=1$. (c) represents $\phi_{3}\left(\mathrm{~A}^{\mathrm{rad}} \mathrm{t}\right)$ with $\mathrm{N}_{\mathrm{A}} \mathrm{R}_{\mathrm{DA}}{ }^{3}=1$ and $\mathrm{N}_{\mathrm{D}} \mathrm{R}_{\mathrm{DD}}{ }^{3}=0.1$. (d) represents $\phi_{4}\left(\mathrm{~A}^{\mathrm{rad}} \mathrm{t}\right)$ with $\mathrm{N}_{\mathrm{A}} \mathrm{R}_{\mathrm{DA}}{ }^{3}=1$ and $\mathrm{N}_{\mathrm{D}} \mathrm{R}_{\mathrm{DD}}^{3}=3$. $2.3-48$

Figure 2.9. The Stokes cross section manifests itself as the exponential tail of the electronic cross section. Note $\sigma^{\text {Stokes }}$ appears as a straight line on the log-scale plot. 
Figure 2.10. Schematic of the energy-level diagram used in the temporal modeling of laser output. Laser action occurs between individual Stark levels with populations $\mathrm{N}_{2}$ and $\mathrm{N}_{1}$. Each manifold is assumed to be in thermal equilibrium. The upper and lower manifold have populations $\mathrm{N}_{\mathrm{U}}$ and $\mathrm{N}_{\mathrm{L}}$, respectively.....

Figure 2.11. Numerically generated plot of the normalized slope efficiency $d S / d F$ as a function of input power $\mathrm{P}_{\text {in }} / \mathrm{P}_{\text {th }}$. Note $d S / d F \approx 1$ for $a<$ 0.5 . The parameter $a$ is defined as $w_{p} / w_{l}$. $d S / d F$ is identified as $\eta_{\text {mode }}$ of Section 2.5.

Figure 3.1. Energy level diagram of $\mathrm{Dy}^{3+}: \mathrm{KPb}_{2} \mathrm{Cl}_{5}$. The letter designations of the levels are taken after Dieke [15]. The phonon-assisted energy transfer pathway is indicated. Note this pathway actually involves two neighboring ions: a donor ion in the W-level and an acceptor ion in the Z-level interacting to promote both ions into the Y-level....

Figure 3.2. Pedagogical illustration of the concept of the radiative quantum efficiency model. a) Level 3 is pumped directly, and we assume $\eta_{2}^{\text {rad }}=1$ and $\beta_{32}^{\text {rad }}=0$. The wavy arrow indicates nonradiative decay. Hypothetical emission rates $\phi$ (arb. photons/sec) are shown as a function of wavelength $\lambda$ for the cases b) $\eta_{3}^{\text {rad }}=1$, c) $\eta_{3}^{\text {rad }}=0.5$, and d) $\eta_{3}^{\text {rad }}=0$.

Figure 3.3. Notation used in this section. The pre-superscript refers to the level that was pumped and the post-subscript refers to the emitting level. The post-superscript refers to the nature of the process involved.

Figure 3.4. Illustration of the threshold-slope product. As evidenced by the equation, the negative of the threshold-slope product is the intercept of the slope-efficiency curve at zero input. This remains independent of intracavity losses $L$ since as these losses are increased, the slope-efficiency curve simply pivots about the intercept.

Figure 3.5. The threshold-slope product vs. transmission in $\mathrm{Nd}: \mathrm{YAG}$ operating at $1.064 \mu \mathrm{m}$. The line represents the least squares fit to the data with intercept at the origin, according to (3.20).

Figure 3.6. A ray is characterized by is distance from the optic axis $r$ and its angle with the axis $\theta$.

Figure 3.7. Schematic of the propagation modes inside the cavity. The sample has index $n$, wedge angle $\alpha$, and axial length $l$. The optic axis intersects the sample a distance $h$ from the lower edge. $d_{1}$ and $d_{2}$ are the optical distances from the mirror to each sample surface, and the mirrors have reflectivities $\mathrm{R}_{1}$ and $\mathrm{R}_{2}$, as shown.

Figure 3.8. Geometry of a ray passing through a wedged sample. The sample wedge angle $\alpha$ is shown exaggerated for clarity. 
Figure 3.9. Ray-tracing output for two round-trips in a $20-\mathrm{cm}$ confocal cavity $(\mathrm{L}=\mathrm{R}=20 \mathrm{~cm})$. The wedge angle is $3 \mathrm{mrad}$, the crystal length is 1 $\mathrm{cm}$, and the ray is launched on-axis at $\mathrm{r}=0$, starting from the left cavity mirror (of radius $R$ ).

Figure 3.10. Ray tracing output for a symmetric cavity with $R=20 \mathrm{~cm}$ for varying cavity length $L$. The cavity contains a wedged sample 1 $\mathrm{cm}$ in length with wedge angle $\alpha=3 \mathrm{mrad}$. The initial ray is launched on-axis at $r=0$, starting at the left mirror. The first round trip is indicated by the darker line. The total output in each case reflects 100 round-trips. The cavity becomes unstable for $L>2 R$.

Figure 3.11. Ray tracing output of the first Fresnel reflection from the wedged sample surface for a symmetric cavity with $R=20 \mathrm{~cm}$ for varying cavity length $L$. The cavity contains a wedged sample $1 \mathrm{~cm}$ in length with wedge angle $\alpha=3 \mathrm{mrad}$. The initial ray is launched on-axis at $r=0$, starting at the left mirror. The first round-trip (including the first Fresnel reflection) is indicated by the darker line. The total output in each case contains 100 round-trips. The on-axis launch represents the most extreme case in terms of radial expansion of the rays............................................................3.3-82

Figure 4.1. The $\mathrm{KPb}_{2} \mathrm{Cl}_{5}: \mathrm{Dy}^{3+}$ crystals were grown by the Bridgman technique in a two-zone furnace.

Figure 4.2. Coordination of lead in $\mathrm{KPb}_{2} \mathrm{Cl}_{5}$. The $\mathrm{KPb}_{2} \mathrm{Cl}_{5}$ structure has two symmetry independent lead ions, $\mathrm{Pb} 1$ and $\mathrm{Pb} 2$. (a) $\mathrm{Pb} 1$ is 7-fold coordinated. (b) Pb2 is 9-fold coordinated. (after Ref. [14])

Figure 4.3. Phase diagram of the $\mathrm{KCl}-\mathrm{PbCl}_{2}$ system. (after Ref. [59])....

Figure 4.4. Raman phonon spectrum of $\mathrm{KPb}_{2} \mathrm{Cl}_{5}$. The highest feature occurs near $203 \mathrm{~cm}^{-1}$.

Figure 4.5. Mid-IR transmission spectrum through $5 \mathrm{~mm}$ of $\mathrm{KPb}_{2} \mathrm{Cl}_{5}$

Figure 4.6. Absorption cross section of $\mathrm{KPb}_{2} \mathrm{Cl}_{5}: \mathrm{Dy}^{3+}$ at $15 \mathrm{~K}$ (dark line) and at room temperature (light line), showing line broadening with temperature. Energy level values were determined based on peak-to-peak values of the $15 \mathrm{~K}$ spectrum, and appear in Figure 3.1 .

Figure 4.7. Temporal decay of the $\mathrm{W}$ level upon $1.3 \mu \mathrm{m}$ excitation. The decay rate increases as the sample concentration increases, indicative of concentration-dependent cross-relaxation. Notice the decay is largely single exponential for each sample.

Figure 4.8. The overlap of a) two donor ions and b) a donor and an acceptor ion. The relatively large overlap of the donor ion absorption and emission suggests the hopping mechanism is likely to occur. The latter figure (note the log scale) includes phonon assistance via the multiphonon Stokes sideband contributions to the cross sections.

Figure 4.9. The total decay rate for the $\mathrm{W}$ level $(\mathrm{W})$ is plotted against the square of the $\mathrm{Dy}^{3+}$ concentration $\left(\mathrm{N}_{0}{ }^{2}\right)$. Also shown is the fit $\mathrm{W}=$ 
$\mathrm{W}^{0}+k^{c} \mathrm{~N}_{0}^{2}$, where $k^{c}$ is the cross-relaxation coefficient. The slope implies $k^{c}=1.83 \times 10^{-37} \mathrm{~cm}^{6} \mathrm{~s}^{-1}$

Figure 4.10. Emission cross section of $\mathrm{KPb}_{2} \mathrm{Cl}_{5}$ : $\mathrm{Dy}^{3+}$ calculated with $(2.20)$ using Judd-Ofelt derived radiative lifetimes and branching ratios. Each band was calculated separately and combined manually in the figure for comparison.

Figure 4.11. Emission data used to determine branching ratios $\beta_{J}^{\text {rad }}$ and fluorescence ratios ${ }^{P} \phi_{J / K}$ for samples \#1 and \#4. The scales have been adjusted to conserve the total emission rates for each sample. a) Emission spectrum ${ }^{\mathrm{W}} \mathrm{I}(\lambda)$ obtained by direct excitation of the $\mathrm{W}$ level. Note the scale change near $1.3 \mu \mathrm{m}$ and beyond $4 \mu \mathrm{m}$. The transfer of population from the $\mathrm{W}$ level to the $\mathrm{Y}$ level in the higher doped sample (\#4) can be seen by the relative peak heights of the $W \rightarrow Z$ and $Y \rightarrow Z$ transitions. b) Emission spectrum obtained by direct excitation of the $X$ level. The $1.7 \mu \mathrm{m}(X \rightarrow Z)$ feature is not shown. Note the $2.9 \mu \mathrm{m}(\mathrm{Y} \rightarrow \mathrm{Z})$ feature is dramatically reduced compared to that of the $\mathrm{W}$-level-pumped spectrum in a). c) Emission spectrum obtained by direct excitation of the $A$ level. Only the $A \rightarrow Z$ and $W \rightarrow Z$ transitions are shown.

$4.7-100$

Figure 4.12. The $\mathrm{W}, \mathrm{X}$, and $\mathrm{Y}$ level radiative quantum efficiencies are plotted as a function of the $\mathrm{W}$ level cross-relaxation fraction for sample $\# 1$ according to (3.14)-(3.16). The dashed vertical line indicates the value of $\eta_{\mathrm{w}}^{c}$ determined from lifetime measurements according to (3.5).

Figure 4.13. Absorption spectrum for $\mathrm{RbPb}_{2} \mathrm{Cl}_{5}: \mathrm{Dy}^{3+}$ polarized along three mutually perpendicular axes of the biaxial indicatrix.

Figure 4.14. Unpolarized emission cross section of $\mathrm{RbPb}_{2} \mathrm{Cl}_{5}: \mathrm{Dy}^{3+}$. The sample was pumped at $1.3 \mu \mathrm{m}$.

Figure 4.15. Energy-level diagram of $\mathrm{Tb}^{3+}$. The measured lifetimes are indicated on the diagram.

Figure 4.16. $\mathrm{KPb}_{2} \mathrm{Cl}_{5}: \mathrm{Tb}^{3+}$ absorption spectrum. The ground-state level is ${ }^{7} \mathrm{~F}_{6}$. The sample length was $5.9 \mathrm{~mm}$ and the nominal doping was $2.5 \mathrm{~mol} \%$. The $\mathrm{NH}_{4}{ }^{+}$impurity absorption is visible near $7.2 \mu \mathrm{m}$.......4.8-110

Figure 4.17. Calibrated $\mathrm{KPb}_{2} \mathrm{Cl}_{5}: \mathrm{Tb}^{3+}$ emission spectrum. The cutoff near 5.5 $\mu \mathrm{m}$ is due to the cutoff of the InSb detector. The pump level is the ${ }^{7} \mathrm{~F}_{2}$ at $2.0 \mu \mathrm{m}$.

Figure 4.18. Energy-level diagram of $\mathrm{Er}^{3+}$. The thick upward arrow indicates the pumping transition, while the thin downward arrows indicate observed luminescence.

Figure 4.19. $\mathrm{KPb}_{2} \mathrm{Cl}_{5}: \mathrm{Er}^{3+}$ absorption spectrum. (a) $0.4-1.8 \mu \mathrm{m}$ spectra displays identifiable $\mathrm{Er}^{3+}$ features. (b) $2-12-\mu \mathrm{m}$ spectra (transmission) shows the $\mathrm{NH}_{4}{ }^{+}$absorption features at 3 and $7 \mu \mathrm{m}$, and the combination near $2 \mu \mathrm{m}$. 
Figure 4.20. $\mathrm{KPb}_{2} \mathrm{Cl}_{5}: \mathrm{Er}^{3+}$ emission spectrum. The sample was pumped at $0.81 \mu \mathrm{m}$. Emission on the ${ }^{4} \mathrm{I}_{9 / 2} \rightarrow{ }^{4} \mathrm{I}_{11 / 2}$ transition at $4.5 \mu \mathrm{m}$ was not observed, probably due to a small branching ratio.

$4.8-113$

Figure 4.21. $\mathrm{Nd}^{3+}$ absorption spectrum and energy levels. Note the letter designations of the ${ }^{4} \mathrm{~F}_{5 / 2}$ and ${ }^{4} \mathrm{~F}_{3 / 2}$ levels.

Figure 4.22. Emission spectrum obtained by direct excitation of the $\mathrm{S}$ level of $\mathrm{KPb}_{2} \mathrm{Cl}_{5}: \mathrm{Nd}^{3+}$. Emission from both the ${ }^{4} \mathrm{~F}_{3 / 2}$ " $\mathrm{R}$ " and ${ }^{4} \mathrm{~F}_{5 / 2}$ " $\mathrm{S}$ " level is observed. The data infer a fluorescence ratio ${ }_{\phi_{\mathrm{R} / \mathrm{S}}}$ of 75.56.

Figure 4.23. Calibrated emission spectrum of $\mathrm{KPb}_{2} \mathrm{Cl}_{5}: \mathrm{Nd}^{3+}$ near $5 \mu \mathrm{m}$. The sample was pumped at $0.8 \mu \mathrm{m}$. This data represents emission from the ${ }^{4} \mathrm{I}_{15 / 2},{ }^{4} \mathrm{I}_{13 / 2},{ }^{4} \mathrm{I}_{11 / 2}$ levels. The data is truncated due to the InSb detector cutoff near $5.5 \mu \mathrm{m}$ $.4 .8-116$

Figure 4.24. Multiphonon relaxation rate vs. energy gap in $\mathrm{KPb}_{2} \mathrm{Cl}_{5}$ (diamonds). The source of each data point in indicated on the figure. The curves for YAG, YLF, $\mathrm{LaCl}_{3}$, and $\mathrm{LaBr}_{3}$ are shown for comparison. These data are taken from Ref. [40].

Figure 5.1. $\mathrm{Dy}^{3+}$ energy levels and laser schemes. The upper level of the 4.3$\mu \mathrm{m}$ laser can be pumped directly at $1.7 \mu \mathrm{m}$ or indirectly at $1.3 \mu \mathrm{m}$ with a subsequent NR decay. The upper level of the 2.4- $\mu$ m laser is pumped directly at $1.3 \mu \mathrm{m}$

Figure 5.2. Absorption cross section near $1.7 \mu \mathrm{m}$ in $\mathrm{KPb}_{2} \mathrm{Cl}_{5}: \mathrm{Dy}^{3+}{ }_{\text {......................... }}$-1 121

Figure 5.3. Simulated slope-efficiency curve generated from the laser simulation program discussed in Section 2.5

Figure 5.4. Simulated temporal output for $\mathrm{KPb}_{2} \mathrm{Cl}_{5}: \mathrm{Dy}^{3+}$ laser at $2.4 \mu \mathrm{m}$. The pump pulse was modeled as a Gaussian, and the pumping ratio $r$ $=1.6$. The pump and laser signal levels have been arbitrarily adjusted for clarity.

Figure 5.5. Experimental set-up for 1.3- $\mu \mathrm{m}$-pumped laser experiments. ...............5.1-124

Figure 5.6. Emission spectrum and laser output near $2.4 \mu \mathrm{m}$ in sample \#1 ...........5.1-126

Figure 5.7. Temporal waveform of $\mathrm{KPb}_{2} \mathrm{Cl}_{5}: \mathrm{Dy}^{3+}$ laser at $2.4 \mu \mathrm{m}$. The actual pump pulse is also shown. This can be compared to the predicted waveform from Figure 5.4.

Figure 5.8. Slope efficiency (with respect to absorbed pump energy) curves for $\mathrm{KPb}_{2} \mathrm{Cl}_{5}: \mathrm{Dy}^{3+}$ at $2.43 \mu \mathrm{m}$. $\mathrm{T}$ is the total output coupling.

Figure 5.9. "Findlay-Clay" plot of absorbed energy threshold vs. total transmission. A passive loss of $\mathrm{L}=1.2 \%$ is inferred from the data.

Figure 5.10. "Walk-off" (described by the angle $\delta$ ) occurs when the $\mathrm{S}$ and $\mathrm{k}$ vectors are not parallel, as is the case when the incident light does not lie along a principal axis. The case shown is for a uniaxial crystal, and $\mathrm{i}=$ incident, $\mathrm{o}=$ ordinary, $\mathrm{e}=$ extraordinary. The ordinary and extraordinary rays exit parallel to the incident ray due to refraction. See Ref. [] for the details of double refraction in 
anisotropic crystal. The extraordinary ray is polarized in the plane of the page, the ordinary ray out of the page............................5.1-128

Figure 5.11. Uniaxial walk-off angle $\delta$ vs. incident angle $\theta$ for two values of $n_{e} / n_{o}$. The estimated $\mathrm{KPb}_{2} \mathrm{Cl}_{5}$ index ratio is $n_{e} / n_{o}=1.01$.

Figure 5.12. Simulated slope-efficiency curve generated from the laser simulation program discussed in Section 2.5 ................................5.2-130

Figure 5.13. Actual laser data collected for $\mathrm{KPb}_{2} \mathrm{Cl}_{5}: \mathrm{Nd}^{3+}$ at $1.06 \mu \mathrm{m}$................5.2-132

Figure 6.1. $\mathrm{CaGa}_{2} \mathrm{~S}_{4}: \mathrm{Dy}^{3+}$ was grown by the horizontal gradient freeze technique in a two-zone transparent furnace.

Figure 6.2. Transparent two-zone furnace used to grow crystalline $\mathrm{CaGa}_{2} \mathrm{~S}_{4}: \mathrm{Dy}^{3+}$.

$6.1-134$

Figure 6.3. IR transmission of $\mathrm{CaGa}_{2} \mathrm{~S}_{4}$. Sample thickness was $2.5 \mathrm{~mm}$............6.2-135

Figure 6.4. Index of refraction for $\mathrm{CaGa}_{2} \mathrm{~S}_{4}: \mathrm{Dy}^{3+}$ was calculated using (9.23) along two orthogonal polarization axes. The axis associated with the smaller index is labeled the 'fast' axis, the other is the 'slow' axis. The peaks correspond the $\mathrm{Dy}^{3+}$ absorptions. The average $\Delta \mathrm{n}$ is $\sim 0.15$.

Figure 6.5. An oversimplified view of atoms within and between cleavage planes. The atoms in a cleavage plane are coupled together much differently than those between planes, leading to a large index of refraction difference in the two directions.

Figure 6.6. Absorption cross section of $\mathrm{CaGa}_{2} \mathrm{~S}_{4}: \mathrm{Dy}^{3+}$ at $15 \mathrm{~K}$ and $300 \mathrm{~K}$...........6.3-137

Figure 6.7. Polarized absorption spectrum of $\mathrm{CaGa}_{2} \mathrm{~S}_{4}: \mathrm{Dy}^{3+}$. Notice the absorption is nearly 5 times greater along the 'fast' axis near 1.3 $\mu \mathrm{m}$. Only two of the three principal axes of the biaxial indicatrix were accessible to absorption measurements.

Figure 6.8. Experimentally determined energy levels in Dy:YLF (after Ref. 74) $6.3-138$

Figure 6.9. Calibrated emission spectra for $\mathrm{CaGa}_{2} \mathrm{~S}_{4}: \mathrm{Dy}^{3+}$. (a) full spectrum upon W-level pumping. (b) partial spectrum upon $\mathrm{X}$-level pumping $(1.7 \mu \mathrm{m}$ peak not shown).

Figure 6.10. Absorption spectrum showing the IR-cutoff of $\mathrm{CaGa}_{2} \mathrm{Se}_{4}$. The features at $13.5,15$, and $17.5 \mu \mathrm{m}$ are unassigned.

Figure 6.11. Absorption spectrum of $\mathrm{CaGa}_{2} \mathrm{Se}_{4}: \mathrm{Dy}^{3+}$. The spectrum is similar to $\mathrm{CaGa}_{2} \mathrm{~S}_{4}: \mathrm{Dy}^{3+}$ with the small red-shifted peaks and a nonhypersensitive $1.3 \mu \mathrm{m}$ peak.

Figure 6.12. (a) Calibrated emission spectrum of $\mathrm{CaGa}_{2} \mathrm{Se}_{4}: \mathrm{Dy}^{3+}$ upon $1.3 \mu \mathrm{m}$ pumping. Note the scale changes indicated on the figure. The output is limited to $5.5 \mu \mathrm{m}$ due to the cutoff of the InSb detector. (b) uncalibrated emission spectrum upon $0.9 \mu \mathrm{m}$ pumping. Note the emission from the A level. The larger red-shifted peaks are due to transitions to the Stark level lying $\sim 400 \mathrm{~cm}^{-1}$ above the ground state. 
Figure 6.13. IR transmission spectrum of GeGaAsS glass. Unassigned impurity absorption features near 4.0, 4.9, 6.6, and $9.0 \mu \mathrm{m}$ are observed.

$6.7-146$

Figure 6.14. Absorption cross section for GeGaAsS:Dy ${ }^{3+}$ glass. ........................6.7-146

Figure 6.15. Calibrated emission spectrum of GaGeAsS:Dy ${ }^{3+}$ under (a) Wlevel pumping and (b) X-level pumping......................................6.7-148

Figure 7.1. $\mathrm{Dy}^{3+}$ energy levels and lasing schemes for $\mathrm{CaGa}_{2} \mathrm{~S}_{4}: \mathrm{Dy}^{3+} \ldots \ldots \ldots \ldots \ldots \ldots . .6 .8-150$

Figure 7.2. Predicted thresholds and slope efficiencies for $4.3 \mu \mathrm{m}$ laser action in $\mathrm{CaGa}_{2} \mathrm{~S}_{4}: \mathrm{Dy}^{3+}$. $.7 .1-152$

Figure 7.3. The author is pictured here with the $\mathrm{CaGa}_{2} \mathrm{~S}_{4}: \mathrm{Dy}^{3+}$ laser cavity in the foreground and the Cobra 1725 pump laser in the background. The laser crystal is colored green due to a He-Ne alignment laser.....7.1-154

Figure 7.4. Slope-efficiency curves for $\mathrm{CaGa}_{2} \mathrm{~S}_{4}: \mathrm{Dy}^{3+}$ at $4.3 \mu \mathrm{m}$. T is the total mirror transmission (HR + OC).

Figure 7.5. Findlay-Clay analysis of the $\mathrm{CaGa}_{2} \mathrm{~S}_{4}: \mathrm{Dy}^{3+} 4.3-\mu \mathrm{m}$ laser according to Section 2.6. The data imply a round-trip passive loss of $\mathrm{L}=$ $0.9 \% \pm 0.3 \%$.

Figure 7.6. Emission spectrum and laser output near $4.3 \mu \mathrm{m}$ in $\mathrm{CaGa}_{2} \mathrm{~S}_{4}: \mathrm{Dy}^{3+}$. When purged with nitrogen, the laser operates at the peak (4.31 $\mu \mathrm{m})$ of the fluorescence spectrum. When unpurged, the added loss due to ambient $\mathrm{CO}_{2}$ absorption shifts the laser to a longer wavelength $(4.38 \mu \mathrm{m})$.

$7.1-157$

Figure 7.7. Predicted slope-efficiency curves for $\mathrm{CaGa}_{2} \mathrm{~S}_{4}: \mathrm{Dy}^{3+}$ at $2.4 \mu \mathrm{m}$.........7.2-159

Figure 7.8. Slope-efficiency curves for $\mathrm{CaGa}_{2} \mathrm{~S}_{4}: \mathrm{Dy}^{3+} 2.4-\mu \mathrm{m}$ laser. ...................7.2-160

Figure 7.9. Findlay-Clay plot for the $\mathrm{CaGa}_{2} \mathrm{~S}_{4}: \mathrm{Dy}^{3+}$ laser at $2.4 \mu \mathrm{m}$. The data imply a passive loss of $3.9 \%$.

Figure 7.10. $\mathrm{CaGa}_{2} \mathrm{~S}_{4}: \mathrm{Dy}^{3+} 2.4-\mu \mathrm{m}$ emission showing laser signal and emission cross section. $7.2-161$

Figure 7.11. Stark-level structure (not to scale) of the $W$ and $Z$ (ground) manifolds of $\mathrm{CaGa}_{2} \mathrm{~S}_{4}: \mathrm{Dy}^{3+}$, showing the 1.4- $\mu \mathrm{m}$ laser transition. The exact Stark levels participating in the transition are not known.

Figure 7.12. Predicted slope-efficiency curves for the $\mathrm{CaGa}_{2} \mathrm{~S}_{4}: \mathrm{Dy}^{3+}$ laser at $1.4 \mu \mathrm{m}$. $7.3-164$

Figure 7.13. Measured slope-efficiency curve for the $\mathrm{CaGa}_{2} \mathrm{~S}_{4}: \mathrm{Dy}^{3+}$ laser at $1.4 \mu \mathrm{m}$. The total output coupling was $2.2 \%$. $.7 .3-165$

Figure 7.14. The laser emission is centered at $\sim 1.397 \mu \mathrm{m}$ with a FWHM of $\sim 1.5 \mathrm{~nm}$..... . $7.3-166$

Figure 7.15. Absorption and emission cross section near $1.4 \mu \mathrm{m}$. The laser signal at $1.397 \mu \mathrm{m}$ is also shown for comparison. The McCumber-derived emission spectrum and the measured emission spectrum are in reasonably good agreement.

Figure 9.1. Index of refraction $n$ as a function of total transmission $T$. (a) The sample transmission $T_{s}=1$ and multiple reflections are included. 
(b) Same as the previous case, but with $T_{s}=0.98$. (c) $T_{s}=1$ and multiple reflections are not included.

Figure 9.2. Cut-off frequency vs. maximum phonon frequency $h v_{\max }$ for the materials listed in Table 9.8. The slope of the line indicates cutoff occurs with a two-phonon absorption.

Figure 9.3. Simulated input waveform, normalized so that the maximum signal is unity. Signals with low $T / \tau$ don't have time to charge to their maximum level.....

Figure 9.4. $V^{*}$ represents the ratio of the displayed lock-in signal to the maximum signal for a given $T / \tau$ ratio, where $T$ is the chopping period (50\% duty cycle) and $\tau$ is the signal lifetime.

Figure 9.5. A plot of the transmitted power $P$ past a razor blade at position $x$ in a Gaussian TEM $_{00}$ beam. The spot size $w$ is obtained from the fit to $(9.30)$ 


\section{List of Tables}

Table 1.1. Room-temperature, solid-state laser transitions above 2 microns

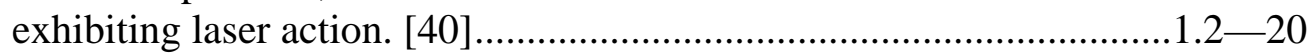

Table 3.1. Transmission, threshold, and slope efficiency data for Nd:YAG

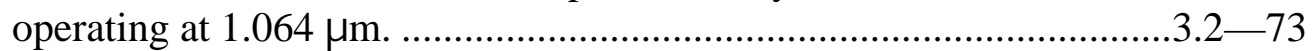

Table 4.1. The $\mathrm{Dy}^{3+}$ concentration of the five samples used in our study. The concentration of sample \#1 was measured directly, and that of samples \#2-\#5 were determined by comparative absorption. ................4.3-90

Table 4.2. Results of the Judd-Ofelt analysis for $\mathrm{KPb}_{2} \mathrm{Cl}_{5}: \mathrm{Dy}^{3+}$.

Table 4.3. Measured radiative branching ratios according to (3.10). 4.7-101

Table 4.4. Experimental values for the fluorescence ratios as defined in (3.11)(3.13). The superscript refers to the pumping level, while the subscripts refer to the originating levels of the emissions.

Table 4.5. Radiative, multiphonon, and cross-relaxation quantum efficiencies as determined by the analysis of Section 3.1. Radiative lifetimes were determined as the ratio of the fluorescence lifetime $\left(\tau^{\text {meas }}\right)$ to the radiative quantum efficiency $\left(\eta^{\mathrm{rad}}\right)$...

Table 4.6. Total branching ratios as defined in (3.4).

Table 4.7. Measured data from Table 4.3 and Table 4.5 compared to the JuddOfelt data from Table 4.2. Average values were used for the measured data whenever applicable.

Table 5.1. Values used for the threshold and slope estimates for the $\mathrm{KPb}_{2} \mathrm{Cl}_{5}: \mathrm{Dy}^{3+}$ laser at $2.4 \mu \mathrm{m}$ according to $(2.81)$ and $(2.88)$.............5.1-122

Table 5.2. Measured threshold and slope efficiency for the $\mathrm{KPb}_{2} \mathrm{Cl}_{5}: \mathrm{Dy}^{3+}$ laser at $2.4 \mu \mathrm{m}$

Table 5.3. Values used for the threshold and slope estimates for the $\mathrm{KPb}_{2} \mathrm{Cl}_{5}: \mathrm{Nd}^{3+}$ laser at $1.06 \mu \mathrm{m}$ according to (2.81) and (2.88)...........5.2-130

Table 6.1. Results of the Judd-Ofelt analysis for $\mathrm{CaGa}_{2} \mathrm{~S}_{4}: \mathrm{Dy}^{3+}$. $6.4-139$

Table 6.2. Measured branching ratios, radiative lifetimes, and radiative quantum efficiencies for $\mathrm{CaGa}_{2} \mathrm{~S}_{4}: \mathrm{Dy}^{3+}$ are compared to JuddOfelt derived data from Table 6.1. The measured total lifetimes and peak emission cross sections (calculated via (2.20) ) are also indicated.

Table 6.3. Results of the Judd-Ofelt analysis for GeGaAsS:Dy ${ }^{3+}$.

Table 6.4. Measured branching ratios, radiative lifetimes, and radiative quantum efficiencies for GeGaAsS:Dy ${ }^{3+}$ are compared to JuddOfelt derived data from Table 6.3. The measured total lifetimes and peak emission cross sections (calculated via (2.20) ) are also indicated.

Table 7.1. Values used for the threshold and slope estimates for the $\mathrm{CaGa}_{2} \mathrm{~S}_{4}: \mathrm{Dy}^{3+}$ laser at $4.3 \mu \mathrm{m}$ according to $(2.81)$ and $(2.88)$............ 7.1-152

Table 7.2. Summary of $\mathrm{CaGa}_{2} \mathrm{~S}_{4}: \mathrm{Dy}^{3+} 4.31 \mu \mathrm{m}$ laser threshold and slope data....7.1-156 Table 7.3. Values used for the threshold and slope estimates for the $\mathrm{CaGa}_{2} \mathrm{~S}_{4}: \mathrm{Dy}^{3+}$ laser at $2.4 \mu \mathrm{m}$ according to (2.81) and (2.88). $7.2-158$ 
Table 7.4. Summary of $\mathrm{CaGa}_{2} \mathrm{~S}_{4}: \mathrm{Dy}^{3+}$ laser results

Table 7.5. Values used for the threshold and slope estimates for the $\mathrm{CaGa}_{2} \mathrm{~S}_{4}: \mathrm{Dy}^{3+}$ laser at $1.4 \mu \mathrm{m}$ according to (2.81) and (2.88).

Table 8.1. Radiative quantum efficiencies for $\mathrm{Dy}^{3+}$ energy levels in some of the hosts studied in this report.

Table 9.1. Magnetic dipole line strength matrix for various transitions in $\mathrm{Pr}^{3+}$. The coupling coefficients are taken from the energy eigenvectors in Ref. []. $S^{\mathrm{MD}}$ values are given in units of $10^{-20} \mathrm{~cm}^{2}$.

Table 9.2. Magnetic dipole line strength matrix for various transitions in $\mathrm{Er}^{3+}$. The coupling coefficients are taken from the energy eigenvectors in Ref. [79]. $\mathrm{S}^{\mathrm{MD}}$ values are given in units of $10^{-20} \mathrm{~cm}^{2}$...

Table 9.3. Magnetic dipole line strength matrix for various transitions in $\mathrm{Dy}^{3+}$. The coupling coefficients are taken from the energy eigenvectors in Ref. []. $\mathrm{S}^{\mathrm{MD}}$ values are given in units of $10^{-20} \mathrm{~cm}^{2}$.

Table 9.4. Magnetic dipole line strength matrix for various transitions in $\mathrm{Tm}^{3+}$. The coupling coefficients are taken from the energy eigenvectors in Ref. []. $\mathrm{S}^{\mathrm{MD}}$ values are given in units of $10^{-20}$ $\mathrm{cm}^{2}$.

Table 9.5. Magnetic dipole line strength matrix for various transitions in $\mathrm{Nd}^{3+}$. The coupling coefficients are taken from the energy eigenvectors in Ref. []. $\mathrm{S}^{\mathrm{MD}}$ values are given in units of $10^{-20} \mathrm{~cm}^{2}$.

Table 9.6. Magnetic dipole line strength matrix for various transitions in $\mathrm{Ho}^{3+}$. The coupling coefficients are taken from the energy eigenvectors in Ref. []. $S^{\mathrm{MD}}$ values are given in units of $10^{-20} \mathrm{~cm}^{2}$.

Table 9.7. Magnetic dipole line strength matrix for various transitions in $\mathrm{Tb}^{3+}$. The coupling coefficients are taken from the energy eigenvectors in Ref. []. $\mathrm{S}^{\mathrm{MD}}$ values are given in units of $10^{-20} \mathrm{~cm}^{2}$.

Table 9.8. Cut-off and maximum phonon frequencies for well-known laser materials. The cut-off frequency, measured here, was taken as that which corresponded to $10 \%$ transmission. Maximum phonon frequency references are indicated. 


\section{Introduction}

\subsection{Motivation and Scope}

Solid-state lasers are devices in which the active gain medium is comprised of a solidstate material. While this material in general can be an insulator (dielectric) or semiconductor, the focus of this research is concerned with dielectrics. The most common dielectric lasers consist of rare-earth ions incorporated substitutionally into crystalline or glassy hosts. Single crystal or glass hosts are needed due to the requirement of very low scattering losses in gain-providing devices. Rare-earth ions are attractive because they have strong absorption bands at wavelengths where pump sources are readily available, and because the induced transitions are relatively insensitive to the particular host. Lasers operating at wavelengths in the visible and near IR (500 $\mathrm{nm}<\lambda<2000 \mathrm{~nm}$ ) have been widely studied because hosts that support such wavelengths (without suffering from nonradiative decay) are readily available. Nonradiative decay typically occurs when the phonon energy of the host material is on the order of the energy gap of the transition involved. Thus as we move to longer wavelengths for our laser devices (and thus to smaller energy gaps), we must find dielectric hosts with smaller phonon energies.

Solid-state host materials with low maximum phonon energies and high luminescence quantum yields have been the subject of a great deal of research for applications in $1.3-\mu \mathrm{m}$ telecommunications amplifiers, IR quantum counters, IR phosphors, and long-wavelength $(4-10 \mu \mathrm{m})$ lasers. ${ }^{1,2,3,4,5,6} \quad$ Indeed, 4.8 and $4.9 \mu \mathrm{m}$ emissions have recently been observed in $\mathrm{Tb}^{3+}$ and $\mathrm{Ho}^{3+}$ doped gallium lanthanum sulfide (GLS) glass, respectively. ${ }^{7,8}$ High quantum efficiency of these devices requires low 
phonon energy hosts to avoid luminescence quenching. A 'rule-of-thumb' indicates that to achieve acceptable quantum efficiency for emission from a given energy level, the energy gap to the next lowest level must span at least 4-5 (maximal energy) phonons. Sulfide and chloride hosts have received the most attention since oxide and fluoride hosts typically have maximum phonon energies in excess of $500 \mathrm{~cm}^{-1}$ (rendering them inefficient above $4 \mu \mathrm{m}$ ). Currently, optical parametric oscillator systems (OPO's) are typically used to cover wavelengths beyond $4 \mu \mathrm{m}$. They incorporate both non-linear media and pump sources, leading to some complexity and sensitivity in the optical system. These challenges could be mitigated if direct solid-state mid-IR lasers were found. The longest wavelength reported to date in a room-temperature, solid-state host medium is $7.2 \mu \mathrm{m}$ in $\mathrm{Pr}^{3+}$-doped $\mathrm{LaCl}_{3},{ }^{9}$ a low-phonon-energy chloride host that is notoriously moisture-sensitive. Identifying a low-phonon-energy crystal that incorporates rare-earth ions and resists attack by moisture has been difficult. We have identified $\mathrm{CaGa}_{2} \mathrm{~S}_{4}$ and $\mathrm{KPb}_{2} \mathrm{Cl}_{5}$ as promising new hosts for mid-IR applications. ${ }^{10,11,12,13}$ Previous studies of $\mathrm{KPb}_{2} \mathrm{Cl}_{5}$ were concerned with the luminescence mechanism of divalent lead emission centers important for visualization of UV-radiation, ${ }^{14}$ while those for $\mathrm{CaGa}_{2} \mathrm{~S}_{4}$ were concerned with phosphor studies. These hosts are not hygroscopic, incorporate rareearth ions, and have low maximum-phonon frequencies $\left(350 \mathrm{~cm}^{-1}\right.$ and $200 \mathrm{~cm}^{-1}$ for $\mathrm{CaGa}_{2} \mathrm{~S}_{4}$ and $\mathrm{KPb}_{2} \mathrm{Cl}_{5}$, respectively). Direct determination of the quantum efficiencies, decay rates, emission and absorption cross sections, and other optical properties is important in the evaluation of these hosts as candidates for applications requiring mid-IR operation. Since these hosts were not recognized for their mid-IR laser potential, little was reported about them is this regard. Indeed, growth of large bulk samples had not 
previously been attempted for $\mathrm{CaGa}_{2} \mathrm{~S}_{4}$, and while some efforts were made to grow bulk $\mathrm{KPb}_{2} \mathrm{Cl}_{5}$, no attempt was made at rare-earth doping. The growth and characterization of these two novel hosts constitutes the majority of this thesis. As these hosts were only discovered in the course of our investigations, we also present results for other hosts studied during early phases of the research. Laser results for $\mathrm{CaGa}_{2} \mathrm{~S}_{4}: \mathrm{Dy}^{3+}$ and $\mathrm{KPb}_{2} \mathrm{Cl}_{5}: \mathrm{Dy}^{3+}$ and $\mathrm{KPb}_{2} \mathrm{Cl}_{5}: \mathrm{Nd}^{3+}$ are also presented. Besides the Summary in Chapter 8 , the Appendices in Chapter 9, and the References in Chapter 10, the thesis contains seven chapters as follows.

The remainder of Chapter 1 includes a review of the fundamentals of lowphonon-frequency hosts and rare-earth ions. The underlying reasons for the need for hosts with low-phonon frequencies are discussed in Section 1.2. Section 1.3 discusses rare-earth ions and why they are so prevalent and important in solid-state laser devices.

In Chapter 2 the historical development of all the basic physics involved in the characterization of solid-state lasers and hosts is presented. Section 2.1 discusses the important concept of the emission cross section as well as methods developed for obtaining its value. Multiphonon decay, which is highly relevant to low-phononfrequency hosts, is developed in Section 2.2. Another process that is more prevalent in low-phonon-frequency hosts is that of nonradiative energy transfer. Since fluorescence lifetimes are typically much longer in low-phonon-frequency hosts, processes like nonradiative energy transfer have more of a chance to occur. These issues are discussed in Section 2.3. Section 2.4 discusses the important technique developed by Judd ${ }^{18}$ and Ofelt ${ }^{19}$ in which radiative rate and branching ratio data can be determined from measured absorption cross section data. Laser output modeling for pulsed laser operation is 
discussed in Section 2.5, where coupled-cavity rate equations are developed that can be solved by standard numerical analysis techniques for the temporal laser output. Laser threshold and extraction efficiency can also be determined from this analysis. Finally, Section 2.6 develops laser threshold and slope efficiency formulas developed for steadystate applications but useful in predicting laser dynamics. Also discussed in this section are the so-called "Caird" and "Findlay-Clay" plots, analyses that can be performed with the laser output data to calculate such things as passive loss and intrinsic slope efficiency.

Chapter 3 develops some ideas that were motivated by the unique aspects of working with low-phonon energy hosts and mid-IR lasers. Section 3.1 develops a method to experimentally measure radiative quantum efficiencies, branching ratios, and multiphonon decay rates for low-phonon-frequency hosts. This method is based on measured emission spectra from the energy levels of concern. Section 3.2 discusses a novel analysis technique to determine the emission cross section from laser threshold and slope data. A key feature of this technique is that it is independent of the passive losses inside the resonator. Data for $\mathrm{Nd}^{3+}$-doped YAG will be given as an example of the application of the technique. Section 3.3 discusses the consequence of a wedge (i.e. nonparallel surfaces) in a high-refractive-index laser sample on the laser performance.

Chapter 4 is concerned with the growth and characterization of $\mathrm{KPb}_{2} \mathrm{Cl}_{5}: \mathrm{Dy}^{3+}$. Sample preparation, crystal properties, absorption and emission spectra, Judd-Ofelt analysis, and quantum efficiency measurements are all presented.

Laser results obtained from $\mathrm{KPb}_{2} \mathrm{Cl}_{5}$ samples are given in Chapter 5. These include predictions, experimental set-ups, and laser data and analysis for $\mathrm{KPb}_{2} \mathrm{Cl}_{5}: \mathrm{Dy}^{3+}$ at $2.4 \mu \mathrm{m}, \mathrm{KPb}_{2} \mathrm{Cl}_{5}: \mathrm{Nd}^{3+}$ at $1.06 \mu \mathrm{m}$, and $\mathrm{KPb}_{2} \mathrm{Cl}_{5}: \mathrm{Tb}^{3+}$ at $5.5 \mu \mathrm{m}$. 
Chapters 6 and 7 are similar to Chapters 4 and 5 but with $\mathrm{CaGa}_{2} \mathrm{~S}_{4}$ samples. Laser data is presented for $\mathrm{CaGa}_{2} \mathrm{~S}_{4}: \mathrm{Dy}^{3+}$ at $1.4,2.4$, and $4.3 \mu \mathrm{m}$.

\subsection{Low-Phonon-Frequency Hosts}

In general, a material must possess certain properties to be considered suitable as a laser host. First, it must be able to transmit the desired laser wavelengths. Second, it must be able to incorporate impurity ions (i.e. rare-earth ions) into its lattice. Third, it must have chemical properties (such as toxicity, hardness, and stability) sufficient for use in commercial applications. Finally, the host must be able to support radiative emission (i.e. resist nonradiative quenching) at the desired laser wavelength.

To extend solid-state lasing capabilities into the mid-IR region (beyond $\sim 4 \mu \mathrm{m}$ ) at room temperature, new hosts are needed because traditional oxide and fluoride hosts are unable to support radiative emission at these wavelengths. This luminescence quenching typically arises from nonradiative decay whereby the energy of an excitation is absorbed in the host by one or more lattice phonons. Indeed, it is the ability to suppress nonradiative processes in favor of radiative processes that indicates a host's usefulness as a mid-IR laser material. A useful figure of merit for a transition between an upper energy level $E_{2}$ and a lower level $E_{1}$ is the radiative quantum efficiency, defined by $\eta^{\text {rad }} \equiv$ $A /(A+W)=(1+W / A)^{-1}$, where $A$ and $W$ characterize the rate of radiative and nonradiative decay between the levels, respectively. In the ideal case where all the decay is radiative, $\eta^{\text {rad }}=1$. Conversely, the worst case has $\eta^{\text {rad }}=0$. The wavelength $\lambda$ of the radiative transition is given by $\lambda=h c / \Delta E$, where $h$ is Planck's constant, $c$ is the speed of light, and $\Delta E=E_{2}-E_{1}$. The radiative quantum efficiency of the transition depends strongly on the host in which the energy levels reside. As we shall see later in the text, as the energy gap 
$\Delta E$ decreases (corresponding to an increase in wavelength $\lambda$ ), the nonradiative rate $W$ increases (approximately) exponentially, while the radiative rate $A$ decreases as $\Delta E^{3}$. Thus, for a given host, as we move to longer wavelengths (e.g. farther into the mid-IR), $\eta^{\text {rad }}$ suffers both from a decreasing radiative rate $A$ and an increasing nonradiative rate W. It turns out that the host environment has little effect on the radiative rate $A$ but a large effect on $W$. Choosing a suitable host thus consists of identifying a material that minimizes the nonradiative rate $W$ (while still maintaining the necessary qualities listed at the beginning of this section).

While the theory of nonradiative decay will be developed in a later section, we have seen that nonradiative decay is mediated through lattice phonons. Multiphonon decay has been shown to be the dominant process characterizing $W$. Since multiphonon decay is a higher-order process, it is believed that the interaction involves the highestenergy phonons of the lattice, as this would minimize the order of the process. Thus minimizing the nonradiative rate $W$ in a laser material can be accomplished by minimizing the highest-energy vibrational feature of the material's fundamental phonon spectrum. In general terms, the vibrational energy of an interaction is given by $\bar{v}=\sqrt{k / \mu}$, where $k$ is an effective force constant and $\mu$ is the reduced mass of the vibrating constituents. While the force constant plays an important role in the binding process, the highest-energy feature will generally occur between the lightest constituents of the host. Minimizing the highest-energy feature will require that these (lightest) constituents be relatively large. In short, hosts with larger constituents will typically have smaller maximum phonon energies. 
Traditional hosts are either oxide based, such as $\mathrm{Y}_{3} \mathrm{Al}_{5} \mathrm{O}_{12}$ (YAG), or fluoride based, such as $\mathrm{YLiF}_{4}(\mathrm{YLF})$. Oxides typically have favorable mechanical and thermal properties due to the strong nature of the ionic bonds. A list of mid-IR, room-temperature, solid-state lasers appears in Table 1.1, indicating the paucity of lasers above $\sim 4 \mu \mathrm{m}$.

Table 1.1. Room-temperature, solid-state laser transitions above 2 microns exhibiting laser action. [40]

\begin{tabular}{|c|c|c|c|}
\hline Dopant & Transition & Host & $\begin{array}{c}\text { Wavelength } \\
(\boldsymbol{\mu} \mathbf{m})\end{array}$ \\
\hline \hline $\mathrm{Er}^{3+}$ & ${ }^{4} \mathrm{~F}_{9 / 2} \rightarrow{ }^{4} \mathrm{I}_{11 / 2}$ & YAG & 2.0 \\
\hline $\mathrm{Tm}^{3+}$ & ${ }^{3} \mathrm{H}_{4} \rightarrow{ }^{3} \mathrm{H}_{6}$ & YAG & 2.2 \\
\hline $\mathrm{Ho}^{3+}$ & ${ }^{5} \mathrm{I}_{7} \rightarrow{ }^{5} \mathrm{I}_{8}$ & YAG & 2.2 \\
\hline $\mathrm{Tm}^{3+}$ & ${ }^{3} \mathrm{~F}_{4} \rightarrow{ }^{3} \mathrm{H}_{5}$ & YAlO & 2.4 \\
\hline $\mathrm{Er}^{3+}$ & ${ }^{4} \mathrm{I}_{11 / 2} \rightarrow{ }^{4} \mathrm{I}_{13 / 2}$ & YAG & 2.9 \\
\hline $\mathrm{Ho}^{3+}$ & ${ }^{5} \mathrm{I}_{6} \rightarrow{ }^{5} \mathrm{I}_{7}$ & YAG & 3.0 \\
\hline $\mathrm{Dy}^{3+}$ & ${ }^{6} \mathrm{H}_{13 / 2} \rightarrow{ }^{6} \mathrm{H}_{15 / 2}$ & $\mathrm{LaF}$ & 3.0 \\
\hline $\mathrm{Ho}_{3}{ }^{3+}$ & ${ }^{5} \mathrm{~S}_{2} \rightarrow{ }^{5} \mathrm{~F}_{5}$ & $\mathrm{YLF}$ & 3.7 \\
\hline $\mathrm{Ho}^{3+}$ & ${ }^{5} \mathrm{I}_{5} \rightarrow{ }^{5} \mathrm{I}_{6}$ & $\mathrm{YLF}$ & 3.9 \\
\hline $\mathrm{Dy}^{3+}$ & ${ }^{6} \mathrm{H}_{11 / 2} \rightarrow{ }^{6} \mathrm{H}_{13 / 2}$ & $\mathrm{YLF}$ & 4.3 \\
\hline $\mathrm{Pr}^{3+}$ & ${ }^{3} \mathrm{~F}_{3} \rightarrow{ }^{3} \mathrm{~F}_{2}$ & $\mathrm{LaCl}$ & 7.2 \\
\hline
\end{tabular}

The logical approach in identifying hosts with lower maximum phonon energies is to study sulfide and chloride hosts, as these elements lie directly under oxygen and fluorine in the Periodic Table, respectively, indicating that they are chemically similar but more massive than their traditional counterparts. It follows that selenide and bromide hosts would also be logical to study. In general we have found that it is very difficult to find a selenide or bromide host that maintains the desired chemical properties of a laser host. This study examines many sulfide and chloride hosts (both glassy and crystalline), as well as some selenide and bromide materials. They include $\mathrm{GeGaS}$ glass, $\mathrm{LaCl}_{3}$, $\mathrm{KPb}_{2} \mathrm{Cl}_{5}, \mathrm{RbPb}_{2} \mathrm{Cl}_{5}, \mathrm{CaGa}_{2} \mathrm{~S}_{4}, \mathrm{CaGa}_{2} \mathrm{Se}_{4}, \mathrm{GaLaS}$ glass. Laser action was demonstrated in two of these, $\mathrm{KPb}_{2} \mathrm{Cl}_{5}$ and $\mathrm{CaGa}_{2} \mathrm{~S}_{4}$. 
Both crystalline and glassy materials can serve well as laser hosts. Physically, the main difference is in the structure of the constituent atoms. By definition, glasses have a random structure and crystals have a regular structure. These physical and chemical differences greatly affect the luminescent properties of the dopant ions incorporated within the host. The random nature of the glassy structure produces a different electric field at the site of each dopant ion, and therefore each ion has a different set of Stark levels. In general, this results in a broadening of the absorption and emission bands and reduced cross sections. From an engineering point of view, glasses can typically be more easily fabricated and more easily drawn into fibers. Thus the choice between crystal and glass ultimately depends upon the nature of the application.

\subsection{Rare-Earths}

Rare-earth ions are important in the development of laser hosts since the electrons involved in optical phenomena are relatively insensitive to the crystal field of the surrounding host material. Of particular interest are the lanthanide ions, which are typically most stable in the triply ionized state. They are characterized by a closed xenon shell ([Kr] $\left.4 d^{10} 5 s^{2} 5 p^{6}\right)$ and the sequential filling of the $4 f$ shell (with the exception of Gadolinium (atomic number $\mathrm{Z}=64$ ) which has an extra $5 d$ electron). Thus $\mathrm{Ce}^{3+}(\mathrm{Z}=58)$ has one $4 f$ electron $\left(4 d^{10} 4 f^{1} 5 s^{2} 5 p^{6}\right)$, while $\mathrm{Yb}^{3+}(\mathrm{Z}=70)$ has $134 f$ electrons $\left(4 d^{10} 4 f\right.$ ${ }^{13} 5 s^{2} 5 p^{6}$ ). The energy levels below $\sim 20,000 \mathrm{~cm}^{-1}$ of the (lanthanide) rare-earths ${ }^{15}$ are shown in Figure 1.1.

Since the $4 f$ shell is not completely filled (with 14 electrons), many $4 f$ levels remain unoccupied. The optical transitions typical of rare-earth spectra are attributed to 
intra- $4 f$ "forced" electric-dipole transitions of electrons already in the $4 f$ shell to these unoccupied $4 f$ levels.

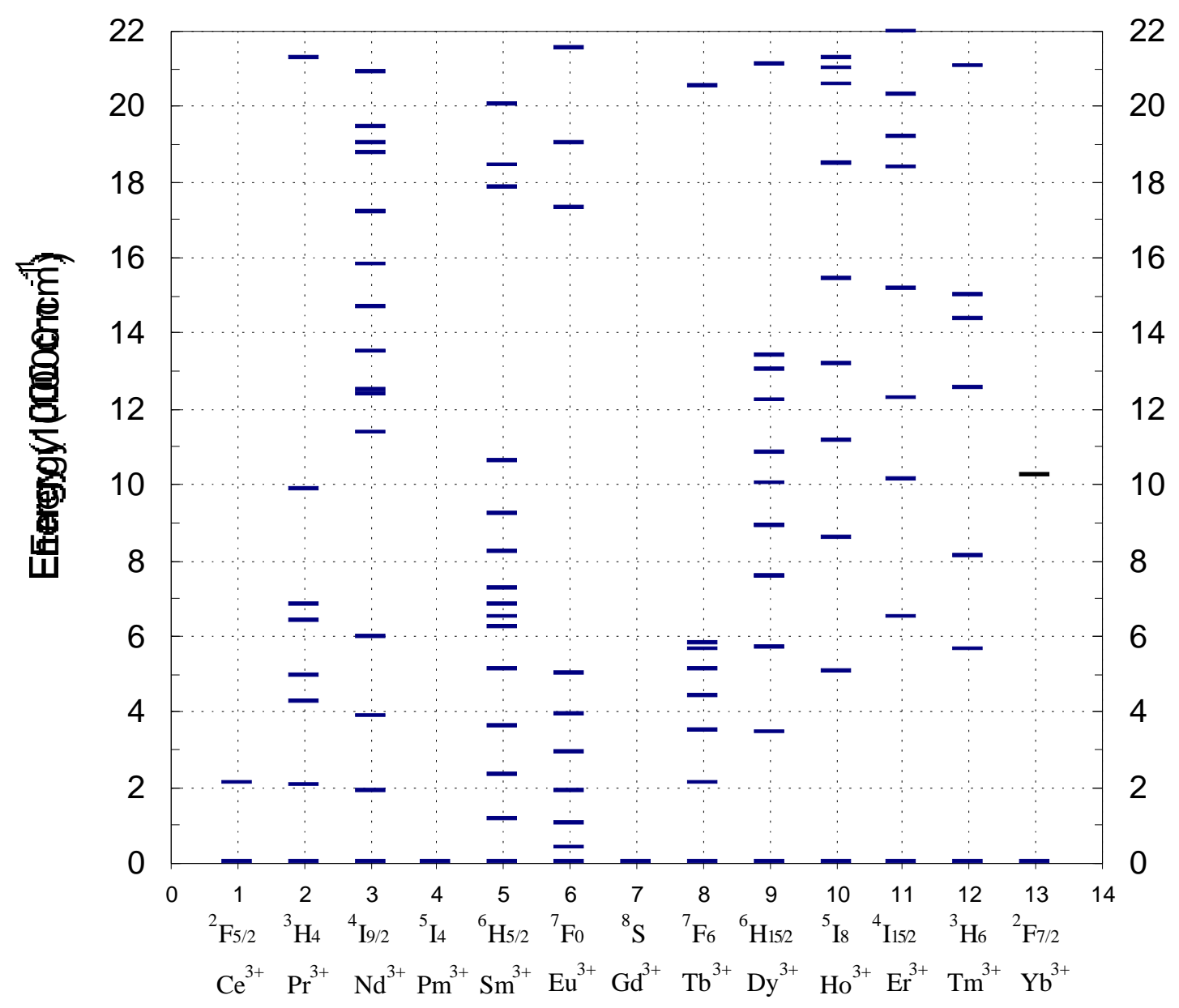

number of $4 f$ electrons

Figure 1.1. Trivalent rare-earth energy levels below $22,000 \mathrm{~cm}^{-1}$. Russell-Saunders groundstate labels are indicated. (data taken from Ref. 15)

The insensitivity to host is a result of the 'lanthanide contraction', which is a consequence of imperfect screening by the $4 f$ electrons that leads to an increase in effective nuclear charge as the atomic number increases in the lanthanide series. ${ }^{16,17}$ Thus the $4 f$ wavefunctions actually lie within the closed $5 s^{2} 5 p^{6}$ xenon shell for the lanthanide rare-earths, and the $4 f$ electrons are shielded from the surrounding 
environment by the $5 s$ and $5 p$ electrons. This leads to energy levels that have small host-induced splitting and are only weakly mixed with higher energy states. This results is a greatly reduced vibronic interaction with the host, which leads to weak nonradiative relaxation of excited states (and thus longer excited-state lifetimes), sharp spectral features, large cross sections and high quantum yields.

For a free ion, electric-dipole transitions between $4 f$ states are strictly parity forbidden. The optical spectra in rare-earths are therefore thought to be caused by forced electric dipole, magnetic-dipole, or electric-quadrupole transitions. The transition probabilities of the latter two transitions are typically at least an order of magnitude less than those of forced electric dipole transitions, although magnetic-dipole transitions can sometimes be comparable. Forced electric dipole transitions arise from noncentrosymmetric interactions of the ions with the potential $V$ of the surrounding crystal field. The Hamiltonian for an ion placed in a crystal field can be expressed as

$$
H=H_{\text {free }}+V \text {, }
$$

where $H_{\text {free }}=H_{C F}+H_{c o l}+H_{s o}$ is the Hamiltonian of the free ion with known eigenvalues and eigenfunctions possessing complete spherical symmetry. $H_{C F}$ is the central field Hamiltonian, in which each electron is assumed to move independently in a spherically symmetric potential formed by the average potential of the electrons and the nucleus. The spherical symmetry enables the angular component to be expressed as hydrogenic spherical harmonics. Complete solutions of the central field problem can be constructed from hydrogenic states. Accordingly, $L$ and $S$ are "good" quantum numbers, and $4 f$ states are usually described in terms of Russell-Saunders $L-S$ coupling, where $L$ and $S$ are added vectorally to form the total angular momentum $J$, and the states are labeled ${ }^{2 S+1} L_{J}$. In the 
central field approximation, all these states are degenerate. $H_{c o l}$ is the energy of the electrostatic Coulomb interaction between the outer $4 f$ electrons. This interaction lifts the angular degeneracy and produces states which depend on $L$ and $S$ but not $J . H_{s o}$ is spinorbit Hamiltonian. This interaction lifts the degeneracy in $J$, splitting the $L S$ states into $J$ levels. Intermediate coupling must be used to diagonalize the spin-orbit and Coulomb interaction simultaneously. This leads to eigenstates which are linear combinations of different $L S$ states, and therefore eigenstates of $J$ but not $L$ or $S$. These states are traditionally still labeled by their Russell-Saunders identification, using the dominant $L S$ contribution. Figure 1.2 indicates the effect of each of these interactions on the lower levels of $\operatorname{Pr}^{3+}$ ion.

Due to the lanthanide contraction discussed above, the potential provided by the crystalline environment $V$ in (1.1) is relatively small and can be treated as a perturbation. It can be expanded in terms of the operator $\mathbf{C}_{q}^{(k)}$ as

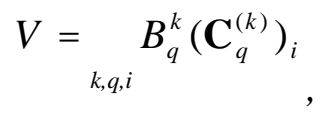

where $\mathbf{C}_{q}^{(k)}$ are related to the spherical harmonics as $\mathbf{C}_{q}^{(k)}=[4 \pi /(2 k+1)]^{1 / 2} Y_{k q}, B_{q}^{k}$ are the coefficients of the expansion, and the summation $i$ is carried out over all the $4 f$ electrons of the ions, for which only the terms with $k \leq 6$ are non-vanishing. The even- $k$ terms in 


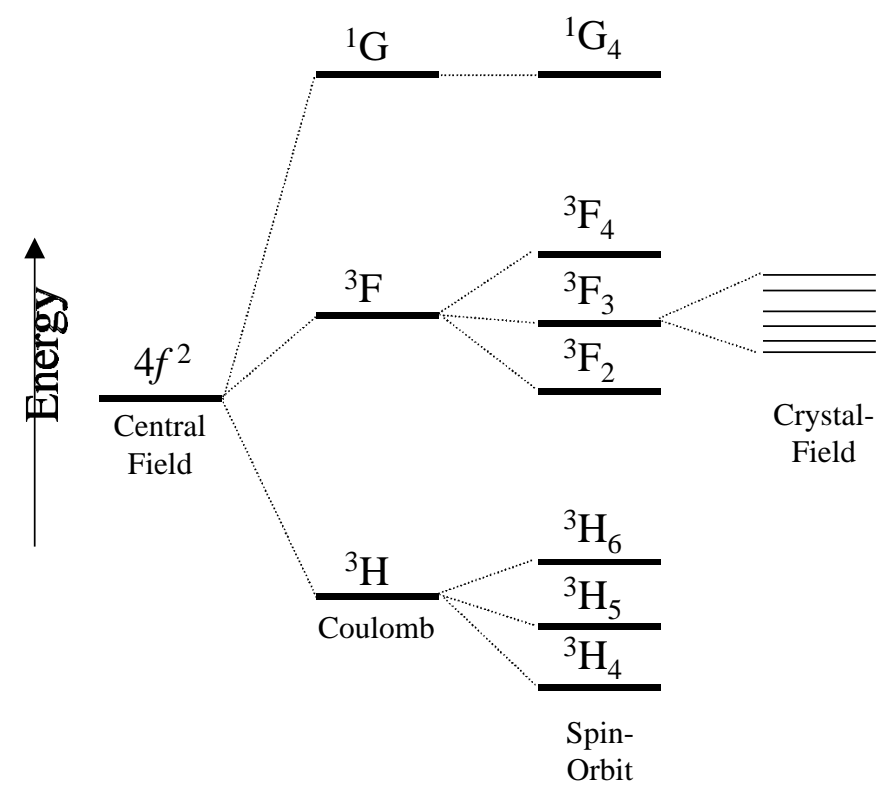

Figure 1.2. Partial energy level diagram (not to scale) indicating the effect of the different interaction Hamiltonians on $\mathrm{Pr}^{3+}$. The spin-orbit interaction gives rise to the Russell-Saunders levels indicated in Figure 1.1. The crystal-field Hamiltonian splits these levels into Stark levels. (Taken from Ref. [30])

the expansion split the free-ion J multiplets into Stark components and thus determine the wavelengths at which absorption or emission occur. The odd- $k$ terms admix higher lying states of opposite parity, such as $4 f^{n-1} 5 d$ or $4 f^{n-1} 5 g$, and thus determine the absorption or emission intensity since electric-dipole transitions can only occur to these oppositeparity states.

To determine the strength of such a forced electric-dipole transition we need to evaluate the matrix element $\left\langle A|\mathbf{P}| A^{\prime}\right\rangle$, where $\mathbf{P}$ is the electric-dipole operator. The mixed-parity states $\langle A|$ and $\left|A^{\prime}\right\rangle$ can be represented (to first order) as linear combinations of the wavefunctions $\left|4 f^{n}[S L] J\right\rangle$ of the ground $4 f^{n}$ configurations with 
the wavefunctions $|\varepsilon\rangle$ of excited states of the opposite parity in the presence of the perturbing potential $V^{\text {odd }}$ as described above

$$
\begin{gathered}
\langle A|=\left\langle 4 f^{n}[S L] J J_{z}\right|+\frac{\left\langle 4 f^{n}[S L] J J_{z}\left|V^{\text {odd }}\right| \varepsilon\right\rangle\langle\varepsilon|}{E\left(4 f^{n}[S L] J J_{z}^{\prime}\right)-E(\varepsilon)}, \\
\left|A^{\prime}\right\rangle=\left|4 f^{n}\left[S^{\prime} L^{\prime}\right] J^{\prime} J_{z}^{\prime}\right\rangle+{ }_{\varepsilon} \frac{|\varepsilon\rangle\left\langle\varepsilon\left|V^{\text {odd }}\right| 4 f^{n}\left[S^{\prime} L^{\prime}\right] J^{\prime} J_{z}^{\prime}\right\rangle}{E\left(4 f^{n}\left[S^{\prime} L^{\prime}\right] J^{\prime} J_{z}^{\prime}\right)-E(\varepsilon)} .
\end{gathered}
$$

Since the symmetry in a crystal is lower than spherical, $\mathbf{P}$ can be resolved into its spherical components as

$$
\mathbf{P}=e_{\rho, i} r_{i}\left(\mathbf{C}_{\rho}^{(1)}\right)_{i} \ldots \underset{\rho}{\quad} \mathbf{P}_{\rho}^{(1)},
$$

where $\rho=0$ gives the $z$-component corresponding to absorption or emission of $\pi$ polarized light, and $\rho= \pm 1$ gives the $x \pm i y$ components for $\sigma$-polarized light. The strength of a polarized transition between the parity-mixed states $\langle A|$ and $\left|A^{\prime}\right\rangle$ will depend on the non-vanishing matrix elements $\left\langle A\left|\mathbf{P}_{\rho}^{(1)}\right| A^{\prime}\right\rangle$ given by

$$
\begin{aligned}
\left\langle A\left|\mathbf{P}_{\rho}^{(1)}\right| A^{\prime}\right\rangle= & \frac{\left\langle 4 f^{n}[S L] J J_{z}\left|V^{\text {odd }}\right| \varepsilon\right\rangle\left\langle\varepsilon\left|\mathbf{P}_{\rho}^{(1)}\right| 4 f^{n}\left[S^{\prime} L^{\prime}\right] J^{\prime} J_{z}^{\prime}\right\rangle}{E\left(4 f^{n}[S L] J J_{z}\right)-E(\varepsilon)} \\
+ & \frac{\left\langle 4 f^{n}[S L] J J_{z}\left|\mathbf{P}_{\rho}^{(1)}\right| \varepsilon\right\rangle\left\langle\varepsilon\left|V^{\text {odd }}\right| 4 f^{n}\left[S^{\prime} L^{\prime}\right] J^{\prime} J_{z}^{\prime}\right\rangle}{E\left(4 f^{n}\left[S^{\prime} L^{\prime}\right] J^{\prime} J_{z}^{\prime}\right)-E(\varepsilon)} .
\end{aligned}
$$

where the summation is over all excited states $|\varepsilon\rangle$. The solution to (1.6) is difficult since in general the excited-state wavefunctions $|\varepsilon\rangle$ and energy levels $E(\varepsilon)$ and the odd portion of the crystal field $V^{\text {odd }}$ are not known. Simplifying assumptions made by $\mathrm{Judd}^{18}$ and 
Ofelt ${ }^{19}$ allowed these matrix elements to be determined. These assumptions, along with the results that follow, will be the topic of Section 2.4 in the next chapter. 


\section{Theoretical Background}

\subsection{Emission Cross Sections}

Emission cross sections are important in the prediction and analysis of laser performance. The cross section of a transition has units of area and is an indicator of the strength of the transition. It is analogous to the absorption cross section which represents the effective interaction area of an ion as "seen" through the eyes of an incoming photon. The interaction area is that which if encountered by the photon, a transition will take place, otherwise the photon will pass unperturbed. A macroscopic analogy would cast the target ion as the "eight ball" and the incoming photon as the "cue ball". The cross section of the "eight ball" as seen by the "cue ball" would then simply be the cross-sectional area of the "eight ball". Note the cross section of the ion could be larger than is physical area since the effect of the dipole fields can be felt outside its physical boundaries.

In this section we develop theoretical methods for determining the emission cross section $\sigma^{e m}$. The two equations we will develop are the $\mathrm{F} \Omega$ chtbauer-Ladenburg relation and the McCumber ${ }^{20}$ relation. The former extracts the cross section from basic absorption and emission relations, while the latter obtains the emission cross section from knowledge about the absorption cross section using the principle of reciprocity. Both of these methods were used to calculate emission cross section data for use in laser performance models.

In the development of the $F \llbracket$ chtbauer-Ladenburg relation, it is useful to first derive the Einstein $A$ and $B$ coefficients. These arise when considering optical absorption and emission in a material. The cross section will emerge when we relate the radiation 
density $u$ of a radiation field passing through a dielectric medium to the energy flux $I$ in the medium via $I=\mathrm{c} \cdot u / n$, where $\mathrm{c}$ is the speed of light and $n$ is the refractive index. We assume the transition is due to a resonance with energy levels $E_{1}$ and $E_{2}$, where $E_{1}$ is the ground state, $E_{2}$ is an excited state, and $\Delta E=E_{2}-E_{1}=h v$, see Figure 2.1.

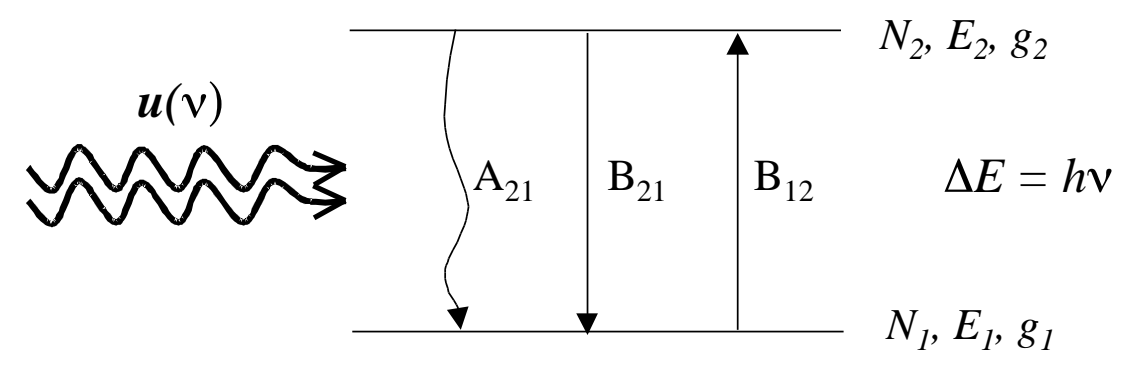

Figure 2.1. Illustration of concepts used to determine the emission cross section. The symbols are defined in the text.

If a quasi-monochromatic electromagnetic wave of nominal frequency $v$ passes through the material, then the population of the ground state will be depleted at a rate proportional to the ground-state population $N_{1}$ and the incident radiation density $u(v)$ [units of $\mathrm{J} \cdot \mathrm{s} / \mathrm{cm}^{3}$ ]

$$
\frac{f N_{1}}{f t}=-B_{12} u(v) N_{1},
$$

where $B_{12}$ is the constant of proportionality for the upward transition (with dimensions $\left.\mathrm{cm}^{3} /(\mathrm{s} \cdot \mathrm{J})\right)$. Similarly, an excited atom in $E_{2}$ with population $N_{2}$ can be 'stimulated' to make the downward transition

$$
\frac{f N_{2}}{f t}=-B_{21} u(v) N_{2} \text {. }
$$

An excited atom in $E_{2}$ can also decay spontaneously according to 


$$
\frac{f N_{2}}{f t}=-A_{21} N_{2}
$$

where $A_{21}$ is the constant of proportionality for spontaneous emission (with dimensions s' $\left.{ }^{1}\right)$. Assuming the total population is conserved, we may write

$$
\frac{f N_{1}}{f t}=-\frac{f N_{2}}{f t}=B_{21} u(v) N_{2}-B_{12} u(v) N_{1}+A_{21} N_{2} .
$$

In thermal equilibrium the time derivatives will be zero, so that we can write

$$
B_{21} u(v) N_{2}+A_{21} N_{2}=B_{12} u(v) N_{1}
$$

Solving for $u(v)$ and using the Boltzmann relation $N_{2} / N_{1}=\left(g_{2} / g_{1}\right) \exp (-h v / k T)$, where $g_{\mathrm{i}}$ is the degeneracy of the $i^{\text {th }}$ level, we obtain

$$
u(v)=\frac{\left(A_{21} / B_{21}\right)}{\left(g_{1} B_{12} / g_{2} B_{21}\right) \exp (h v / k T)-1}
$$

By comparing this result to Planck's law for the distribution of radiation density in thermal equilibrium,

$$
u(v)=\frac{8 \pi h v^{3}}{c^{3} \exp (h v / k T)-1}
$$

we find

$$
\frac{A_{21}}{B_{21}}=\frac{8 \pi n^{3} v^{2} h v}{c^{3}} \quad \text { and } \quad B_{21}=\frac{g_{1}}{g_{2}} B_{12}
$$

The stimulated transition rate is then given by 


$$
W_{21}^{\text {stim }}=B_{21} u(v)=\frac{A_{21} c^{3}}{8 \pi n^{3} h v^{3}} u(v) .
$$

To obtain an expression for the emission cross section, we need to consider linewidthbroadened energy levels with a energy separation centered at $v_{0}$ with half-width $\Delta v$. The incident radiation is centered at $v$ with half-width $d v$. In this case the population is described in terms of the normalized linewidth function $g\left(v^{\prime}-v_{0}\right)$, and (2.9) becomes

$$
W_{21}^{\text {stim }}={ }_{-\times}^{+\times} \frac{A_{21} c^{3}}{8 \pi n^{3} h v^{3}} u\left(v^{\prime}\right) g\left(v^{\prime}-v_{0}\right) d v^{\prime}
$$

For a monochromatic field $u\left(v^{\prime}\right)=u_{v} \delta\left(v^{\prime}-v\right)$, which yields

$$
W_{21}^{\text {stim }}=\frac{A_{21} c^{3} u_{v}}{8 \pi n^{3} h v^{3}} g\left(v-v_{0}\right)=\frac{A_{21} c^{2} I_{v}}{8 \pi n^{2} h v^{3}} g\left(v-v_{0}\right)
$$

where $I_{v}=c u_{v} / n$ is the energy flux. The absorption rate follows directly from (2.8)

$$
W_{12}=\frac{g_{2}}{g_{1}} \frac{A_{21} c^{2} I_{v}}{8 \pi n^{2} h v^{3}} g\left(v-v_{0}\right)
$$

When this flux propagates in the medium a distance $d x$, we have

$$
\frac{f I_{v}}{f x}=\left(n_{2} W_{21}-n_{1} W_{12}\right) h v
$$

where $n_{\mathrm{i}}$ are the population densities $n_{\mathrm{i}}=N_{\mathrm{i}} / V$. Using (2.11) and (2.12) this becomes (neglecting the spontaneous emission term)

$$
\frac{f I_{v}}{f x}=n_{2}-\frac{g_{2}}{g_{1}} n_{1} \frac{A_{21} c^{2} g\left(v-v_{0}\right)}{8 \pi n^{2} v^{2}} I_{v}
$$


Defining the term in brackets as the population inversion $\Delta \mathrm{n}$, we find upon integration over the crystal length $l$ that

$$
I_{v}(l)=I_{0} \exp \left[\Delta n \sigma^{e m}(v) l\right],
$$

where

$$
\sigma^{e m}(v)=\frac{A_{21} c^{2}}{8 \pi n^{2} v^{2}} g\left(v-v_{0}\right)
$$

is the emission cross section of the $2 \rightarrow 1$ transition. Note if the interaction can be considered as electric dipole, the spontaneous transition probability $A_{21}$ can be written in terms of the intitial state $\left|E_{2}\right\rangle$ and the final state $\left|E_{1}\right\rangle$ as ${ }^{21}$

$$
A_{21}=\frac{32 \pi^{3}}{3 \hbar c^{3}} v^{3}\left|\left\langle E_{2}|\vec{p}| E_{1}\right\rangle\right|^{2}
$$

where $\vec{p}$ is the electric dipole operator.

Since we often work in the wavelength domain $(\lambda=c / v)$, it is convenient to write the cross section in terms of the measured emission intensity $I(\lambda)$ (arbitrary W/nm). We know that $I(\lambda) d \lambda \propto h v g(v) d v$, so

$$
g(v) \propto \frac{I(\lambda)}{h v} \frac{d \lambda}{d v} \propto \frac{I(\lambda)}{h v} \frac{1}{v^{2}} \propto(\text { const }) \lambda^{3} I(\lambda)
$$

The proportionality constant comes from normalization since $g(v) d v=1$ :

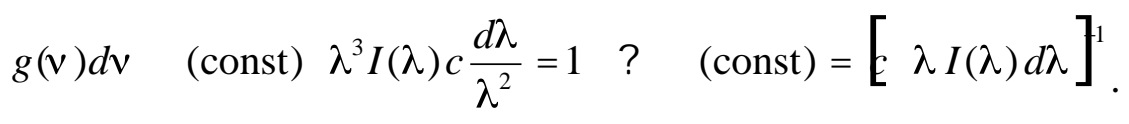


Substituting into (2.16) we obtain the $\mathrm{F} \preccurlyeq$ chtbauer-Ladenburg relation for the emission cross section, expressed in terms of the wavelength $\lambda$ and the measured emission intensity $I(\lambda)$

$$
\sigma^{e m}(\lambda)=\frac{\lambda^{2} \beta_{21}}{8 \pi n^{2} \tau_{2}^{r a d}} \frac{\lambda^{3} I(\lambda)}{c \lambda I(\lambda) d \lambda}
$$

where the integral takes places over the bandwidth of the transition $2 \rightarrow 1$, and we have substituted $A_{21}=\beta_{21} / \tau_{2}^{\text {rad }}$. Thus $\sigma^{e m}$ can be calculated if we know the index of refraction $n$ of the host, radiative branching ratio, radiative lifetime, and emission intensity $I(\lambda)$ of the $2 \rightarrow 1$ transition. An important feature of (2.20) in regards to mid-IR lasers is that the branching ratio necessary to maintain a constant emission cross section varies as $1 / \lambda^{2}$. That is, if we assume the radiative lifetime and effective bandwidth remain constant for transitions of different wavelength (same upper state level, for example), then the cross section will remain constant if the $\lambda^{2} \beta_{21}$ product remains constant. Consequently, the cross section for $\beta_{21}=100 \%$ at $1 \mu \mathrm{m}$ will be the same as $\beta_{21}$ $=1 \%$ at $10 \mu \mathrm{m}$. Figure 2.2 shows a plot of $\beta_{21}$ vs. $\lambda$ where the product $\lambda^{2} \beta_{21}$ is conserved. 


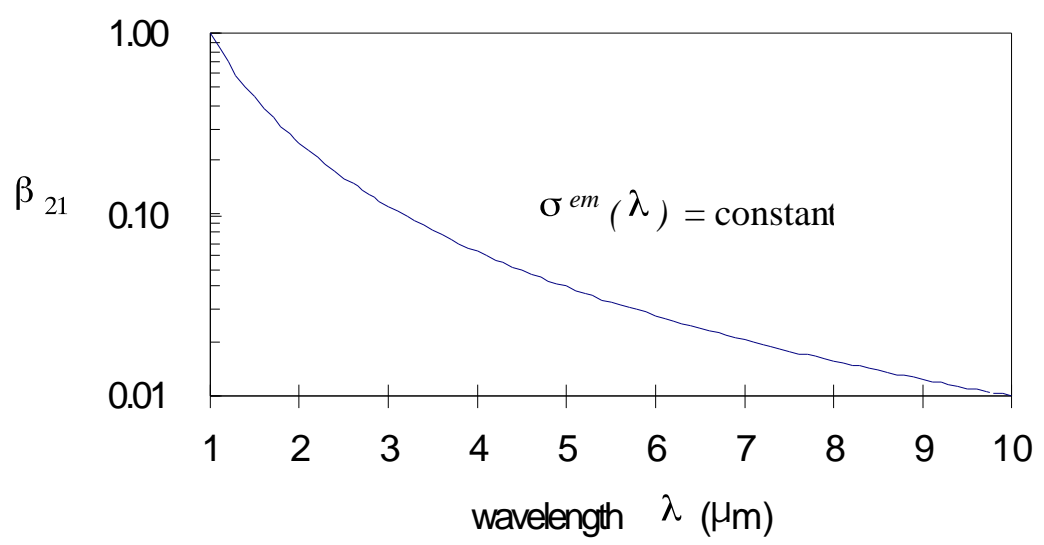

Figure 2.2. Plot showing the relationship between the radiative branching ratio $\beta_{21}$ and the wavelength $\lambda$ that conserves the emission cross section $\sigma^{e m}$, all other factors being equal.

If the crystal is anisotropic, we can write the polarization dependent form of (2.20) as

$$
\sigma_{\alpha}^{e m}(\lambda)=\frac{A_{21} \lambda^{2}}{8 \pi n^{2}} \frac{\lambda^{3} I_{\alpha}(\lambda)}{c\left[\frac{1}{3} \sum_{\beta} I_{\beta}(\lambda)\right] \lambda d \lambda}
$$

where $I_{\alpha}(\lambda)$ is the emission spectrum polarized along the $\alpha=\mathrm{x}, \mathrm{y}$, or $\mathrm{z}$ axis.

The McCumber relation allows us to determine $\sigma^{e m}$ purely from measurements of the absorption cross section $\sigma^{a b s}$. Figure 2.3 illustrates the concepts used in the analysis.

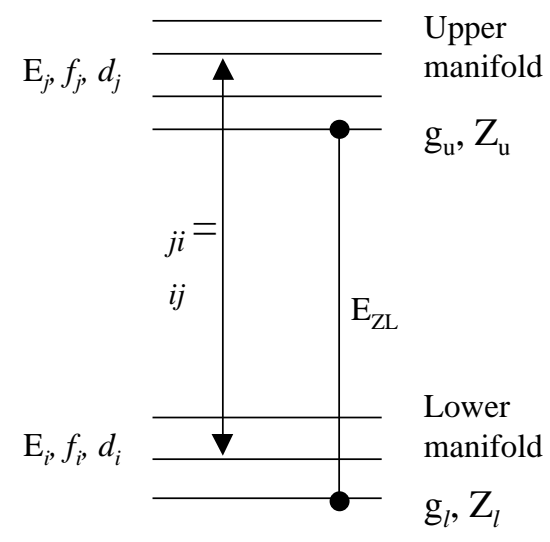

Figure 2.3. Schematic of the concepts used in this section. The upper manifold has a partition function $Z_{u}$, and has $g_{u}$ states associated with it, each with energy $E_{j}$ (measured relative to the lowest level in the manifold) and Boltzmann occupation factor $f_{j}$. Similar statements are true of the lower manifold. 
Following the method decribed by Payne, et.al. ${ }^{22}$, we write the manifold-manifold emission cross section as the degeneracy-weighted sum of the individual cross sections between the Stark levels $j$ and $i$ :

$$
\sigma^{e m}(v)=f_{i, j} \sigma_{j i}(v) d_{i}
$$

where $d_{i}$ is the degeneracy of the $i^{\text {th }}$ Stark level and the Boltzmann occupation fraction $f_{j}$ is given by

$$
f_{j}=\frac{d_{j} \exp \left(-E_{j} / k T\right)}{d_{k} \exp \left(-E_{k} / k T\right)}
$$

where $E_{j}$ is the energy of the $j^{\text {th }}$ Stark level in the upper manilfold, measured relative to the lowest level in the manifold. The demoninator in this expression is recognized as the partition function of the upper manifold, $Z_{u}$. A similar expression can be written for the absorption cross section from the lower manifold:

$$
\sigma^{a b s}(v)=f_{i, j} \sigma_{i j}(v) d_{j}
$$

An example of the role of temperature in the shape of the absorption and emission cross section is shown in Figure 2.4. If we label the "zero-line" energy separation of the lowest Stark levels of the upper and lower manifold as $E_{Z L}$, we can relate the energies $E_{j}$ and $E_{i}$ by

$$
E_{j}-E_{i}=h v-E_{Z L} .
$$




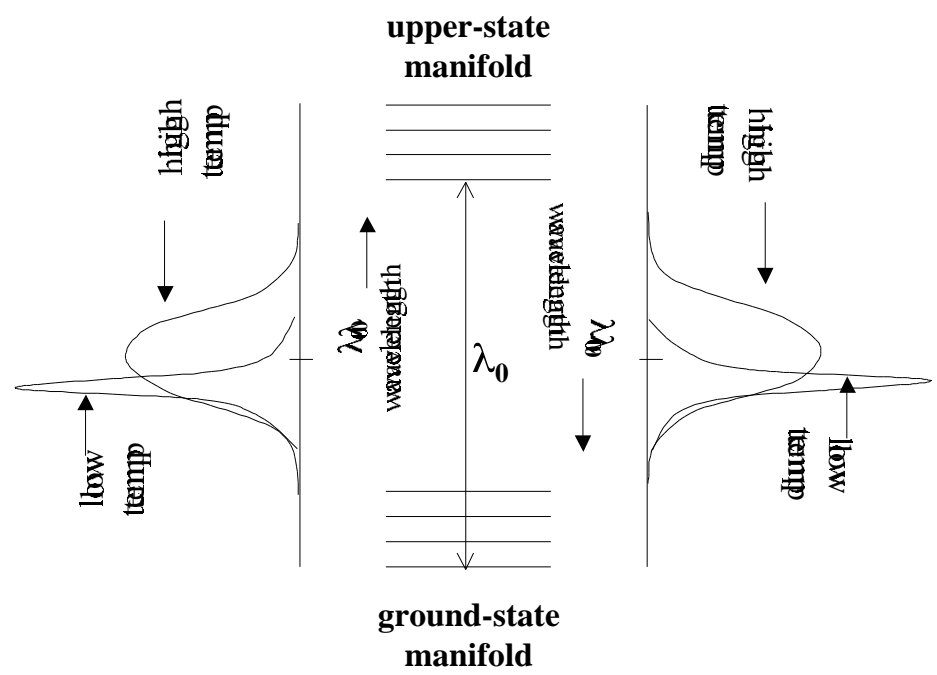

Figure 2.4. Example of an emission and absorption spectrum at different temperatures. At low temperatures (near $0 \mathrm{~K}$ ), only the lowest groundstate Stark level is populated, and absorption transitions will originate only from this level. At higher temperatures, more Stark levels are populated so that more transitions can take place. This broadens the spectrum considerably. The area under the spectra are conserved. A similar process applies to the emission spectrum.

The McCumber relation can be obtained by dividing (2.22) by (2.24), employing (2.25), and utilizing the reciprocity relation $\sigma_{i j}=\sigma_{j i}$ :

$$
\sigma^{e m}(v)=\sigma^{a b s}(v) \frac{Z_{l}}{Z_{u}} \exp \left[E_{Z L} / k T\right] ? \exp [-h v / k T] .
$$

Evaluation of the term in braces requires knowledge of the detailed Stark structure of the manifolds involved in the transition. Accurate vales of $E_{Z L}$ are important due to the exponential nature of the factor involved. In the absence of this detailed knowledge, we can view this term as a constant which is temperature dependent but frequency independent and write

$$
\sigma^{e m}(v)=\sigma^{a b s}(v) \kappa(T) \exp [-h v / k T] .
$$


For rare-earths at room-temperature it is often a good approximation that $\sigma_{i j}$ and $\sigma_{j i}$ are independent of $i$ and $j$ due to averaging over many Stark components. ${ }^{23}$ This is especially true in glassy hosts, where each ion sees a slightly different ligand field. This is, in fact, one of the approximations adopted in the Judd-Ofelt analysis of Section 2.4. In this case it can be shown (see Appendix A) that

$$
g_{u} \quad \sigma^{e m}(v) d v=g_{l} \quad \sigma^{a b s}(v) d v,
$$

where $g_{u, l}=2 J_{u, l}+1$ is the degeneracy of the upper and lower level, respectively. The value of $\kappa(T)$ then becomes

$$
\kappa(T)=\frac{g_{l} \quad \sigma^{a b s}(v) d v}{g_{u} \sigma^{a b s}(v) \exp (-h v / k T) d v} .
$$

Thus equations (2.27) and (2.29) serve as a convenient method of determining the emission cross section if the lower manifold of interest is the ground-state and the absorption cross section is known.

\subsection{Multiphonon Decay}

As we have seen, the nonradiative decay rate $W$ plays an important role in the dynamics of a laser transition. Indeed, in Section 1.2 we saw that minimizing $W$ played a crucial role in choosing a mid-IR host material. At low-modest rare-earth concentrations where ion-ion interactions are negligible, the dominant contribution to $W$ comes from multiphonon decay. In this section we develop a theoretical basis for the experimentally determined exponential dependence of the multiphonon decay rate $W^{M P}$ on the energy gap $\Delta \mathrm{E}$ known as the "energy gap law": 


$$
W^{M P}=A \exp (-\alpha \Delta E)\left[1-\exp \left(-\hbar \omega_{e f f} / k T\right)\right]^{-p}
$$

where $A$ and $\alpha$ are phenomenological constants determined from experiment which depend on the host but are presumed independent of the rare-earth ion, and $p=\Delta E / \hbar \omega_{\text {eff }}$ represents the number of phonons (characterized by an energy $\hbar \omega_{\text {eff }}$ ) emitted in the transition. Note these phonons are considered indistinguishable and therefore follow Bose-Einstein statistics. These concepts are depicted in Figure 2.5 below.

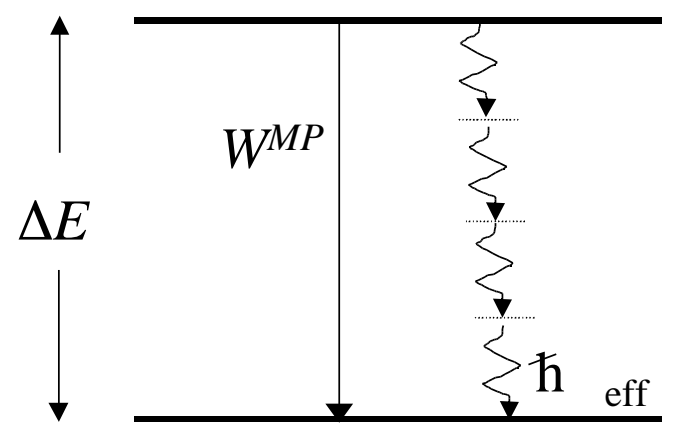

Figure 2.5. multiphonon decay (at temperature $T$ ) is characterized by a decay rate $W^{M P}$, an energy gap $\Delta \mathbf{E}$, and an effective phonon energy $\hbar \omega_{\text {eff }}$.

The orbit-lattice interaction of a rare-earth ion with its dynamical crystalline surroundings is considered to be due to the modulation of the crystalline electric field by the lattice vibrations. If each ion is assumed to behave as a point-charge, this interaction manifests itself as a displacement of the ion from its equilibrium position. The crystal field can then be expanded in a Taylor series about this equilibrium position as

$$
V_{O L}=V_{c}+\quad V_{i} Q_{i}+\frac{1}{2} \quad V_{i j} Q_{i} Q_{j}+\ldots
$$


where $V_{c}$ represents the static crystal field, $Q_{i}$ are the normal-mode coordinates, and the coefficients $V_{i}, V_{i j}$, etc. are given by

$$
V_{i}=f V_{c} / f Q_{i}, \quad V_{i j}=f^{2} V_{c} / f Q_{i} f Q_{j}, \quad \ldots
$$

The emission of $p$ phonons can arise from carrying the first-order term to a $p$-th order perturbation, or from the $p$-th order term in a first-order perturbation. $\mathrm{Kiel}^{24}$ was able to show that the probability of a transition from a state $\left|\psi_{a}\right\rangle$ to a state $\left|\psi_{b}\right\rangle$ due to the sum of these two sources can be written ${ }^{25}$

$$
\begin{aligned}
& W^{(p)}=\left.\underset{\substack{i \cdots j, m_{1} \cdots m_{p-1}}}{ } g\left(\omega_{i}\right) \cdots g\left(\omega_{j}\right) \delta\left(E_{b}+\hbar \omega_{i}+\cdots \hbar \omega_{j}-E_{a}\right)\left\langle n_{i}+1\left|Q_{i}\right| n_{i}\right\rangle\right|^{2} \cdots\left|\left\langle n_{j}+1\left|Q_{j}\right| n_{j}\right\rangle\right|^{2} \\
& \leftrightarrow \frac{\left|\left\langle\psi_{b}\left|V_{i}\right| \psi_{m_{p-1}}\right\rangle\right|^{2} \cdots\left|\left\langle\psi_{m_{1}}\left|V_{j}\right| \psi_{a}\right\rangle\right|^{2}}{\left(E_{m_{p-1}}+\hbar \omega_{j}+\cdots+\hbar \omega_{i-1}-E_{a}\right)^{2} \cdots\left(E_{m_{1}}+\hbar \omega_{j}-E_{a}\right)^{2}}
\end{aligned}
$$

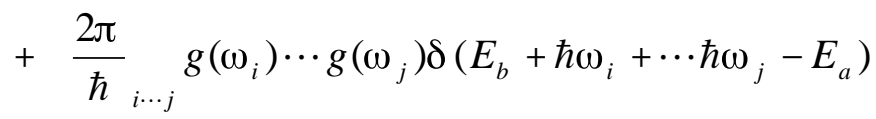

$$
\begin{aligned}
& \leftrightarrow(1 / p !)^{2}\left|\left\langle\psi_{b}\left|V_{i \cdots j}\right| \psi_{a}\right\rangle\right|^{2}\left|\left\langle n_{i}+1\left|Q_{i}\right| n_{i}\right\rangle\right|^{2} \cdots\left|\left\langle n_{j}+1\left|Q_{j}\right| n_{j}\right\rangle\right|^{2}
\end{aligned}
$$

Here, $n_{i} \cdots n_{j}$ are phonon occupation numbers, $\left|\psi_{m_{1}}\right\rangle \cdots\left|\psi_{m_{p-1}}\right\rangle$ are intermediate virtual states, and $g\left(\omega_{i}\right) \cdots g\left(\omega_{j}\right)$ are the densities of phonon states. The complexity of this expression renders it calculationally intractable. However, Layne ${ }^{26}$ was able to show that for weak orbit-lattice coupling, the ratio of the $p$-th order process to the $(p-1)$-th order process is independent of $p$ and can be written:

$$
\varepsilon \ldots \frac{W^{(p)}}{W^{(p-1)}}=\left(\bar{n}_{e f f}+1\right) \varepsilon^{\prime}
$$

where $\varepsilon^{\prime}$ is an ion-lattice coupling constant given by 


$$
\varepsilon^{\prime}=\frac{\hbar(2 m)^{2}}{2 M \omega_{e f f}} ? \frac{\left|\left\langle\psi_{b}\left|V_{o L}\right| \psi_{a}\right\rangle\right|^{2}}{\left(\hbar \omega_{e f f}\right)^{2}}
$$

Here, the effective occupation number $\bar{n}_{\text {eff }}$, the effective phonon frequency $\omega_{\text {eff }}$, and the effective matrix element $\left|\left\langle\psi_{b}\left|V_{O L}\right| \psi_{a}\right\rangle\right|^{2}$ [(dimensions of energy divided normal mode displacement $)^{2}$ ] arise from assuming that the details of the ion-lattice coupling are statistically averaged out for a multiphonon process involving a large number of interacting phonon modes and intermediate states. $M$ is a mass-related coefficient dependent upon the choice of the normal coordinates, and $m$ is the number of modes. The effective phonon frequency $\omega_{\text {eff }}$ is generally taken as that of the highest frequency phonon. $\bar{n}_{\text {eff }}$ is given by the Bose-Einstein average

$$
\bar{n}_{e f f}=\left[\exp \left(\hbar \omega_{e f f} / k T\right)-1\right]^{-1} .
$$

The quantity $\hbar /\left(2 M \omega_{\text {eff }}\right) ?\left(\bar{n}_{\text {eff }}+1\right)$ gives the square of the normal mode displacement. Thus $\varepsilon$ physically represents the square of the energy change caused by the amplitude of the vibration, divided by the square of the energy of the vibration. Because $\varepsilon$ is independent of $p$, we can write $W^{(p)}$ in terms of the zero-th order process $W^{(0)}$ as follows:

$$
W^{(p)}=\frac{W^{(p)}}{W^{(p-1)}} ? \frac{W^{(p-1)}}{W^{(p-2)}} ? \cdots ? \frac{W^{(1)}}{W^{(0)}} ? W^{(0)}=\varepsilon^{p} W^{(0)}=\left(\bar{n}_{e f f}+1\right)^{p} \varepsilon^{\prime p} W^{(0)}
$$

With the definitions

$$
A \ldots W^{(0)} \quad \text { and } \quad \alpha \ldots \frac{\ln \left(1 / \varepsilon^{\prime}\right)}{\hbar \omega_{e f f}}
$$


we recover the exponential "energy-gap law" of (2.30). Notice the dependence of the host appears in the definition of $\alpha$ through the ion-lattice coupling constant $\varepsilon^{\prime}$ and the effective phonon frequency $\omega_{\text {eff }}$. In general, rare-earths have small values of $\varepsilon^{\prime}$, and thus relatively small nonradiative rates, as discussed in Section 1.3. A small coupling constant and a small maximum phonon energy will lead to a small nonradiative rate. A general "rule-of-thumb" is that in order for multiphonon processes not to dominate a transition, its energy gap $\Delta \mathrm{E}$ must be bridged by at least $4-5$ phonons. In a given host, $A$ and $\alpha$ can be determined from the fit (according to $(2.30)$ ) of a few measurements of $W^{M P}$ vs. $\Delta E$ (for energy gaps resulting from any rare-earth ion). Thus if $\hbar \omega_{\text {eff }}$ is known for a given host, we can determine $W^{M P}$ for any gap in any rare-earth ion. Methods for measuring $W^{M P}$ will be discussed in a later section. Examples of such data for other hosts are shown in Figure 2.6.

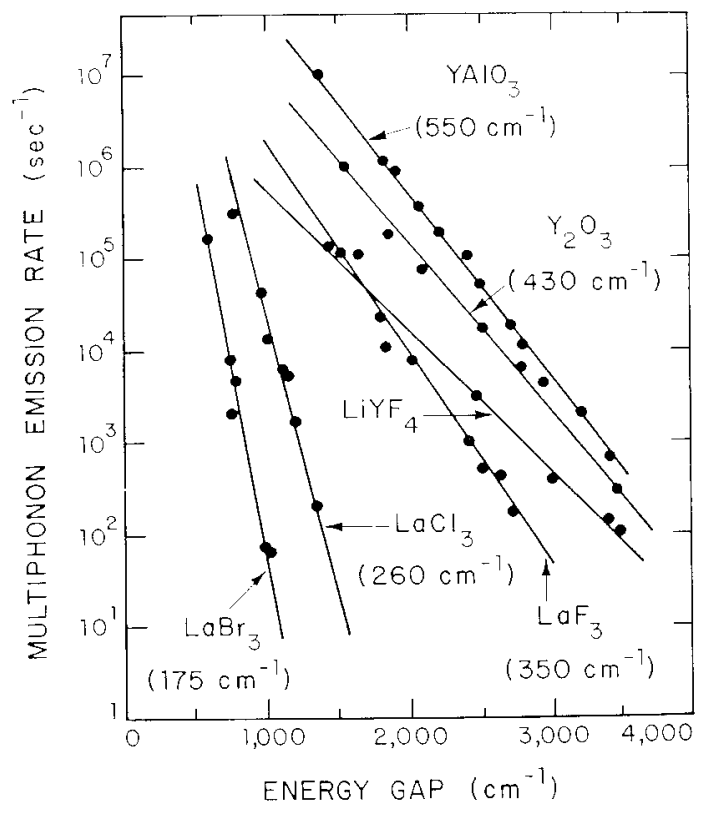

Figure 2.6. Multiphonon emission rate vs. energy gap for various hosts. Effective phonon energies are shown in parentheses. (From ${ }^{27}$ ) 
It is worth noting that the "energy-gap law" was also derived theoretically by Miyakawa and Dexter. ${ }^{28}$ They found results similar to (2.37) and (2.38), but with

$$
\alpha=\frac{\ln (p / g)-1}{\hbar \omega_{e f f}}
$$

where $g$ is an ion-lattice coupling constant which Auzel $^{29}$ reports does not vary greatly from host to host (for typical oxide, fluoride, sulfide, and chloride hosts), with a typical value of $g=0.04-0.05$. Comparing this with (2.38), we conclude

$$
\varepsilon^{\prime}=\frac{e g}{p}
$$

where $e=2.718$. The two results are compatible if it is assumed that $p \approx$ constant $\approx 3$ in (2.40), which is also necessary to make $\alpha$ independent of the energy gap $\Delta \mathrm{E}$. Notice the value of $g=0.05$ makes $\varepsilon^{\prime} \approx 0.05$, confirming the assumption of weak ion-lattice coupling.

\subsection{Nonradiative Energy Transfer}

Nonradiative processes other than multiphonon decay can be important in the decay kinetics of an ion-host system, especially at higher rare-earth concentrations. These processes have been studied extensively in the literature ${ }^{30}$, and this section attempts to summarize the results. The energy transfer process was first studied by Förster ${ }^{31}$ and Dexter $^{32}$ by considering an excitation that is transferred from a donor ion $\mathrm{D}$ to an acceptor ion A, which may be of the same or different species. These neighboring ions are separated by a distance R. The donor ion starts in an excited state $\left|D^{*}\right\rangle$ and the acceptor ion in its ground state $|A\rangle$. The nonradiative interaction $H^{\prime}$ shifts the excitation 
to the acceptor, which ends in an excited state $\left|A^{*}\right\rangle$, leaving the donor in its ground state $|D\rangle$. Figure 2.7 depicts these concepts.
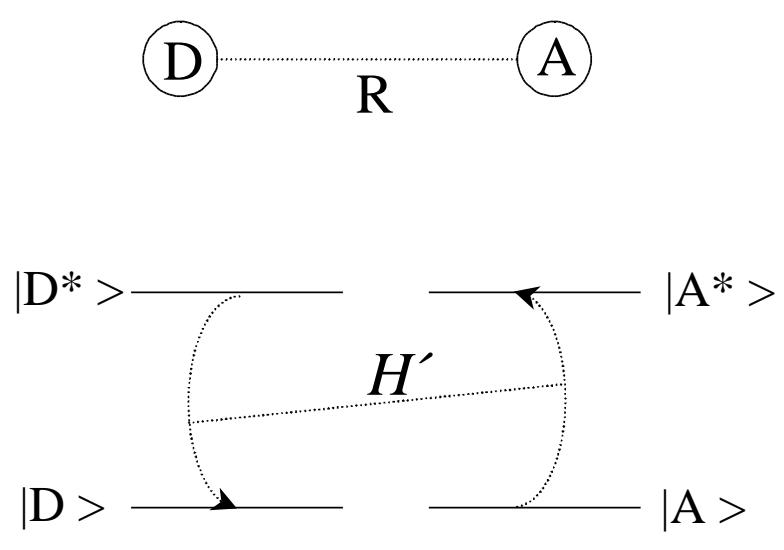

Figure 2.7. Energy is transferred by the nonradiative interaction $H^{\prime}$ from the donor, initially in state $\left|D^{*}\right\rangle$, to the acceptor, initially in state $|A\rangle$. The donor $D$ and acceptor $A$ are separated by a distance $R$. (after Ref. 30)

Assuming the radiative emission transition $\left|D^{*}\right\rangle \rightarrow|D\rangle$ and radiative absorption transition $|A\rangle \rightarrow\left|A^{*}\right\rangle$ have normalized lineshape functions $g^{D}(E)$ and $g^{A}(E)$, respectively, the transition probability is given by Fermi's Golden Rule as

$$
W^{D A}=\frac{2 \pi}{\hbar}\left|\left\langle D, A^{*}\left|H^{\prime}\right| D^{*}, A\right\rangle\right|^{2} \mathrm{~g}^{D}(E) \mathrm{g}^{A}(E) d E
$$

where the overlap integral ensures conservation of energy. The interactions which cause energy transfer are electrostatic coupling, magnetic coupling, and exchange coupling. The electrostatic coupling is the most significant, and varies as $R^{-k}$ where $k=3$ for dipoledipole interactions, $k=4$ for dipole-quadrupole, and $k=5$ for quadrupole-quadrupole interactions. The largest contribution therefore occurs when $H^{\prime}$ is taken as the 
electrostatic dipole-dipole interaction between the electrons on $D$ and on $A$, and probability can be written ${ }^{30}$

$$
W^{D A}=\frac{4 \pi}{3 \hbar n^{4} R^{6}}\left|\left\langle D|\mathbf{P}| D^{*}\right\rangle\right|^{2}|\langle A *|\mathbf{P}| A\rangle|^{2} \quad \mathrm{~g}^{D}(E) \mathrm{g}^{A}(E) d E
$$

where $\mathbf{P}$ is the electric dipole operator and $n$ is the refractive index. By noting

$$
\left|\left\langle D|\mathbf{P}| D^{*}\right\rangle\right|^{2}=\frac{3 n \lambda}{8 \pi^{3}} \sigma^{D}(E),
$$

where $\lambda$ is the transition wavelength and $\sigma^{D}$ is the emission cross section (a similar expression applies for the absorption process), this can be put into the more useful form

$$
W^{D A}=\frac{3 c}{8 \pi^{4} n^{2} R^{6}} \sigma^{D}(\lambda) \sigma^{A}(\lambda) d \lambda .
$$

A critical radius $R_{D A}$ was developed by Förster and Dexter, which is defined as the ionion separation where the nonradiative decay rate $W^{D A}$ is equal to the radiative decay rate $A^{r a d}:$

$$
R_{D A}{ }^{6}=\frac{W^{D A}}{A^{\text {rad }}} R^{6}
$$

A complete picture of nonradiative decay must account for the statistical average of all possible transfers, including transfers between donors (D-D transfer). Indeed, substituting $\mathrm{A} \rightarrow \mathrm{D}$ in (2.44) and (2.45) characterizes the donor-donor transfer rate $W^{D D}$ and the donor-donor critical radius $R_{D D}$, respectively. Huber $^{33}$ started with the set of coupled differential equations 


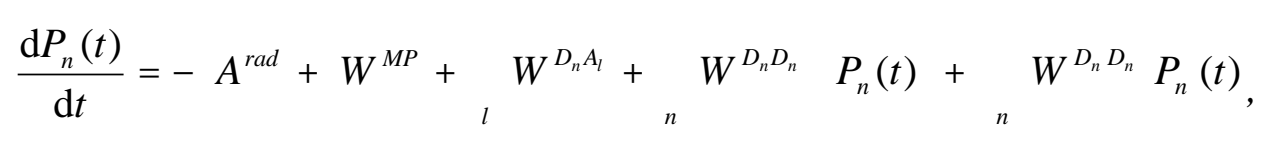

where $W^{M P}$ is the multiphonon emission rate, $P_{n}(t)$ is the probability that the $n$-th donor ion is excited at time $t$, and the intensity $\phi(t)$ of donor luminescence is proportional to $P_{n}(t)$. The total decay rate is assumed to be given by

$$
W=A^{r a d}+W^{M P}+W^{c}=W^{0}+W^{c}
$$

where $W^{0}$ is the radiative and multiphonon component of the decay (which is not concentration-dependent), and $W^{c}$ is the concentration-dependent component of the decay (due to nonradiative energy transfer). Note $W=W^{0}$ in the limit of zero concentration. There are three cases to consider in this general model. First, if there is no energy transfer (i.e. $W^{D A}=W^{D D}=0$ ), we have the trivial case where

$$
\phi_{1}(t)=\exp \left[-W^{0} t\right]
$$

and the decay is purely exponential. Second, considering only dipole-dipole D-A transfers (i.e. $W^{D D}=W^{M P}=0$ ), Förster ${ }^{31}$ was able to write the donor luminescence as

$$
\phi_{2}(t)=\exp \left\lfloor-\gamma t^{1 / 2}\right\rfloor
$$

where

$$
\gamma \ldots \frac{4}{3} \pi^{3 / 2} N_{A} R_{D A}^{3}\left(A^{\text {rad }}\right)^{1 / 2}
$$

Here, $N_{A}$ is the concentration of accpetors. Owing to the $t^{\frac{1}{2}}$ dependence, $\phi_{2}(t)$ displays a nonexponential decay. Third, we consider excitation migration among donors (D-D 
transfer) preceding a dipole-dipole energy transfer between a donor and acceptor. In this case we can identify two situations: (a) when $R_{D A} \gg R_{D D}$ indicating that the direct energy transfer mechanism between a donor and acceptor is much stronger than that between the donors, and (b) when $R_{D D} \gg R_{D A}$ indicating that the donor-donor mechanism is much stronger. The first case is referred to as the diffusion model, ${ }^{34}$ where

$$
\phi_{3}(t)=\exp -\gamma t^{1 / 2} \frac{1+10.87 y+15.5 y^{2}}{1+8.743 y} v^{3 / 4},
$$

where $\gamma$ is given by (2.50) and

$$
y=\frac{1}{2}-\frac{4}{3} \pi N_{D} \sqrt{ }{ }^{4 / 3} R_{D D}{ }^{6} R_{D A}{ }^{-2}\left(A^{r a d} t\right)^{1 / 2},
$$

and $N_{D}$ is the concentration of donors. Asymptotically, the decay rate can be approximated by $\phi_{3}(t)=\exp \left(-W^{c} t\right)$, where

$$
W^{c} \cup[21] R_{D A}^{3 / 2} R_{D D}^{9 / 2} N_{A} N_{D} A^{r a d},
$$

The second case is referred to as the hopping model, ${ }^{35}$ and in this case

$$
\phi_{4}(t)=\exp \left(-W^{c} t\right)
$$

where

$$
W^{c}=\left[\pi(2 \pi / 3)^{5 / 2}\right] R_{D A}{ }^{3} R_{D D}^{3} N_{A} N_{D} A^{r a d} .
$$

The exponential decay is due to the fact that the migration process averages out the random nature of the donor-acceptor distance $R$, so that the donors appear to behave collectively and decay with a common rate. This situation typically arises for rare-earth 
ions where the absorption-emission Stokes shifts are relatively small, giving rise to relatively large D-D overlaps and thus a large value of $R_{D D}$. Because the D-A overlaps are "accidental", the $R_{D A}$ values are typically much smaller.

Figure 2.8 shows the donor decay rate for each of the cases listed above. Curve (a) is purely exponential with decay rate $A^{\text {rad }}$. The nonexponential influence of D-A and D-D transfer can be seen in curves (b) and (d), respectively, and curve (d) shows the decay returning to exponential when the effect of donor migration is large. Because of the their random distribution, some acceptors will be in very close proximity to donor ions, while others will find themselves much more distant. The initial fast decay shown in Figure 2.8(b) is due to the decay of the nearby acceptors. The more distant acceptors will take longer to decay, and are limited by the rate $A^{\text {rad }}$ of an isolated acceptor. The total decay rate $\Phi(t)$ is considered to be a combination of all these processes, and can be written

$$
\ddot{O}(t)=\exp \left\lfloor W^{0} t-\gamma t^{\frac{1}{2}}-W^{c} t\right\rfloor
$$

The above analysis assumes resonant energy transfer. Some transfer processes, however, are not resonant and require phonon assistance. The assistance of phonons in the D-A energy-transfer model can be incorporated by using the exponential relation developed by $\mathrm{Auzel}^{36}$ to include the multiphonon sidebands of the emission and absorption cross sections. The Stokes sideband cross section is given by ${ }^{37}$

$$
\sigma^{\text {Stokes }}(\Delta \bar{E})=\sigma^{\text {elec }}\left(E_{o}\right) \exp \left(-\alpha_{S} \Delta \bar{E}\right)\left[1-\exp \left(-\hbar \omega_{\text {eff }} / k T\right)\right]^{-\bar{N}}
$$




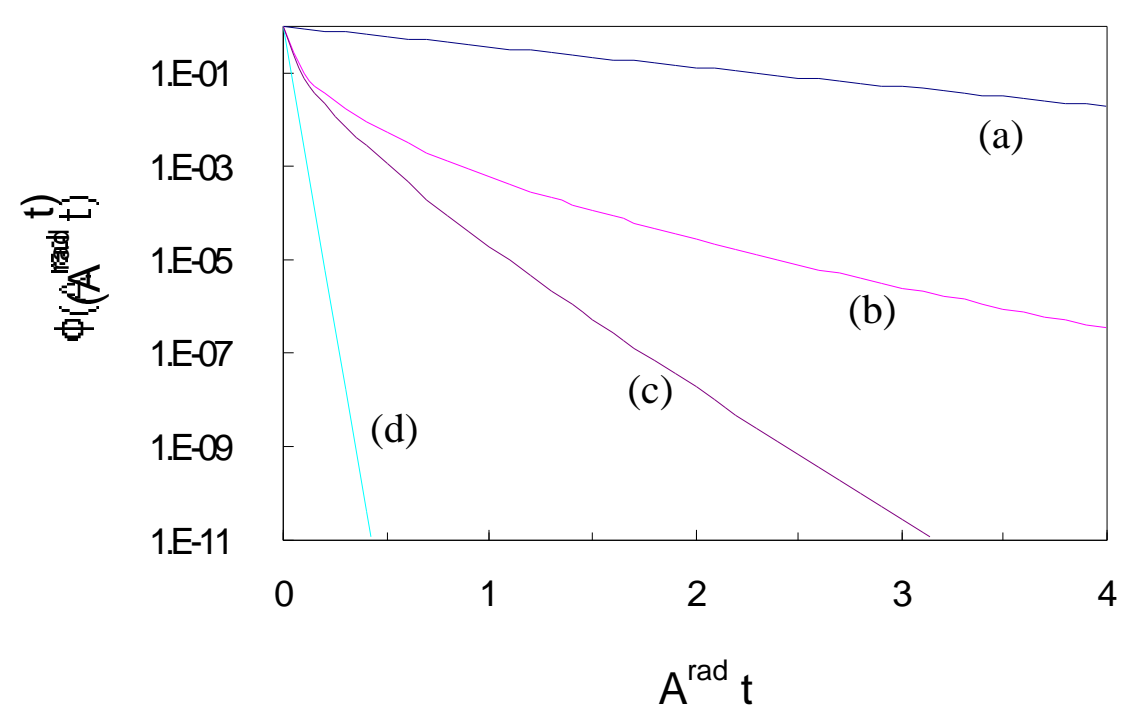

Figure 2.8. Decay curves for the various cases described in the text. (a) represents $\phi_{1}\left(A^{\mathrm{rad}} t\right)$ for $W^{0}=A^{\mathrm{rad}}$. (b) represents $\phi_{2}\left(A^{\mathrm{rad}} t\right)$ with $N_{A} R_{D A}{ }^{3}=1$. (c) represents $\phi_{3}\left(A^{\text {rad }} t\right)$ with $N_{A} R_{D A}{ }^{3}=1$ and $N_{D} R_{D D}{ }^{3}=0.1$. (d) represents $\phi_{4}\left(A^{\text {rad }} t\right)$ with $N_{A} R_{D A}^{3}=1$ and $N_{D} R_{D D}{ }^{3}=3$.

where $\Delta \bar{E}$ is the energy mismatch between a point in the tail of the electronic spectra (arbitrarily fixed at $E_{o}$ ) and the Stokes vibronic spectra, and $\bar{N}$ is the number of phonons required to assist the non-resonant energy transfer $\left(\bar{N}=\Delta \bar{E} / \hbar \omega_{\text {eff }}\right)$. Figure 2.9 displays these concepts. This exponential "tail" has also been described elsewhere in the literature. ${ }^{38,39}$

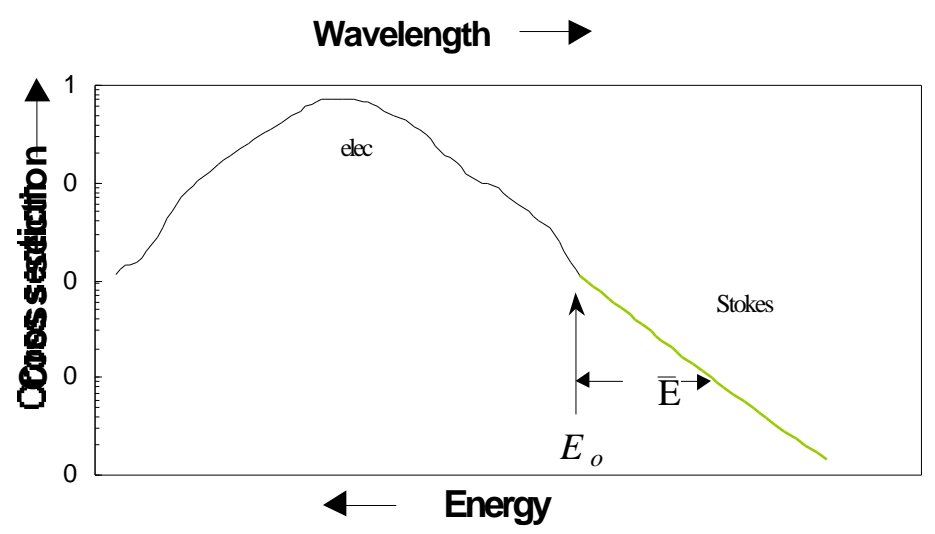

Figure 2.9. The Stokes cross section manifests itself as the exponential tail of the electronic cross section. Note $\sigma^{\text {Stokes }}$ appears as a straight line on the log-scale plot. 
The parameter $\alpha_{S}$ depends only on the host and is given by ${ }^{36}$

$$
\alpha_{S}=\frac{\ln [\bar{N} / 2 g]-1}{h \omega_{e f f}}
$$

where $\omega_{\text {eff }}$ and $g$ are defined as in (2.39).

\subsection{Judd-Ofelt Analysis}

As described at the end of Section 1.3, Judd and Ofelt made the simplifying assumptions that allowed a solution to (1.6). They replaced the energy denominators by a single average denominator $E_{\text {ave }}$ which is independent of $J, J^{\prime}$, and $\varepsilon$ so that $E_{\text {ave }}$ can be pulled out of the summations in (1.6). This assumes that the excited states $|\varepsilon\rangle$ lie far above the states $\langle A|$ and $\left|A^{\prime}\right\rangle$. This assumption is generally valid in rare-earths, although there are some exceptions. The matrix element can now be written

$$
\left\langle A\left|\mathbf{P}_{\rho}^{(1)}\right| A^{\prime}\right\rangle={ }_{t, q} Y(t, q, \rho)\left\langle 4 f^{n}[S L] J\left\|\mathbf{U}^{(t)}\right\| 4 f^{n}\left[S^{\prime} L^{\prime}\right] J^{\prime}\right\rangle,
$$

where

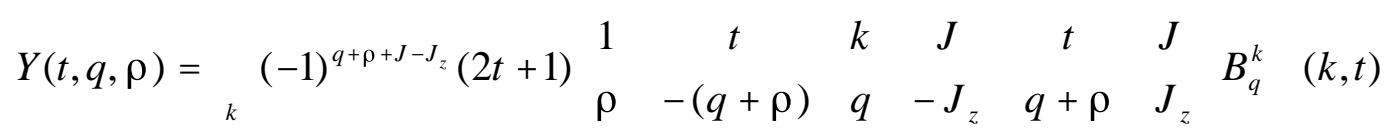

and

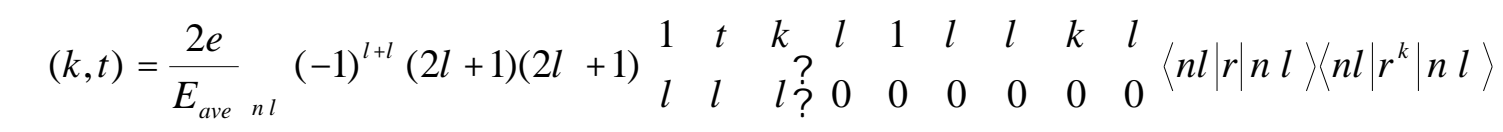

In this equation,

$$
\left\langle n l\left|r^{k}\right| n^{\prime} l^{\prime}\right\rangle={ }_{0}^{\times} R(n l) r^{k} R\left(n^{\prime} l^{\prime}\right) d r
$$


where $R / r$ is the radial portion of the appropriate single-ion wavefunction. Triangular conditions on the 6-j \{\} and 3-j ( ) symbols in (2.60) and (2.61) impose selection rules on the forced electric-dipole transitions as follows:

$$
\Delta l= \pm 1 ; \quad \Delta S=0 ; \quad \Delta L \leq 2 l ; \quad \Delta J \leq 2 l .
$$

Spin-orbit interactions will typically lead to violation of the $\Delta S$ and $\Delta L$ restrictions, but the $\Delta J \leq 6$ rule will usually be valid for $4 f$ electrons, provided there is no " $J$-mixing" so that $J$ remains a "good" quantum number.

The line strength of a transition from the states of the manifold $J$ to those of manifold $J^{\prime}$ is given by

$$
S^{E D}\left(J, J^{\prime}\right)={J J J_{z}^{\prime} \rho}_{\rho}\left|\left\langle A\left|\mathbf{P}_{\rho}^{(1)}\right| A^{\prime}\right\rangle\right|^{2}
$$

Assuming the states of the $J$ manifold are equally occupied, Judd and Ofelt were able to evaluate this sum as

$$
S^{E D}\left(J, J^{\prime}\right)=\Omega_{t=2,4,6}\left|\left\langle 4 f^{n}[S L] J\left\|\mathbf{U}^{(t)}\right\| 4 f^{n}\left[S^{\prime} L^{\prime}\right] J^{\prime}\right\rangle\right|^{2}
$$

where $\left|\left\langle f^{n}[S L] J\left\|U^{(t)}\right\| f^{n}\left[S^{\prime} L^{\prime}\right] J^{\prime}\right\rangle\right|^{2}$ are the squared reduced-matrix elements commonly found in the literature, ${ }^{40}$ and $\Omega_{2}, \Omega_{4}, \Omega_{6}$ are the host-dependent "Judd-Ofelt" intensity parameters given by

$$
\Omega_{t}=(2 t+1) \underset{k, q}{\mid}\left|B_{q}^{k}\right|^{2}(2 k+1)^{-1} Z^{2}(k, t) .
$$

These parameters can be determined by a best fit calculation, since $S^{E D}$ can also be determined from the integrated absorption cross section 


$$
\sigma^{a b s}(\lambda) \frac{d \lambda}{\lambda}=\frac{4 \pi^{2}}{3} \frac{e^{2}}{\hbar c} \frac{1}{2 J+1}\left[\chi_{\sigma}^{E D} S^{E D}+\chi_{\sigma}^{M D} S^{M D}\right],
$$

where $2 J+1$ is the degeneracy of the ground state and $\chi_{\sigma}^{E D}=\left(n^{2}+2\right)^{2} / 9 n$ and $\chi_{\sigma}^{M D}=n$ are the factors that adjust the cross section $\sigma$ for the dielectric and local-field environment for electric and magnetic dipole transitions, respectively. These factors are derived in Appendix B. The method discussed by Krupke ${ }^{41}$ is used to perform the best fit. This method requires the contributions from magnetic dipole transitions to be subtracted from the integrated absorption cross section. The line strength of induced magnetic dipole transitions is given by

$$
S^{M D}=(\hbar / 2 m c)^{2}\left|\left\langle f^{n}[S L] J\|\vec{L}+2 \vec{S}\| f^{n}\left[S^{\prime} L^{\prime}\right] J^{\prime}\right\rangle\right|^{2},
$$

where the bracketed quantities indicate the matrix elements must be computed in the intermediate coupling scheme. These matrix elements however are most easily computed in the LS coupling scheme, ${ }^{42}$ so that coupling coefficients $C(S, L)$ are needed where

$$
\left|4 f^{n}[S L] J\right\rangle=\underset{S, L}{C} C(S, L)\left|f^{n} S L J\right\rangle .
$$

The $C(S, L)$ for $\mathrm{Dy}^{3+}$ were taken from the eigenfunctions of the energy matrix found in Ref. ${ }^{43}$. Magnetic dipole transitions obey the selection rules

$$
\Delta l=0 ; \quad \Delta S=0 ; \quad \Delta L=0 ; \quad|\Delta J| \leq 1 .
$$

Calculations of $S^{M D}$ for $\mathrm{Dy}^{3+}$ and other rare-earth ions are given in Appendix C. Once the line strengths are determined, the spontaneous emission rates and radiative branching ratios for the $\mathrm{J} \rightarrow \mathrm{J}^{\prime}$ transitions can be calculated: 


$$
\begin{gathered}
A_{J J^{\prime}}=\frac{32 \pi^{3} c}{3 \lambda^{3}} \frac{e^{2}}{\hbar c} \frac{1}{2 \mathrm{~J}+1}\left[\chi_{A}^{E D} S^{E D}+\chi_{A}^{M D} S^{M D}\right], \\
A_{J}=A_{J^{\prime}}=1 / \tau_{J}^{\mathrm{rad}}, \\
\beta_{J J^{\prime}}^{\mathrm{rad}}=A_{J J^{\prime}} / A_{J},
\end{gathered}
$$

where $\tau_{\jmath}^{r a d}$ is the radiative lifetime of the level $\mathrm{J}$, and $\chi_{A}^{E D}=n\left(n^{2}+2\right)^{2} / 9$ and $\chi_{A}^{M D}=n^{3}$ are the factors that adjust $A_{J J}$, for the dielectric and local-field environment for electric and magnetic dipole transitions, respectively (see Appendix B). The radiative quantum efficiency $\eta_{\lrcorner}^{r a d}$ is given by

$$
\eta_{J}^{\text {rad }}=\tau_{J}^{\text {meas }} / \tau_{J}^{\text {rad }}
$$

where $\tau_{\mathrm{J}}^{\text {meas }}$ is the measured fluorescence lifetime of level $\mathrm{J}$.

\subsection{Laser Modeling}

To model the temporal profile of the pulsed output of a laser cavity, rate equations can be written for the upper and lower laser level populations and the total number of photons in the cavity. As we will see, these will form a set of coupled differential equations which can be solved by standard techniques. Spatial information will not be included here. Figure 2.10 depicts the processes to be modeled. 


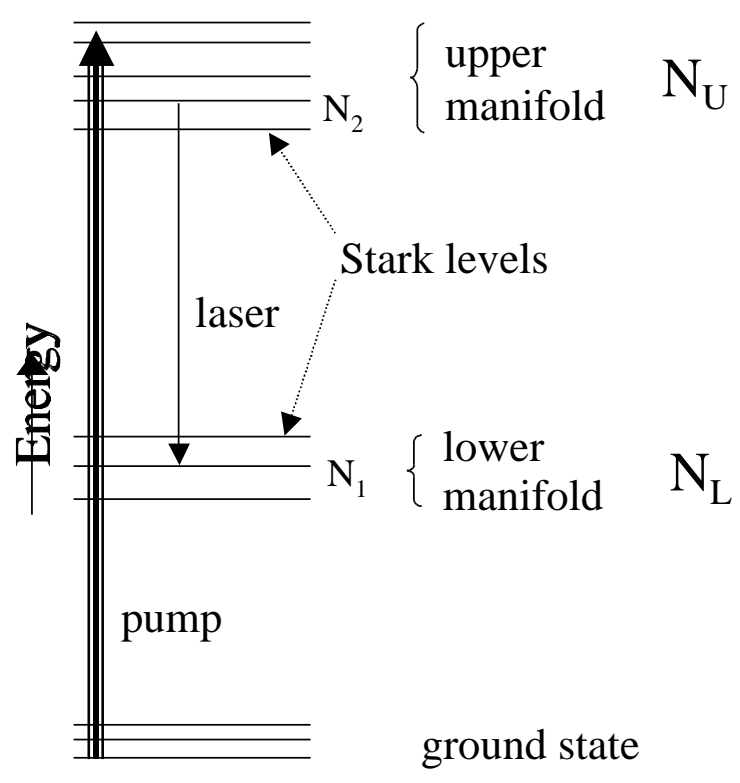

Figure 2.10. Schematic of the energy-level diagram used in the temporal modeling of laser output. Laser action occurs between individual Stark levels with populations $\mathbf{N}_{2}$ and $N_{1}$. Each manifold is assumed to be in thermal equilibrium. The upper and lower manifold have populations $N_{U}$ and $N_{L}$, respectively.

$N_{U}$ and $N_{L}$ represent the upper and lower manifold populations. The laser action is assumed to initiate from a single Stark level (with energy $E_{2}$ and population number $N_{2}$ ) in the upper manifold and terminate on a single Stark level (with energy $E_{1}$ and population number $N_{l}$ ) in the lower manifold. Each manifold is assumed to be in thermal equilibrium at all times, so that $N_{1}=f_{1} N_{L}$ and $N_{2}=f_{2} N_{U}$, where $f_{1}$ and $f_{2}$ are the Boltzmann occupation factors given by (2.23). The rate equations for the upper and lower laser level populations take the form

$$
\begin{aligned}
& \frac{d N_{2}}{d t}=f_{2} \frac{N_{p}}{\tau_{p}} \psi(t)-N_{2} / \tau_{2}-f_{2} \frac{c}{V_{e f f}} \Phi\left(N_{2} \sigma_{21}-N_{1} \sigma_{12}\right), \\
& \frac{d N_{1}}{d t}=f_{1} \frac{b_{21}}{\tau_{2}} N_{2}+f_{1} \frac{c}{V_{e f f}} \Phi\left(N_{2} \sigma_{21}-N_{1} \sigma_{12}\right)-N_{1} / \tau_{1} .
\end{aligned}
$$


Here, $\Phi$ is the cavity photon number, $c$ is the speed of light, $\sigma_{21}$ and $\sigma_{12}$ are the peak emission and absorption cross sections on the laser transition, respectively, $N_{p}$ is the number of ions excited into the upper laser manifold and is given by $N_{p}=E_{i n} / h v_{p}$, where $E_{\text {in }}$ is the absorbed input pump energy, $\psi(t)$ is the temporal form of the pump pulse, normalized so that its integral over time is equal to its pulse width $\tau_{p}, \tau_{2}$ and $\tau_{l}$ are the upper and lower level lifetimes, respectively, $b_{21}$ is the total branching ratio from the upper manifold to the lower manifold, and $V_{\text {eff }}$ is the effective mode volume with which the excited ions interact, which can be written as $V_{\text {eff }}=A_{\text {eff }} l_{m}$, where $l_{m}$ is the length of the gain medium. The rate equation for the cavity photon number is written

$$
\frac{d \Phi}{d t}=\frac{c \sigma_{21}}{V_{e f f}} N_{2}[\Phi+1]-\frac{c \sigma_{12}}{V_{e f f}} N_{1} \Phi-\Phi / \tau_{c},
$$

where in this case the photons interact with the entire cavity, so that $V_{\text {eff }}=A_{\text {eff }} l_{c}$, where $l_{c}$ is the optical length of the cavity. $\tau_{c}$ is the cavity lifetime given by

$$
\tau_{c}=\frac{2 l_{c}}{c(L+T)}
$$

Here, $L$ represents the round-trip internal (passive) losses, and $T$ is the mirror transmission. In general, $A_{\text {eff }}$ will depend upon both the pump and laser mode. For two overlapping lowest-order Gaussian modes with spot-sizes $w_{p}$ and $w_{l}$, respectively, it can be shown ${ }^{44}$ that $A_{\text {eff }}=\frac{1}{2}\left(\pi w_{p}{ }^{2}+\pi w_{l}^{2}\right)$. The "plus one" term in brackets in (2.77) refers to the "extra photon" created from fundamental quantum noise. ${ }^{45}$ This term allows photons to accumulate in the cavity under the initial condition $\Phi(t=0)=0$. The rate 
equations given above can be written in terms of the dimensionless parameters $\phi=$ $\Phi / \Delta N_{t h}, n_{2}=N_{2} / \Delta N_{t h}, n_{1}=N_{l} / \Delta N_{t h}$, and $\tau=t / \tau_{2}$, where

$$
\ddot{A} N_{t h}=\frac{A_{e f f} l_{c}}{c \sigma_{21} \tau_{c}}=\frac{A_{e f f}}{2} \frac{L+T}{\sigma_{21}}
$$

is the population inversion that just makes $d \Phi / d t>0$ (neglecting the noise term). This represents the number of ions that need to be inverted to reach oscillation threshold. We must pump more ions than this, however, since some ions will reside in a different Stark level in the pumped (upper) manifold, and some will be lost to spontaneous emission during the pump pulse. The fraction residing in the upper laser level will be $f_{2}$, and the fraction lost to spontaneous emission during the pump pulse will be $1-\eta_{p}$, where $\eta_{p}$ is the pumping efficiency and will depend upon the pulse shape. The pumping efficiency can be approximated by its upper limit, which is that given for a square pulse with negligible lower level population: ${ }^{46}$

$$
\eta_{p, s}=\frac{1-\exp \left(-\tau_{p} / \tau_{2}\right)}{\tau_{p} / \tau_{2}}
$$

The absorbed pump energy threshold for laser oscillation is then given by $E_{t h}=\ddot{A} N_{t h} h v_{p} / f_{2} \eta_{p}$, which for lowest-order Gaussian pump and laser modes can be written

$$
E_{t h}=\frac{\pi\left(w_{p}^{2}+w_{l}^{2}\right) h v_{p}(L+T)}{4 \sigma^{e m} \eta_{p}}
$$

Here we have used the effective emission cross section $\sigma^{e m}=f_{2} \sigma_{21}$. The effective cross section represents the manifold-manifold cross section that is typically measured, for 
example by (2.20) or (2.26). If we also define a pumping ratio $r=\eta_{p} f_{2} N_{p} / \Delta N_{t h}$ and degeneracy ratio $g=\sigma_{12} / \sigma_{21}$ we have

$$
\begin{gathered}
\frac{d n_{2}}{d \tau}=\frac{r}{\eta_{p}} \frac{\tau_{2}}{\tau_{p}} \psi(\tau)-n_{2}-f_{2} \frac{\tau_{2}}{\tau_{c}} \frac{l_{c}}{l_{m}} \phi\left(n_{2}-g n_{1}\right), \\
\frac{d n_{1}}{d \tau}=f_{1} b_{21} n_{2}+f_{1} \frac{\tau_{2}}{\tau_{c}} \frac{l_{c}}{l_{m}} \phi\left(n_{2}-g n_{1}\right)-\frac{\tau_{2}}{\tau_{1}} n_{1}, \\
\frac{d \phi}{d \tau}=\frac{\tau_{2}}{\tau_{c}} \phi\left[\left(n_{2}-g n_{1}\right)-1\right]+\frac{\tau_{2}}{\tau_{c}} \frac{n_{2}}{\ddot{A} N_{t h}} .
\end{gathered}
$$

These coupled first-order differential equations are well suited to the numerical solution technique known as the fourth-order Runge-Kutta method, ${ }^{47}$ which in turn is well-suited to implementation on standard spreadsheet software. Such a spreadsheet was developed for the analysis of laser output discussed in a future section.

Since the population in level 2 decays only by stimulated emission or spontaneous decay (radiative + nonradiative), an extraction efficiency $\eta_{\text {ext }}$ can be defined as the ratio of the number of stimulated photons to the total number of photons pumped into level 2. The former is given by the integral of the last term in $(2.82)$, while the latter is given by the integral of the first term in (2.82), so that we can write

$$
\eta_{\text {ext }}(r)=\frac{f_{2} \frac{\tau_{2}}{\tau_{c}} \frac{l_{c}{ }_{m}^{\times}}{l_{m}} \phi(\tau)\left[n_{2}(\tau)-g n_{1}(\tau)\right] d \tau}{\frac{r}{\eta_{p}}{ }_{0}^{\times} \frac{\tau_{2}}{\tau_{p}} \psi(\tau) d \tau}=\frac{l_{c}}{l_{m}} \frac{\tau_{2}}{\tau_{c}} \frac{f_{2} \eta_{p}{ }^{\times}}{r} \phi(\tau)\left[n_{2}(\tau)-g n_{1}(\tau)\right] d \tau .
$$

The extraction efficiency depends upon how hard the medium is pumped. The slope efficiency $\eta_{\text {slope }}=d E_{\text {out }} / d E_{\text {in }}$ will be limited by the extraction efficiency as $r \bullet \times$. Fan $^{48}$ 
has shown that if $\tau_{1}<<\tau_{c}, \eta_{e x t}\left(r \vee \times, \tau_{1}<<\tau_{c}\right)=\eta_{p}$. However, for bottlenecked transitions where $\tau_{l} \approx \tau_{c}$ or greater, $\eta_{\text {ext }}(r \vee \times)$ becomes

$$
\eta_{e x t}\left(r \bullet \times, \tau_{1}>\tau_{c}\right)=\frac{f_{2}}{f_{1}+f_{2}} \eta_{p}
$$

The output energy $E_{\text {out }}$ can be written as

$$
E_{\text {out }}(r)=\left(E_{\text {in }}-E_{\text {th }}\right) \eta_{\text {ext }}(r) \frac{v_{l}}{v_{p}} \frac{T}{L+T} \eta_{\bmod e}(r),
$$

where $v_{/} / v_{p}$ is the quantum defect, $T /(L+T)$ is the fraction coupled out of the cavity, and $\eta_{\text {mode }}(r)$ represents a pump-intensity-dependent geometrical factor describing how well the pump mode can extract energy from the laser mode. This will be discussed more in the next section. The slope efficiency can then be written

$$
\eta_{\text {slope }}=\eta_{\text {ext }}(r) \frac{v_{l}}{v_{p}} \frac{T}{L+T} \eta_{\bmod e}(r)
$$

\subsection{Laser Predictions and Analysis}

Formulas for predicting laser threshold and slope efficiencies have been developed for longitudinally pumped solid-state lasers. ${ }^{49,50,51}$ They assume that both the pump and laser modes can be spatially modeled as circular Gaussian beams. The pumping rate is described by $R \psi(r, z)$, where $r$ is the radial coordinate and $z$ is the longitudinal coordinate. The spatial distribution of the pump is contained in $\psi(r, z)$, which is normalized so that its volume integral is unity. It is given by 


$$
\psi(r, z)=\frac{2 \alpha_{p}}{\pi w_{p}{ }^{2}} \exp \overline{\frac{-2 r^{2}}{w_{p}{ }^{2}}} \sqrt[V]{ } \exp \left(-\alpha_{p} z\right),
$$

where $w_{p}$ is the pump-beam waist and $\alpha_{p}$ is the absorption coefficient at $v_{p}$. Similarly the laser beam intensity is given by $I(r, z)=P_{l} \phi(r)$, where $P_{l}$ is the laser power travelling in one direction inside the laser cavity, and

$$
\phi(r)=\frac{2}{\pi w_{l}^{2}} \exp \frac{-2 r^{2}}{w_{l}^{2}} \sqrt[V]{ }
$$

where $w_{l}$ is the laser-beam waist. $\phi(r)$ is normalized with respect to the integral over its cross-sectional area. Both $w_{l}$ and $w_{p}$ are assumed to be constant inside the crystal. For a homogeneously broadened system with saturation, the increase in the laser intensity $I(r, z)$ is given by

$$
\frac{d I(r, z)}{d z}=\frac{G_{0}(r, z) I(r, z)}{1+2 I(r, z) / I_{s a t}},
$$

where $G_{0}(r, z)$ is the unsaturated gain and $I_{s a t}$ is the saturation parameter. This can be derived by solving differential equations similar to (2.75) and (2.76) in the steady-state. The factor of 2 in the denominator accounts for both passes of the one-way laser intensity $I(r, z)$ through the gain medium. The unsaturated gain is given by $G_{0}(r, z)=\sigma_{21} \Delta N(r, z)$, where $\sigma_{21}$ is the peak cross section for the $2 \rightarrow 1$ laser transition, and $\Delta N(r, z)$ is the population inversion. Assuming the lower level lifetime is sufficiently fast that the lower level population is negligible compared to the upper level population, in the steady state we must have (e.g. equation (2.75) with $\Phi=0$ )

$$
\Delta N(r, z)=f_{2} \tau_{2} R \psi(r, z),
$$


where $f_{2}$ is the Boltzmann occupation factor of the upper laser level given by (2.23), and $\tau_{2}$ is the upper laser level lifetime. The saturation parameter is given by

$$
I_{s a t}=\frac{h v_{l}}{\sigma^{e m} \tau_{2}}
$$

where we have used the effective cross section $\sigma^{e m}=f_{2} \sigma_{21}$. If the losses in the laser cavity are small, the oscillation condition (round-trip gain equals round-trip loss) can be written

$$
\underset{\substack{\text { round } \\ \text { trip }}}{d P_{l}=2}{ }_{0}^{l_{m}} \frac{d P_{l}(z)}{d z} d z=P_{l}(L+T),
$$

where the round-trip passive losses are characterized by $L, T$ is the total output coupling, and $l_{m}$ is the length of the gain medium. $P_{l}$ (without the argument $\mathrm{z}$ ) represents an average laser power and indicates that in the low-loss, low-gain approximation the laser power remains largely constant along the laser axis. As a function of $z$, the single-pass laser power $P_{l}(z)$ is given by

$$
P_{l}(z)=2 \pi_{0}^{\times} I(r, z) r d r
$$

The oscillation condition (2.94) can then be written

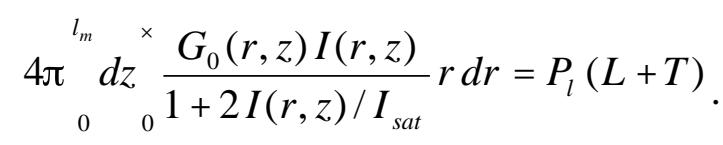


Inserting the relations for $G_{0}(r, z)$ and $I(r, z)$, and performing the integration over $\mathrm{z}$ gives

$$
F=\frac{\times}{{ }_{0}} \frac{\exp \left[-\left(1+a^{2}\right) x\right]}{1+S \exp \left(-a^{2} x\right)} d x \sqrt{ },
$$

where $a=w_{p} / w_{l}, x=2 r^{2} / w_{p}{ }^{2}, F$ is a normalized pump power given by

$$
F=\frac{4}{(L+T)} \frac{v_{l}}{v_{p}} \frac{P_{i n}}{P_{s a t}}
$$

$S$ is a normalized laser output power given by

$$
S=\frac{4}{T} \frac{P_{o u t}}{P_{s a t}}
$$

where we have used $P_{\text {out }}=T P_{l}$, and

$$
P_{s a t}=\pi w_{l}^{2} I_{s a t}
$$

An expression for oscillation threshold can be derived by solving (2.97) for the case $S=$ 0 . In this case, $F(S=0)=F_{t h}=1+a^{2}$, or by (2.98) the threshold (absorbed) pump power is

$$
P_{\text {in }}^{\text {th }}=P_{\text {sat }} \frac{\boldsymbol{v}_{p}}{v_{l}} \sqrt[v]{\frac{1+a^{2}}{4}} \sqrt{ }(L+T),
$$

or by (2.93) and (2.100)

$$
P_{i n}^{t h}=\frac{\pi w_{l}^{2}\left(1+a^{2}\right) h v_{p}(L+T)}{4 \sigma^{e m} \tau_{2}} .
$$


Likewise, the laser slope efficiency, which is defined as $\eta_{\text {slope }}=d P_{\text {out }} / d P_{\text {in }}$, can be determined from (2.98) and (2.99) as

$$
\eta_{\text {slope }}=\frac{d S}{d F} \frac{v_{l}}{v_{p}} \frac{T}{(L+T)}
$$

Here, $d S / d F$ represents a geometrical factor characterizing the laser-mode and pumpmode overlap efficiency. That is, $d S / d F$ gives the efficiency with which absorbed photons (in excess of those requires to reach threshold) are converted to photons inside the cavity. $d S / d F$ is identified as $\eta_{\text {mode }}$ from the previous section. It can be obtained by differentiating (2.97) with respect to $S$ :

$$
\frac{d S}{d F}=F^{2}{ }_{0}^{\times} \frac{\exp \left[-\left(1+2 a^{2}\right) x\right]}{\left[1+S \exp \left(-a^{2} x\right)\right]^{2}} d x \sqrt{ } \sqrt{ }^{-1},
$$

where $F$ is related to $S$ by (2.97). A numerically generated plot of $d S / d F$ as a function of $P_{i n} / P_{t h}$ for different values of $a=w_{p} / w_{l}$ is shown in Figure 2.11.

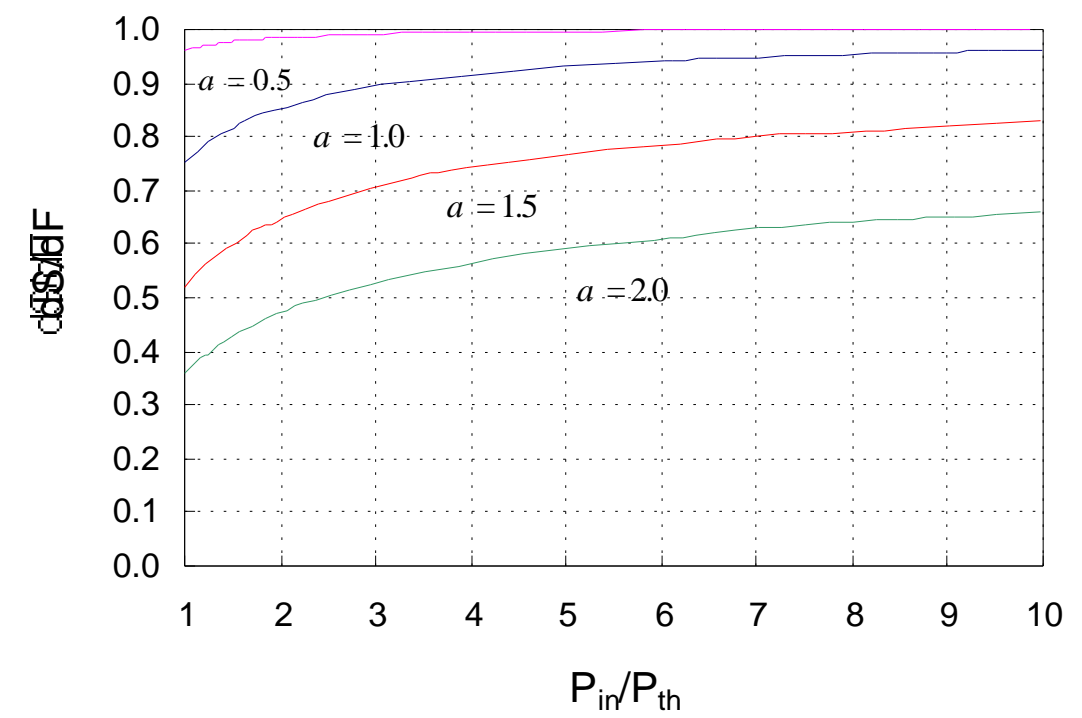

Figure 2.11. Numerically generated plot of the normalized slope efficiency $d S / d F$ as a function of input power $P_{\text {in }} / P_{\text {th }}$. Note $d S / d F \approx 1$ for $a<0.5$. The parameter $a$ is defined as $w_{p} / w_{l} . d S / d F$ is identified as $\eta_{\text {mode }}$ of Section 2.5. 
Two cases of interest can be solved analytically. For $a \rightarrow 0, d S / d F=1$. This indicates the mode extraction efficiency $d S / d F$ is greatest when the pump-mode waist is a small fraction of the laser-mode waist. When $S \rightarrow 0$, we get

$$
\left.\frac{d S}{d F}\right|_{S \diamond 0}=\frac{1+2 a^{2}}{\left(1+a^{2}\right)^{2}}
$$

This is the extraction efficiency at threshold, which represents a lower bound for $d S / d F$. In the previous section we found that for bottlenecked lasers an extra term must be included in the slope efficiency. With this factor included, the slope efficiency valid for bottlenecked laser transitions takes the form

$$
\eta_{\text {slope }}=\frac{d S}{d F} \frac{f_{2}}{f_{1}+f_{2}} \eta_{p} \frac{v_{l}}{v_{p}} \frac{T}{L+T}
$$

Equations (2.102) and (2.103) can be used as analysis tools when laser threshold and slope efficiency data can be measured. Re-writing (2.102) as

$$
P_{i n}^{t h}=P_{0}(L+T)
$$

where

$$
P_{0}=\frac{\pi w_{l}^{2}\left(1+a^{2}\right) h v_{p}}{4 \sigma^{e m} \tau_{2}} .
$$

By plotting $P_{i n}^{\text {th }}$ vs. $T$, we can determine the intracavity losses $L$ by a straight-line fit. The slope of such a line is given by $m=P_{0}$, while the intercept is given by $b=P_{0} L$. The round-trip intracavity losses $L$ are given by $L=b / m$. This plot is known as a FindlayClay plot. ${ }^{52}$ 
In a similar manner, Caird ${ }^{53}$ re-wrote $(2.103)$ as

$$
\eta_{\text {slope }}^{-1}=\eta_{0}^{-1}\left(1+L T^{-1}\right)
$$

where $\eta_{0}$ represents the limiting slope efficiency in the absence of passive losses. By fitting a straight-line to a "Caird" plot of $\eta_{\text {slope }}{ }^{-1}$ vs. $T^{-1}$, we can determine $L$ and $\eta_{0}$ from its slope $m$ and intercept $b$. The slope is given by $m=L / \eta_{0}$ and the intercept is given by $b$ $=1 / \eta_{o}$. Thus $L=m / b$ and $\eta_{0}=1 / b$. 


\section{Theoretical Investigations}

Some issues surfaced in the course of this research that required development beyond the historical basis. These include a method to measure radiative quantum efficiencies and multiphonon decay rates, a method to determine emission cross section from laser performance data, and the analysis of the effect of a wedged sample in a laser cavity.

\subsection{Quantum Efficiency Model}

With ground-state absorptions at $2.8,1.7,1.3,1.1$, and $0.9 \mu \mathrm{m}$, the low-lying energy levels in $\mathrm{KPb}_{2} \mathrm{Cl}_{5}: \mathrm{Dy}^{3+}$ are accessible by direct laser excitation (see Figure 3.1).

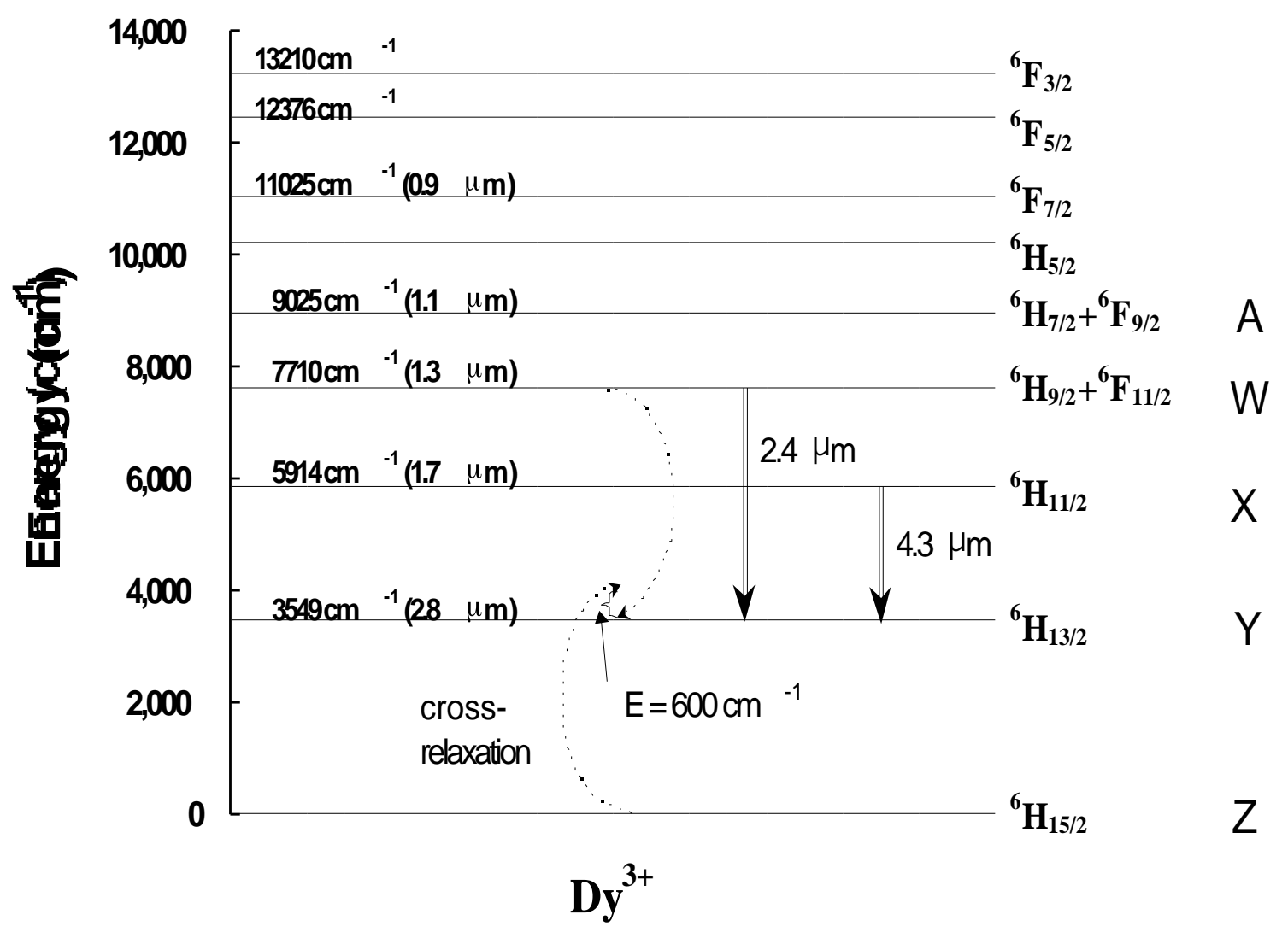

Figure 3.1. Energy level diagram of $\mathrm{Dy}^{3+}: \mathrm{KPb}_{2} \mathrm{Cl}_{5}$. The letter designations of the levels are taken after Dieke [15]. The phonon-assisted energy transfer pathway is indicated. Note this pathway actually involves two neighboring ions: a donor ion in the W-level and an acceptor ion in the Zlevel interacting to promote both ions into the Y-level. 
By exciting each level individually and examining the relative emission from each lowerlying level, the radiative quantum efficiencies $\left(\eta^{\mathrm{rad}}\right.$, defined as the radiative emission rate divided by the total (radiative + nonradiative) emission rate) of these levels can be determined. In practice this has been done by measuring ratios of emission rate intensities $(\phi)$, as we shall see. The concept is pedagogically illustrated in Figure 3.2 for a hypothetical 3-level system. Figure 3.2a shows the energy levels, indicating the pump level (level 3) and the possible output wavelengths $\lambda_{3}$ and $\lambda_{2}$.

(a)

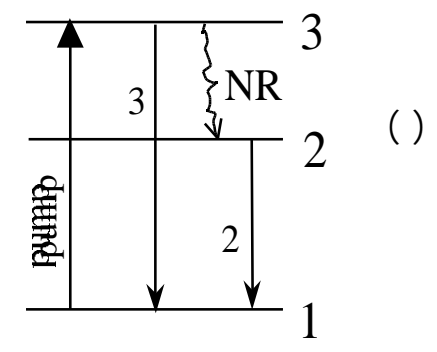

(c)

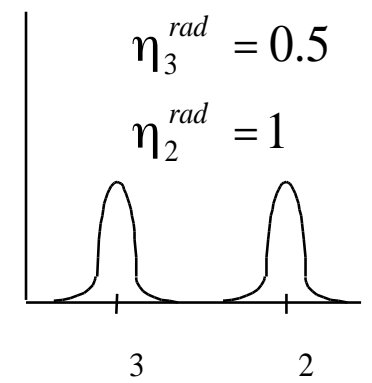

(b)

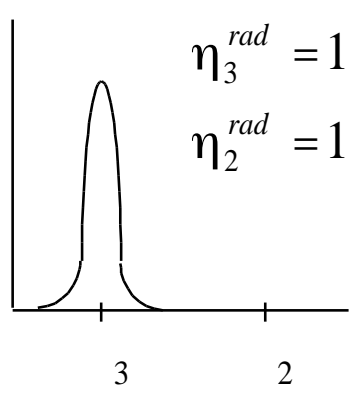

(d)

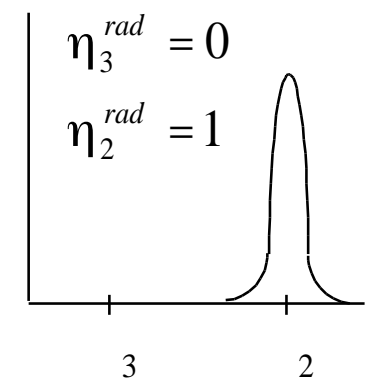

Figure 3.2. Pedagogical illustration of the concept of the radiative quantum efficiency model. a) Level 3 is pumped directly, and we assume $\eta_{2}^{\mathrm{rad}}=1$ and $\beta_{32}^{\mathrm{rad}}=\mathbf{0}$. The wavy arrow indicates nonradiative decay. Hypothetical emission rates $\phi$ (arb. photons/sec) are shown as a function of wavelength $\lambda$ for the cases $b$ ) $\eta_{3}^{\text {rad }}=\mathbf{1}$, c) $\eta_{3}^{\text {rad }}=\mathbf{0 . 5}$, and d) $\eta_{3}^{\text {rad }}=\mathbf{0}$. 
For this example we assume $\eta_{2}^{\text {rad }}=1$, and that $\beta_{32}^{\text {rad }}=0$ so that only nonradiative decay connects level 3 to level 2. Figure 3.2b, Figure 3.2c, and Figure 3.2d show the emission spectra (photon emission rate $\phi$ vs. wavelength $\lambda$ ) in cases where $\eta_{3}^{\text {rad }}=1,0.5$, and 0 , respectively. For this example, it is apparent that $\eta_{3}^{\text {rad }}=\phi_{3} /\left(\phi_{2}+\phi_{3}\right)=\left(1+\phi_{2} / \phi_{3}\right)^{-1}$, where $\phi_{\mathrm{J}}$ is the photon emission rate for level $\mathrm{J}$ represented by the emission peak at $\lambda_{\mathrm{J}}$.

In the following more general model we assume that decay from each level is possibly influenced by radiative, multiphonon, and concentration-dependent transfer processes only, and that multiphonon decay occurs only to the next lowest energy level. Specifically, the cross-relaxation process shown in Figure 3.1 has been assumed. The justification for this assumption will be discussed in a later section. This method is an extension of that used by Quimby, et. al. ${ }^{54}$ The results of this analysis will be used to calculate accurate emission cross sections and multiphonon decay rates which impact the potential use of $\mathrm{KPb}_{2} \mathrm{Cl}_{5}: \mathrm{Dy}^{3+}$ as a long-wavelength laser medium. The model is based on solutions to steady-state rate equations. It has been assumed that the pump intensities involved $\left(\mathrm{I}_{\text {pump }} \sim 10 \mathrm{~W} / \mathrm{cm}^{2}\right)$ do not significantly deplete $\left(\mathrm{I}_{\mathrm{sat}} \sim 10 \mathrm{~kW} / \mathrm{cm}^{2}\right)$ the groundstate population. Denoting ${ }^{P_{N}}$ as the population density of the $\mathrm{J}$ level $(\mathrm{J}=\mathrm{A}, \mathrm{W}, \mathrm{X}, \mathrm{Y})$ under direct excitation of the $P$ level $(P=A, W, X)$ (see Figure 3.3), the steady-state rate equations of levels $\mathrm{W}, \mathrm{X}$, and $\mathrm{Y}$ take the form

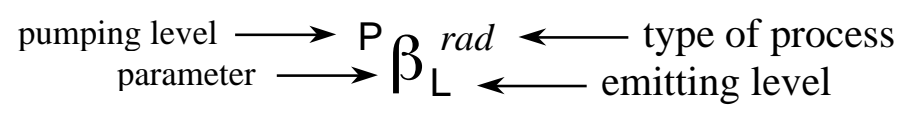

Figure 3.3. Notation used in this section. The pre-superscript refers to the level that was pumped and the post-subscript refers to the emitting level. The post-superscript refers to the nature of the process involved. 


$$
\begin{gathered}
\frac{d\left({ }^{\mathrm{w}} N_{\mathrm{w}}\right)}{d t}=0={ }^{\mathrm{w}} R-{ }^{\mathrm{w}} N_{\mathrm{w}} / \tau_{\mathrm{w}}, \\
\frac{d\left({ }^{\mathrm{w}} N_{\mathrm{x}}\right)}{d t}=0=b_{\mathrm{wx}}{ }^{\mathrm{w}} N_{\mathrm{w}} / \tau_{\mathrm{w}}-{ }^{\mathrm{w}} N_{\mathrm{x}} / \tau_{\mathrm{x}}, \\
\frac{d\left({ }^{\mathrm{w}} N_{\mathrm{Y}}\right)}{d t}=0=\left(b_{\mathrm{wY}}+\eta_{\mathrm{w}}^{c} \beta_{\mathrm{wr}}^{c}\right){ }^{\mathrm{w}} N_{\mathrm{w}} / \tau_{\mathrm{w}}+b_{\mathrm{xY}}{ }^{\mathrm{w}} N_{\mathrm{x}} / \tau_{\mathrm{x}}-{ }^{\mathrm{w}} N_{\mathrm{Y}} / \tau_{\mathrm{Y}}
\end{gathered}
$$

when the $\mathrm{W}$ level is directly pumped, and

$$
b_{\mathrm{J} J^{\prime}}=\eta_{\mathrm{J}}^{\mathrm{rad}} \beta_{\mathrm{J} J^{\prime}}^{\mathrm{rad}}+\eta_{\mathrm{J}}^{M P} \beta_{\mathrm{J} J^{\prime}}^{M P}+\eta_{\mathrm{J}}^{c} \beta_{\mathrm{J} J^{\prime}}^{c}
$$

$\left(\eta_{J}^{\text {rad }}+\eta_{J}^{M P}+\eta_{\jmath}^{c}=1\right)$ represents the total branching ratio through downward relaxation from level $\mathrm{J}$ to level $\mathrm{J}^{\prime}$ via all processes (radiative, multiphonon, and cross-relaxation), ${ }^{\mathrm{w}} R$ is the rate at which level $\mathrm{W}$ is pumped, and $\tau_{J}$ is the total fluorescence lifetime of the level J. The second term in parentheses in (3.3) represents the energy transfer from the ground state to the $Y$ level in the cross-relaxation pathway depicted in Figure 3.1. The quantum efficiencies and branching ratios are all defined similarly: $\eta_{\mathrm{J}}^{a}=W_{\mathrm{J}}^{a} / W_{\mathrm{J}}$, $\beta_{\mathrm{JJ}^{\prime}}^{a}=W_{\mathrm{J}}^{a} / W_{\mathrm{J}}^{a}, a=$ radiative (rad), multiphonon (MP), or cross-relaxation (c). The cross-relaxation fraction $\eta_{\mathrm{w}}^{c}$ (the fraction of the $\mathrm{W}$-level population decaying by crossrelaxation) is determined from the measured values of $W_{\mathrm{w}}$ and $W_{\mathrm{w}}^{0}$ by use of (2.47):

$$
\eta_{\mathrm{w}}^{c} \ldots W_{\mathrm{w}}^{c} / W_{\mathrm{w}}=1-W_{\mathrm{w}}^{0} / W_{\mathrm{w}} .
$$

The following assumptions were used in this analysis: 
- $\quad \beta_{J^{\prime}}^{M P}=1$ if $\mathrm{J}^{\prime}$ is the next level below $\mathrm{J}, \beta_{\mathrm{J}^{\prime}}^{M P}=0$ otherwise (multiphonon emission occurs only to the next lowest energy level)

- $\quad \beta_{\mathrm{wr}}^{c}=1$ (concentration-dependent energy transfer from the $\mathrm{W}$ level occurs only to the $\mathrm{Y}$ level)

- $\quad \eta_{\mathrm{Y}}^{M P}=0$ (the $\mathrm{Y}-\mathrm{Z}$ gap is sufficiently large (> 15 phonons) to inhibit all multiphonon decay from the $\mathrm{Y}$ level)

Solving the rate equations for the individual populations ${ }^{w} N_{J}$ in the steady-state, and writing the radiative emission rate (photons/sec) from level $\mathrm{J}$ as $\left[{ }^{\mathrm{w}} N_{\mathrm{J}} / \tau_{\mathrm{J}}\right] \eta_{\lrcorner}^{\mathrm{rad}}$, we have:

$$
\begin{gathered}
{\left[{ }^{\mathrm{w}} N_{\mathrm{w}} / \tau_{\mathrm{w}}\right] \eta_{\mathrm{w}}^{\mathrm{rad}}={ }^{\mathrm{w}} R \eta_{\mathrm{w}}^{\mathrm{rad}},} \\
{\left[{ }^{\mathrm{w}} N_{\mathrm{x}} / \tau_{\mathrm{x}}\right] \eta_{\mathrm{x}}^{\mathrm{rad}}={ }^{\mathrm{w}} R b_{\mathrm{wx}} \eta_{\mathrm{x}}^{\text {rad }},} \\
{\left[{ }^{\mathrm{w}} N_{\mathrm{y}} / \tau_{\mathrm{y}}\right] \eta_{\mathrm{Y}}^{\mathrm{rad}}={ }^{\mathrm{w}} R\left(b_{\mathrm{wr}}+\eta_{\mathrm{w}}^{c} \beta_{\mathrm{wr}}^{c}+b_{\mathrm{wx}} b_{\mathrm{xy}}\right) \eta_{\mathrm{Y}}^{\text {rad }} .}
\end{gathered}
$$

The radiative emission rate can also be determined from the blackbody-calibrated emission spectrum ${ }^{\mathrm{P}} I(\lambda)$ (units of arbitrarily scaled $\mathrm{W} / \mathrm{nm}$ ) resulting from direct excitation of the $P$ level $(P=A, W, X)$ since

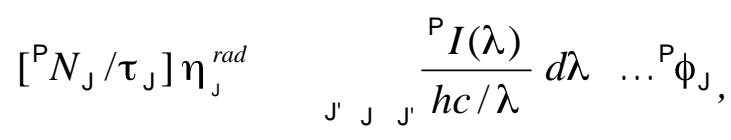

where the integral is carried out over all bands $\mathrm{J} \rightarrow \mathrm{J}^{\prime}$ where $\mathrm{J}^{\prime}$ lies below $\mathrm{J}$. Thus ${ }^{\mathrm{P}} \phi_{\mathrm{J}}$ represents the radiative emission rate from level $\mathrm{J}$ after level $\mathrm{P}$ is pumped, and can be determined experimentally (to within a proportionality constant) from the emission spectrum ${ }^{\mathrm{P}} I(\lambda)$. The radiative branching ratios can also be determined experimentally 
from ${ }^{P} I(\lambda)$. From (3.9), for a single transition $\mathrm{J} \rightarrow \mathrm{J}^{\prime}$ with radiative branching ratio $\beta_{\mathrm{J}}^{\mathrm{rad}}$, we have

$$
\beta_{\mathrm{J} J^{\prime}}^{\mathrm{rad}}=\frac{{ }_{\mathrm{J} \bullet \mathrm{J}^{\prime}}^{\mathrm{P}} I(\lambda) \lambda d \lambda}{{ }_{\mathrm{J}}^{\mathrm{P}} I(\lambda) \mathrm{J}^{\prime}}
$$

Notice that the form of (3.10) makes the value of the branching ratio invariant to the pumping level $\mathrm{P}$.

Arbitrary constants arising from collection efficiency and absolute intensity calibrations of the system are eliminated by taking ratios of relations (3.6), (3.7) and (3.8), and employing (3.9). Labeling the ratio of the radiative emission rate from the $\mathrm{J}^{\text {th }}$ level to the radiative emission rate from the $K^{\text {th }}$ level as ${ }^{\mathrm{P}} \phi_{\mathrm{J} / \mathrm{K}}$ (i.e. ${ }^{\mathrm{P}} \phi_{\mathrm{J} / \mathrm{K}} \equiv{ }^{\mathrm{P}} \phi_{\mathrm{J}} /{ }^{{ }} \phi_{K}$ ), we obtain

$$
\begin{aligned}
& { }^{\mathrm{w}} \phi_{\mathrm{x} w} \cdots \frac{{ }^{\mathrm{w}} \phi_{\mathrm{x}}}{{ }^{\mathrm{w}} \phi_{\mathrm{w}}}=\frac{b_{\mathrm{wx}} \eta_{\mathrm{x}}^{\mathrm{rad}}}{\eta_{\mathrm{w}}^{\mathrm{rad}}} \\
& { }^{{ }^{w}} \phi_{Y,} \cdots \frac{{ }^{w} \phi_{\mathrm{Y}}}{{ }^{w} \phi_{\mathrm{w}}}=\frac{\left(b_{\mathrm{wr}}+\eta_{\mathrm{w}}^{c} \beta_{\mathrm{wr}}^{c}+b_{\mathrm{wX}} b_{\mathrm{XY}}\right) \eta_{\mathrm{Y}}^{\mathrm{rad}}}{\eta_{\mathrm{w}}^{\text {rad }}} .
\end{aligned}
$$

In a similar manner we can write the rate equations for the levels $X$ and $Y$ when level $X$ is directly pumped, and arrive at the relation

$$
{ }^{\mathrm{x}} \phi_{\mathrm{Y} / \mathrm{X}} \cdots \frac{{ }^{\mathrm{x}} \phi_{\mathrm{Y}}}{{ }_{\mathrm{x}} \phi_{\mathrm{X}}}=\frac{b_{\mathrm{XY}} ? \eta_{\mathrm{Y}}^{\mathrm{rad}}}{\eta_{\mathrm{X}}^{\mathrm{rad}}} .
$$


Relations (3.11),(3.12), and (3.13) (in conjunction with (3.4)) represent three equations for the three unknowns $\eta_{\mathrm{w}}^{\text {rad }}, \eta_{\mathrm{x}}^{\text {rad }}$, and $\eta_{\mathrm{Y}}^{\text {rad }}$, allowing a unique determination of these parameters. The solutions are:

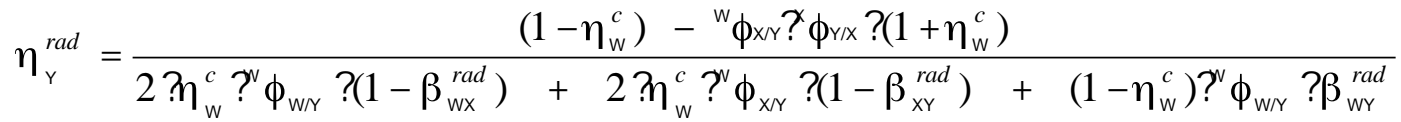

$$
\begin{aligned}
& \eta_{\mathrm{X}}^{\text {rad }}=\frac{\eta_{\mathrm{Y}}^{\text {rad }}}{\mathrm{x}_{\phi_{\mathrm{Y} X}+\eta_{\mathrm{Y}}^{\text {rad }} ?\left(1-\beta_{\mathrm{XY}}^{\text {rad }}\right)}}, \\
& \eta_{\mathrm{w}}^{\mathrm{rad}}=\frac{\eta_{\mathrm{x}}^{\mathrm{rad}} ?\left(1-\eta_{\mathrm{w}}^{c}\right)}{{ }^{\mathrm{w}} \phi_{\mathrm{xw}}+\eta_{\mathrm{x}}^{\text {rad }} ?\left(1-\beta_{\mathrm{wx}}^{r a d}\right)} .
\end{aligned}
$$

The quantum efficiency for the A level can also be determined from a similar analysis. Including the $A$ level in the rate equation analysis, where it now becomes the level directly pumped, and proceeding as before we arrive at the expression

$$
\eta_{\mathrm{A}}^{\text {rad }}=\frac{\eta_{\mathrm{w}}^{\text {rad }}}{{ }^{\mathrm{A}} \phi_{\mathrm{W} / \mathrm{A}}+\eta_{\mathrm{W}}^{\text {rad }} ?\left(1-\beta_{\mathrm{AW}}^{\text {rad }}\right)} .
$$

Finally, it is worthwhile to note that in the absence of energy transfer (as for low $\mathrm{Dy}^{3+}$ concentration), (3.14) takes on the much simpler form

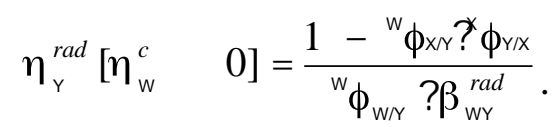

\subsection{Emission cross section from laser performance analysis}

A novel technique to determine the emission cross section of a laser transition can be developed from the results of Section 2.6. The emission cross section is related to the 
threshold pump power $P_{t h}$ of a laser via (2.102), so we expect cross section data could be extracted from laser threshold data. As indicated in Figure 3.4, however, $P_{t h}$ is dependent upon the intracavity losses, which are typically not known with sufficient accuracy. Figure 3.4 also indicates that the intercept of the slope efficiency curve $\left(P_{\text {out }}\right.$ vs. $\left.P_{\text {in }}\right)$ with the $P_{\text {out }}$ axis is independent of the intracavity losses. The value of this intercept is given by the simple formula $P_{\text {out }}=\eta_{\text {slope }}\left(P_{i n}-P_{\text {th }}\right)$ as $\eta_{\text {slope }} \cdot P_{t h}$.

If we examine the product of the threshold and slope efficiency of a laser transition (given by (2.102) and (2.103), respectively), we see that this product is indeed independent of the passive losses $L$ in the resonator and given by

$$
P_{t h} ? \eta_{\text {slope }}=\frac{\pi w_{l}^{2}\left(1+a^{2}\right) h v_{l}}{4 \sigma^{e m} \tau_{2}} \frac{d S}{d F} T .
$$

In fact, the data does not need to reflect absorbed power, since any factors used to correct for pump absorption will also affect the slope efficiency in a reciprocal manner, so that the product of the threshold and slope remains unaffected.

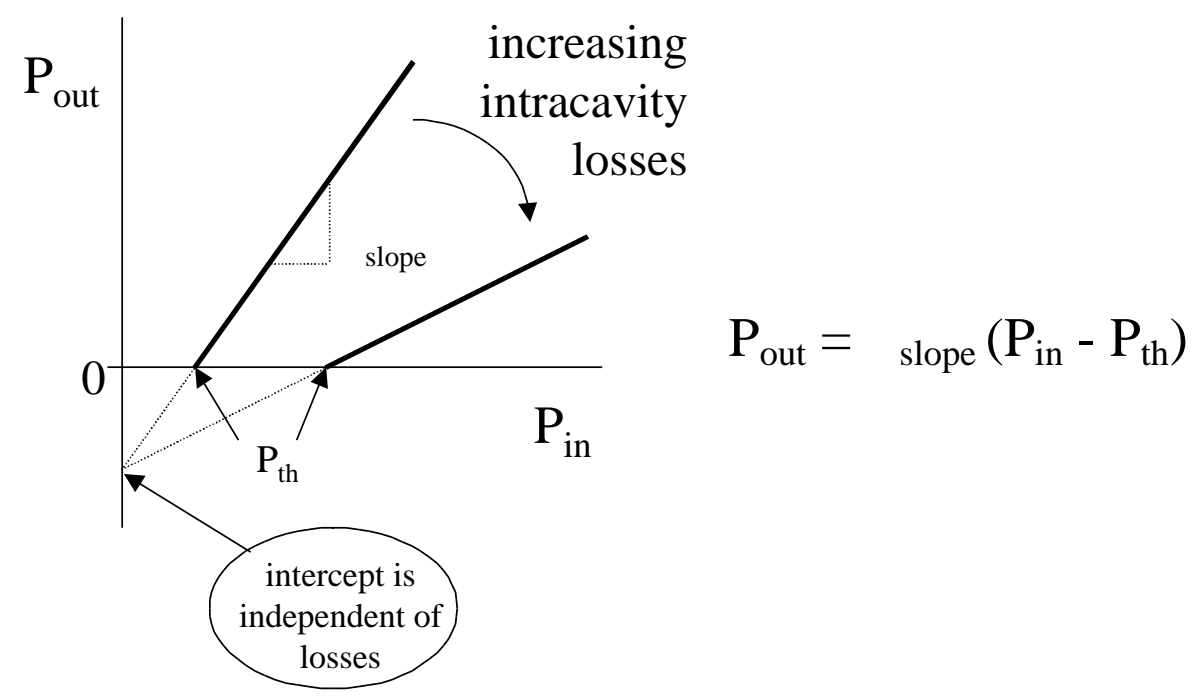

Figure 3.4. Illustration of the threshold-slope product. As evidenced by the equation, the negative of the threshold-slope product is the intercept of the slope-efficiency curve at zero input. This remains independent of intracavity losses $L$ since as these losses are increased, the slope-efficiency curve simply pivots about the intercept. 
For $a<\sim 0.5$ and/or $F \sim F_{t h},(2.105)$ is a good approximation to $d S / d F$ (see Figure 2.11, page $2.6-61)$. In this case we can write

$$
P_{\text {th }} ? \eta_{\text {slope }} \cup \frac{\pi w_{l}^{2}}{\lambda_{l}} \frac{1+2 a^{2}}{1+a^{2}} \frac{h c}{4 \tau_{2}} \frac{1}{\sigma^{e m}} T .
$$

The first term on the right-hand side is the Rayleigh range of the laser mode, which can be determined strictly from the resonator geometry. For $a<<1$, the geometrical factor (second term) is nearly unity. Thus by varying the output coupling $T$ and plotting $P_{t h} ? \eta_{\text {slope }}$ vs. $T$, we can determine the effective cross section $\sigma^{e m}$ from the slope of the straight-fit line if the upper state lifetime $\tau_{2}$ is known. Conversely, if the cross section is known, any of the other factors in (3.19) can be determined, such as $d S / d F$. The key advantage to this method over the $\mathrm{F} \preccurlyeq$ chtbauer-Ladenburg method is that knowledge of the radiative lifetime is not needed. This parameter is often difficult to determine experimentally. In fact, this new method can be used to determine the radiative lifetime by use of the $\mathrm{F} \sqrt{ }$ chtbauer-Ladenburg relation when the cross section is known.

To illustrate this method, measurements were made on a standard laser material, namely $\mathrm{Nd}^{3+}: Y A G$, where the upper state lifetime was measured to be $250 \mu$ s. CW laser action was achieved at $1064 \mathrm{~nm}$ in a 5-cm confocal cavity, pumped by $808-\mathrm{nm}$ radiation from a Argon-ion pumped Ti:Sapphire laser. A lens focussed the input beam to a spot size of $25 \mu \mathrm{m}$, making $a=0.27$. The laser performance data is shown in Table 3.1, and a plot of $P_{t h} \cdot \eta_{\text {slope }}$ vs. $T$ is shown in Figure 3.5. From the slope of the straight-line fit we conclude the effective cross section to be $\sigma^{e m}=27.6 \pm 1.4 \times 10^{-20} \mathrm{~cm}^{2}$. 
Table 3.1. Transmission, threshold, and slope efficiency data for Nd:YAG operating at $1.064 \mu \mathrm{m}$.

\begin{tabular}{|c|c|c|c|}
\hline $\mathbf{T}(\boldsymbol{\%})$ & $\mathbf{P}_{\text {th }}(\mathbf{m W})$ & $\eta_{\text {slope }}$ & $\mathbf{P}_{\text {th }} \cdot \eta_{\text {slope }}(\mathbf{m W})$ \\
\hline \hline 1.23 & 8.59 & 0.279 & 2.397 \\
\hline 2.55 & 13.50 & 0.358 & 4.828 \\
\hline 5.00 & 22.61 & 0.425 & 9.616 \\
\hline
\end{tabular}

This agrees well with measurements made by $\mathrm{Krupke}^{55}$ using the $\mathrm{F} \sqrt{ } \mathrm{chtb}$ tbuer-Ladenburg relation (2.20), indicating a value of $28 \times 10^{-20} \mathrm{~cm}^{2}$.

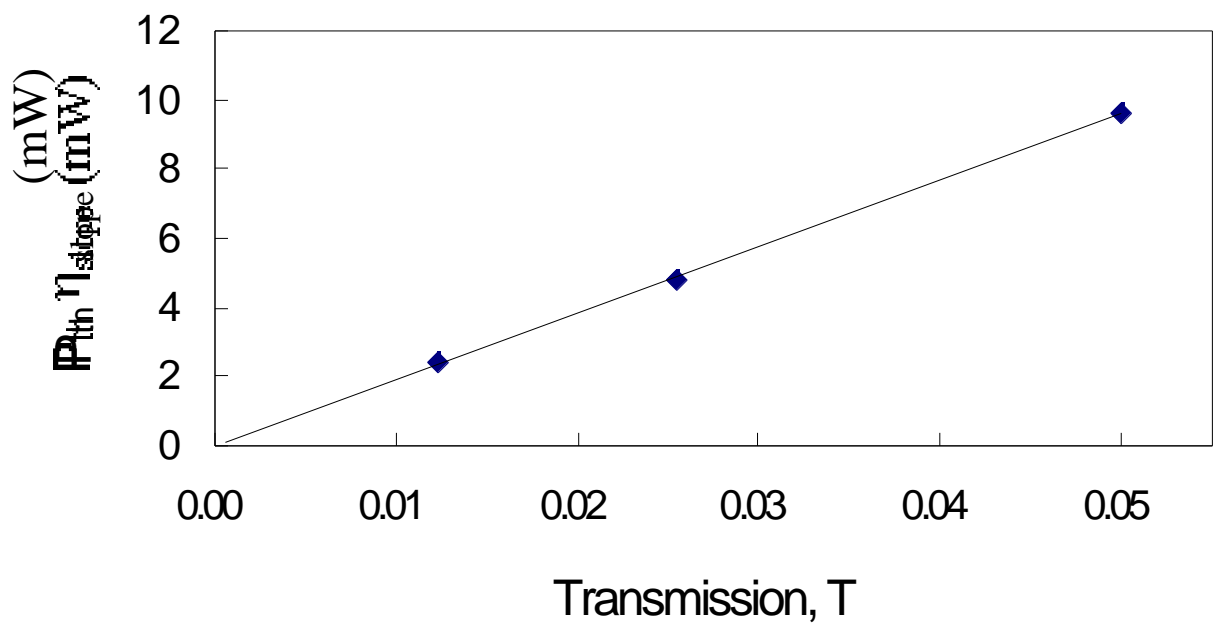

Figure 3.5. The threshold-slope product vs. transmission in Nd:YAG operating at $1.064 \mu \mathrm{m}$. The line represents the least squares fit to the data with intercept at the origin, according to (3.20).

\subsection{Wedge in the Sample}

When dealing with materials of significant refractive index (greater than $\sim 2$ ), Fresnel reflection and refraction effects become significant. For an index of $n=2.4$, for example, the Fresnel reflection at normal incidence is $17 \%$ per face. These issues require revised thinking when it comes to laser cavity design. An important example is the effect of non- 
parallel surfaces of a laser medium on the laser performance. Since anti-reflective coatings were not available for the crystals in this study, Fresnel losses from such sample "wedges" were important to consider. It is well known that for perfectly parallel surfaces the Fresnel reflections are not lost because they are contained by the resonator geometry. For example, a cavity with two plane parallel mirrors will contain reflected rays that are parallel to the resonator axis. A wedge in the sample in this case would divert rays off axis, both by reflection and by refraction inside the material, and these would walk out of the cavity and be totally lost, reducing laser performance. So the question naturally arises, "How much wedge can a given cavity geometry tolerate before rays are lost?" To answer this question, a ray tracing program was developed to model rays inside a cavity when subjected to a wedge in the gain medium. Ray tracing is a simple way to follow the trajectory of a ray which is covered in many textbooks. ${ }^{56}$ This method, however, does not address the phase of the cavity radiation, and therefore cannot tell the complete story. Even if rays are completely contained, loss can still occur if the phase of the reflections does not add coherently. Nonetheless, ray-tracing is a useful tool in the analysis of cavity stability. In the paraxial approximation a ray can be quantified by its angle from the optic axis $\theta$ and its radial distance from the axis $r$, as shown in Figure 3.6 for the case of propagation through space by a distance $d_{12}$.

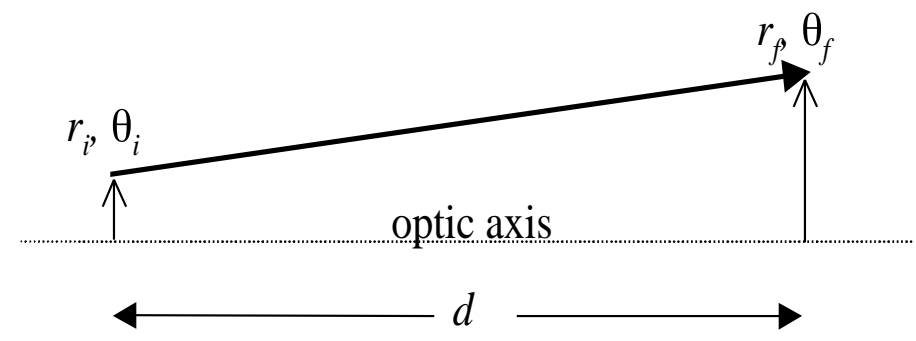

Figure 3.6. A ray is characterized by is distance from the optic axis $r$ and its angle with the axis $\theta$. 
For a given propagation mode, final values of $\theta$ and $r$ are written in terms of the initial values. For the case shown in Figure 3.6, we can write

$$
\begin{aligned}
& r_{f}=r_{i}+d \theta_{i} \\
& \theta_{f}=\theta_{i}
\end{aligned} .
$$

The model needed to address three modes of propagation inside the cavity: (1) propagation through free space a distance $d$, denoted $S(d),(2)$ propagation at height $h$ through a wedged sample of length $l$, index $n$ and wedge angle $\alpha$, denoted $W(\alpha, n, l, h)$ and (3) propagation at a mirror with radius of curvature $\mathrm{R}$, denoted $M(R)$. Two problems need to be addressed. The first is whether the cavity is stable with respect to rays not including Fresnel reflections from the sample. The second is whether Fresnel reflections are lost when the cavity is in a configuration stable with respect to the original ray. Let us examine the first case and look only at an original ray. A round-trip through a cavity, shown in Figure 3.7 , can be represented as $S\left(d_{1}\right) \cdot W(\alpha, n, l, h) \cdot S\left(d_{2}\right) \cdot M\left(R_{2}\right)$ $S\left(d_{2}\right) \cdot W(\alpha, n, l, h) \cdot S\left(d_{1}\right) \cdot M\left(R_{1}\right)$

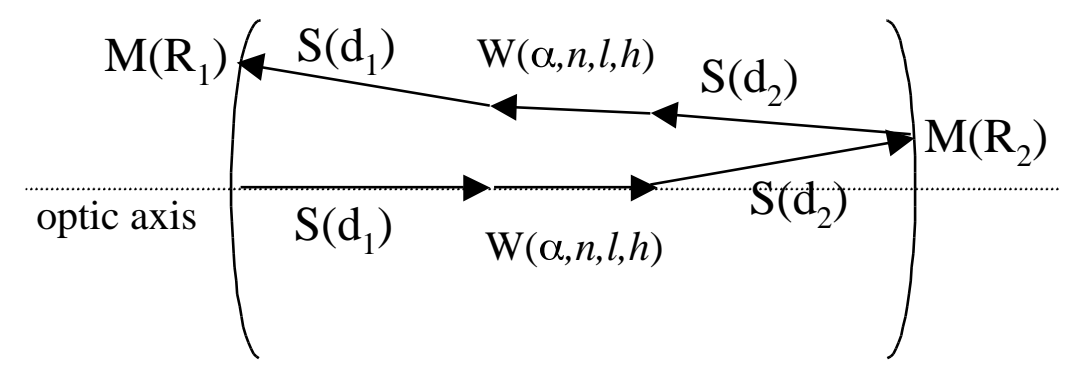

Figure 3.7. Schematic of the propagation modes inside the cavity. The sample has index $n$, wedge angle $\alpha$, and axial length $l$. The optic axis intersects the sample a distance $h$ from the lower edge. $d_{1}$ and $d_{2}$ are the optical distances from the mirror to each sample surface, and the mirrors have reflectivities $R_{1}$ and $R_{2}$, as shown. 
$S(d)$ is given by (3.21). $M(R)$ is given by

$$
\begin{aligned}
& r_{f}=r_{i} \\
& \theta_{f}=\theta_{i}-\frac{2}{R} r_{i} .
\end{aligned}
$$

These formulations are well known. $W(\alpha, n, l, h)$, however, needed to be derived. Figure 3.8 shows the relevant geometry for this derivation.

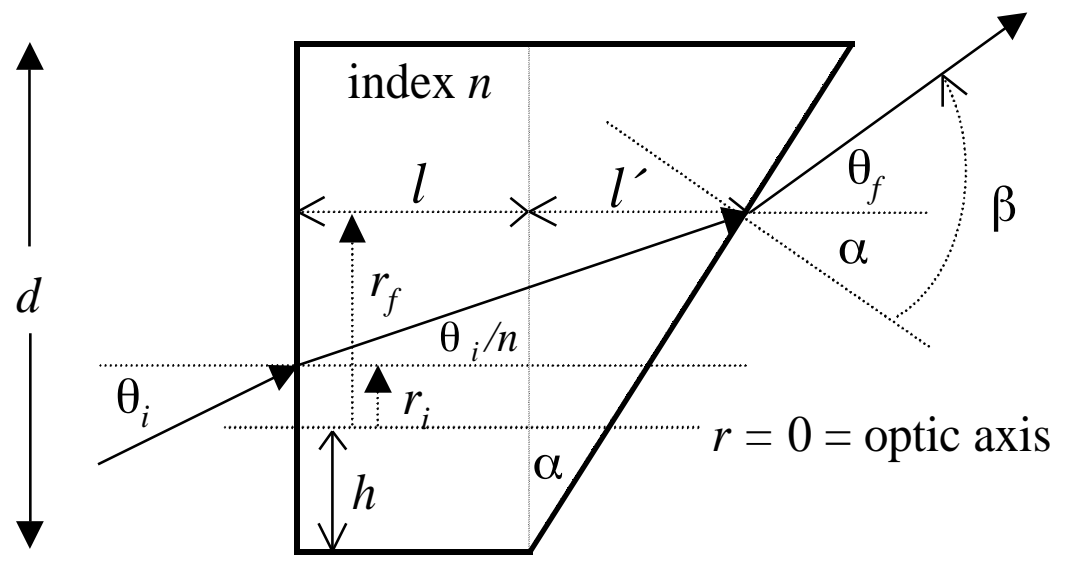

Figure 3.8. Geometry of a ray passing through a wedged sample. The sample wedge angle $\alpha$ is shown exaggerated for clarity.

Using Snell's law and small angle approximations we have

$$
\tan \frac{\theta_{i}}{n} \sqrt{ } \cup \frac{\theta_{i}}{n}=\frac{r_{f}-r_{i}}{l+l^{\prime}}
$$

and

$$
\tan (\alpha) \cup \alpha=\frac{l^{\prime}}{r_{f}+h}
$$

eliminating $l^{\prime}$ from these two equations yields 


$$
r_{f}=\frac{r_{i}+\frac{\theta_{i}}{n}(l+\alpha h)}{1-\frac{\theta_{i}}{n} \alpha} \cup r_{i}+\frac{(l+\alpha h)}{n} \theta_{i}
$$

Similarly we can say $\beta=n\left(\alpha+\theta_{i} / n\right)$ and $\theta_{f}=\beta-\alpha$, so that

$$
\theta_{f}=\theta_{i}+\alpha(n-1)
$$

Equations (3.25) and (3.26) represent $W(\alpha, n, l, h)$. Notice the form of (3.26) does not allow it to be used in simple matrix manipulations, since it is not of the form $\theta_{f}=A r_{i}+B \theta_{i}$. The ray tracing program was encoded into a spreadsheet so that many rays could be traced. Two complete round-trips inside a perfectly confocal resonator are shown in Figure 3.9 for a wedge angle $\alpha=3$ milliradians. Other input parameters are indicated in the figure. The net result is that for a perfectly confocal resonator of length $L$, rays are not lost if the sample has transverse dimension

$$
d>\alpha(n-1) L / 2
$$

and the mirrors have radius

$$
r>\alpha(n-1) R
$$

(Recall $R$ is the radius of curvature of the mirror while $r$ is half the diameter of the mirror). The rays retrace themselves after two complete roundtrips, and are not lost provided the medium and the mirrors are large enough to incorporate them. 


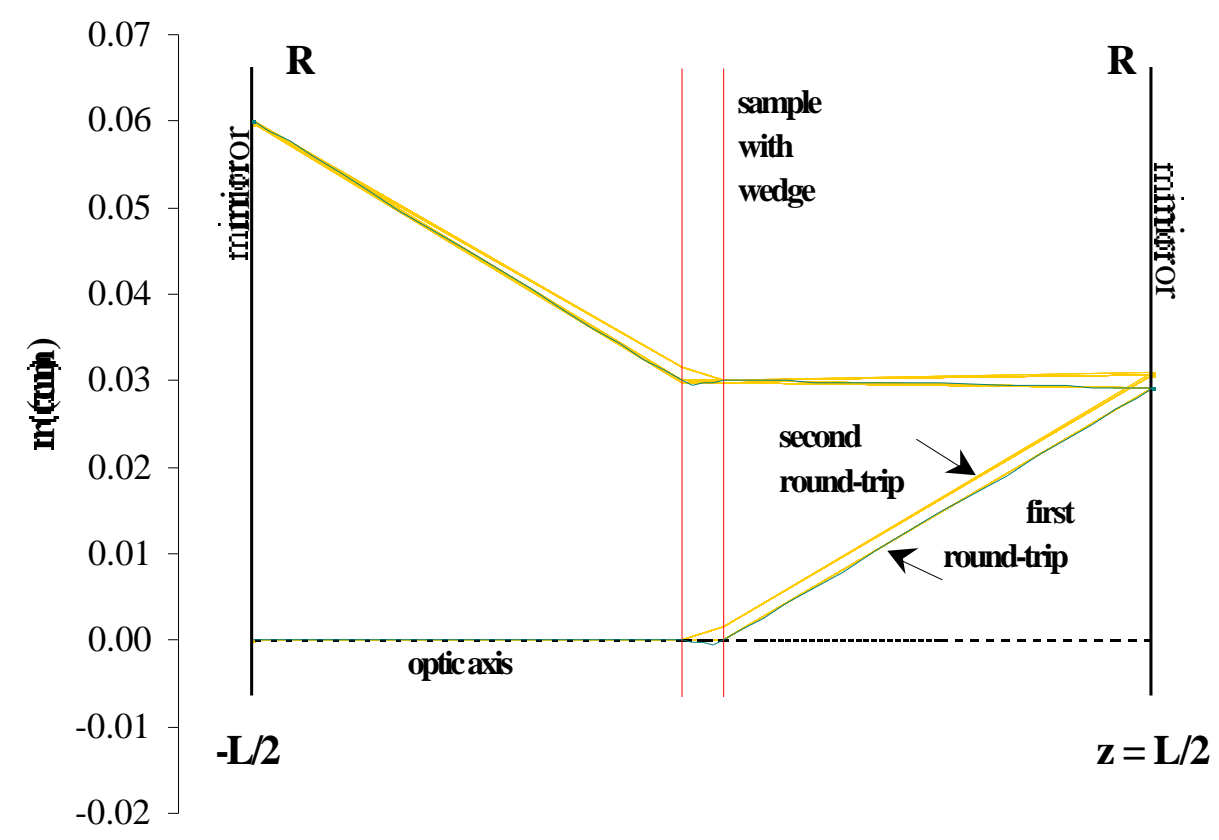

Figure 3.9. Ray-tracing output for two round-trips in a $20-\mathrm{cm}$ confocal cavity $(L=R=20 \mathrm{~cm})$. The wedge angle is $3 \mathrm{mrad}$, the crystal length is $1 \mathrm{~cm}$, and the ray is launched on-axis at $r=0$, starting from the left cavity mirror (of radius $R$ ).

Since in actual experiments a truly perfect confocal cavity is rarely achieved, the effects of non-perfect confocal cavities have also been explored, that is when $L=R \pm \Delta$, where $\Delta$ has a value between 0 and $R$. Figure 3.10 shows ray tracing output similar to Figure 3.9, but for varying values of $\Delta$. The data indicate that the cavity-with-wedge remains stable for $0<L<2 R$, and becomes unstable for $L>2 R$. In fact, the program (as evidenced by the data) indicates that (3.28) remains valid independent of $\boldsymbol{L}$, so that we can write

$$
r_{\min }=\alpha(n-1) R .
$$

The program also indicates (3.27) can be generalized (approximately) for any $L<2 R$ by writing 


$$
d_{\min }=\alpha(n-1)[R-L / 2] \text {. }
$$

Next we need to examine whether Fresnel reflections of the original rays are lost in such a cavity. Tracking each reflection from each surface becomes exceedingly complex. It should suffice to track a single reflection under all possible initial conditions (launching parameters), since if the first order reflection can't escape the cavity, due to the symmetry of the cavity there is no mechanism by which higher order reflections could escape. In fact, since the problem is linear, only the two most extreme initial conditions will provide the necessary information. Accordingly, another program was written to track the first Fresnel reflection from the wedged surface. Fig shows the ray trace of the first reflection from the wedged surface for the same conditions of Figure 3.10. This analysis provides a general conclusion that Fresnel reflections are contained if

$$
d \cup r>2 r_{\min }
$$

where $r_{\min }$ is given by (3.29). Thus the maximum tolerable wedge angle is approximately given by

$$
\alpha_{\text {max }}=\frac{d}{2(n-1) R} .
$$

For values typical of these studies, $d=2-\mathrm{mm}, n=2$, and $R=20-\mathrm{cm}$, we conclude $\alpha_{\max }=$ $5 \mathrm{mrad}$. Typical polishing efforts have produced wedge angles of $3 \mathrm{mrad}$ or less for the crystals used in this study. While this analysis does not include interference effects (i.e. the phase of the cavity photons has not been included), it does indicate laser performance should remain relatively unaffected by a wedged sample (provided (3.32) is satisfied), since Fresnel reflections are not lost and the cavity remains stable. 
To more fully characterize the cavity, future efforts would need address two issues: (1) the phase relationships of the rays and (2) the biaxial nature of crystal. The former would entail modeling the light not simply as 'rays', but as Gaussian beams propagating in the cavity. The latter would presumably behave as an additional 'wedge', either enhancing or reducing the overall wedge effect, depending on the orientation of the physical wedge. The angle for the biaxial 'wedge' would correspond to the walk-off angle between the different rays propagating in the crystal. Ref. [57] covers some of the basics of electromagnetic propagation in anisotropic media. 

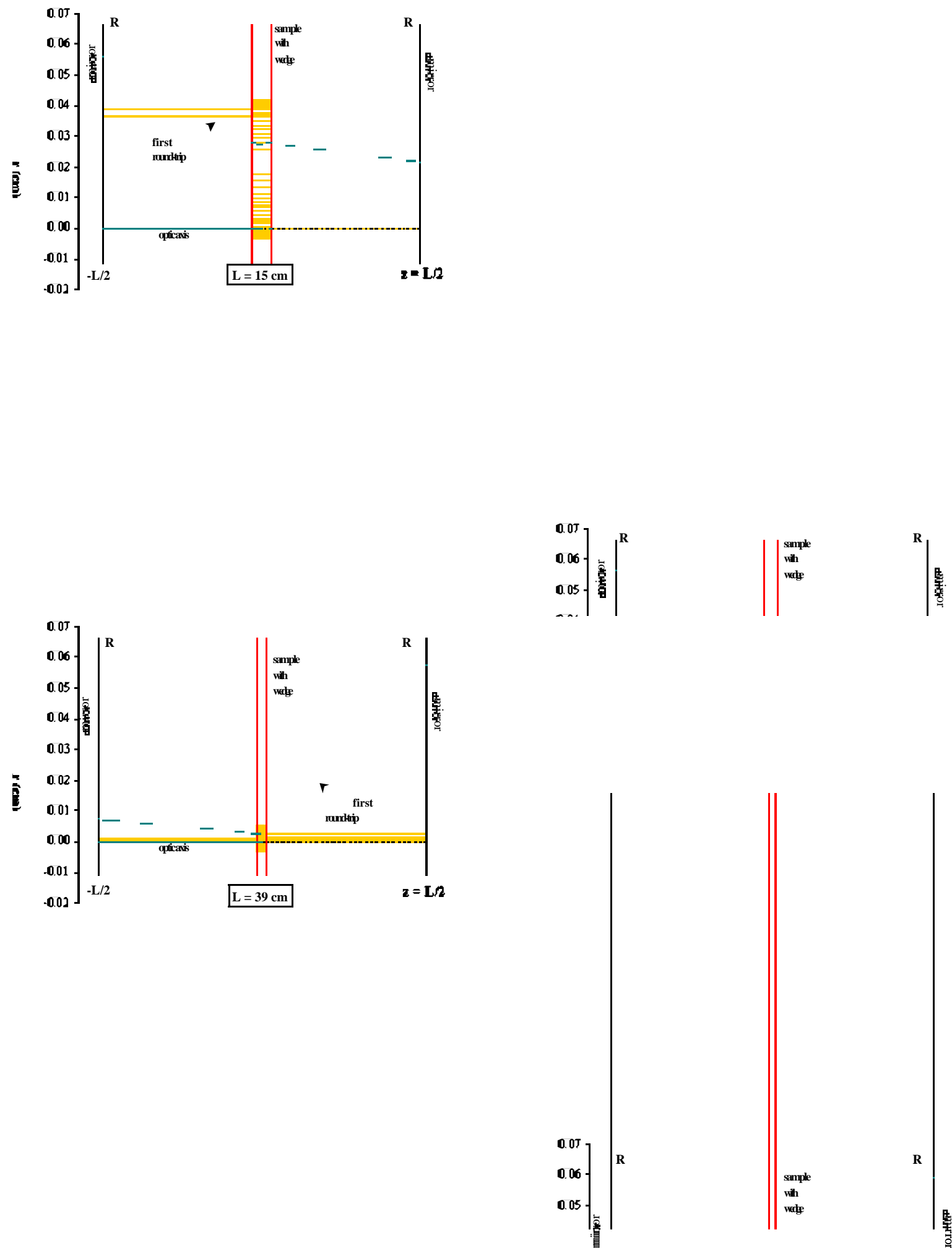

Figure 3.10. Ray tracing output for a symmetric cavity with $R=20 \mathrm{~cm}$ for varying cavity length $\boldsymbol{L}$. The cavity contains a wedged sample $1 \mathrm{~cm}$ in length with wedge angle $\alpha=3 \mathrm{mrad}$. The initial ray is launched on-axis at $\mathbf{r}=\mathbf{0}$, starting at the left mirror. The first round trip is indicated by the darker line. The total output in each case reflects 100 round-trips. The cavity becomes unstable for $L>2 R$. 

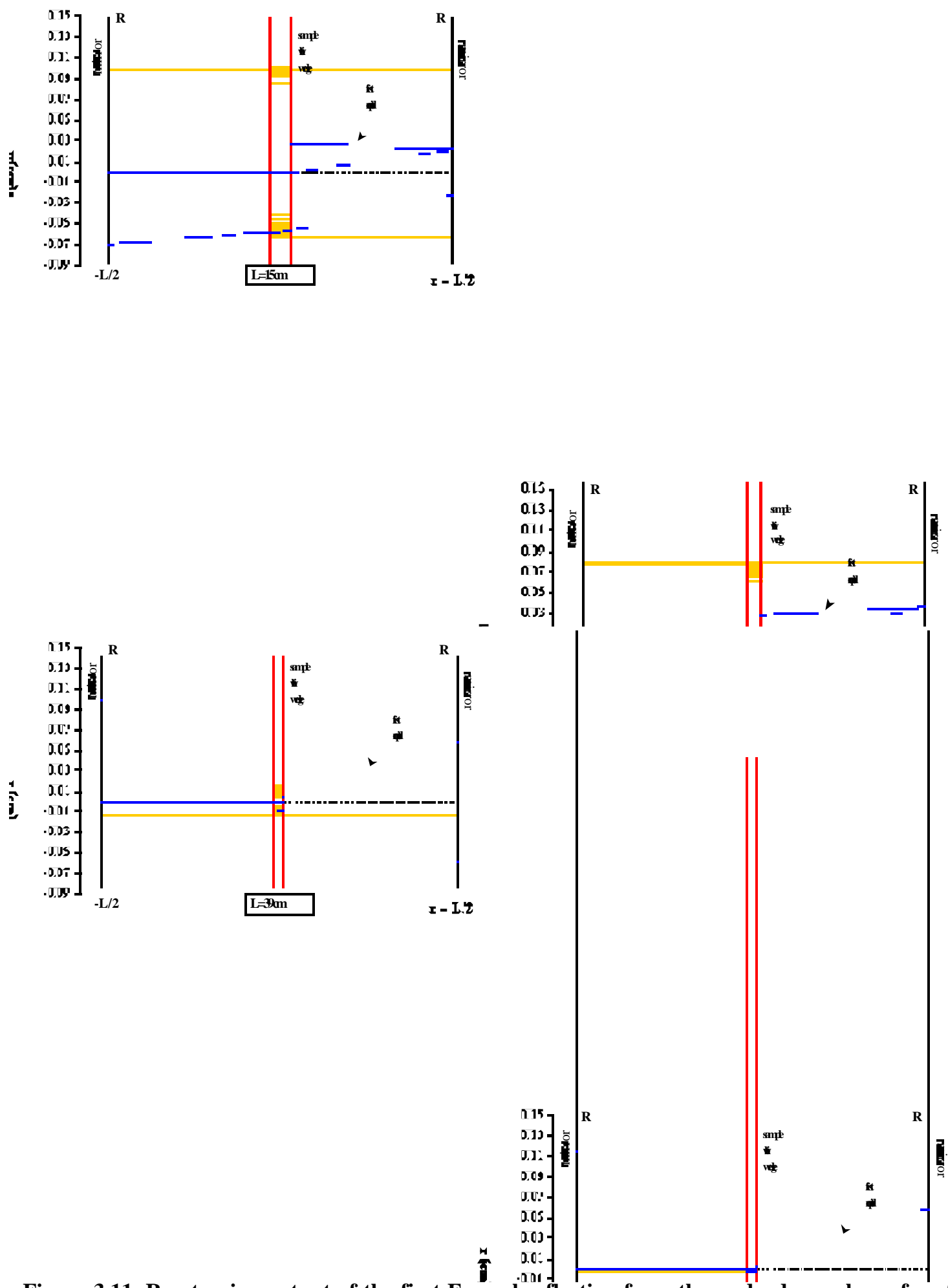

Figure 3.11. Ray tracing output of the first Fresnel reflection from the wedged sample surface for a symmetric cavity with $R=20 \mathrm{~cm}$ for varying cavity length $L$. The cavity contains a wedged sample 1 cm in length with wedge angle $\alpha=3$ mrad. The initial ray is launched on-axis at $r=0$, starting at the left mirror. The first round-trip (including the first Fresnel reflection) is indicated by the darker line. The total output in each case contains 100 round-trips. The on-axis launch represents the most extreme case in terms of radial expansion of the rays. 


\section{Growth and Characterization of $\mathrm{KPb}_{2} \mathrm{Cl}_{5}: \mathrm{Dy}^{3+}$}

In general, chloride materials are known to possess lower phonon energies than oxide or fluoride materials. Experience has shown that chloride materials suitable for rare-earthdoped laser hosts are difficult to find. $\mathrm{LaCl}_{3}$, for example, can be doped with rare-earth ions and grown in large single crystals with high optical quality, but suffers from a high sensitivity to moisture, rendering ambient-condition operation impossible. $\mathrm{PbCl}_{2}$ is known to be stable in ambient conditions and can also be grown in large single crystals with high optical quality, but does not incorporate rare-earth ions effectively into its lattice. In an attempt to find a chloride that combined the rare-earth solubility of $\mathrm{LaCl}_{3}$ and the moisture sensitivity of $\mathrm{PbCl}_{2}$, many chloride crystals were grown at the Institute of Monocrystals, including $\mathrm{BaCl}_{2}, \mathrm{PbCl}_{2}, \mathrm{KPb}_{2} \mathrm{Cl}_{5}, \mathrm{AgCl}$, and $\mathrm{SrCl}_{2}$. In each case,

doping with $\mathrm{Dy}^{3+}$ was attempted. These crystals were known from the literature, but generally their rare-earth solubility and moisture sensitivity were not known. Of these trial efforts, one crystal stood out: $\mathrm{KPb}_{2} \mathrm{Cl}_{5}$.

\subsection{Sample Preparation}

Single crystals of $\mathrm{KPb}_{2} \mathrm{Cl}_{5}$ typically measuring $2.5 \mathrm{~cm}$ in length and 8 - $\mathrm{mm}$ diameter were grown at the Design and Technological Institute for Monocrystals in Novosibirsk, Russia using the Bridgman technique in a two-zone furnace with vertical gradient $20 \mathrm{deg} / \mathrm{cm}$. (The Czochralski method is not used due to the higher volatility of the $\mathrm{KCl}-\mathrm{PbCl}_{2}$ compounds.) Figure 4.1 shows one of the $\mathrm{KPb}_{2} \mathrm{Cl}_{5}: \mathrm{Dy}^{3+}$ samples grown for these experiments. 


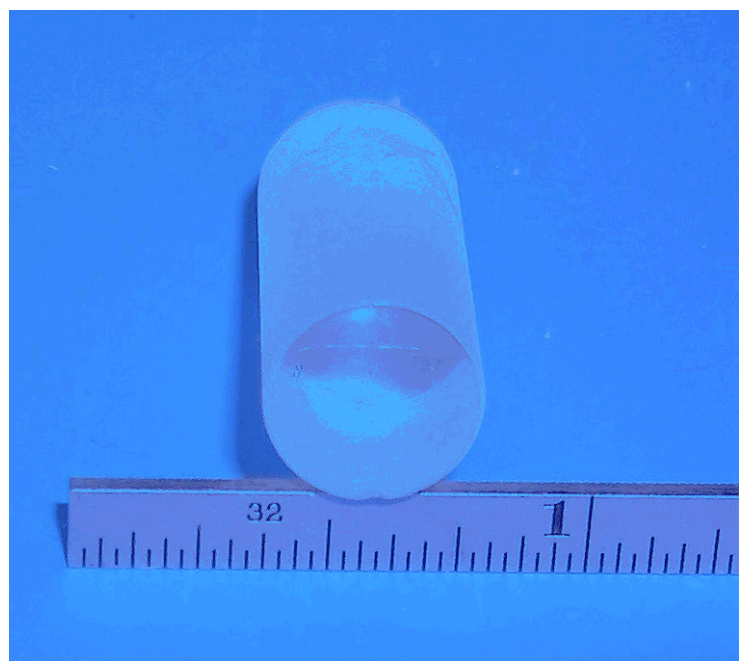

Figure 4.1. The $\mathrm{KPb}_{2} \mathrm{Cl}_{5}: \mathrm{Dy}^{3+}$ crystals were grown by the Bridgman technique in a two-zone furnace.

The $\mathrm{Dy}^{3+}$ ion is believed to dope onto the $\mathrm{Pb}^{2+}$ site, with $\mathrm{K}^{+}$vacancies assumed to be responsible for charge compensation. The $\mathrm{Dy}^{3+}$ content did not exceed 3 mol.\% in the melt, and the segregation coefficient was determined to be $\sim 1$ based on measurements of bulk $\mathrm{Dy}^{3+}$ content with the use of an inductively-coupled plasma mass spectrometer.

\subsection{Crystal Properties}

$\mathrm{KPb}_{2} \mathrm{Cl}_{5}$ is biaxial and belongs to the monoclinic crystal class (space group $\mathrm{P} 2{ }_{1} / \mathrm{c}^{-} \mathrm{C}_{2 \mathrm{~h}}^{5}$ ) with lattice parameters $a=0.8831 \mathrm{~nm}, b=0.7886 \mathrm{~nm}, c=1.2430 \mathrm{~nm}, \alpha=\gamma=90^{\circ}$, and $\beta$ $=90^{\circ} 8^{\prime} .{ }^{14}$ The $\beta$ value near $90^{\circ}$ indicates the crystal is nearly orthorhombic. This crystal belongs to the structural homogeneous family $\mathrm{APb}_{2} \mathrm{X}$, where $\mathrm{A}=\mathrm{K}, \mathrm{Rb}, \mathrm{NH}_{4}$ and $\mathrm{X}=$ $\mathrm{Cl}, \mathrm{Br}^{58}$ The structure of $\mathrm{KPb}_{2} \mathrm{Cl}_{5}$ was studied by Nitsch. ${ }^{14}$ The structure has two symmetry independent lead atoms, referred to as $\mathrm{Pb} 1$ and $\mathrm{Pb} 2$. The coordination of $\mathrm{Pb}^{2+}$ is shown in Figure 4.2. Pb1 is described as a distorted octahedron with one apex doubled. $\mathrm{Pb} 2$ has the same coordination as $\mathrm{Pb}$ in $\mathrm{PbCl}_{2}$, which is described as a distorted 
triagonal prisma with six chlorine atoms at the apexes and three other ones above the centers of the oblong faces.

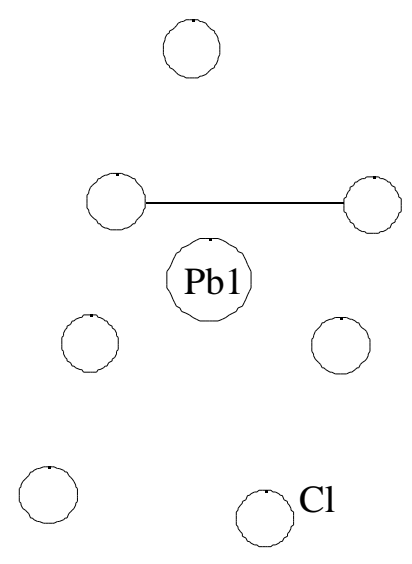

(a)

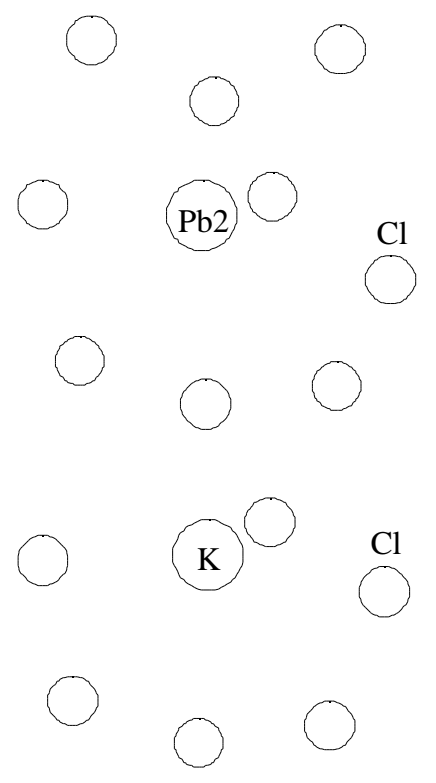

(b)

Figure 4.2. Coordination of lead in $\mathrm{KPb}_{2} \mathrm{Cl}_{5}$. The $\mathrm{KPb}_{2} \mathrm{Cl}_{5}$ structure has two symmetry independent lead ions, $\mathrm{Pb1}$ and $\mathrm{Pb2}$. (a) Pb1 is 7-fold coordinated. (b) Pb2 is 9-fold coordinated. (after Ref. [14])

The planes occupied by $\mathrm{K}^{+}$ions and $\mathrm{Pb}^{2+}$ ions alternate, and are mutually shifted when viewed along an axis.

Gabriel ${ }^{59}$ reports the most recent phase diagram of the $\mathrm{KCl}-\mathrm{PbCl}_{2}$ system, shown in Figure 4.3. $\mathrm{KPb}_{2} \mathrm{Cl}_{5}$ is congruent with melting temperature $434^{\circ} \mathrm{C}$, and has a phase transition at $270^{\circ} \mathrm{C}$. 


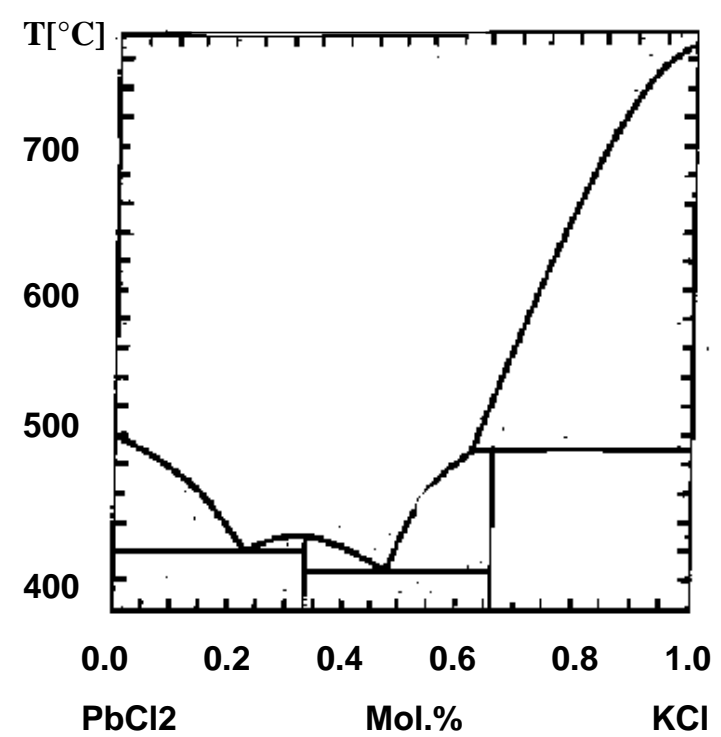

Figure 4.3. Phase diagram of the KCl-PbCl ${ }_{2}$ system. (after Ref. [59])

The maximum phonon energy was determined by A.P. Yelisseyev at the Design and Technological Institute for Monocrystals from Raman scattering spectra in $\mathrm{KPb}_{2} \mathrm{Cl}_{5}$ powder using a Ramanor U-1000 spectrometer $\left(1 \mathrm{~cm}^{-1}\right.$ spectral resolution) with $514 \mathrm{~nm}$ argon laser excitation. The results, shown in Figure 4.4, indicate a maximum phonon energy $h v_{\max }=203 \mathrm{~cm}^{-1}$, measured at the peak of the highest-energy feature.

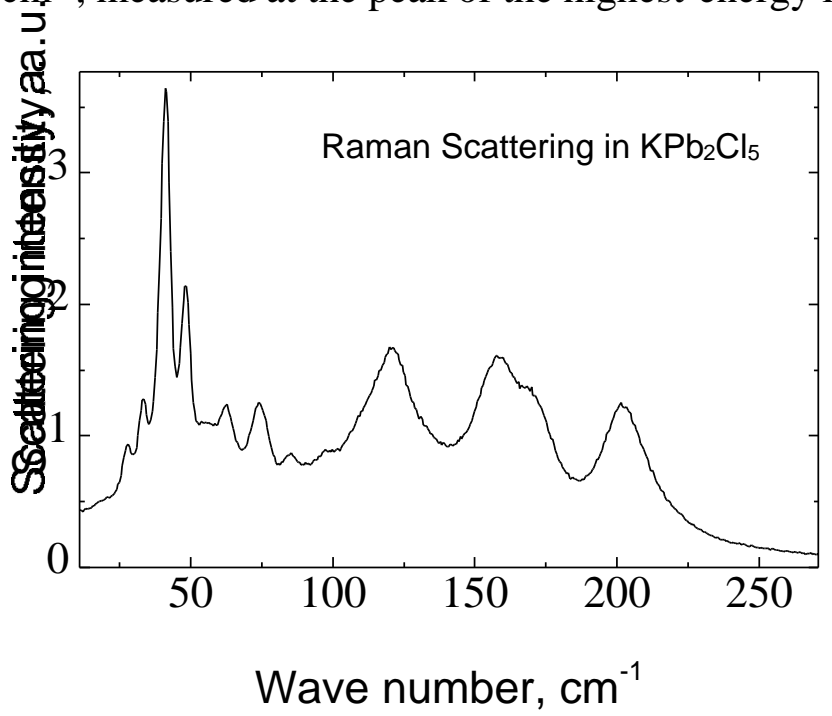

Figure 4.4. Raman phonon spectrum of $\mathrm{KPb}_{2} \mathrm{Cl}_{5}$. The highest feature occurs near $203 \mathrm{~cm}^{-1}$. 
According to factor group analysis ${ }^{60}$ for $\mathrm{KPb}_{2} \mathrm{Cl}_{5}$ related to the $\mathrm{P} 2_{1} / \mathrm{c}_{-} \mathrm{C}^{5}{ }_{2 \mathrm{~h}}$ group with $\mathrm{Z}=4$ and $C_{2}$ axis along $\boldsymbol{b}$ in the unit cell, 96 dispersion branches are expected as $24 A_{g}+24 B_{g}+$ $24 \mathrm{~A}_{\mathrm{u}}+24 \mathrm{~B}_{\mathrm{u}}$. There are $48\left(24 \mathrm{~A}_{\mathrm{g}}+24 \mathrm{~B}_{\mathrm{g}}\right)$ Raman-active and $45\left(23 \mathrm{~A}_{\mathrm{u}}+22 \mathrm{~B}_{\mathrm{u}}\right)$ IR-active modes. As a rule the most intense bands are associated with $\mathrm{A}_{\mathrm{g}}$ symmetry. The superposition of these branches produces the $\sim 15$ bands resolved in the figure. Since the monoclinic $\mathrm{KPb}_{2} \mathrm{Cl}_{5}$ structure contains no molecular groups with specific internal vibrations, the observed spectra can be described in terms of $\mathrm{K}^{+}, \mathrm{Cl}^{-}$, and $\mathrm{Pb}^{2+}$ activity. The features below $75 \mathrm{~cm}^{-1}$ are due to $\mathrm{Pb}^{2+}$ translational modes, while the features between 75 and $100 \mathrm{~cm}^{-1}$ are probably due to $\mathrm{K}^{+}$translational modes. The bands above $100 \mathrm{~cm}^{-1}$ are associated with vibrations involving $\mathrm{Cl}^{-}$, including the band at $203 \mathrm{~cm}^{-1}$ which is believed to be related to vibrations of $\mathrm{Cl}-\mathrm{K}$ bonds. A complete assignment of the spectrum is probably not possible due to the complicated, overlapping nature of the spectral features.

The multiphonon infrared cut-off (shown in Figure 4.5) of $\sim 20 \mu \mathrm{m}$ was measured with a Perkin-Elmer 983 Infrared Spectrophotometer. The absorption feature near 7.2 $\mu \mathrm{m}$ has been identified as a $\mathrm{NH}_{4}{ }^{+}$impurity introduced in purification of the crystals where $\mathrm{NH}_{4} \mathrm{Cl}$ is used as a chlorine agent. The maximum transmission of $\sim 80 \%$ is consistent with Fresnel and scattering losses in the $5 \mathrm{~mm}$ length of sample (See Appendix D). The $\sim 20-\mu \mathrm{m}$ cut-off frequency is consistent with the measured maximum phonon frequency of $200 \mathrm{~cm}^{-1}$ (See Appendix E). 


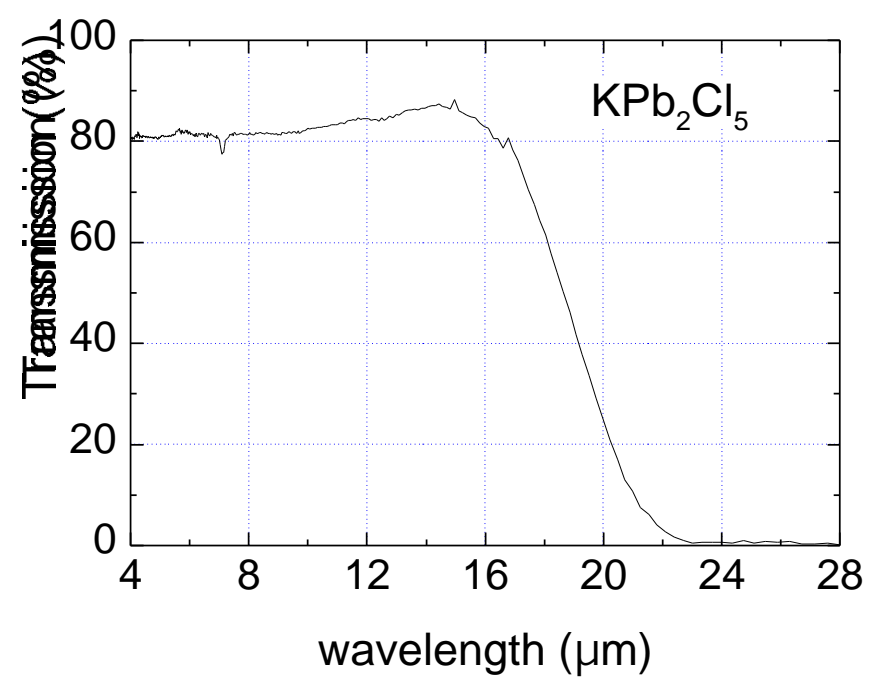

Figure 4.5. Mid-IR transmission spectrum through $5 \mathrm{~mm}$ of $\mathrm{KPb}_{2} \mathrm{Cl}_{5}$.

The refractive index was measured (this study) along two orthogonal axes of an oriented crystal with two orthogonal polarizations of a He-Ne laser using a Metricon ${ }^{\mathrm{TM}}$ prism coupler, which operates by measuring the critical angle necessary for total internal reflection (which is index dependent). By rotating the crystal about each axis, maximum and minimum indices could be determined for each polarization. The maximum index corresponds to $\mathrm{n}_{\mathrm{z}}$, while the minimum corresponds to $\mathrm{n}_{\mathrm{x}}$. By this method, it was found that $\mathrm{n}_{\mathrm{z}}=2.019 \pm 0.001$ and $\mathrm{n}_{\mathrm{x}}=1.982 \pm 0.001$, giving $\Delta \mathrm{n}=0.037 \pm 0.003$ (it appears $\mathrm{n}_{\mathrm{x}} \approx$ $\left.\mathrm{n}_{\mathrm{y}}\right)$.

\subsection{Absorption Spectra}

Absorption spectra at temperatures of $15 \mathrm{~K}$ and $300 \mathrm{~K}$ are shown in Figure 3.1. The cold spectrum shows line narrowing due to the thermal depopulation of the $(2 J+1)$ Starklevels comprising the ground state. Energy levels were assessed based on the peaks of the $15 \mathrm{~K}$ spectrum and are indicated in Figure 3.1. These data will be used in the Judd-Ofelt analysis and the evaluation of multiphonon decay rates below. The Russell-Sauders 
assignments and letter designations of the energy levels are taken after Dieke. ${ }^{15}$ Notice the large relative intensity of the $1.3-\mu \mathrm{m} Z \rightarrow W$ peak, indicating the hypersensitive nature of this transition. Hypersensitivity in a host refers to the situation where a transition is much stronger in the host compared to other hosts (or in aqueous solution). This situation seems to only occur when $\Delta \mathrm{J}= \pm 2$ and $\Delta \mathrm{L}= \pm 2$, which also corresponds to the selection rules for quadrupole radiation. The origin seems to be the inhomogeneity of the dielectric, where the variation of the electric field vector across the rare-earth ion is very much greater than for a homogeneous dielectric, and the intensities of quadrupole transitions are greatly enhanced. ${ }^{61}$

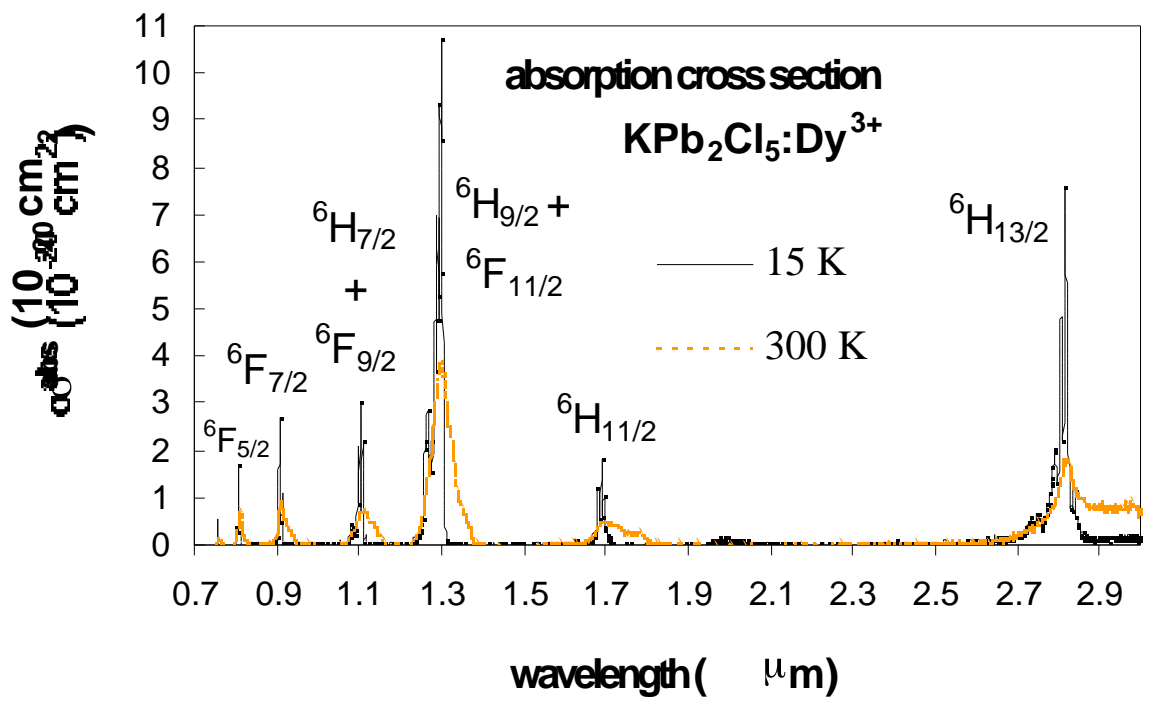

Figure 4.6. Absorption cross section of $\mathrm{KPb}_{2} \mathrm{Cl}_{5}: \mathrm{Dy}^{3+}$ at $15 \mathrm{~K}$ (dark line) and at room temperature (light line), showing line broadening with temperature. Energy level values were determined based on peak-to-peak values of the $15 \mathrm{~K}$ spectrum, and appear in Figure 3.1.

Polarized and unpolarized absorption data were taken with a Perkin Elmer Lambda 9 spectrophotometer. The orthogonal polarization directions were identified at extinction positions between crossed polarizers. As the unpolarized spectra was essentially 
indistinguishable from the polarized spectra, the unpolarized spectra were used in this study. The absorption cross section $\sigma^{a b s}(\lambda)$ was determined from the measured absorption coefficient $\alpha(\lambda)$ by measuring the total $\mathrm{Dy}^{3+}$ concentration $N_{0}$ via the inductively coupled mass spectrometer technique and taking the ratio $\sigma^{a b s}(\lambda)=\alpha(\lambda) / N_{0}$.

A total of five $\mathrm{KPb}_{2} \mathrm{Cl}_{5}$ samples of varying $\mathrm{Dy}^{3+}$ concentration (0.76 - 1.6 mol.\%; see Table 4.1) were used in this study. The samples were grown individually over the course of a few years, each attempt striving for better optical quality. Consequently, the crystals vary in optical quality: samples 1-3 are generally of 'good' optical quality, sample \#4 is 'fair', and sample \#5 is 'poor'. $\mathrm{OH}$ impurities are generally attributed to causing the poor optical quality.

Table 4.1. The $\mathrm{Dy}^{3+}$ concentration of the five samples used in our study. The concentration of sample \#1 was measured directly, and that of samples \#2-\#5 were determined by comparative absorption.

\begin{tabular}{|c|c|c|}
\cline { 2 - 3 } \multicolumn{1}{c|}{} & \multicolumn{2}{c|}{$\mathbf{D y}^{\mathbf{3 +}}$ Concentration, $\mathbf{N}_{\mathbf{~}}$} \\
\hline Sample \# & $\mathbf{1 0}^{\mathbf{2 0}} \mathbf{c m}^{-\mathbf{3}}$ & $\mathbf{\text { mol.\%}}$ \\
\hline 1 & 0.35 & 0.76 \\
\hline 2 & 0.40 & 0.87 \\
\hline 3 & 0.43 & 0.93 \\
\hline 4 & 0.54 & 1.17 \\
\hline 5 & 0.73 & 1.59 \\
\hline
\end{tabular}

The $\mathrm{Dy}^{3+}$ concentration for the four other samples (\#2-\#5) was then established by a least squares fit of the absorption coefficient curves of samples \#2 - \#5 to the absolute cross section curve of sample \#1 from above. Table 4.1 lists the results of this analysis. The estimated error in the data is $\pm 10 \%$. 


\subsection{Judd-Ofelt data}

A Judd-Ofelt analysis of the absorption data was performed according to the developments in Section 2.4. Table 4.2 gives the results of this analysis. The $\mathrm{W} \leftarrow Z$ (1.3$\mu \mathrm{m})$ transition has sometimes been omitted from the analysis in the literature due its hypersensitive nature. ${ }^{61,62}$ Since the RMS error of the fit was lower with the $1.3 \mu \mathrm{m}$ transition excluded $(0.16 \%$ vs. $2.44 \%)$, we have also omitted it from this analysis. The three Judd-Ofelt parameters were determined to be: $\Omega_{2}=5.42 \times 10^{-20} \mathrm{~cm}^{2}, \Omega_{4}=0.99 \times$ $10^{-20} \mathrm{~cm}^{2}, \Omega_{6}=2.96 \times 10^{-20} \mathrm{~cm}^{2}$. These results will later be compared to those measured experimentally. 
Table 4.2. Results of the Judd-Ofelt analysis for $\mathrm{KPb}_{2} \mathrm{Cl}_{5}: \mathrm{Dy}^{3+}$.

\begin{tabular}{|c|c|c|c|c|c|c|c|}
\hline Transition & $\begin{array}{c}\lambda \\
(\mu \mathbf{m})\end{array}$ & $\begin{array}{c}\mathrm{S}^{\mathrm{ED}} \\
\left(10^{-20} \mathrm{~cm}^{2}\right)\end{array}$ & $\begin{array}{c}\mathrm{S}^{\mathrm{MD}} \\
\left(10^{-20} \mathrm{~cm}^{2}\right)\end{array}$ & $\begin{array}{l}\mathbf{A}^{\mathrm{ED}} \\
\left(\mathbf{s}^{-1}\right) \\
\end{array}$ & $\begin{array}{l}\mathrm{A}^{\mathrm{MD}} \\
\left(\mathrm{s}^{-1}\right)\end{array}$ & $\beta^{\mathrm{rad}}$ & $\begin{array}{c}\tau_{\mathrm{rad}} \\
(\mathbf{m s}) \\
\end{array}$ \\
\hline${ }^{{ }^{6} \mathrm{H}_{13 / 2}} \rightarrow{ }^{6} \mathrm{H}_{15 / 2}$ & 2.82 & 3.55 & 1.02 & 65.7 & 18.9 & 1.000 & 11.8 \\
\hline${ }^{6} \mathrm{H}_{11 / 2} \rightarrow{ }^{6} \mathrm{H}_{13 / 2}$ & 4.23 & 1.88 & 1.49 & 12.0 & 9.5 & 0.091 & \multirow{2}{*}{4.24} \\
\hline$\rightarrow{ }^{6} \mathrm{H}_{15 / 2}$ & 1.69 & 2.15 & 0.00 & 214.3 & 0.0 & 0.909 & \\
\hline${ }^{6} \mathrm{H}_{9 / 2} \rightarrow{ }^{6} \mathrm{H}_{11 / 2}$ & 5.57 & 1.97 & 1.64 & 6.6 & 5.5 & 0.052 & \multirow{3}{*}{4.29} \\
\hline$\rightarrow{ }^{6} \mathrm{H}_{13 / 2}$ & 2.40 & 1.43 & 0.00 & 59.5 & 0.0 & 0.255 & \\
\hline$\rightarrow{ }^{6} \mathrm{H}_{15 / 2}$ & 1.30 & 0.61 & 0.00 & 161.4 & 0.0 & 0.693 & \\
\hline${ }^{6} \mathbf{F}_{11 / 2} \rightarrow{ }^{6} \mathbf{H}_{9 / 2}$ & - & 1.48 & 0.05 & 0.0 & 0.0 & 0.000 & \multirow{4}{*}{0.67} \\
\hline$\rightarrow{ }^{6} \mathrm{H}_{11 / 2}$ & 5.57 & 2.52 & 0.03 & 7.0 & 0.1 & 0.005 & \\
\hline$\rightarrow{ }^{6} \mathrm{H}_{13 / 2}$ & 2.40 & 3.81 & 0.00 & 132.3 & 0.0 & 0.089 & \\
\hline$\rightarrow{ }^{6} \mathrm{H}_{15 / 2}$ & 1.30 & 6.13 & 0.00 & 1354.3 & 0.0 & 0.907 & \\
\hline${ }^{6} \mathrm{H}_{7 / 2} \rightarrow{ }^{6} \mathrm{~F}_{11 / 2}$ & 7.60 & 0.44 & 0.00 & 0.7 & 0.0 & 0.004 & \multirow{5}{*}{5.08} \\
\hline$\rightarrow^{6} \mathrm{H}_{9 / 2}$ & 7.60 & 1.79 & 1.50 & 2.9 & 2.5 & 0.028 & \\
\hline$\rightarrow{ }^{6} \mathrm{H}_{11 / 2}$ & 3.21 & 1.21 & 0.00 & 26.5 & 0.0 & 0.134 & \\
\hline$\rightarrow{ }^{6} \mathrm{H}_{13 / 2}$ & 1.83 & 0.83 & 0.00 & 98.4 & 0.0 & 0.500 & \\
\hline$\rightarrow{ }^{6} \mathrm{H}_{15 / 2}$ & 1.11 & 0.12 & 0.00 & 65.7 & 0.0 & 0.334 & \\
\hline${ }^{6} \mathbf{F}_{9 / 2} \rightarrow{ }^{6} \mathbf{H}_{7 / 2}$ & - & 1.34 & 0.00 & 0.0 & 0.0 & 0.000 & \multirow{6}{*}{0.65} \\
\hline$\rightarrow{ }^{6} \mathrm{~F}_{11 / 2}$ & 7.60 & 0.14 & 0.54 & 0.2 & 0.7 & 0.001 & \\
\hline$\rightarrow^{6} \mathrm{H}_{9 / 2}$ & 7.60 & 1.04 & 0.00 & 1.4 & 0.0 & 0.001 & \\
\hline$\rightarrow{ }^{6} \mathrm{H}_{11 / 2}$ & 3.21 & 1.69 & 0.01 & 29.4 & 0.3 & 0.019 & \\
\hline$\rightarrow{ }^{6} \mathrm{H}_{13 / 2}$ & 1.83 & 4.51 & 0.00 & 428.3 & 0.0 & 0.277 & \\
\hline$\rightarrow{ }^{6} \mathrm{H}_{15 / 2}$ & 1.11 & 2.55 & 0.00 & 1086.8 & 0.0 & 0.703 & \\
\hline${ }^{6} \mathbf{H}_{5 / 2} \rightarrow{ }^{6} \mathbf{F}_{9 / 2}$ & 10.1 & 0.96 & 0.00 & 0.9 & 0.0 & 0.007 & \multirow{7}{*}{7.64} \\
\hline$\rightarrow{ }^{6} \mathrm{H}_{7 / 2}$ & 10.1 & 1.52 & 0.95 & 1.4 & 0.9 & 0.018 & \\
\hline$\rightarrow{ }^{6} \mathrm{~F}_{11 / 2}$ & 4.33 & 0.06 & 0.00 & 0.7 & 0.0 & 0.005 & \\
\hline$\rightarrow{ }^{6} \mathrm{H}_{9 / 2}$ & 4.33 & 1.13 & 0.00 & 13.5 & 0.0 & 0.103 & \\
\hline$\rightarrow{ }^{6} \mathrm{H}_{11 / 2}$ & 2.44 & 0.85 & 0.00 & 57.1 & 0.0 & 0.436 & \\
\hline$\rightarrow{ }^{6} \mathrm{H}_{13 / 2}$ & 1.55 & 0.18 & 0.00 & 47.1 & 0.0 & 0.360 & \\
\hline$\rightarrow{ }^{6} \mathrm{H}_{15 / 2}$ & 1.00 & 0.01 & 0.00 & 9.2 & 0.0 & 0.070 & \\
\hline
\end{tabular}

\subsection{Decay rates and Energy Transfer}

Decay intensities for the $\mathrm{W}$ level of the five $\mathrm{KPb}_{2} \mathrm{Cl}_{5}: \mathrm{Dy}^{3+}$ samples (see Table 4.1), shown in Figure 4.7, display an approximately single-exponential transient and indicate the total decay rates increase with concentration. The hypothesized origin of this concentration-dependence is the phonon-assisted cross-relaxation process depicted in Figure 3.1 (page 3.1-64), whereby an ion in the ground level interacts with a second ion 
in the $\mathrm{W}$ level so that both ions end up in the $\mathrm{Y}$ level. This process must be phonon assisted since the energy of the final state is $600 \mathrm{~cm}^{-1}$ below the initial state (exothermic). Lifetimes were determined by a least-squares fit according to (2.56).

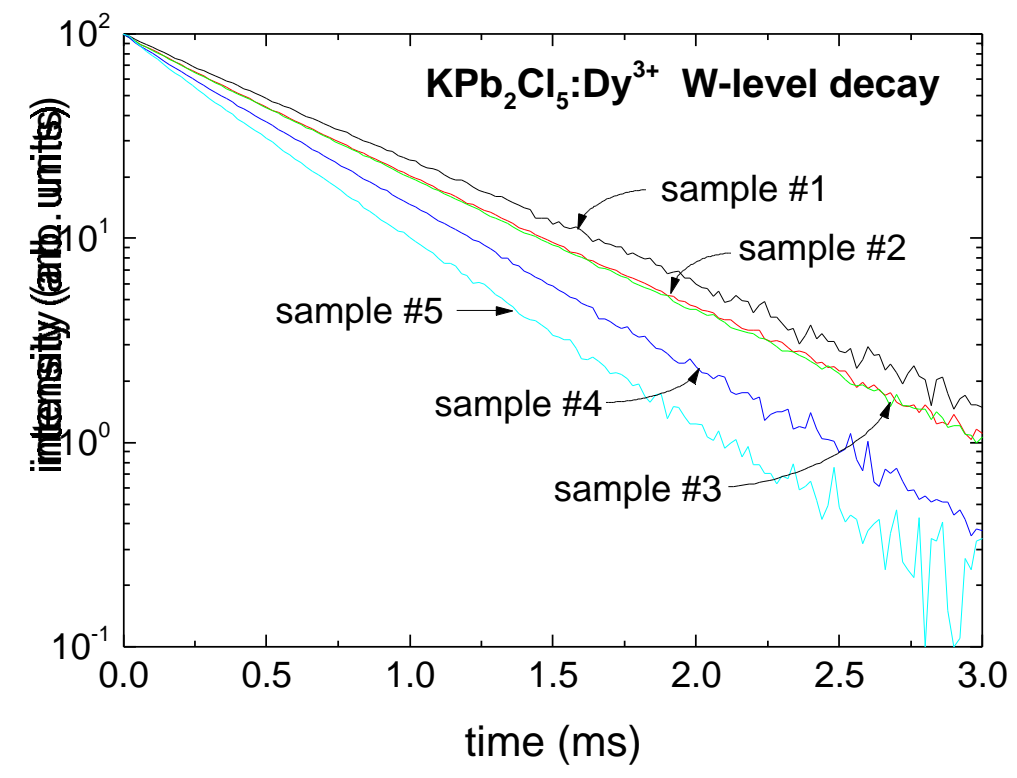

Figure 4.7. Temporal decay of the $\mathrm{W}$ level upon $1.3 \mu \mathrm{m}$ excitation. The decay rate increases as the sample concentration increases, indicative of concentration-dependent cross-relaxation. Notice the decay is largely single exponential for each sample.

As discussed in Section 2.3 the nature of this concentration dependent decay is governed by the relative magnitudes of $R_{D D}$ and $R_{D A}$. Figure 4.8 shows the D-D and D-A cross sections relevant to the cross-relaxation process. These cross sections were calculated from the $\mathrm{F}$ ? chtbauer-Ladenburg relation (2.20) using the Judd-Ofelt data obtained in the previous section. The assistance of phonons in the D-A energy-transfer model was incorporated by using the exponential relation (2.57) with $\bar{N}=3$ and $h v_{\max }=203^{\mathrm{cm}-1}$. An estimate of $\alpha_{s}=0.014 \mathrm{~cm}$ was obtained. These exponential tails are shown appended to the electronic spectra in Figure 4.8b. Using equations (2.44) and (2.45), we estimate $\mathrm{R}_{\mathrm{DD}}$ $=19.4$ (1) and $\mathrm{R}_{\mathrm{DA}}=8.37$ (1). 


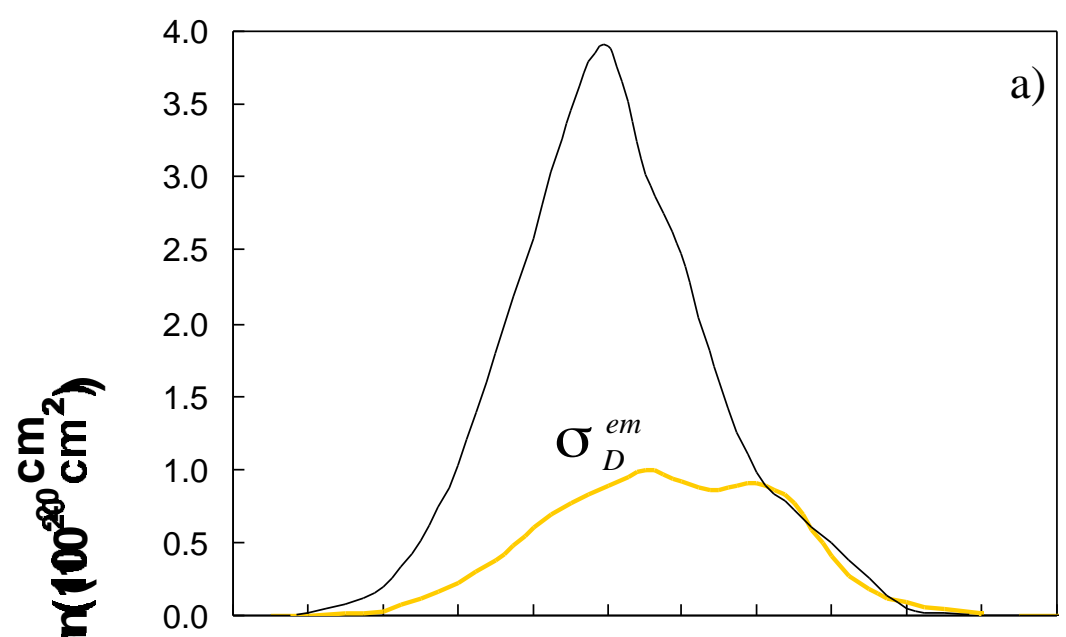

1.201 .221 .241 .261 .281 .301 .321 .341 .361 .381 .401 .42

$\frac{8}{8} 8$

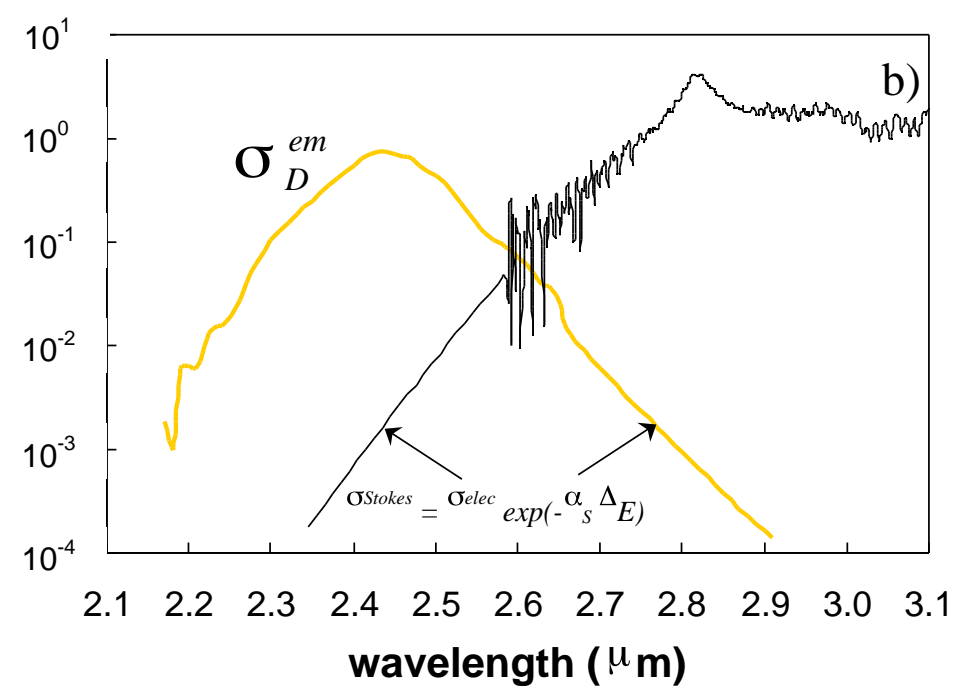

Figure 4.8. The overlap of a) two donor ions and b) a donor and an acceptor ion. The relatively large overlap of the donor ion absorption and emission suggests the hopping mechanism is likely to occur. The latter figure (note the log scale) includes phonon assistance via the multiphonon Stokes sideband contributions to the cross sections.

Thus we are in the regime $R_{D D}>>R_{D A}$, where the hopping model is assumed to be valid.

Since the donor and acceptor are the same species in this case, the cross relation process is characterized by a cross relaxation coefficient $k^{c}$ given by

$$
W^{c}=k^{c} N_{0}^{2}
$$


where here $k^{c}$ is the hopping model value given by (2.55) as

$$
k^{c}=\left[\pi(2 \pi / 3)^{5 / 2}\right] R_{D A}{ }^{3} R_{D D}^{3} A^{\text {rad }} .
$$

The cross relaxation coefficient is computed to be $k^{c}=0.79 \times 10^{-37} \mathrm{~cm}^{6} \mathrm{~s}^{-1}$. While the error bars are difficult to determine for this calculation, they are probably quite large due to the approximate methods of the calculation. Both the hopping model and multiphonon tail model are probably not fully adequate descriptions of the actual energy transfer process in this case, but serve only as adequate approximations. In fact, the actual transfer mechanism may be somewhere between the regimes described by the hopping and diffusion model. As an upper bound to the error bars, it is notable that the value of $k^{c}$ computed using the diffusion model $(2.53)$ is $2.9 \times 10^{-37} \mathrm{~cm}^{6} \mathrm{~s}^{-1}$, indicating the error in the calculation could be as high as a factor of 2 or 3 .

The cross-relaxation coefficient can also be determined experimentally. The total decay rates for the $\mathrm{W}$ level are plotted against the square of the $\mathrm{Dy}^{3+}$ concentration in Fig, along with the fit according to (2.47)and (4.1): $W=W^{0}+k_{c} N_{0}^{2}$.

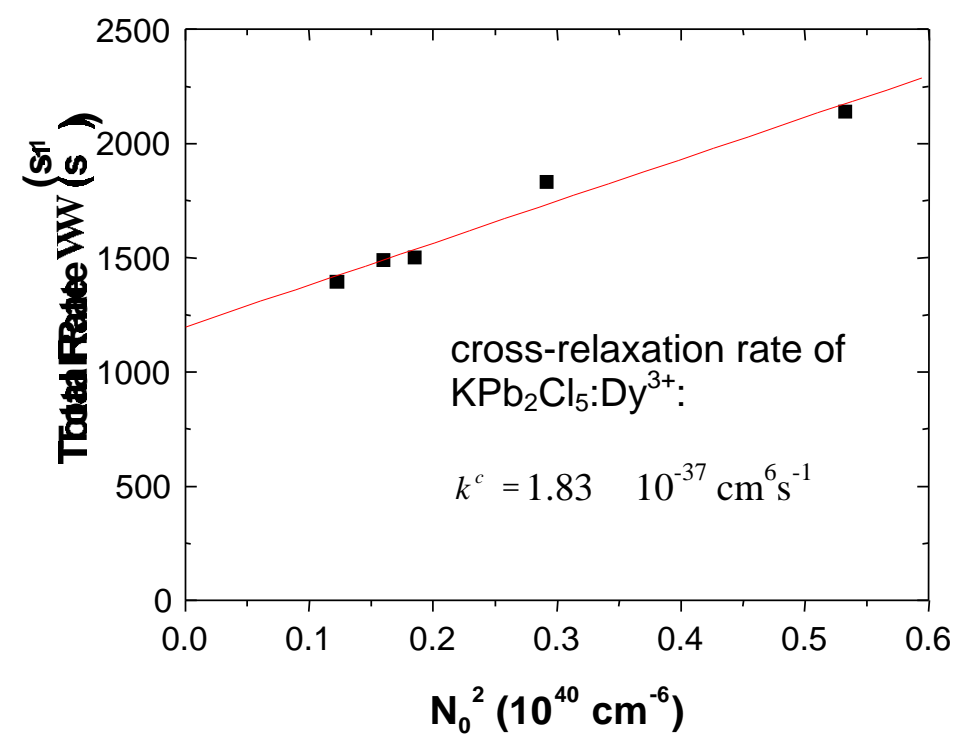


Figure 4.9. The total decay rate for the $W$ level $(W)$ is plotted against the square of the $\mathrm{Dy}^{3+}$ concentration $\left(\mathrm{N}_{\mathbf{0}}{ }^{2}\right)$. Also shown is the fit $\mathbf{W}=\mathbf{W}^{0}+k^{c} \mathbf{N}_{0}^{2}$, where $k^{c}$ is the cross-relaxation coefficient. The slope implies $k^{c}=1.83 \times 10^{-37} \mathrm{~cm}^{6} \mathrm{~s}^{-1}$

Thus the slope of the straight-line fit yields the cross-relaxation coefficient $k^{c}$ while the intercept yields the intrinsic decay rate $W^{0}$. A value of $k^{c}=(1.83 \pm .20) \times 10^{-37} \mathrm{~cm}^{6} \mathrm{~s}^{-1}$ and $W^{0}=1197 \pm 59 \mathrm{~s}^{-1}$ is obtained. This result is in reasonable agreement with the hopping model, establishing the credibility of the cross-relaxation pathway. Based on a similar analysis, it was concluded that there are no appreciable concentration-dependent processes occuring from the $A$ and $X$ levels.

\subsection{Emission Data}

The branching ratios and radiative lifetimes found in Section 4.4 can be used to calculate the emission cross sections of the $\mathrm{KPb}_{2} \mathrm{Cl}_{5}: \mathrm{Dy}^{3+}$ transitions via (2.20). Spectrally calibrated emission spectra were taken with a computer controlled 1-meter scanning monochromator. Sample \#1 was directly excited by a $\mathrm{CW}$ source and a reference chopper was placed at the entrance slit of the monochromator to modulate the emission signal at $77 \mathrm{~Hz}$. The W level was pumped with a $1.319 \mu \mathrm{m}, 100 \mathrm{~mW}$ Nd:YAG laser (manufactured by Amoco) where the absorption coefficient $\alpha \approx 0.9 \mathrm{~cm}^{-1}$, while the $A$ level was pumped with $500 \mathrm{~mW}$ of $1.064 \mu \mathrm{m}$ light from a Nd:YAG laser (manufactured by Lightwave Electronics) where the absorption coefficient $\alpha \approx 0.01 \mathrm{~cm}^{-1}$. Dichroic filters, an InSb detector (77 K), and a SRS lock-in amplifier were used to isolate, detect, and process the signals. For spectra with emission wavelengths below $3.5 \mu \mathrm{m}$, a $2.0 \mu \mathrm{m}$ blazed $300 \mathrm{gr} / \mathrm{mm}$ grating was used, while a $4.0 \mu \mathrm{m}$ blazed, $150 \mathrm{gr} / \mathrm{mm}$ grating was used 
for spectra including wavelengths greater than $3.5 \mu \mathrm{m}$. Individual scans were obtained for wavelengths in the range 1.2- $2.6 \mu \mathrm{m}$ (via A level pumping), $1.7-3.5 \mu \mathrm{m}$, and 2.6-5.5 $\mu \mathrm{m}$ (both via $\mathrm{W}$ level pumping). The 1.2-2.6 $\mu \mathrm{m}$ run with $\mathrm{A}$ level pumping was used to obtain the lineshape of the $1.3-\mu \mathrm{m}$ emission since this data was too difficult to obtain in the presence of the $1.3-\mu \mathrm{m}(\mathrm{X}$ level $)$ pump signal. Emission data was limited to $\lambda<5.5$ $\mu \mathrm{m}$ by the cutoff of the InSb detector. The emission cross section for each transition (as a function of wavelength) was determined separately and then manually combined to form the plot shown in Figure 4.10. Note this data has not taken cross relaxation (see Section 2.3) into account. Such data is included in Section 4.7.

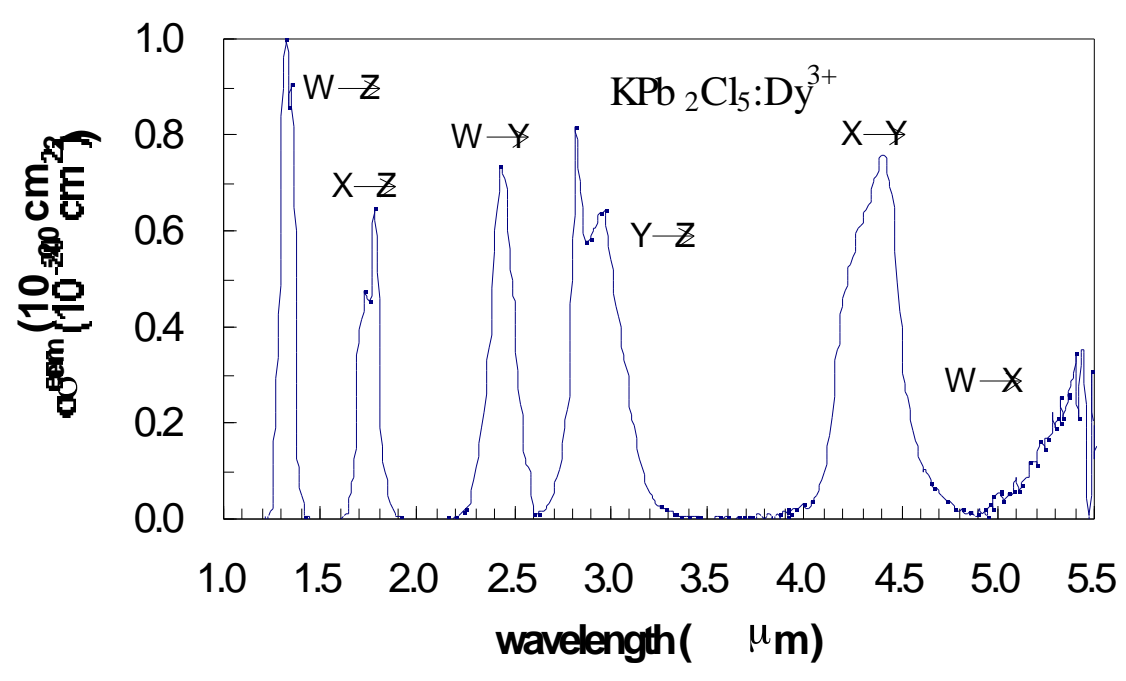

Figure 4.10. Emission cross section of $\mathrm{KPb}_{2} \mathrm{Cl}_{5}: \mathrm{Dy}^{3+}$ calculated with (2.20) using Judd-Ofelt derived radiative lifetimes and branching ratios. Each band was calculated separately and combined manually in the figure for comparison.

The spectral calibration of the raw intensity data was performed with a blackbody source (Electro-Optical Industries model WS142) operating at $1013 \mathrm{~K}$, where the peak emission 
wavelength is $2.9 \mu \mathrm{m}$. In this way, the raw emission intensity could be converted to units of arbitrary $\mathrm{W} / \mathrm{nm}$.

\subsection{Quantum Efficiencies}

As the accuracy of the data found in the previous section is dependent upon the JuddOfelt derived radiative lifetime and branching ratio, it is important to check these values experimentally. Section 3.1 developed a method for determining the radiative quantum efficiencies, lifetimes, and branching ratios for $\mathrm{KPb}_{2} \mathrm{Cl}_{5}: \mathrm{Dy}^{3+}$. This method relies on calibrated emission spectra similar to that found in the previous section, but pumped at specific wavelengths. The experimental set-up used to obtain these spectra was the same as described there. Note the reference chopper needed to modulate the emission signal (rather than the pump beam) in order to avoid calibration of the lock-in amplifier (See Appendix F). Spectra were obtained by pumping the $A, W$, and $X$ levels. For spectra with emission wavelengths below $3.5 \mu \mathrm{m}$, a $2.0 \mu \mathrm{m}$ blazed $300 \mathrm{gr} / \mathrm{mm}$ grating was used, while a $4.0 \mu \mathrm{m}$ blazed, $150 \mathrm{gr} / \mathrm{mm}$ grating was used for spectra including wavelengths greater than $3.5 \mu \mathrm{m}$. Individual scans were obtained for wavelengths in the range 1.2- 2.6 $\mu \mathrm{m}$ (via A level pumping), $1.7-3.5 \mu \mathrm{m}$, and 2.6-5.5 $\mu \mathrm{m}$ (both via $\mathrm{W}$ level pumping). These individual curves were then overlapped and scaled via a least squares fitting of the amplitudes of the appropriate overlapping regions to form the continuous curve shown in Figure 4.11. Emission data was limited to $\lambda<5.5 \mu \mathrm{m}$ by the cutoff of the InSb detector. Level $\mathrm{X}$ was pumped with $\mathrm{a} \sim 1 \mathrm{~W} \mathrm{CW}$ InGaAsP-InP diode source at $1.7 \mu \mathrm{m}$ developed here at LLNL. ${ }^{63}$ The data shown in Figure $4.11 \mathrm{~b}$ was collected in a single run with the $4.0 \mu \mathrm{m}$ blazed grating and InSb detector. The branching ratio of the $4.3 \mu \mathrm{m}$ emission was taken from the W-level-pumped data. A-level-pumped data is shown in Figure 
4.11c. However, since only the emission at $1.1 \mu \mathrm{m}(A \rightarrow Z)$ was discernible [the emissions at 1.8 and $3.1 \mu \mathrm{m}(A \rightarrow Y$ and $A \rightarrow X)$ could not be resolved due to the much stronger emissions at 1.7 and $2.9 \mu \mathrm{m}(X \rightarrow Z$ and $Y \rightarrow Z)]$, this data was generated by pumping the ${ }^{6} \mathrm{~F}_{7 / 2}$ level at $0.9 \mu \mathrm{m}$ with AlGaAs diodes and collected using the InSb detector with a 600 $\mathrm{g} / \mathrm{mm}$ grating blazed at $1 \mu \mathrm{m}$. 


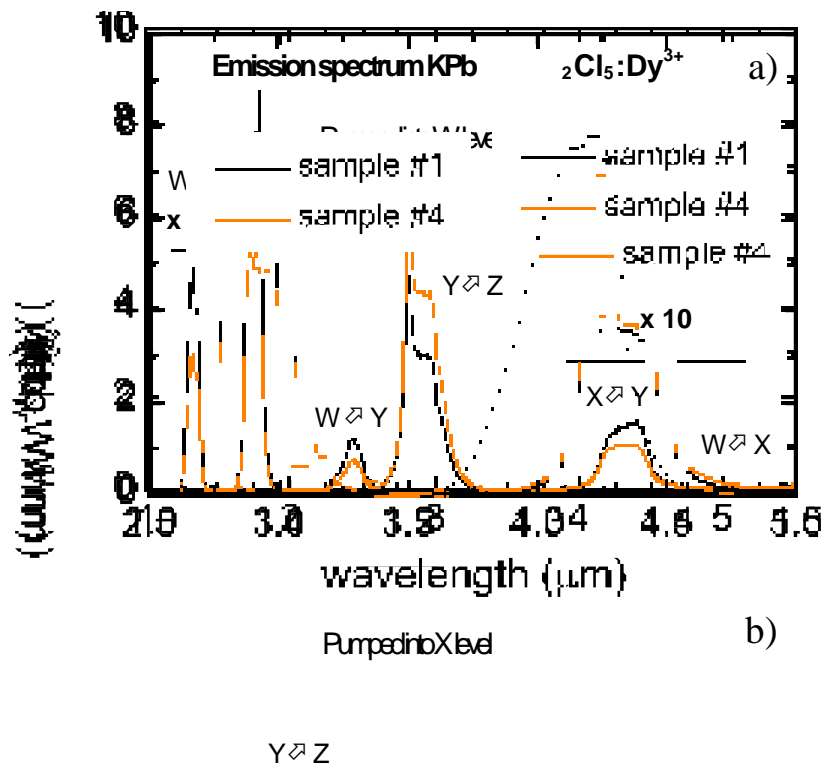

$X \gg Y$

PumpedinbAbed

c)

$W \gg Z$

$A \curvearrowright Z$

$\mathbf{x} 10$

Figure 4.11. Emission data used to determine branching ratios $\beta_{\mathrm{J}}^{\mathrm{rad}}$ and fluorescence ratios ${ }^{P} \phi_{J / K}$ for samples \#1 and \#4. The scales have been adjusted to conserve the total emission rates for each sample. a) Emission spectrum ${ }^{\mathrm{W}} \mathrm{I}(\lambda)$ obtained by direct excitation of the $\mathrm{W}$ level. Note the scale change near $1.3 \mu \mathrm{m}$ and beyond $4 \mu \mathrm{m}$. The transfer of population from the $W$ level to the $Y$ level in the higher doped sample (\#4) can be seen by the relative peak heights of the $W \rightarrow Z$ and $Y \rightarrow Z$ transitions. $b$ ) Emission spectrum obtained by direct excitation of the $X$ level. The 1.7 $\mu \mathrm{m}(X \rightarrow Z)$ feature is not shown. Note the $2.9 \mu \mathrm{m}(\mathrm{Y} \rightarrow \mathrm{Z})$ feature is dramatically reduced compared to that of the $W$-level-pumped spectrum in a). c) Emission spectrum obtained by direct excitation of the $A$ level. Only the $A \rightarrow Z$ and $W \rightarrow Z$ transitions are shown. 
The measured branching ratios $\beta_{\mathrm{J}^{\prime}}^{\mathrm{rad}}$ from (3.10) are given in Table 4.3 and the fluorescence ratios ${ }^{\mathrm{P}}{ }_{\mathrm{JJ} / \mathrm{J}}$, from (3.11)-(3.13) are given in Table 4.4. The resulting quantum efficiencies and radiative lifetimes are shown in Table 4.5. For the $Y$ level, it was assumed that $\eta_{\mathrm{Y}}^{M P}=0$, and that the $\eta_{\mathrm{Y}}^{\text {rad }}$ values less than unity from Table 4.5 are due to oxygen impurities in the host (that nonradiatively depopulate the $\mathrm{Y}$ level), which is evident in the sample \#1 (see Table 4.1 on page $4.3-90$ ) absorption spectrum of Figure $4.6(300 \mathrm{~K})$ near $3 \mu \mathrm{m}$. The total branching ratios $b_{\mathrm{JJ}^{\prime}}$ as defined in (3.4) are shown in Table 4.6.

Table 4.3. Measured radiative branching ratios according to (3.10).

\begin{tabular}{|c||c|c|c||c|c|}
\hline Sample \# & $\boldsymbol{\beta}_{\mathrm{wx}}^{\text {rad }}$ & $\boldsymbol{\beta}_{\mathrm{wr}}^{\text {rad }}$ & $\boldsymbol{\beta}_{\mathrm{wz}}^{\mathrm{rad}}$ & $\boldsymbol{\beta}_{\mathrm{xy}}^{\text {rad }}$ & $\boldsymbol{\beta}_{\mathrm{xz}}^{\text {rad }}$ \\
\hline \hline 1 & 0.008 & 0.076 & 0.916 & 0.118 & 0.882 \\
\hline 2 & 0.009 & 0.080 & 0.911 & 0.154 & 0.846 \\
\hline 3 & 0.010 & 0.083 & 0.907 & 0.132 & 0.868 \\
\hline 4 & 0.018 & 0.073 & 0.909 & 0.118 & 0.882 \\
\hline 5 & 0.020 & 0.088 & 0.892 & 0.116 & 0.884 \\
\hline Average & $\mathbf{0 . 0 1 3}$ & $\mathbf{0 . 0 8 0}$ & $\mathbf{0 . 9 0 7}$ & $\mathbf{0 . 1 2 8}$ & $\mathbf{0 . 8 7 2}$ \\
\hline
\end{tabular}

Table 4.4. Experimental values for the fluorescence ratios as defined in (3.11)-(3.13). The superscript refers to the pumping level, while the subscripts refer to the originating levels of the emissions.

\begin{tabular}{|c|c|c|c|c|c|}
\hline Sample \# & $w_{\phi W / Y}$ & $\mathrm{w}_{\phi_{\mathrm{X} / \mathrm{Y}}}$ & $w_{\phi x / w}$ & $\mathrm{x}_{\phi \mathrm{Y} / \mathrm{X}}$ & $\mathbf{A}_{\phi W / A}$ \\
\hline 1 & 1.971 & 0.673 & 0.341 & 0.115 & 115.4 \\
\hline 2 & 1.250 & 0.459 & 0.367 & 0.145 & 86.1 \\
\hline 3 & 1.180 & 0.450 & 0.381 & 0.090 & 98.5 \\
\hline 4 & 0.859 & 0.348 & 0.405 & 0.091 & 105.3 \\
\hline 5 & 0.954 & 0.594 & 0.623 & 0.059 & 83.9 \\
\hline
\end{tabular}


Table 4.5. Radiative, multiphonon, and cross-relaxation quantum efficiencies as determined by the analysis of Section 3.1. Radiative lifetimes were determined as the ratio of the fluorescence lifetime $\left(\tau^{\text {meas }}\right)$ to the radiative quantum efficiency $\left(\eta^{\text {rad }}\right)$.

\begin{tabular}{|c|c|c|c|c|c|c|}
\hline Level & Sample \# & $\eta^{\mathrm{rad}}$ & $\eta^{\mathrm{MP}}$ & $\eta^{\mathbf{c}}$ & $\begin{array}{l}\tau^{\text {meas }} \\
(\mathbf{m s})\end{array}$ & $\begin{array}{c}\tau^{\mathrm{rad}}=\tau^{\text {meas }} / \eta^{\mathrm{rad}} \\
(\mathrm{ms})\end{array}$ \\
\hline \multirow{6}{*}{ A } & 1 & $0.006 \pm 0.002$ & $0.994 \pm 0.002$ & $0.000^{\mathrm{a}}$ & 0.0052 & 0.94 \\
\hline & 2 & $0.007 \pm 0.002$ & $0.993 \pm 0.002$ & $0.000^{\mathrm{a}}$ & 0.0051 & 0.74 \\
\hline & 3 & $0.006 \pm 0.002$ & $0.994 \pm 0.002$ & $0.000^{\mathrm{a}}$ & 0.0049 & 0.82 \\
\hline & 4 & $0.004 \pm 0.002$ & $0.996 \pm 0.002$ & $0.000^{\mathrm{a}}$ & 0.0056 & 1.26 \\
\hline & 5 & $0.004 \pm 0.002$ & $0.996 \pm 0.002$ & $0.000^{\mathrm{a}}$ & 0.0048 & 1.17 \\
\hline & Ave. & $0.005 \pm 0.001$ & $0.995 \pm 0.001$ & 0.000 & 0.0052 & 0.99 \\
\hline \multirow{6}{*}{ W } & 1 & $0.642 \pm 0.022$ & $0.216 \pm 0.014$ & $0.142^{\mathrm{c}} \pm 0.007$ & 0.717 & 1.12 \\
\hline & 2 & $0.593 \pm 0.020$ & $0.210 \pm 0.012$ & $0.197^{\mathrm{C}} \pm 0.010$ & 0.671 & 1.13 \\
\hline & 3 & $0.590 \pm 0.019$ & $0.208 \pm 0.011$ & $0.202^{\mathrm{c}} \pm 0.010$ & 0.667 & 1.13 \\
\hline & 4 & $0.471 \pm 0.020$ & $0.184 \pm 0.011$ & $0.346^{\mathrm{c}} \pm 0.017$ & 0.547 & 1.16 \\
\hline & 5 & $0.344 \pm 0.021$ & $0.216 \pm 0.012$ & $0.441^{\mathrm{c}} \pm 0.022$ & 0.467 & 1.36 \\
\hline & Ave. & c.d. $^{\mathrm{e}}$ & $0.207 \pm 0.005$ & c.d. $^{\mathrm{e}}$ & c.d. ${ }^{\mathrm{e}}$ & 1.18 \\
\hline \multirow{6}{*}{$\mathbf{x}$} & 1 & $0.991 \pm 0.049$ & $0.009 \pm 0.049$ & $0.000^{\mathrm{a}}$ & 5.964 & 6.02 \\
\hline & 2 & $1.010 \pm 0.047$ & $0.000 \pm 0.047$ & $0.000^{\mathrm{a}}$ & 6.394 & 6.39 \\
\hline & 3 & $1.049 \pm 0.048$ & $0.000 \pm 0.048$ & $0.000^{\mathrm{a}}$ & 6.493 & 6.49 \\
\hline & 4 & $0.994 \pm 0.059$ & $0.006 \pm 0.059$ & $0.000^{\mathrm{a}}$ & 6.939 & 6.98 \\
\hline & 5 & $0.962 \pm 0.100$ & $0.038 \pm 0.100$ & $0.000^{\mathrm{a}}$ & 6.565 & 6.83 \\
\hline & Ave. & $1.001 \pm 0.027$ & $0.011 \pm 0.027$ & 0.000 & 6.471 & 6.54 \\
\hline \multirow{6}{*}{$\mathbf{Y}$} & 1 & $0.904^{\mathrm{b}} \pm 0.073$ & $0.000^{\mathrm{a}}$ & $0.000^{\mathrm{a}}$ & 14.62 & 16.2 \\
\hline & 2 & $1.003 \pm 0.082$ & $0.000^{\mathrm{a}}$ & $0.000^{\mathrm{a}}$ & 15.46 & 15.5 \\
\hline & 3 & $1.061 \pm 0.084$ & $0.000^{\mathrm{a}}$ & $0.000^{\mathrm{a}}$ & 15.31 & 15.3 \\
\hline & 4 & $0.731^{\mathrm{b}} \pm 0.071$ & $0.000^{\mathrm{a}}$ & $0.000^{\mathrm{a}}$ & 16.16 & 22.1 \\
\hline & 5 & $0.381^{\mathrm{b}} \pm 0.047$ & $0.000^{\mathrm{a}}$ & $0.000^{\mathrm{a}}$ & 11.40 & 29.9 \\
\hline & Ave. $^{d}$ & $0.989 \pm 0.046$ & 0.000 & $0.032 \pm 0.046$ & 15.13 & 15.7 \\
\hline
\end{tabular}

a) assumed value

b) values less than unity assumed to be due to oxygen impurities in the host

c) determined from measured lifetime data via (3.5)

d) samples \#4 and \#5 have been omitted from the average

e) concentration dependent

Table 4.6. Total branching ratios as defined in (3.4).

\begin{tabular}{|c||c|c|c||c|c|}
\hline Sample \# & $\mathbf{b}_{\mathbf{W X}}$ & $\mathbf{b}_{\mathbf{W Y}}$ & $\mathbf{b}_{\mathbf{W Z}}$ & $\mathbf{b}_{\mathbf{X Y}}$ & $\mathbf{b}_{\mathbf{X Z}}$ \\
\hline \hline 1 & 0.221 & 0.191 & 0.588 & 0.126 & 0.874 \\
\hline 2 & 0.216 & 0.244 & 0.540 & 0.154 & 0.846 \\
\hline 3 & 0.214 & 0.251 & 0.535 & 0.132 & 0.868 \\
\hline 4 & 0.192 & 0.380 & 0.428 & 0.124 & 0.876 \\
\hline 5 & 0.223 & 0.471 & 0.306 & 0.150 & 0.850 \\
\hline Average & $\mathbf{0 . 2 1 3}$ & c.d. $^{*}$ & c.d. $^{*}$ & $\mathbf{0 . 1 3 7}$ & $\mathbf{0 . 8 6 3}$ \\
\hline
\end{tabular}

* concentration dependent 
The justification of the use of macroscopic rate equations in our model is not obvious. Although there is a random variation in the distance between each microscopic donor-acceptor pair, the donor decay are observed to be exponential, as occurs in the case of fast donor migration (i.e. the hopping model). The rationalization lies in the notion that the donor decay can then be described by a collective macroscopic rate, which can be modeled with rate equations. The self-consistent nature of our data seems to support this justification.

Further self-consistency can be seen in the data in Table 4.5 and Table 4.6. We expect (and observe) that as the sample concentration increases, cross-relaxation between the $W$ and $Y$ levels will tend to increase the cross-relaxation fraction of the $W$ level $\left(\eta_{w}^{c}\right)$ at the expense of the radiative fraction $\left(\eta_{\mathrm{w}}^{\mathrm{rad}}\right)$, leaving the (concentration independent) multiphonon fraction $\left(\eta_{\mathrm{w}}^{M P}\right)$ unchanged. Thus the multiphonon-dominated $b_{\mathrm{wx}}$ remains constant while the cross-relaxation-dominated $b_{w y}$ increases and $b_{w z}$ decreases with increasing concentration. As $\eta_{\mathrm{x}}^{\text {rad }}, \mathrm{b}_{\mathrm{XY}}$, and $\mathrm{b}_{\mathrm{XZ}}$ are unaffected by cross-relaxation, they too remain independent of concentration.

Another self-consistent result is the value of $\eta_{x}^{\text {rad }}$ from Table 4.5. Since level $X$ lies $2350 \mathrm{~cm}^{-1}$ (over 11 phonons) above the $\mathrm{Y}$ level, we expect the multiphonon contribution to the $\mathrm{X}$-level decay to be minimal, making the radiative quantum efficiency near unity. Figure 4.12 shows the values of the radiative quantum efficiencies $\eta_{\mathrm{w}}^{\mathrm{rad}}$, $\eta_{\mathrm{x}}^{\mathrm{rad}}, \eta_{\mathrm{Y}}^{\text {rad }}$ for sample \#1 plotted as a function of $\eta_{\mathrm{w}}^{c}$ according to (3.14)-(3.16). In this plot, the curves reflect the radiative branching ratios $\beta_{\mathrm{JJ}}^{\mathrm{rad}}$ and fluorescence ratios ${ }^{\mathrm{P}} \phi_{\mathrm{J} / \mathrm{K}}$ 
that were actually measured for sample $\# 1$; only $\eta_{\mathrm{w}}^{c}$ (the fraction of the $\mathrm{W}$-level population taking the cross-relaxation pathway) has been allowed to vary independently.

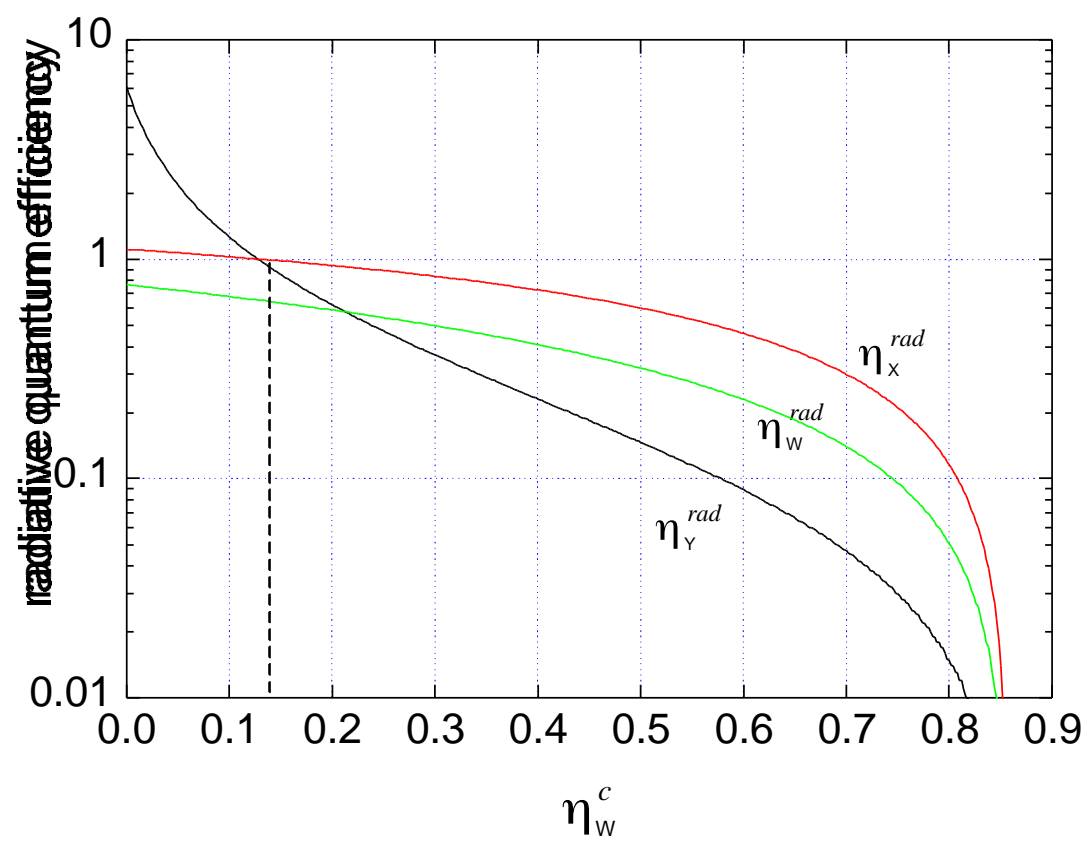

Figure 4.12. The $W, X$, and $Y$ level radiative quantum efficiencies are plotted as a function of the $\mathrm{W}$ level cross-relaxation fraction for sample \#1 according to (3.14)-(3.16). The dashed vertical line indicates the value of $\eta_{\mathrm{w}}^{c}$ determined from lifetime measurements according to (3.5).

We see that $\eta_{\mathrm{x}}^{\text {rad }}$ is near unity at precisely the value of $\eta_{\mathrm{w}}^{c}$ determined from lifetime measurements via (3.5), indicated by the dashed vertical line in the figure. The assumption of a cross-relaxation path with a decay rate of the form $\mathrm{W}^{\mathrm{c}}=k \mathrm{~N}_{0}{ }^{2}$ is critical in obtaining this result, since $\eta_{\mathrm{w}}^{c}=1-W_{\mathrm{w}}^{0} / W_{\mathrm{w}}$ and $W_{\mathrm{w}}^{0}$ is obtained from the straight-line fit to the plot of $W_{\mathrm{w}}$ vs. $\mathrm{N}_{0}^{2}$ (e.g. Figure 4.9). Notice in Figure 4.12 that values of $\eta_{\mathrm{w}}^{c}$ less than 0.13 and greater than 0.85 are unphysical since the radiative quantum efficiencies there are outside the physical boundary $0 \leq \eta^{\mathrm{rad}} \leq 1$. 
The radiative lifetimes, determined from (2.74) as the ratio of the measured lifetime to the radiative quantum efficiency, can also be used as a consistency check since $\tau^{\text {meas }}$ and $\eta^{\text {rad }}$ are independently determined. We expect $\tau^{\text {rad }}$ values to be intrinsic to $\mathrm{KPb}_{2} \mathrm{Cl}_{5}$ and thus independent of concentration. The relatively constant values for the $\mathrm{A}$, $\mathrm{W}, \mathrm{X}$, and $\mathrm{Y}$ levels in Table 4.5 generally point to self-consistency. Samples \#4 and \#5, however, seem to be inconsistent with samples \#1-\#3 for the $Y$ level. The lower values of $\eta_{Y}^{\text {rad }}$ (and the relatively large values of ${ }^{w} \phi_{W / Y}$ and ${ }^{w_{\phi_{W}}}$ in Table 4.4) are attributed to $\mathrm{OH}$ quenching of the $\mathrm{Y}$ level near $3 \mu \mathrm{m}$ in samples \#4 and \#5. A corresponding decrease in the measured $\mathrm{Y}$-level lifetimes for these samples was expected but not observed (although a small decrease was observed). This inconsistency has not yet been resolved. $\mathrm{OH}$ concentration has been estimated based on values for $\mathrm{OH}$ quenching in $\mathrm{Er}^{3+}$-doped phosphate glasses. ${ }^{64}$ The nonradiative decay rate due to $\mathrm{OH}$ impurities, $W^{O H}$, can be written $W^{O H}=k N_{O H} \sigma_{O H}$, where $N^{O H}$ is the $\mathrm{OH}$ concentration, $\sigma_{O H}$ is the $\mathrm{OH}$ absorption cross section, and $k$ is a coefficient with dimensions length/time. From the data in Ref. [64] we can infer $\sigma_{O H}=12.6 \times 10^{-20} \mathrm{~cm}^{2}$ and $k=22 \mathrm{~cm} / \mathrm{s}$. Since $W^{O H}=\eta^{O H} / \tau^{\text {meas }}$, we can use $\eta^{O H}$ and $\tau^{\text {meas }}$ data from Table 4.5 to estimate $N_{O H}=\eta^{O H} /\left(k \sigma_{O H} \tau^{\text {meas }}\right)$. Sample \#1 in Table 4.5 has a $10 \%$ reduction in $\eta^{\text {rad }}$ (presumably) due to $\mathrm{OH}$ quenching, implying $\eta^{O H} \approx 10 \%$. Using $\tau^{\text {meas }} \approx 15 \mathrm{~ms}$ we obtain $N_{O H} \approx 2.4 \times 10^{18} \mathrm{~cm}^{-3}=14 \mathrm{ppm}$. Thus even a small concentration of $\mathrm{OH}$ seems to have a significant effect on the decay kinetics of $\mathrm{KPb}_{2} \mathrm{Cl}_{5}: \mathrm{Dy}^{3+}$.

The small value $(<1 \%$ ) for the A-Level radiative quantum efficiency is surprising since the energy gap to the $\mathrm{W}-$ level of $\sim 1300 \mathrm{~cm}^{-1}$ corresponds to more the 6 phonons. Indeed there may be processes other than nonradiative multiphonon decay at work, as 
evidenced by the fact that Dieke did not observe fluorescence from the $\mathrm{A}$ level in $\mathrm{LaCl}_{3} \cdot{ }^{15}$ The absorption feature near $7.2 \mu \mathrm{m}\left(\sim 1390 \mathrm{~cm}^{-1}\right.$, see Figure 4.5) most likely plays a major role in reducing the radiative decay rate from the $A$ level.

The results of this analysis that can be directly compared with the Judd-Ofelt analysis of Section 4.4 are shown in Table 4.7. The $\mathrm{W}={ }^{6} \mathrm{H}_{9 / 2}+{ }^{6} \mathrm{~F}_{11 / 2}$ (and $\mathrm{A}={ }^{6} \mathrm{H}_{7 / 2}+{ }^{6} \mathrm{~F}_{9 / 2}$ ) level data was calculated assuming the constituent levels were in thermal equilibrium.

Table 4.7. Measured data from Table 4.3 and Table 4.5 compared to the Judd-Ofelt data from Table 4.2. Average values were used for the measured data whenever applicable.

\begin{tabular}{|c|c|c|c|c|c|c|c|}
\hline \multirow[b]{2}{*}{ Transition } & \multirow[b]{2}{*}{$\lambda_{\text {peak }}(\mu \mathrm{m})$} & \multicolumn{2}{|c|}{ Judd-Ofelt calc'n } & \multirow{2}{*}{$\frac{\sigma_{\text {em,peak }}{ }^{*}}{\left(10^{-20} \mathrm{~cm}^{2}\right)}$} & \multicolumn{2}{|c|}{ Measured data } & \multirow{2}{*}{$\frac{\sigma_{\text {em,peak }}{ }^{*}}{\left(10^{-20} \mathbf{c m}^{2}\right)}$} \\
\hline & & $\beta^{\mathrm{rad}}$ & $\tau^{\mathrm{rad}}(\mathrm{ms})$ & & $\beta^{\mathrm{rad}}$ & $\tau^{\mathrm{rad}}(\mathrm{ms})$ & \\
\hline $\mathrm{W} \rightarrow \mathrm{X}$ & 5.5 & 0.010 & \multirow{3}{*}{1.09} & 0.87 & 0.013 & \multirow{3}{*}{1.18} & 1.04 \\
\hline $\mathrm{W} \rightarrow \mathrm{Y}$ & 2.4 & 0.108 & & 0.74 & 0.080 & & 0.50 \\
\hline $\mathrm{W} \rightarrow \mathrm{Z}$ & 1.3 & 0.882 & & 1.00 & 0.907 & & 0.95 \\
\hline$X \rightarrow Y$ & 4.3 & 0.091 & \multirow{2}{*}{4.24} & 0.76 & 0.128 & \multirow{2}{*}{6.54} & 0.69 \\
\hline$X \rightarrow Z$ & 1.7 & 0.909 & & 0.65 & 0.872 & & 0.40 \\
\hline $\mathrm{Y} \rightarrow \mathrm{Z}$ & 2.8 & 1.000 & 11.8 & 0.81 & 1.000 & 15.7 & 0.61 \\
\hline
\end{tabular}

The Judd-Ofelt predictions of radiative branching ratios were generally good (within $10 \%$ ) but the radiative lifetimes have been somewhat underestimated. The Judd-Ofelt method is considered generally accurate to within about $20 \%$. The error could be much higher in this case owing to the sensitivity of the results to the hypersensitive $1.3 \mu \mathrm{m}$ transition.

The branching ratios and radiative lifetimes measured here can be used to obtain more accurate emission cross section data via (2.20). In fact, these cross sections require only the measured refractive index, fluorescence lifetimes, and calibrated emission spectra of the selectively pumped energy levels. The measured peak cross sections are 
included in Table 4.7, and in general are smaller than those predicted by the Judd-Ofelt data.

\subsection{Other Chloride Samples}

Although $\mathrm{KPb}_{2} \mathrm{Cl}_{5}: \mathrm{Dy}^{3+}$ was the focus of our research, other chloride samples were investigated. These included $\mathrm{RbPb}_{2} \mathrm{Cl}_{5}: \mathrm{Dy}^{3+}, \mathrm{KPb}_{2} \mathrm{Cl}_{5}: \mathrm{Tb}^{3+}, \mathrm{KPb}_{2} \mathrm{Cl}_{5}: \mathrm{Er}^{3+}$, and $\mathrm{KPb}_{2} \mathrm{Cl}_{5}: \mathrm{Nd}^{3+}$.

\subsection{1 $\mathrm{RbPb}_{2} \mathrm{Cl}_{5}: \mathrm{Dy}^{3+}$}

$\mathrm{RbPb}_{2} \mathrm{Cl}_{5}$ belongs to the structural homogeneous family $\mathrm{APb}_{2} \mathrm{X}_{5}$, where $\mathrm{A}=\mathrm{K}, \mathrm{Rb}, \mathrm{NH}_{4}$ and $\mathrm{X}=\mathrm{Cl}, \mathrm{Br}$. This emission properties of $\mathrm{Pb}^{2+}$ centers in this material were studied previously. ${ }^{65}$ As $\mathrm{Rb}$ is heavier than $\mathrm{K}$, it was expected that the maximum phonon energy in $\mathrm{RbPb}_{2} \mathrm{Cl}_{5}$ would be even lower than that found for $\mathrm{KPb}_{2} \mathrm{Cl}_{5}$. The growth method was the same as that for $\mathrm{KPb}_{2} \mathrm{Cl}_{5}$ described in Section 4.1. The average $\mathrm{Pb}-\mathrm{K}$ and $\mathrm{Pb}-\mathrm{Rb}$ distance (> 4.6 (1) ) is sufficiently large to make the substitution of $\mathrm{Rb}$ for $\mathrm{K}$ ions possible without affecting the structure. ${ }^{14}$ In general, the crystal quality seemed to match that of $\mathrm{KPb}_{2} \mathrm{Cl}_{5}$, as well as it's insensitivity to moisture. While the maximum phonon energy was not measured directly (via Raman scattering), it can be estimated from the IR cut-off wavelength (see Appendix E). The lifetime of the $\mathrm{W}$ level in $\mathrm{RbPb}_{2} \mathrm{Cl}_{5}: \mathrm{Dy}^{3+}$ was measured to be $0.83 \mathrm{~ms}$, considerably longer than that measured in $\mathrm{KPb}_{2} \mathrm{Cl}_{5}: \mathrm{Dy}^{3+}(0.72$ $\mathrm{ms}$ is the lowest concentration sample), most likely indicating lower multi-phonon decay. The cut-off wavelength ( $10 \%$ transmission) for $\mathrm{RbPb}_{2} \mathrm{Cl}_{5}$ was measured to be $21 \mu \mathrm{m}$ (470 $\mathrm{cm}^{-1}$ ), similar to $\mathrm{KPb}_{2} \mathrm{Cl}_{5}$. Thus it is unclear (without the Raman spectrum) whether the maximum phonon energy is lower than that of $\mathrm{KPb}_{2} \mathrm{Cl}_{5}$. The problem with $\mathrm{RbPb}_{2} \mathrm{Cl}_{5}$ is 
that it did not seem to incorporate $\mathrm{Dy}^{3+}$ as well as $\mathrm{KPb}_{2} \mathrm{Cl}_{5}$, although with concentrated growth efforts this could be a good long-wavelength host material. The absorption and emission spectra for $\mathrm{RbPb}_{2} \mathrm{Cl}_{5}: \mathrm{Dy}^{3+}$ are shown in Figure 4.13 and Figure 4.14, respectively. The absorption spectra was taken along three mutually perpendicular axes of the biaxial indicatrix, identified at extinction positions between crossed polarizers. The emission cross section was calculated with (2.20) using unpolarized emission data.

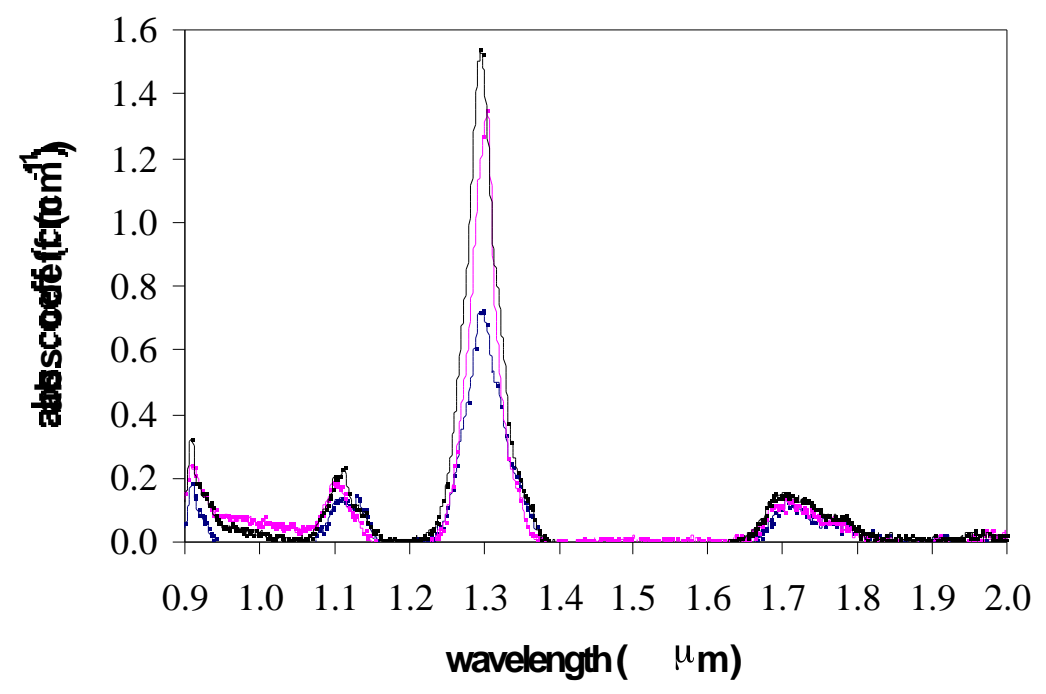

Figure 4.13. Absorption spectrum for $\mathrm{RbPb}_{2} \mathrm{Cl}_{5}: \mathrm{Dy}^{3+}$ polarized along three mutually perpendicular axes of the biaxial indicatrix.

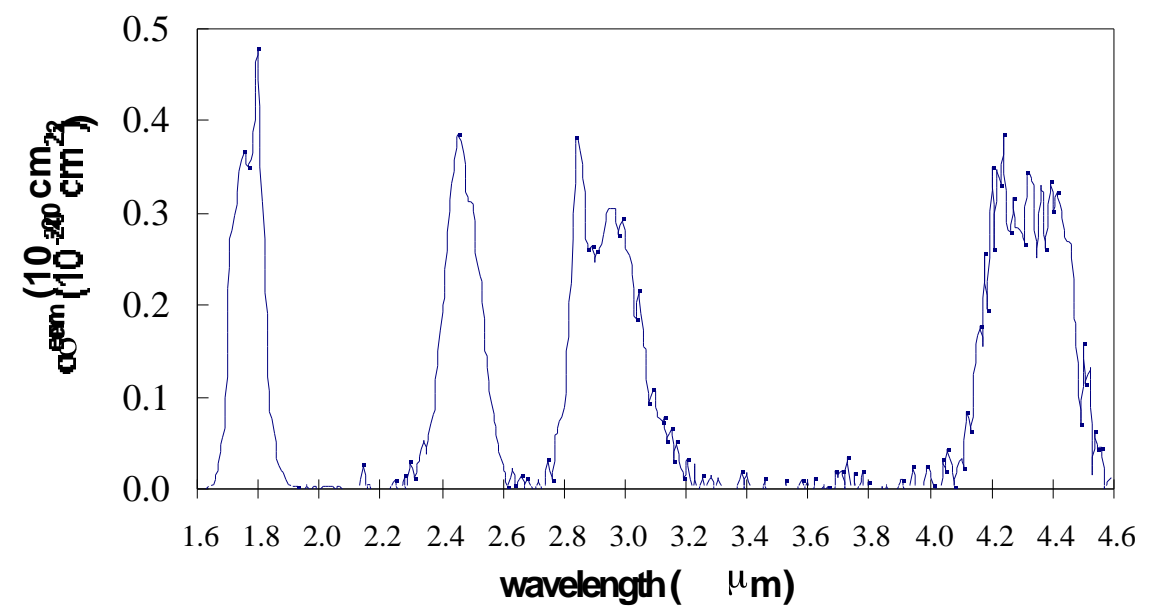

Figure 4.14. Unpolarized emission cross section of $\mathrm{RbPb}_{2} \mathrm{Cl}_{5}: \mathrm{Dy}^{3+}$. The sample was pumped at $1.3 \mu \mathrm{m}$. 


\subsection{2 $\mathrm{KPb}_{2} \mathrm{Cl}_{5}: \mathrm{Tb}^{3+}$}

The $\mathrm{Tb}^{3+}$ ion was of interest to us for its long-wavelength transitions, most notably the $10-\mu \mathrm{m}{ }^{7} \mathrm{~F}_{3} \rightarrow{ }^{7} \mathrm{~F}_{4}$ transition, the $7.5-\mu \mathrm{m}{ }^{7} \mathrm{~F}_{4} \rightarrow{ }^{7} \mathrm{~F}_{5}$ transition, and the $5.5-\mu \mathrm{m}{ }^{7} \mathrm{~F}_{5} \rightarrow{ }^{7} \mathrm{~F}_{6}$ transition. An energy-level diagram is provided in Figure 4.15. The measured lifetime of the ${ }^{7} \mathrm{~F}_{5}$ level is $3.31 \mathrm{~ms}$.

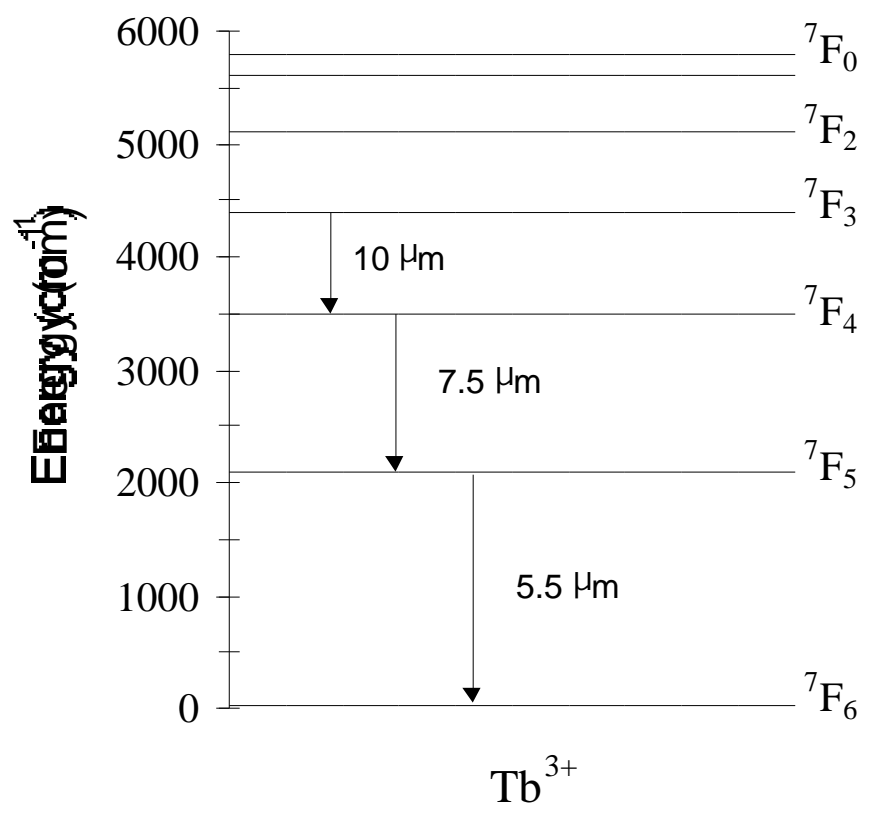

Figure 4.15. Energy-level diagram of $\mathrm{Tb}^{3+}$. The measured lifetimes are indicated on the diagram.

The absorption and emission spectra for $\mathrm{KPb}_{2} \mathrm{Cl}_{5}: \mathrm{Tb}^{3+}$ are shown in Figure 4.16 and Figure 4.17, respectively. Emission from the ${ }^{7} \mathrm{~F}_{4}$ level was expected but not observed. The reason for this is most likely $\mathrm{NH}_{4}{ }^{+}$impurities introduced in the purification process. See Figure 4.16 and the $\mathrm{KPb}_{2} \mathrm{Cl}_{5}: \mathrm{Er}^{3+}$ section below for details. $\mathrm{NH}_{4}{ }^{+}$concentration can be estimated from integrated absorption data in the literature. For estimation purposes, we can use the integrated absorption coefficient of $s=5.6 \times 10^{-18} \mathrm{~cm} / \mathrm{molecule}$ for $\mathrm{NH}_{3}$ 
near $6.1 \mu \mathrm{m}$ given in Ref. [66]. The concentration of $\mathrm{NH}_{4}{ }^{+}\left(N_{0}\right)$ is then given by $N_{0}=$ $\alpha \cdot \Delta v / s$, where $\Delta v$ is the bandwidth $\left(\mathrm{cm}^{-1}\right)$ and $\alpha$ is the coefficient $\left(\mathrm{cm}^{-1}\right)$ of the impurity absorption. From Figure 4.16 we infer $\alpha \approx 0.02 \mathrm{~cm}^{-1}$ and $\Delta v \approx 40 \mathrm{~cm}^{-1}$, so that $N_{0} \approx 1.4 \times$ $10^{17} \mathrm{~cm}^{-3} \approx 1 \mathrm{ppm}$.

Attempts were made at laser action on the ${ }^{7} \mathrm{~F}_{5} \rightarrow{ }^{7} \mathrm{~F}_{6}$ 3-level transition at $5.5 \mu \mathrm{m}$, but were unsuccessful. The damage threshold of $\sim 13 \mathrm{~J} / \mathrm{cm}^{2}$ was reached before laser oscillation occurred. The laser threshold was estimated at $\sim 8 \mathrm{~J} / \mathrm{cm}^{2}$ based on the following data: pump wavelength $=2 \mu \mathrm{m}$, pump spot size $=300 \mu \mathrm{m}$, laser spot size $=418$ $\mu \mathrm{m}$, emission cross section $\sim 0.2 \times 10^{-20} \mathrm{~cm}^{2}$, passive losses $=2 \%$ per round-trip, absorbed pump power $\sim 30 \%$, and an upper-state lifetime of $3.3 \mathrm{~ms}$.

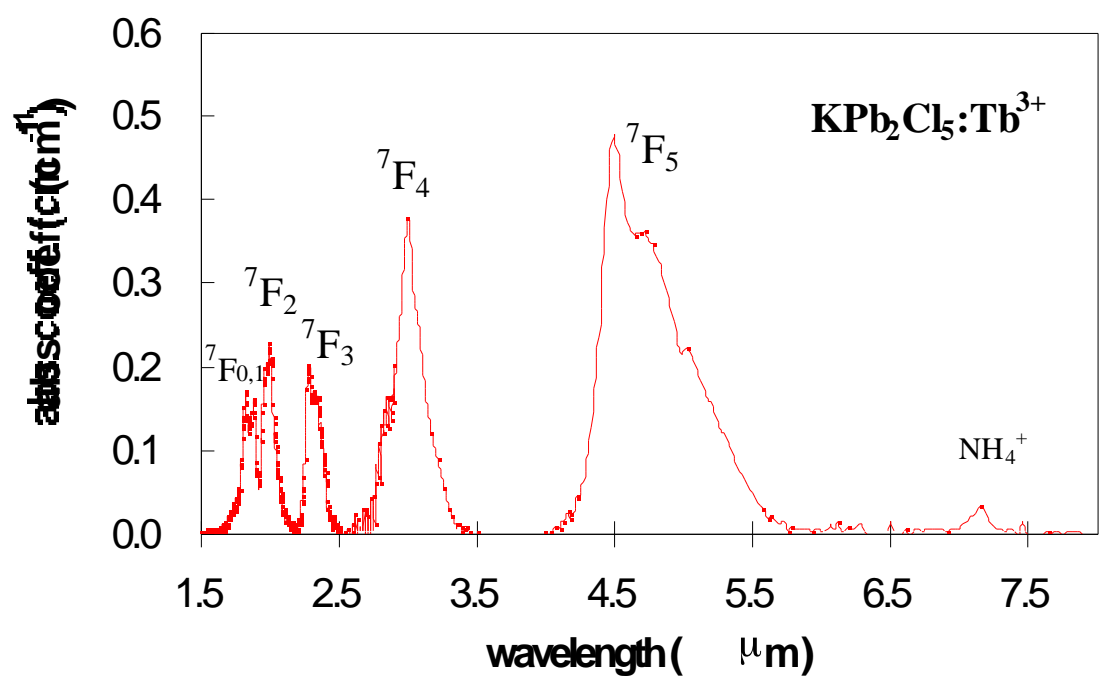

Figure 4.16. $\mathrm{KPb}_{2} \mathrm{Cl}_{5}: \mathrm{Tb}^{3+}$ absorption spectrum. The ground-state level is ${ }^{7} \mathbf{F}_{6}$. The sample length was $5.9 \mathrm{~mm}$ and the nominal doping was $2.5 \mathrm{~mol} \%$. The $\mathrm{NH}_{4}{ }^{+}$impurity absorption is visible near $7.2 \mu \mathrm{m}$. 


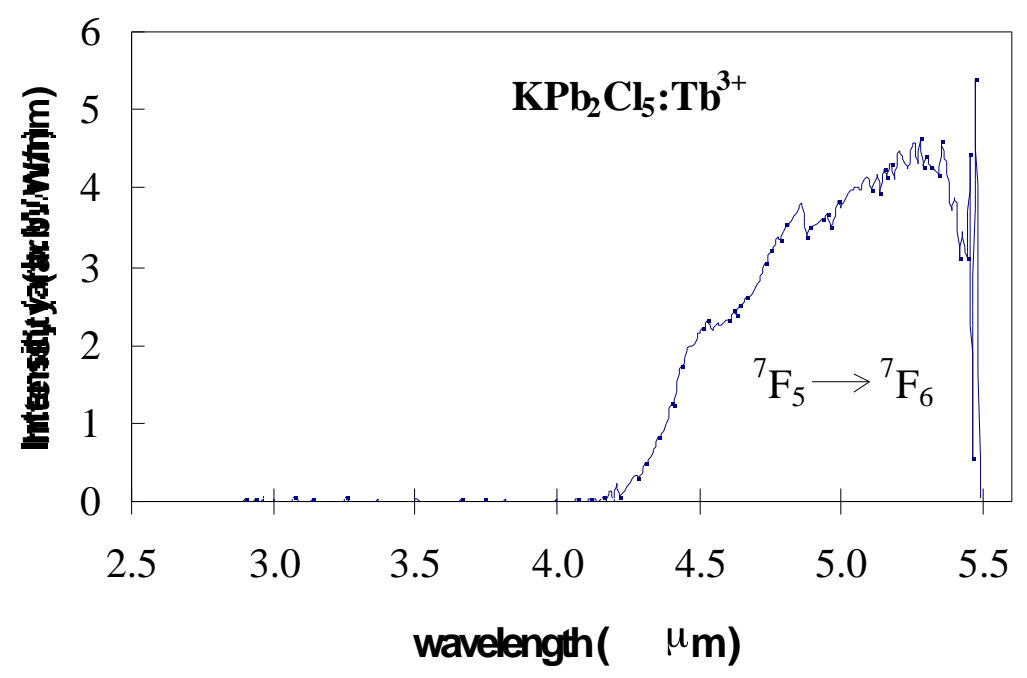

Figure 4.17. Calibrated $\mathrm{KPb}_{2} \mathrm{Cl}_{5}: \mathrm{Tb}^{3+}$ emission spectrum. The cutoff near $5.5 \mu \mathrm{m}$ is due to the cutoff of the InSb detector. The pump level is the ${ }^{7} \mathbf{F}_{2}$ at $2.0 \mu \mathrm{m}$.

\subsection{3 $\mathrm{KPb}_{2} \mathrm{Cl}_{5}: \mathrm{Er}^{3+}$}

$\mathrm{KPb}_{2} \mathrm{Cl}_{5}: \mathrm{Er}^{3+}$ was of interest due to the $\left({ }^{4} \mathrm{I}_{9 / 2} \rightarrow{ }^{4} \mathrm{I}_{11 / 2}\right) 4.5 \mu \mathrm{m}$ transition shown in Figure 4.18. The absorption spectrum is shown in Figure 4.19.

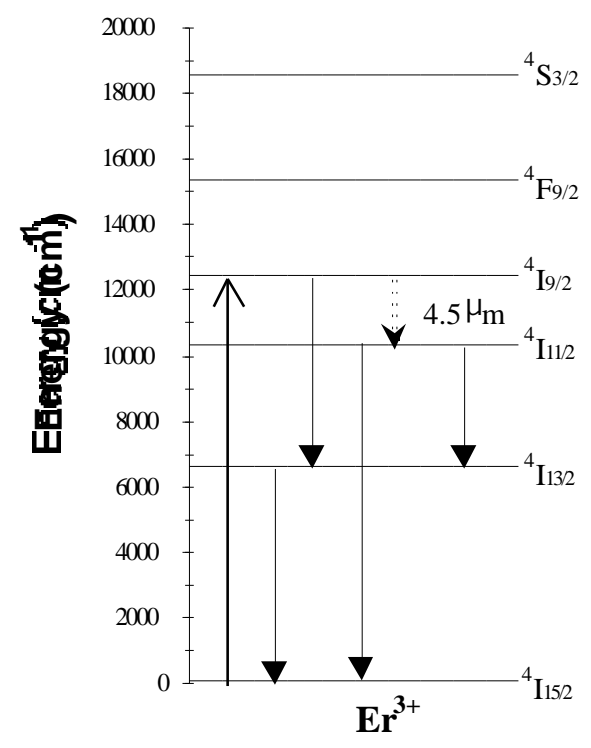

Figure 4.18. Energy-level diagram of $\mathrm{Er}^{3+}$. The thick upward arrow indicates the pumping transition, while the thin downward arrows indicate observed luminescence. 
The features at 2, 3, and $7 \mu \mathrm{m}$, corresponding to $\mathrm{NH}_{4}^{+}$impurities introduced in purification of the crystals where $\mathrm{NH}_{4} \mathrm{Cl}$ is used as a chlorine agent. The emission spectrum is shown in Figure 4.20, where the ${ }^{4} \mathrm{I}_{9 / 2}$ level was pumped at $0.81 \mu \mathrm{m}$. Emission on the ${ }^{4} \mathrm{I}_{9 / 2} \rightarrow{ }^{4} \mathrm{I}_{11 / 2}$ transition at $4.5 \mu \mathrm{m}$ was not observed, probably due to a small branching ratio.
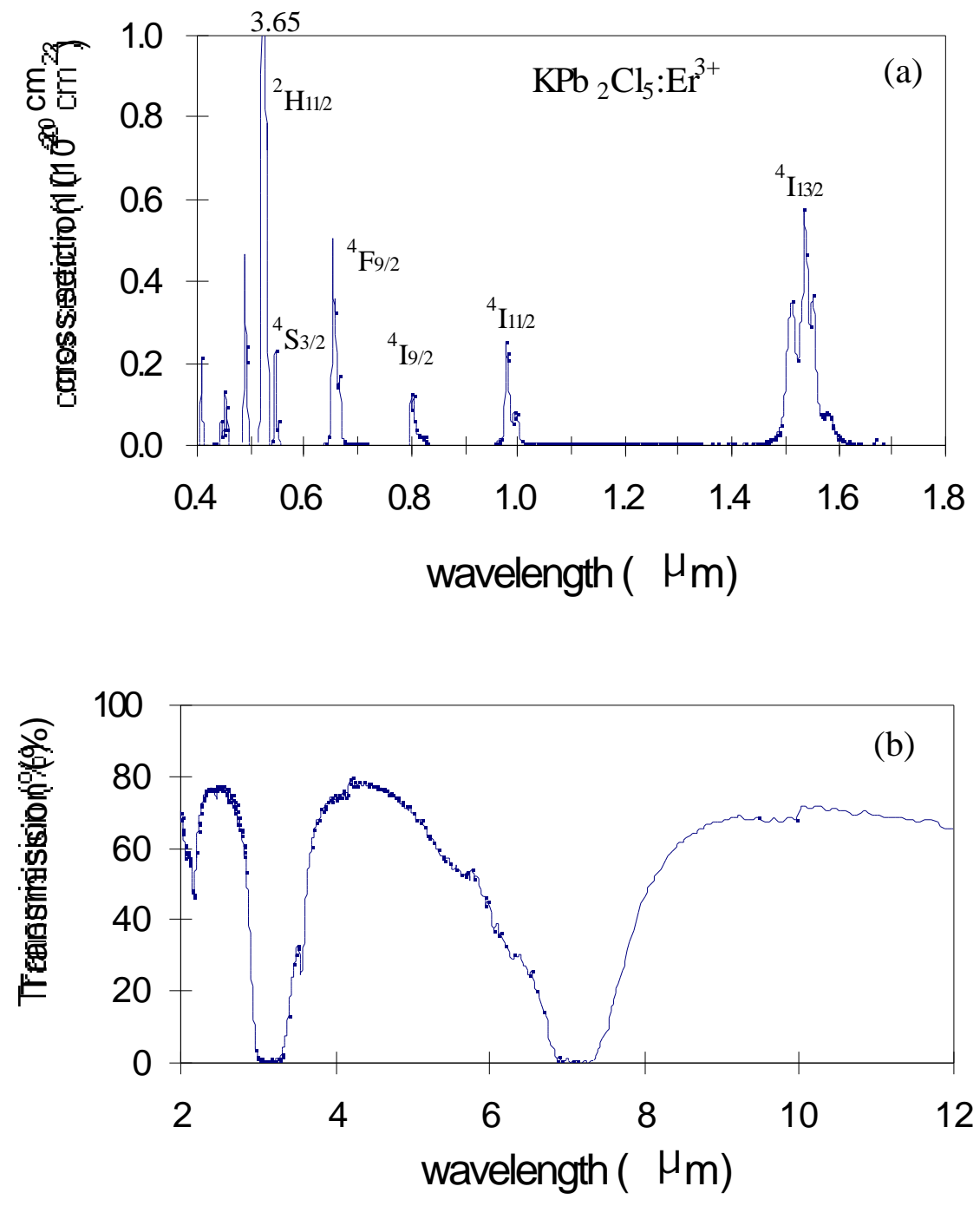

Figure 4.19. $\mathrm{KPb}_{2} \mathrm{Cl}_{5}: \mathrm{Er}^{3+}$ absorption spectrum. (a) $0.4-1.8 \mu \mathrm{m}$ spectra displays identifiable $\mathrm{Er}^{3+}$ features. (b) 2 - 12- $\mu \mathrm{m}$ spectra (transmission) shows the $\mathrm{NH}_{4}{ }^{+}$absorption features at 3 and $7 \mu \mathrm{m}$, and the combination near $2 \mu \mathrm{m}$. 

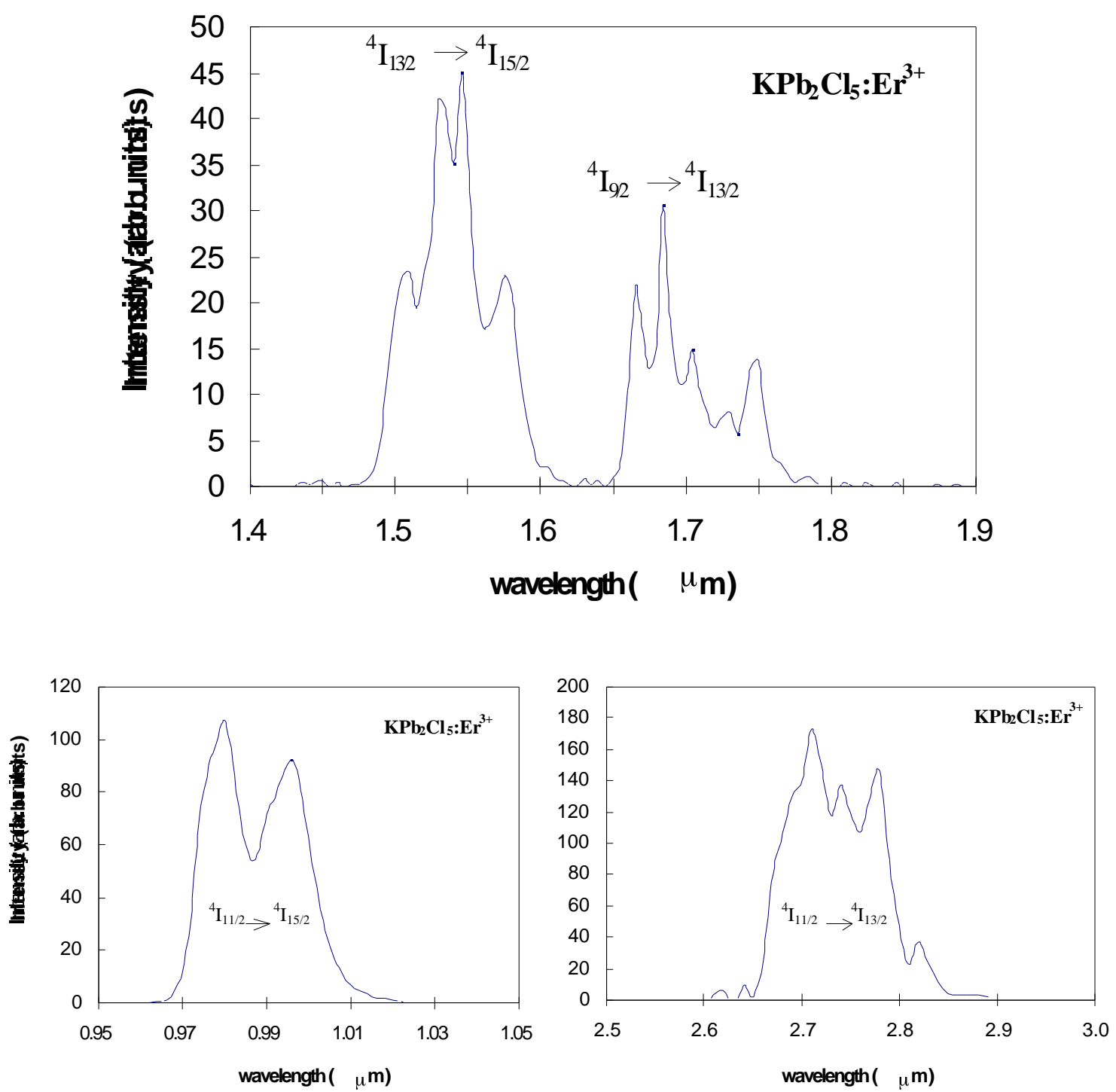

Figure 4.20. $\mathrm{KPb}_{2} \mathrm{Cl}_{5}: \mathrm{Er}^{3+}$ emission spectrum. The sample was pumped at $0.81 \mu \mathrm{m}$. Emission on the ${ }^{4} \mathbf{I}_{9 / 2} \rightarrow{ }^{4} \mathbf{I}_{11 / 2}$ transition at $4.5 \mu \mathrm{m}$ was not observed, probably due to a small branching ratio.

\subsection{4 $\mathrm{KPb}_{2} \mathrm{Cl}_{5}: \mathrm{Nd}^{3+}$}

$\mathrm{KPb}_{2} \mathrm{Cl}_{5}: \mathrm{Nd}^{3+}$, whose absorption spectrum is shown in Figure 4.21, was included in this study since the ${ }^{4} \mathrm{~F}_{5 / 2}$ and ${ }^{4} \mathrm{~F}_{3 / 2}$ levels of $\mathrm{Nd}^{3+}$ (see inset of Figure 4.21) provide a 
convenient energy gap $\left(1030 \mathrm{~cm}^{-1}\right.$ peak-to-peak) for the multiphonon-relaxation plot of the next section.

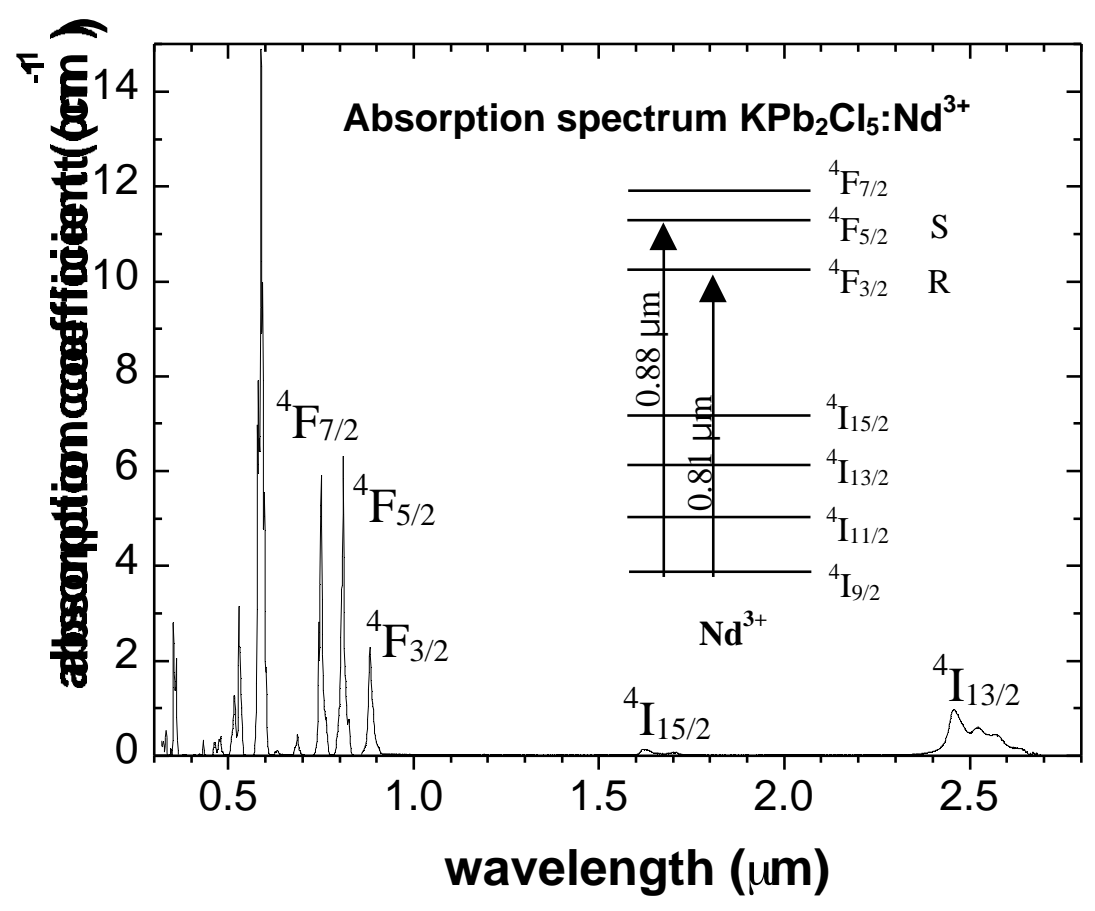

Figure 4.21. $\mathrm{Nd}^{3+}$ absorption spectrum and energy levels. Note the letter designations of the ${ }^{4} \mathbf{F}_{5 / 2}$ and ${ }^{4} \mathbf{F}_{3 / 2}$ levels.

The analysis to determine the radiative quantum efficiencies (similar to Section 4.7) for these levels is quite simple, since we may assume the radiative quantum efficiency for the ${ }^{4} \mathrm{~F}_{3 / 2}$ level is $\approx 1$, and the radiative branching ratio between the levels is $\approx 0$, so that multiphonon decay is the only pathway connecting the two levels. Labeling the $\mathrm{Nd}^{3+}$ ${ }^{4} F_{5 / 2}$ level " $S$ " and the ${ }^{4} F_{3 / 2}$ level " $R$ ", the analogous result to (3.15) is

$$
\eta_{\mathrm{S}}^{\mathrm{rad}}=\frac{1}{\mathrm{~s}_{\phi_{\mathrm{R} / \mathrm{S}}}+1} .
$$

The 'S-pumped' emission data for $\mathrm{Nd}^{3+}$ is shown in Figure 4.22, where emission peaks originating from the $\mathrm{S}$ level at $0.81 \mu \mathrm{m}, 0.95 \mu \mathrm{m}$, and $1.18 \mu \mathrm{m}$ are visible. A CW Ti:Sapphire laser tuned to $0.81 \mu \mathrm{m}$ was used to pump the sample, and a liquid-nitrogen 
cooled InSb detector (along with appropriate bandpass filters) was used to record the emission from $0.83-1.8 \mu \mathrm{m}$ at the exit port of a 1-meter, $1-\mu \mathrm{m}$ blazed grating monochromator.

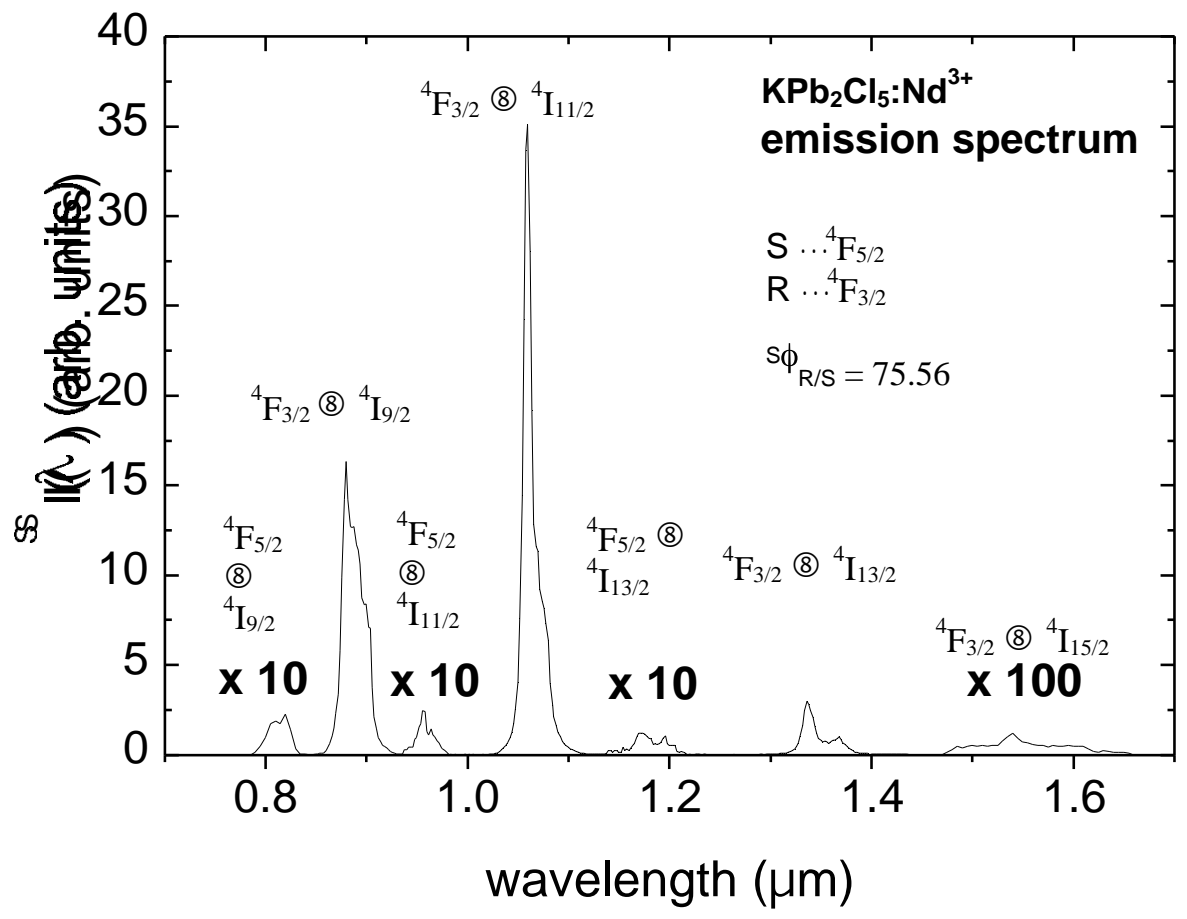

Figure 4.22. Emission spectrum obtained by direct excitation of the $S$ level of $\mathrm{KPb}_{2} \mathrm{Cl}_{5}: \mathrm{Nd}^{3+}$. Emission from both the ${ }^{4} \mathrm{~F}_{3 / 2}$ " $\mathrm{R}$ " and ${ }^{4} \mathrm{~F}_{5 / 2}$ " $S$ " level is observed. The data infer a fluorescence ratio ${ }_{\phi_{R} / \mathrm{s}}$ of 75.56.

The emission near $810 \mathrm{~nm}$ was obtained on a second run spanning $0.78-1.1 \mu \mathrm{m}$, using the Ti:Sapphire laser tuned to $0.77 \mu \mathrm{m}$ (pumping the ${ }^{4} \mathrm{~F}_{7 / 2}$ level) and a dry-ice cooled PMT. The two runs were then combined by matching the emission peaks in the overlapping region. The value of ${ }^{s} \phi_{R / S}$ was found to be 75.56 , giving $\eta_{S}^{\text {rad }}=0.013$. The fluorescence lifetime for the $S$ level was measured to be $3.4 \mu \mathrm{s}$, while that of the R level was measured to be $340 \mu \mathrm{s}$. Since multiphonon decay is the major contributor to lifetime 
reduction of the $S$ level, this lifetime data suggests $\eta_{S}^{\text {rad }}=0.01$, in good agreement with the above result.

Emission spectra were also obtained from the ${ }^{4} \mathrm{I}_{15 / 2},{ }^{4} \mathrm{I}_{13 / 2},{ }^{4} \mathrm{I}_{11 / 2}$ levels of $\mathrm{KPb}_{2} \mathrm{Cl}_{5}: \mathrm{Nd}^{3+}$ near $5 \mu \mathrm{m}$. These data were obtained with $0.8-\mu \mathrm{m}$ pumping, a $4-\mu \mathrm{m}$ blazed grating, and a 77K InSb detector. This data is shown in Figure 4.23.

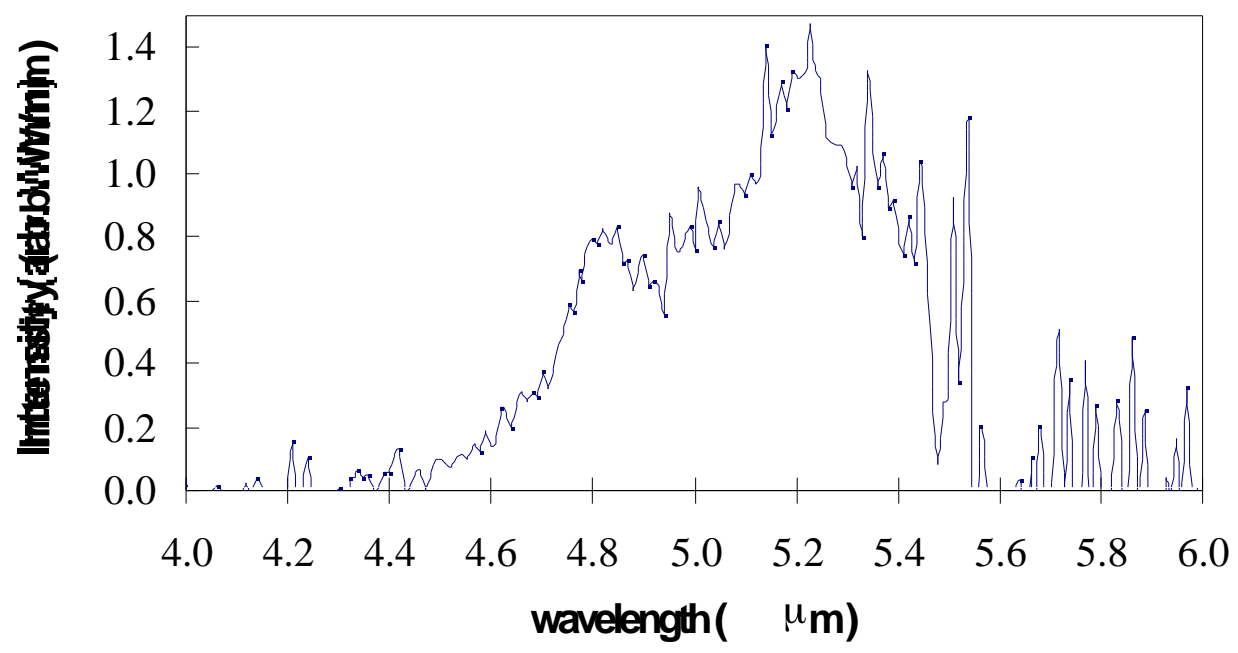

Figure 4.23. Calibrated emission spectrum of $\mathrm{KPb}_{2} \mathrm{Cl}_{5}: \mathrm{Nd}^{3+}$ near $5 \mu \mathrm{m}$. The sample was pumped at $0.8 \mu \mathrm{m}$. This data represents emission from the ${ }^{4} \mathbf{I}_{15 / 2},{ }^{4} \mathbf{I}_{13 / 2}$, ${ }^{4} \mathbf{I}_{11 / 2}$ levels. The data is truncated due to the InSb detector cutoff near $5.5 \mu \mathrm{m}$.

Since the overlap of the emission from the ${ }^{4} \mathrm{I}_{15 / 2},{ }^{4} \mathrm{I}_{13 / 2},{ }^{4} \mathrm{I}_{11 / 2}$ levels is great, cross relaxation is expected to play a large role in the decay dynamics. Whereas estimates using reduced matrix elements estimate radiative lifetimes in excess of $30 \mathrm{~ms}$ for these levels, a measured lifetime of only $2.5 \mathrm{~ms}$ was obtained for the $5 \mu \mathrm{m}$ output. This indeed points to the existence of cross relaxation, and thus to $\mathrm{Nd}^{3+}: \mathrm{KPb}_{2} \mathrm{Cl}_{5}$ as a good candidate to study cross relaxation in solid-state materials. 


\subsection{Multiphonon decay vs. Energy Gap}

The analysis of Section 4.7 permits a calculation of the nonradiative (multiphonon) decay rates so that we may plot multiphonon emission rate vs. energy gap and deduce the phenomenological parameters described by the 'energy-gap law', as stated in (2.30). Using the $\mathrm{Dy}^{3+}$ quantum efficiency and measured lifetime data from Table 4.5 and the $\mathrm{Nd}^{3+}$ data from Section 4.8.4, the multiphonon decay rates $\mathrm{W}^{\mathrm{MP}}$ can be determined as $\mathrm{W}^{\mathrm{MP}}=\eta^{\mathrm{MP}} / \tau^{\text {meas }}$. Combining this with the energy gaps calculated (peak-to-peak) from the energy levels shown in Figure 4.6, we obtain the plot shown in Figure 4.24. The fitted parameters for $\mathrm{KPb}_{2} \mathrm{Cl}_{5}$ are $B=3.721 \times 10^{9} \mathrm{~s}^{-1}$ and $\beta=1.156 \times 10^{-2} \mathrm{~cm}$. The data point at $\Delta \mathrm{E}=1315 \mathrm{~cm}^{-1}$ arising from the $\mathrm{A}$-level data was not included in the fit.

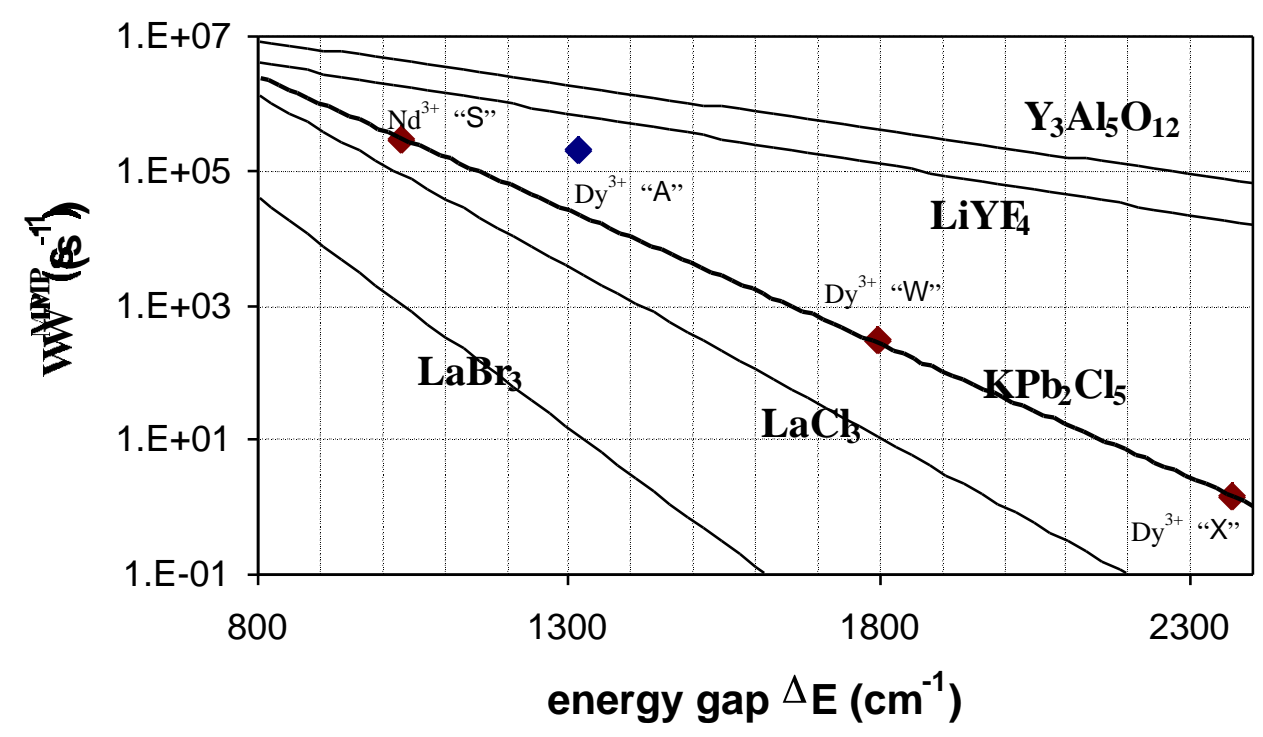

Figure 4.24. Multiphonon relaxation rate vs. energy gap in $\mathrm{KPb}_{2} \mathrm{Cl}_{5}$ (diamonds). The source of each data point in indicated on the figure. The curves for YAG, YLF, $\mathrm{LaCl}_{3}$, and $\mathrm{LaBr}_{3}$ are shown for comparison. These data are taken from Ref. [40].

As discussed in Section 4.7, it is believed the relatively weak emission from this level involves processes other than just nonradiative multiphonon decay, most likely 
quenching due to the $\mathrm{H}_{2} \mathrm{O}$ vibration near $1400 \mathrm{~cm}^{-1}$. It is notable that Dieke also observed fluorescence from the ${ }^{4} \mathrm{~F}_{5 / 2}$ " $\mathrm{S}$ " level in $\mathrm{LaCl}_{3}: \mathrm{Nd}^{3+}\left(\Delta \mathrm{E} \approx 1000 \mathrm{~cm}^{-1}\right)$. For comparison, plots of $\mathrm{W}^{\mathrm{MP}}$ vs. Energy Gap for other hosts ${ }^{40}$ are included in Figure 4.24. $\mathrm{KPb}_{2} \mathrm{Cl}_{5}$ is comparable to $\mathrm{LaCl}_{3}$ in its multiphonon decay characteristics.

From the multiphonon plot we can conclude that to achieve at least $1 \%$ radiative quantum efficiency for a typical radiative rate of $10^{3} \mathrm{~s}^{-1}$ requires a multiphonon rate of at most $10^{5} \mathrm{~s}^{-1}$, which occurs near $\Delta \mathrm{E}=1150 \mathrm{~cm}^{-1}$ in $\mathrm{KPb}_{2} \mathrm{Cl}_{5}$, which corresponds to a wavelength of $\sim 9 \mu \mathrm{m}$. This is consistent with the measured radiative quantum efficiency of $1.3 \%$ for the $1030 \mathrm{~cm}^{-1}$ gap in $\mathrm{KPb}_{2} \mathrm{Cl}_{5}: \mathrm{Nd}^{3+}$.

To achieve $>8-\mu \mathrm{m}$ lasing we thus need to find a suitable $>8-\mu \mathrm{m}$ rare-earth transition. A suitable transition would need a radiative quantum efficiency greater than $1 \%$ and an emission cross section greater than $\sim 1 \times 10^{-20} \mathrm{~cm}^{2}$. Possible candidates are the $\mathrm{Tb}^{3+}{ }^{7} \mathrm{~F}_{3} \rightarrow{ }^{7} \mathrm{~F}_{4}$ transition, the $\mathrm{Eu}^{3+}{ }^{7} \mathrm{~F}_{6} \rightarrow{ }^{7} \mathrm{~F}_{5}$ transition, and the $\mathrm{Dy}^{3+}{ }^{6} \mathrm{H}_{5 / 2} \rightarrow{ }^{6} \mathrm{H}_{7 / 2}$ transition. These transitions, however, are longer than $9 \mu \mathrm{m}$ and may require a host with an even lower effective phonon energy than $\mathrm{KPb}_{2} \mathrm{Cl}_{5}$.

\subsection{Conclusions}

$\mathrm{KPb}_{2} \mathrm{Cl}_{5}$ has been grown in large single crystals of good optical quality, and characterized in terms of optical properties. A maximum phonon energy of $200 \mathrm{~cm}^{-1}$ has been determined, and an indices of refraction near 2.0 have been measured. A Judd-Ofelt analysis has been performed to determine radiative lifetimes and branching ratios, which were compared with experimentally determined values. The analysis indicates laser action to $9 \mu \mathrm{m}$ could theoretically be supported in this host. Other rare-earth dopants were also studied, including $\mathrm{Tb}^{3+}, \mathrm{Er}^{3+}$, and $\mathrm{Nd}^{3+}$. This host readily incorporated all three 
ions (to about 2 mol.\%), suggesting good incorporation of all rare-earth ions. $\mathrm{RbPb}_{2} \mathrm{Cl}_{5}: \mathrm{Dy}^{3+}$ was also studied, and although it did not seem to incorporate $\mathrm{Dy}^{3+}$ as well as $\mathrm{KPb}_{2} \mathrm{Cl}_{5}$, with increased efforts $\mathrm{RbPb}_{2} \mathrm{Cl}_{5}$ may also prove to be a good mid-IR host. 


\section{$5 \quad \mathrm{KPb}_{2} \mathrm{Cl}_{5}$ Laser Results}

\section{$5.1 \mathrm{Dy}^{3+}$ at $2.4 \mu \mathrm{m}$}

$\mathrm{KPb}_{2} \mathrm{Cl}_{5}: \mathrm{Dy}^{3+}$ was of interest to us as a $4.3 \mu \mathrm{m}(\mathrm{W} \rightarrow \mathrm{X})$ laser, see Figure 5.1. To populate the upper level, both $\mathrm{W}$-level pumping (at $1.3 \mu \mathrm{m}$ ) and $\mathrm{X}$-level pumping (at 1.7 $\mu \mathrm{m})$ were considered. However, W-level pumping was ruled out due to insufficient nonradiative transfer from the $\mathrm{W}$ to the $\mathrm{X}$ level, and $\mathrm{X}$-level pumping was ruled out due to insufficient pump energy at $1.7 \mu \mathrm{m}$. A Co: $\mathrm{MgF}_{2}$ laser was cooled to $-30^{\circ} \mathrm{C}$ (DowCorning ${ }^{\mathrm{TM}}$ Syltherm ${ }^{\circledR}$ replaced the water coolant) in order to increase the laser energy output near $1.7 \mu \mathrm{m}$. The absorption cross section near $1.7 \mu \mathrm{m}$ for $\mathrm{KPb}_{2} \mathrm{Cl}_{5}: \mathrm{Dy}^{3+}$ is shown in Figure 5.2. Our best effort produced only a few millijoules of output energy at 1.74 $\mu \mathrm{m}$, while a $4.3-\mu \mathrm{m}$ laser threshold was estimated at near $20 \mathrm{~mJ}$ of incident energy at that wavelength.

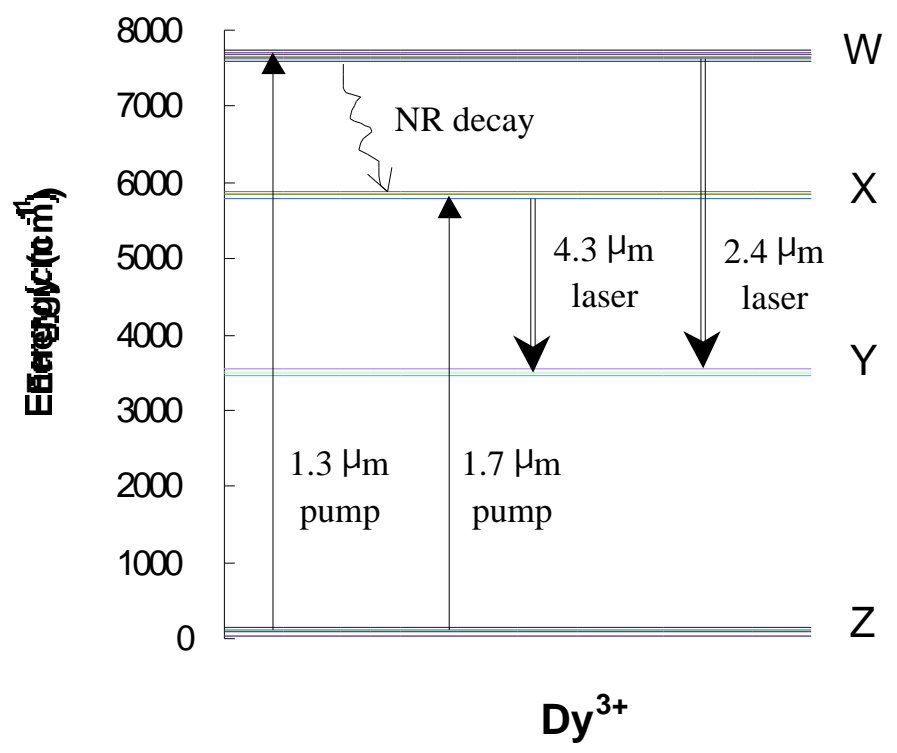

Figure 5.1. $\mathrm{Dy}^{3+}$ energy levels and laser schemes. The upper level of the 4.3- $\mu \mathrm{m}$ laser can be pumped directly at $1.7 \mu \mathrm{m}$ or indirectly at $1.3 \mu \mathrm{m}$ with a subsequent NR decay. The upper level of the 2.4- $\mu \mathrm{m}$ laser is pumped directly at $1.3 \mu \mathrm{m}$. 


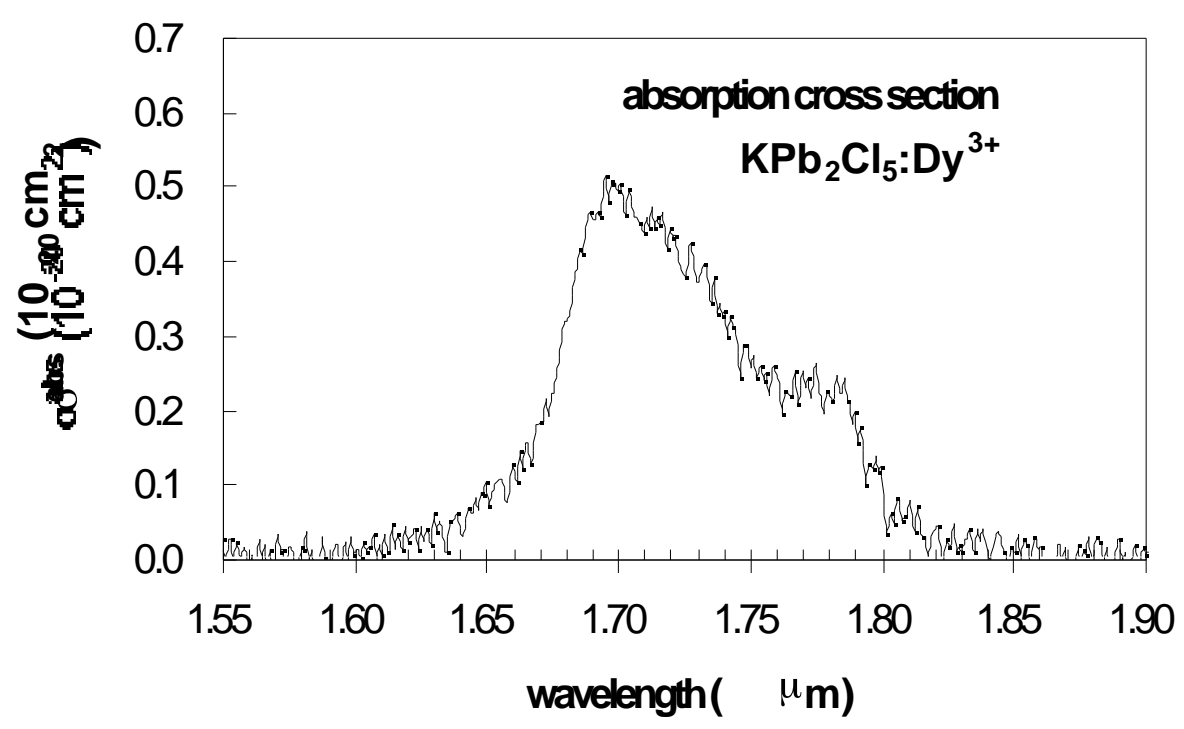

Figure 5.2. Absorption cross section near $1.7 \mu \mathrm{m}$ in $\mathrm{KPb}_{2} \mathrm{Cl}_{5}: \mathrm{Dy}^{3+}$.

With the 4.3- $\mu \mathrm{m}$ laser transition inaccessible to $\mathrm{us}$, the $\mathrm{KPb}_{2} \mathrm{Cl}_{5}: \mathrm{Dy}^{3+} \mathrm{W} \rightarrow \mathrm{Y}$ transition at $2.4 \mu \mathrm{m}$ was downselected for laser experiments since direct population of the upper laser level at $1.3 \mu \mathrm{m}$ is possible. As the lower-level lifetime is much longer than the upper level lifetime, population inversion can not be maintained and roomtemperature $\mathrm{CW}$ operation is not feasible on this transition. A confocal laser cavity geometry was chosen as this is the least sensitive to cavity misalignment, while still providing a small beam waist which leads to lower thresholds.

\subsubsection{Predictions}

Laser threshold and slope efficiency can be predicted based on equations (2.81) and (2.88), respectively. These require the knowledge of the laser waist, pump waist, pump wavelength, emission cross section, pumping efficiency, passive losses, and output coupling. Table 5.1 summarizes the predictions for absorbed threshold and slope for the $\mathrm{KPb}_{2} \mathrm{Cl}_{5}: \mathrm{Dy}^{3+} 2.4 \mu \mathrm{m}$ laser. The laser waist can be calculated from the confocal cavity 
geometry as $w_{0}=\sqrt{\lambda L / 2 \pi}$, where $L$ is the resonator length. The pump spot profile was measured by a razor-scan and fit to a Gaussian ( $1 / \mathrm{e}^{2}$ intensity) radial spot size of $354 \pm 10$ $\mu \mathrm{m}$ (See Appendix G). The emission cross section was determined in Section 4.7 to be $0.50 \times 10^{-20} \mathrm{~cm}^{2}$. The pumping efficiency $\eta_{p, s}$ can be approximated by (2.80). The Boltzmann occupation factors have not been determined. We have estimated however, based on (2.23) and the energy level spectra from $\mathrm{Dy}^{3+}: \mathrm{LaCl}_{3},{ }^{15}$ that $f_{1} \approx f_{2} \approx 10 \%$. The mode overlap efficiency $\eta_{\text {mode }}$ can be estimated from Figure 2.11 (page 2.6-61) for a waist ratio $a \approx 1.3$ and $\mathrm{P}_{\text {in }} / \mathrm{P}_{\text {th }} \approx 4$.

Table 5.1. Values used for the threshold and slope estimates for the $\mathrm{KPb}_{2} \mathrm{Cl}_{5}: \mathrm{Dy}^{3+}$ laser at $2.4 \mu \mathrm{m}$ according to $(2.81)$ and $(2.88)$.

\begin{tabular}{|c|c|c|c|}
\hline$\lambda_{l}$ & 2.4 & $\mu \mathrm{m}$ & laser wavelength \\
\hline$\lambda_{p}$ & 1.319 & $\mu \mathrm{m}$ & pump wavelength \\
\hline$\sigma^{a b s}$ & 2.5 & $10^{-20} \mathrm{~cm}^{2}$ & absorption cross section $\left(\right.$ at $\left.\lambda_{p}\right)$ \\
\hline$w_{l}$ & 276 & $\mu \mathrm{m}$ & laser spot size \\
\hline$w_{p}$ & 354 & $\mu \mathrm{m}$ & pump spot size \\
\hline$\sigma^{e m}$ & 0.5 & $10^{-20} \mathrm{~cm}^{2}$ & emission cross section $\left(\right.$ at $\left.\lambda_{l}\right)$ \\
\hline$L$ & 1.0 & $\%$ & round trip passive losses \\
\hline$T$ & 0.9 & $\%$ & total transmission \\
\hline$\eta_{p, s}$ & 95 & $\%$ & pumping efficiency \\
\hline$f_{2} /\left(f_{l}+f_{2}\right)$ & 50 & $\%$ & bottlenecking factor \\
\hline$\eta_{\text {mode }}$ & 88 & $\%$ & mode-overlap efficiency \\
\hline \hline $\boldsymbol{E}_{\text {th }}$ & $\mathbf{1 . 0}$ & $\mathbf{m J}$ & absorbed-energy threshold \\
\hline$\eta_{\text {slope }}$ & $\mathbf{1 0 . 7}$ & $\%$ & slope efficiency \\
\hline
\end{tabular}

The laser simulation program discussed in Section 2.5 has also been used to predict the laser slope and threshold. In addition to solving the differential equations explicitly, this method is more accurate since it calculates $\eta_{\text {ext }}(r)$ and $\eta_{\text {mode }}(r)$ for each pumping level. Data for $\mathrm{KPb}_{2} \mathrm{Cl}_{5}: \mathrm{Dy}^{3+}$ sample \#1 was used from Table 4.5 for this simulation. A simulated slope-efficiency curve for the $2.4 \mu \mathrm{m}$ laser is shown in Figure 5.3. 


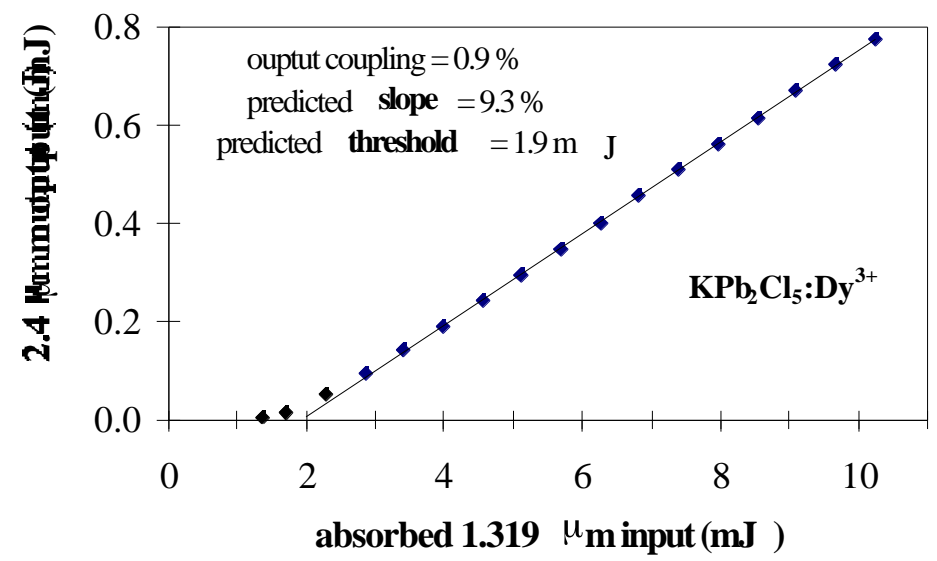

Figure 5.3. Simulated slope-efficiency curve generated from the laser simulation program discussed in Section 2.5.

Notice the threshold prediction here is based on the straight-fit line to the data (as is the actual data), which is greater than the actual threshold since the data is not linear near threshold. The slope prediction is also more meaningful since it too is based on the straight-line fit to the data, which in effect averages out the pump-intensity-dependence of the calculated slope efficiency. A simulated temporal output for $r \equiv E_{i n} / E_{t h}=1.6$ is shown in Figure 5.4 below.

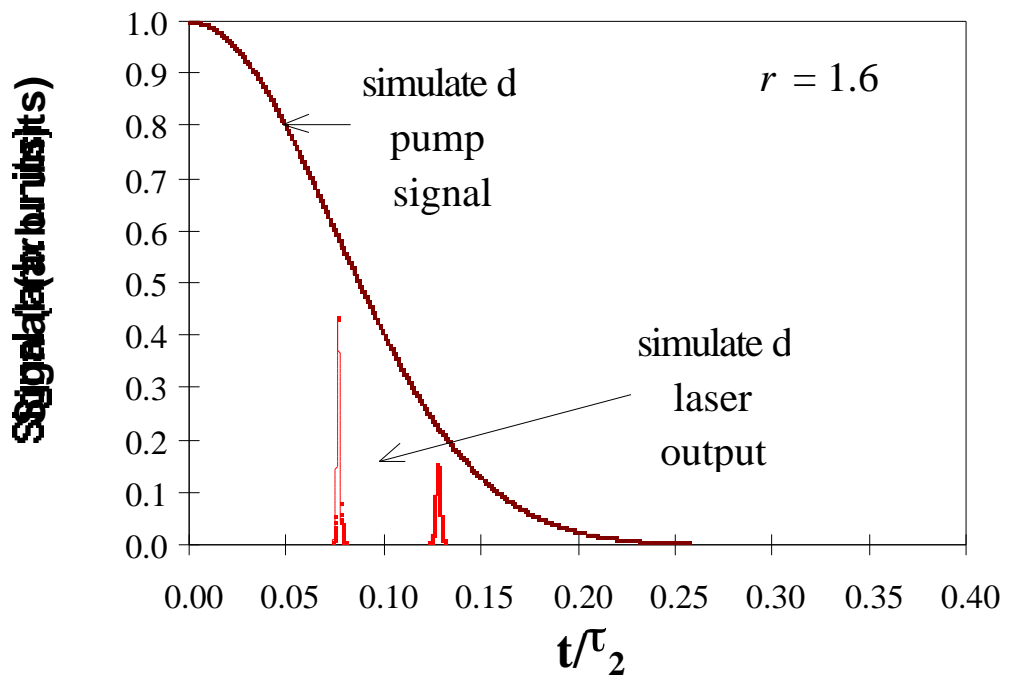

Figure 5.4. Simulated temporal output for $\mathrm{KPb}_{2} \mathrm{Cl}_{5}: \mathrm{Dy}^{3+}$ laser at $2.4 \mu \mathrm{m}$. The pump pulse was modeled as a Gaussian, and the pumping ratio $r=1.6$. The pump and laser signal levels have been arbitrarily adjusted for clarity. 
The pump pulse was modeled as a Gaussian with a FWHM equal to the measured pump pulse. The output shown in Figure 5.4 represents two relaxation oscillations from the laser, which will later be compared to the actual laser output at the same pumping level.

\subsubsection{Experimental Set-up}

Laser experiments were performed on $\mathrm{KPb}_{2} \mathrm{Cl}_{5}: \mathrm{Dy}^{3+}$ (sample \#1). The uncoated $6.4 \mathrm{~mm}$ sample was placed in the center of a $20 \mathrm{~cm}$ confocal cavity and end-pumped at normal incidence ( along an optic axis) by a Nd:YAG laser operating at $1.319 \mu \mathrm{m}$ (and 1.338 $\mu \mathrm{m})$ with $75 \mu \mathrm{s}, 1 \mathrm{~Hz}$ pulses. A $1500 \mathrm{~nm}$ long-pass filter and $10 \mathrm{~cm} \mathrm{CaF}_{2}$ lens imaged the IR laser light at a $77 \mathrm{~K}$ InSb detector for temporal waveform acquisition, or to a Molectron J3-09 pyroelectric detector for slope-efficiency measurements. A glass slide pick-off and Molectron J-25 pyroelectric detector measured the input pump energy.

Figure 5.5 shows a diagram of the set-up.

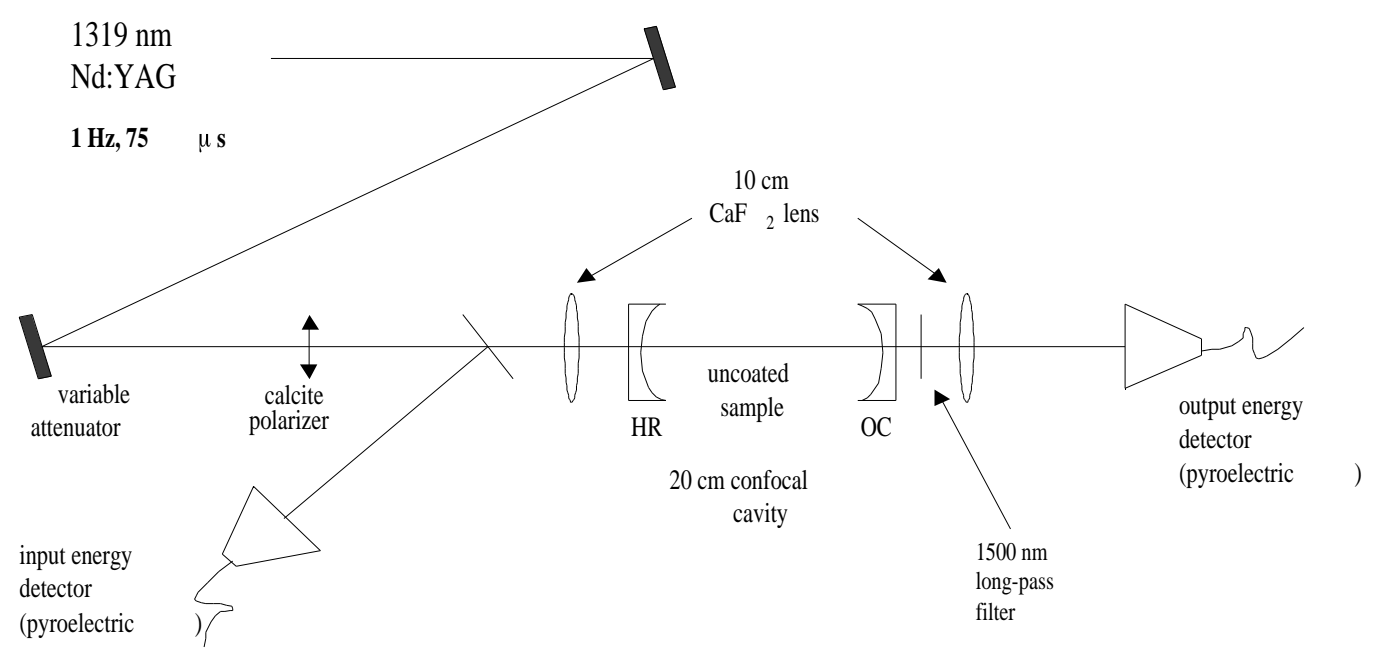

Figure 5.5. Experimental set-up for 1.3- $\mu \mathrm{m}$-pumped laser experiments. 
The laser output spectra were recorded with a 1-meter, 150 groove/mm grating monochromator located $\sim 2$ meters from the cavity. Although the sample had a 'lasergrade' polish, its end faces exhibited a wedge angle of $\sim 2 \mathrm{mrad}$ as determined with a HeNe laser. Four cavity mirrors were available for $2.4 \mu \mathrm{m}$ operation with transmissions of $0.36 \%, 0.58 \%, 1.65 \%$, and $7.70 \%$. Data was recorded for three mirror configurations with total transmissions of $0.94 \%, 2.23 \%$, and $8.28 \%$. The high reflector in each case had a transmission of $0.58 \%$.

\subsubsection{Laser Data and Analysis}

The laser output spectrum is shown in Figure 5.6, along with the unpolarized emission spectrum, showing that laser action occurs at the fluorescence peak. A temporal waveform at pumping ratio $r=1.6$ is shown in Figure 5.7, which compares well to the simulated data in Figure 5.4. The laser light was determined to be unpolarized as expected due to the orientation of the crystal near an optic axis. Slope efficiency curves are shown in Figure 5.8 for two mirror configurations, and all are summarized in Table 5.2. Absorbed energy was limited by concerns of damage to the sample. A damage threshold was estimated to be near $15 \mathrm{~J} / \mathrm{cm}^{2}$ of absorbed energy.

Table 5.2. Measured threshold and slope efficiency for the $\mathrm{KPb}_{2} \mathrm{Cl}_{5}: \mathrm{Dy}^{3+}$ laser at $2.4 \mu \mathrm{m}$.

\begin{tabular}{|c|c|c|}
\hline $\begin{array}{c}\text { Total output } \\
\text { coupling } \\
(\boldsymbol{\%})\end{array}$ & $\begin{array}{c}\text { Absorbed } \\
\text { threshold } \\
(\mathbf{m J})\end{array}$ & $\begin{array}{c}\text { Slope } \\
\text { efficiency } \\
(\boldsymbol{\%})\end{array}$ \\
\hline \hline 0.9 & 1.6 & 0.7 \\
\hline 2.2 & 2.5 & 2.2 \\
\hline 8.3 & 7.2 & 1.7 \\
\hline
\end{tabular}


A Findlay-Clay plot of absorbed energy threshold vs. transmission is shown in Figure 5.9, yielding a round-trip passive loss of $\mathrm{L}=1.2 \% \pm 0.4 \%$. This low value indicates Fresnel reflections imposed by the 2-mrad sample wedge are not completely lost in the confocal cavity geometry. Since modes with lower loss will reach oscillation threshold first, it is logical that the cavity would prefer a mode in which the phase of the Fresnel reflections acted to minimize loss.

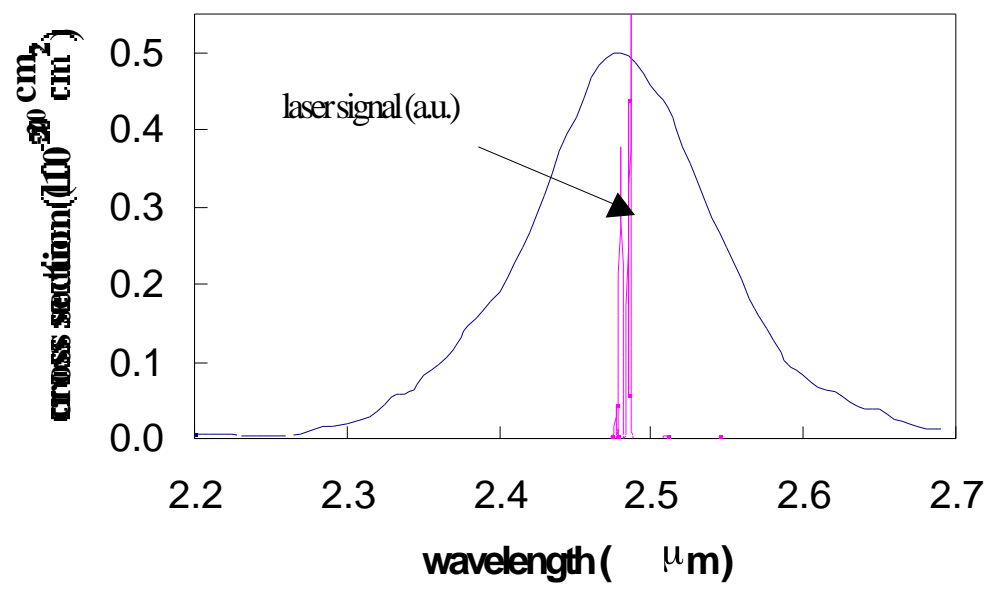

Figure 5.6. Emission spectrum and laser output near $2.4 \mu \mathrm{m}$ in sample \#1.

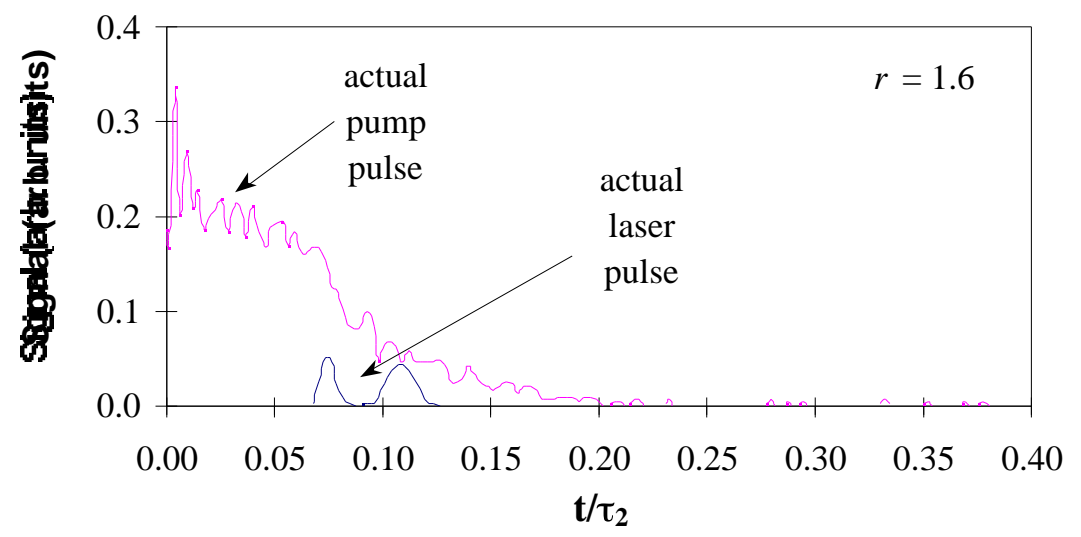

Figure 5.7. Temporal waveform of $\mathrm{KPb}_{2} \mathrm{Cl}_{5}: \mathrm{Dy}^{3+}$ laser at $2.4 \mu \mathrm{m}$. The actual pump pulse is also shown. This can be compared to the predicted waveform from Figure 5.4. 


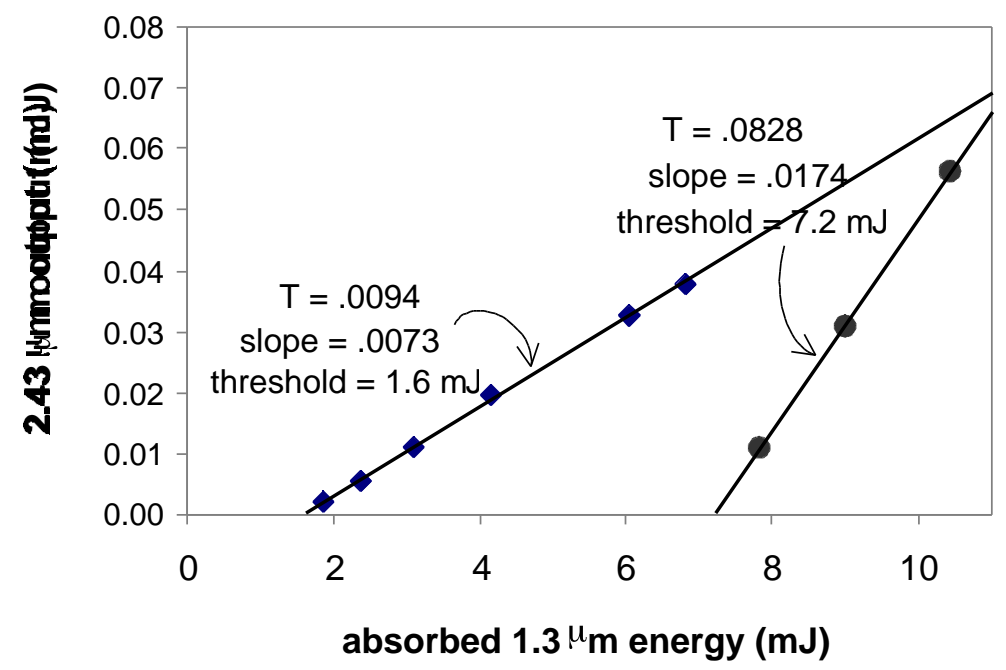

Figure 5.8. Slope efficiency (with respect to absorbed pump energy) curves for $\mathrm{KPb}_{2} \mathrm{Cl}_{5}: \mathrm{Dy}^{3+}$ at $2.43 \mu \mathrm{m}$. T is the total output coupling.

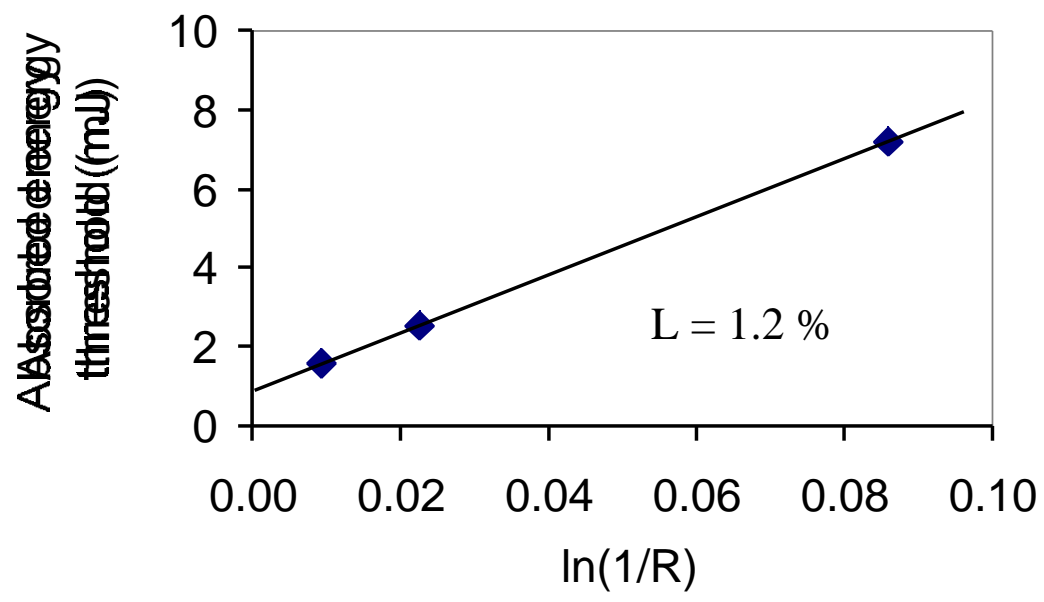

Figure 5.9. "Findlay-Clay" plot of absorbed energy threshold vs. total transmission. A passive loss of $L=1.2 \%$ is inferred from the data.

Referring to the predictions in Table 5.1, we see that the slope efficiency was predicted relatively well (1.0 mJ vs. $1.6 \mathrm{~mJ})$, but the slope efficiencies were overestimated by an order of magnitude. The reason for this discrepancy is not understood. A possible 
explanation (in principle) could be the increased losses due to biaxial "walk-off". "Walkoff" occurs if the $\vec{S}$ and $\vec{k}$ vectors are not parallel, which generally occurs in a biaxial crystal. The case is more easily illustrated for a uniaxial crystal, where only one index forms an ellipsoid (instead of two in the biaxial case). Figure 5.10 depicts the walk-off angle $\delta$ in terms of the incident angle $\theta$. The formula for $\delta$ given by ${ }^{67,68}$

$$
\delta(\theta)=-\tan ^{-1}\left[\left(n_{e} / n_{o}\right)^{2} \tan (\theta)\right]+\theta
$$

where $n_{o}$ and $n_{e}$ are the ordinary and extraordinary indices of refraction, respectively. In our laser experiments, walk-off should have been minimized since the crystal was oriented along a principal axis. A plot of $\delta(\theta)$ vs. $\theta$ is shown in Figure 5.11, indicating that near $\theta=0$ degrees the walk-off angle is minimal for a material such as $\mathrm{KPb}_{2} \mathrm{Cl}_{5}$.

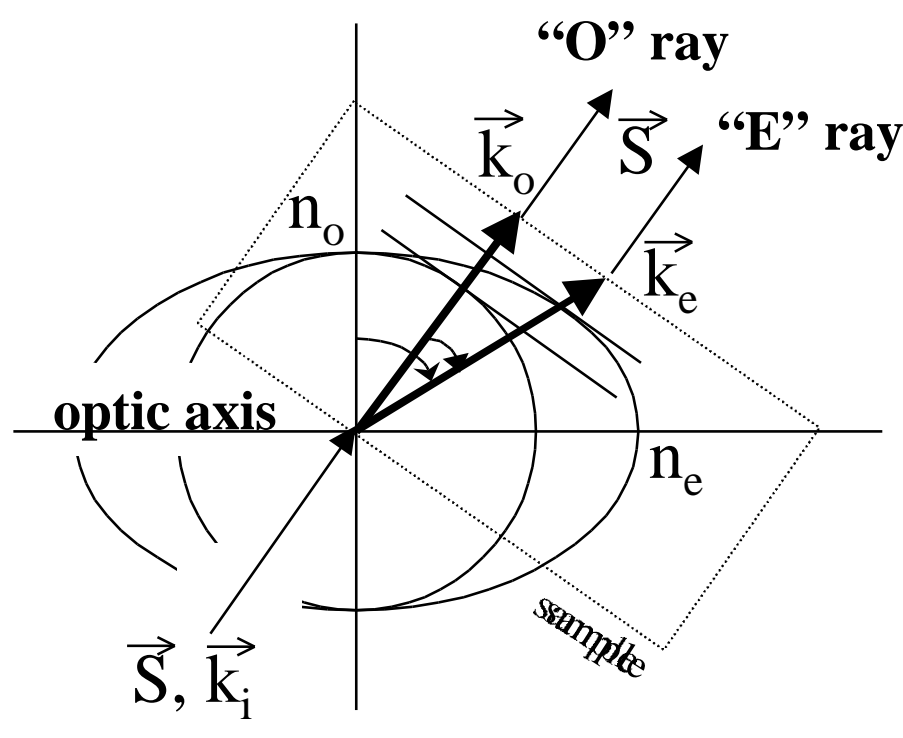

Figure 5.10. "Walk-off" (described by the angle $\delta$ ) occurs when the $S$ and $k$ vectors are not parallel, as is the case when the incident light does not lie along a principal axis. The case shown is for a uniaxial crystal, and $i=$ incident, $o=$ ordinary, $e=$ extraordinary. The ordinary and extraordinary rays exit parallel to the incident ray due to refraction. See Ref. [69] for the details of double refraction in anisotropic crystal. The extraordinary ray is polarized in the plane of the page, the ordinary ray out of the page. 


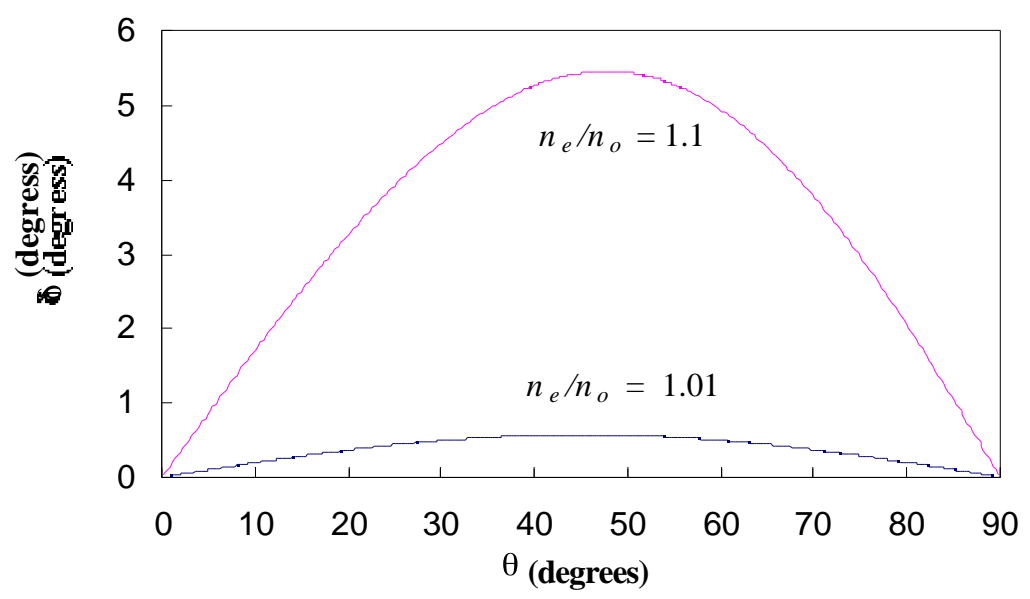

Figure 5.11. Uniaxial walk-off angle $\delta$ vs. incident angle $\theta$ for two values of $n_{e} / n_{o}$. The estimated $\mathrm{KPb}_{2} \mathrm{Cl}_{5}$ index ratio is $n_{e} / n_{o}=1.01$.

\section{$5.2 \mathrm{Nd}^{3+}$ at $1.06 \mu \mathrm{m}$}

Because the $\mathrm{Nd}^{3+} 1.06-\mu \mathrm{m}$ laser transition is well characterized in other hosts (such as YAG), laser experiments were performed with the $\mathrm{KPb}_{2} \mathrm{Cl}_{5}: \mathrm{Nd}^{3+}$ sample on the ${ }^{4} \mathrm{~F}_{3 / 2} \rightarrow{ }^{4} \mathrm{I}_{11 / 2}$ transition at $1.06 \mu \mathrm{m}$. In this way, overall performance of the $\mathrm{KPb}_{2} \mathrm{Cl}_{5}: \mathrm{Nd}^{3+}$ laser could be evaluated with the Nd:YAG laser serving as a benchmark. The pump laser available was a $\mathrm{Cr}^{3+}: \mathrm{LiSAF}$ laser tuned to $0.88 \mu \mathrm{m}$. The laser cavity was $20 \mathrm{~cm}$ in length with mirror curvatures $R_{1}=30 \mathrm{~cm}$ and $R_{2}=40 \mathrm{~cm}$.

\subsubsection{Predictions}

The laser waist, calculated from the cavity geometry, is $275 \mu \mathrm{m}$. The pump spot profile was measured by a razor-scan and fit to a Gaussian (1/e $\mathrm{e}^{2}$ intensity) radial spot size of 490 $\pm 10 \mu \mathrm{m}$ (See Appendix G). The cross section was calculated using (2.20) and the data in Figure 4.22 as $\sigma^{e m}=4.5 \times 10^{-20} \mathrm{~cm}^{2}$. The Boltzmann occupation factors were taken as those for Nd:YAG: ${ }^{48} f_{2}=0.40$ and $f_{1}=0.19$. The mode overlap efficiency $\eta_{\text {mode }}$ can be 
estimated from Figure 2.11 (page 2.6-61) for a waist ratio $a \approx 2.0$ and $r \approx 5$. Table 5.3 summarizes the predictions for absorbed threshold and slope for the $\mathrm{KPb}_{2} \mathrm{Cl}_{5}$ : $\mathrm{Nd}^{3+} 1.06$ $\mu \mathrm{m}$ laser.

Table 5.3. Values used for the threshold and slope estimates for the $\mathrm{KPb}_{2} \mathrm{Cl}_{5}: \mathrm{Nd}^{3+}$ laser at $1.06 \mu \mathrm{m}$ according to (2.81) and (2.88).

\begin{tabular}{|c|c|c|c|}
\hline$\lambda_{l}$ & 1.06 & $\mu \mathrm{m}$ & laser wavelength \\
\hline$\lambda_{p}$ & 0.88 & $\mu \mathrm{m}$ & pump wavelength \\
\hline$w_{l}$ & 248 & $\mu \mathrm{m}$ & laser spot size \\
\hline$w_{p}$ & 490 & $\mu \mathrm{m}$ & pump spot size \\
\hline$\sigma^{e m}$ & 4.5 & $10^{-20} \mathrm{~cm}^{2}$ & emission cross section $\left(\right.$ at $\left.\lambda_{l}\right)$ \\
\hline$L$ & 6.0 & $\%$ & round trip passive loss \\
\hline$T$ & 5.2 & $\%$ & total transmission \\
\hline$\eta_{p, s}$ & 63 & $\%$ & $\begin{array}{c}\text { pumping efficiency for } \\
\text { equivalent square pulse }\end{array}$ \\
\hline$f_{2} /\left(f_{l}+f_{2}\right)$ & 68 & $\%$ & bottlenecking factor \\
\hline$\eta_{\text {mode }}$ & 60 & $\%$ & mode overlap efficiency \\
\hline \hline $\boldsymbol{E}_{\text {th }}$ & $\mathbf{2 . 1}$ & $\mathbf{m J}$ & absorbed energy threshold \\
\hline$\eta_{\text {slope }}$ & $\mathbf{9 . 9}$ & $\%$ & slope efficiency \\
\hline
\end{tabular}

Simulated laser output based on the computer model of Section 2.5 is shown in Figure 5.12 for the two output couplers used $(0.4 \%$ and $5.2 \%)$. This simulation suggests $E_{t h}=$ $3.9 \mathrm{~mJ}$ and $\eta_{\text {slope }}=15.1 \%$. The model also produced a pumping efficiency of $\eta_{p}=63 \%$.

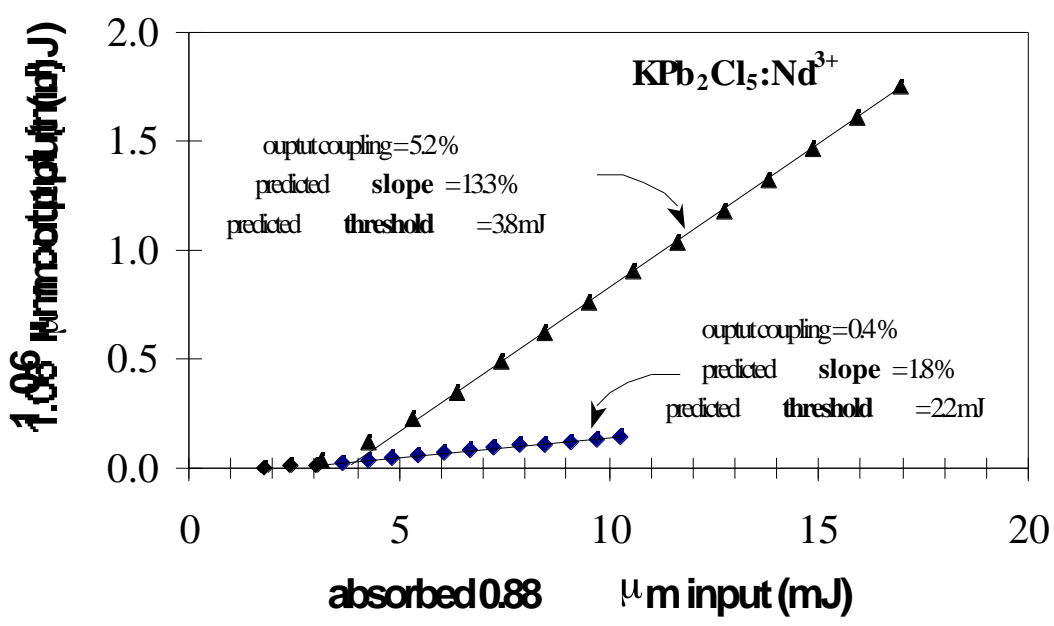

Figure 5.12. Simulated slope-efficiency curve generated from the laser simulation program discussed in Section 2.5 . 


\subsubsection{Experimental Set-up}

The asymmetric cavity used for these experiments consisted of a $20-\mathrm{cm}$ long cavity with a $40-\mathrm{cm}$ radius of curvature mirror on the input side and a $30-\mathrm{cm}$ curvature mirror on the output side. The uncoated 5.5-mm sample was placed $\sim 7 \mathrm{~cm}$ from the output mirror and end-pumped at normal incidence by a $\mathrm{Cr}^{3+}$ :LiSAF laser operating at $0.882 \mu \mathrm{m}$ with 160 $\mu \mathrm{s}, 1 \mathrm{~Hz}$ pulses. A $950 \mathrm{~nm}$ long-pass filter and $10 \mathrm{~cm}$ lens imaged the IR laser light at a Molectron J3-09 pyroelectric detector for slope-efficiency measurements. A glass slide pick-off and Molectron J-25 pyroelectric detector measured the input pump energy. Two output couplers were available for $1.06 \mu \mathrm{m}$ operation with transmissions of $0.2 \%$ and $5.2 \%$. The input mirror was also $0.2 \%$ transmissive at $1.06 \mu \mathrm{m}$. Spectral calibration was verified with a optical spectrum analyzer.

\subsubsection{Laser Data and Analysis}

Threshold and slope efficiency (with respect to absorbed pump power) for the two cavity configurations is shown in Figure 5.13. The threshold prediction agreed well with experiment. The slope efficiencies were also in reasonable agreement, measuring a factor of approximately 1.5 lower than the simulated values. A cross section analysis similar to Section 3.2 produces a value of $\sigma^{e m}=2.1 \times 10^{-20} \mathrm{~cm}^{2}$ for the emission cross section, as compared to the measured value of $\sigma^{e m}=4.5 \times 10^{-20} \mathrm{~cm}^{2}$, confirming the low measured values of the slope efficiencies are not due to unaccounted for passive losses. A FindlayClay analysis estimated the passive losses to be $\mathrm{L} \sim 6 \%$, which is consistent with the 
optical quality of this laser sample (relatively low). The predictable behavior of the $\mathrm{KPb}_{2} \mathrm{Cl}_{5}: \mathrm{Nd}^{3+}$ laser serves to further establish $\mathrm{KPb}_{2} \mathrm{Cl}_{5}$ as a viable laser host.

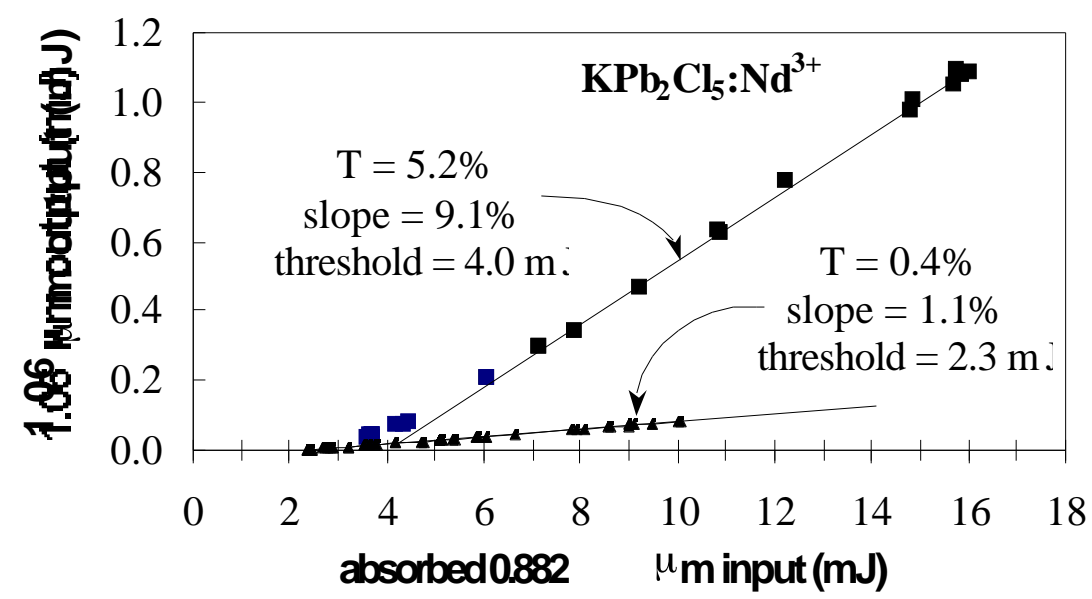

Figure 5.13. Actual laser data collected for $\mathrm{KPb}_{2} \mathrm{Cl}_{5}: \mathrm{Nd}^{3+}$ at $1.06 \mu \mathrm{m}$.

\subsection{Conclusions}

$\mathrm{KPb}_{2} \mathrm{Cl}_{5}$ has proven to be a viable laser host. Laser action at $2.4 \mu \mathrm{m}$ has been demonstrated in $\mathrm{KPb}_{2} \mathrm{Cl}_{5}: \mathrm{Dy}^{3+}$, and at $1.06 \mu \mathrm{m}$ in $\mathrm{KPb}_{2} \mathrm{Cl}_{5}: \mathrm{Nd}^{3+}$. These lasers represent the first room-temperature, rare-earth-doped chloride lasers to operate in ambient conditions. With an appropriate pump source at $1.7 \mu \mathrm{m}$, it is quite clear than $\mathrm{KPb}_{2} \mathrm{Cl}_{5}: \mathrm{Dy}^{3+}$ would support lasing at $4.3 \mu \mathrm{m}$ on the ${ }^{6} \mathrm{H}_{11 / 2} \rightarrow{ }^{6} \mathrm{H}_{13 / 2}$ transition. 


\section{Growth and Characterization of $\mathrm{CaGa}_{2} \mathrm{~S}_{4}: \mathrm{Dy}^{3+}$}

The idea to pursue $\mathrm{CaGa}_{2} \mathrm{~S}_{4}$ came from Dr. William F. Krupke here at LLNL. I believe he found references in the literature about a sulfide compound that was non-hygroscopic and might be easily grown into large single crystals, although no such effort had yet been attempted. A collaboration with Pete Schunemann at Sanders was established since he has expertise in the growth of sulfide crystals (mainly used for nonlinear interactions).

\subsection{Sample Preparation}

The $1.5 \times 1.2 \times 0.7 \mathrm{~cm}^{3}$ sample used in our experiments was cut from a $19 \mathrm{~mm}$-diameter $\mathrm{x}$ $100 \mathrm{~mm}$ single crystal grown by Pete Schunemann using the horizontal gradient freeze technique in a two-zone transparent furnace (growth rate $=0.5-1 \mathrm{~mm} / \mathrm{hr}$ ) ${ }^{70,71}$ A similar sample is shown in Figure 6.1.

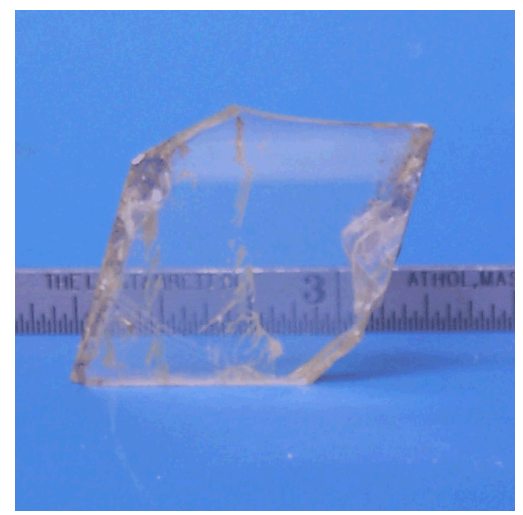

Figure 6.1. $\mathrm{CaGa}_{2} \mathrm{~S}_{4}: \mathrm{Dy}^{3+}$ was grown by the horizontal gradient freeze technique in a two-zone transparent furnace.

The melting point was found to be $1095 \mathrm{C}$. Dy ${ }^{3+}$ was doped to an estimated concentration of $8 \times 10^{19} \mathrm{~cm}^{-3}(2.0 \mathrm{~mol} \%$ in the melt and an estimated $60 \%$ distribution coefficient), presumably on the $\mathrm{Ca}^{2+}$ site. $\mathrm{Na}^{+}$was also added ( $\left.2 \mathrm{~mol} \%\right)$ to maintain 
charge balance. The measured $\mathrm{Na}^{+} / \mathrm{Dy}^{3+}$ concentration ratio was $93 \%$. The compound was pre-synthesized from high-purity starting materials according the reactions $2 \mathrm{Ga}+3 \mathrm{~S}$ $\rightarrow \mathrm{Ga}_{2} \mathrm{~S}_{3}$ (vapor transport) and $\mathrm{CaS}+\mathrm{Ga}_{2} \mathrm{~S}_{3}+\mathrm{Dy}_{2} \mathrm{~S}_{3}+\mathrm{NaS} \bullet \mathrm{Dy}^{3+}, \mathrm{Na}^{+}: \mathrm{CaGa}_{2} \mathrm{~S}_{4}$.

The synthesis and growth were preformed in inert PBN boats sealed in quartz ampoules. The growth apparatus is shown in Figure 6.2.

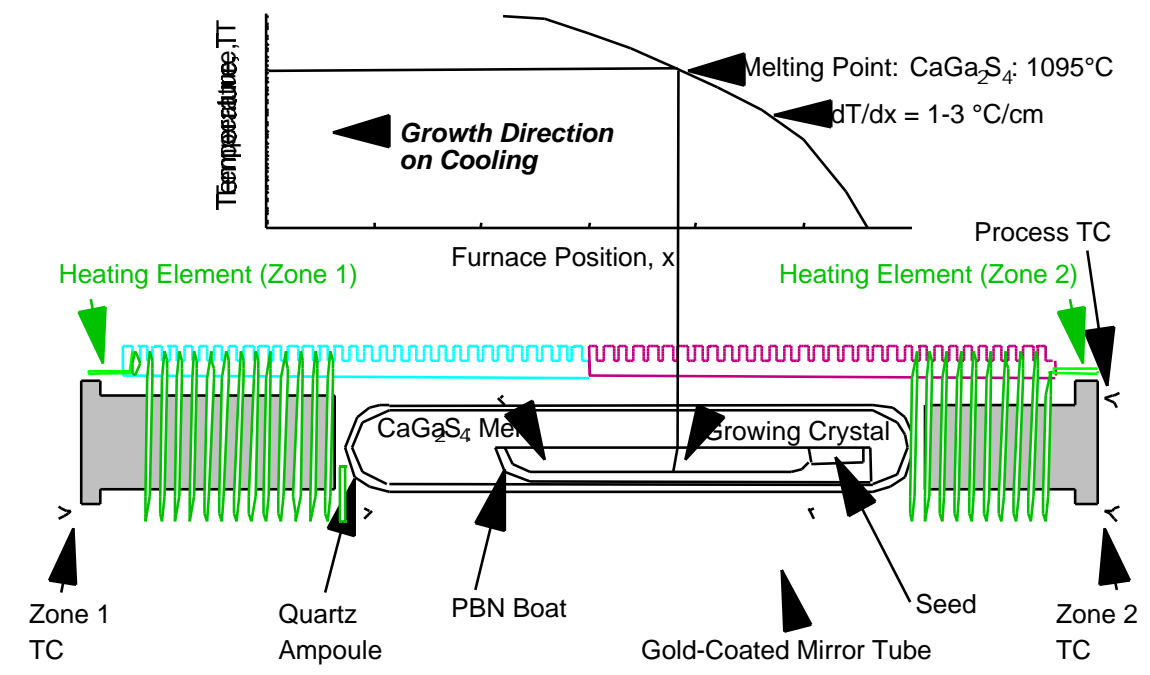

Figure 6.2. Transparent two-zone furnace used to grow crystalline $\mathrm{CaGa}_{2} \mathrm{~S}_{4}: \mathrm{Dy}^{3+}$.

\subsection{Crystal Properties}

$\mathrm{CaGa}_{2} \mathrm{~S}_{4}$ is biaxial, belonging to the orthorhombic crystal class (space group Fddd) with lattice parameters $a=0.2009 \mathrm{~nm}, b=0.2009$, and $c=0.1211 \mathrm{~nm}$. The divalent calcium cations occupy square antiprismatic sites between sheets of $\mathrm{GaS}_{4}$ tetrahedra joined by corner- or edge-sharing. ${ }^{72}$ This sheet structure makes $\mathrm{CaGa}_{2} \mathrm{~S}_{4}$ behave much like mica, a common mineral than can easily be cleaved into thin sheets. $\mathrm{Ca}^{2+}$ is eight-fold coordinated, $\mathrm{S}^{2-}$ and $\mathrm{Ga}^{3+}$ are both four-fold coordinated. A maximum phonon frequency 
of $\sim 350 \mathrm{~cm}^{-1}$ is reported for $\mathrm{CaGa}_{2} \mathrm{~S}_{4} \cdot{ }^{73}$ This is consistent with the measured IR cutoff wavelength of $\sim 12 \mu \mathrm{m}$ shown in Figure 6.3 (See Appendix E, page 185).

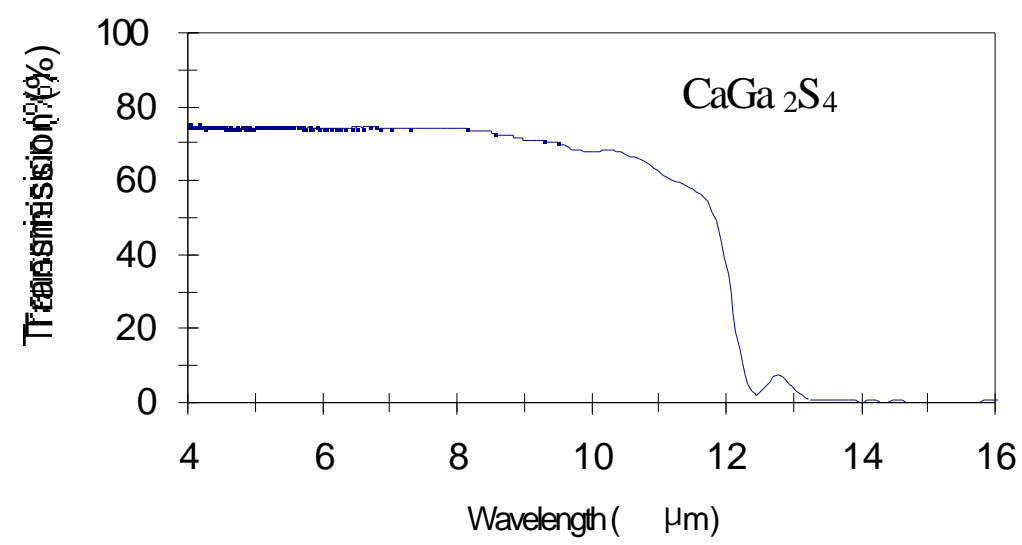

Figure 6.3. IR transmission of $\mathrm{CaGa}_{2} \mathrm{~S}_{4}$. Sample thickness was $2.5 \mathrm{~mm}$.

Indices of refraction were estimated based on the analysis of Appendix D ("Refractive index based on transmission measurements", page 183). Two orthogonal directions were identified in a thin, cleaved sheet of material at extinction positions between crossed polarizers. Transmission spectra were recorded polarized along each direction, and the refractive index was calculated for each wavelength according to (9.23). The results are shown in Figure 6.4.

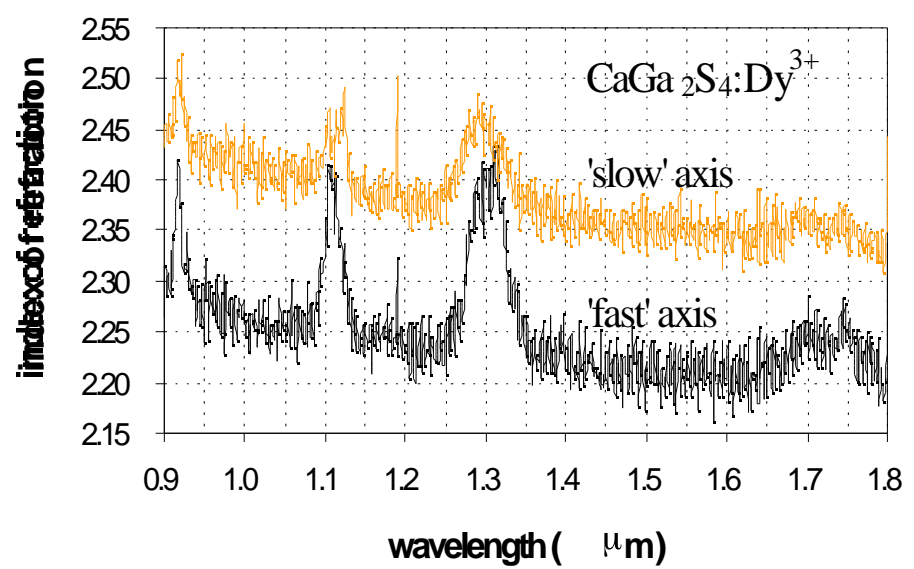

Figure 6.4. Index of refraction for $\mathrm{CaGa}_{2} \mathrm{~S}_{4}: \mathrm{Dy}^{3+}$ was calculated using (9.23) along two orthogonal polarization axes. The axis associated with the smaller index is labeled the 'fast' axis, the other is the 'slow' axis. The peaks correspond the $\mathrm{Dy}^{3+}$ absorptions. The average $\Delta \mathrm{n}$ is $\sim 0.15$. 
Near $1.0 \mu \mathrm{m}$, these results indicate $n_{\text {slow }} \approx 2.40$ and $n_{\text {fast }} \approx 2.25$. Unpolarized spectra indicate an average index of $n=2.3$. These data indicate an average $\Delta n$ of 0.15 , which is relatively quite large. For comparison, the $\Delta n$ of other common materials are: quartz: 0.009, beryl: 0.008 , mica: 0.036 , topaz: 0.008 , gypsum: 0.010 , feldspar: 0.008 , calcite: 0.172. Thus $\mathrm{CaGa}_{2} \mathrm{~S}_{4}$ is similar in $\Delta n$ to calcite, which is a material commonly found in polarizers. Thus $\mathrm{CaGa}_{2} \mathrm{~S}_{4}$ may be well-suited as a mid-IR polarizer. The physics behind the large $\Delta n$ probably lies in the "sheet-like" structure of $\mathrm{CaGa}_{2} \mathrm{~S}_{4}$, see Figure 6.5. The atoms in the plane of a sheet (the cleavage plane) are coupled together much differently (stronger) than those between sheets. Therefore the vibrations in these two directions are much different, which leads to different polarizabilities and indices in these different directions.

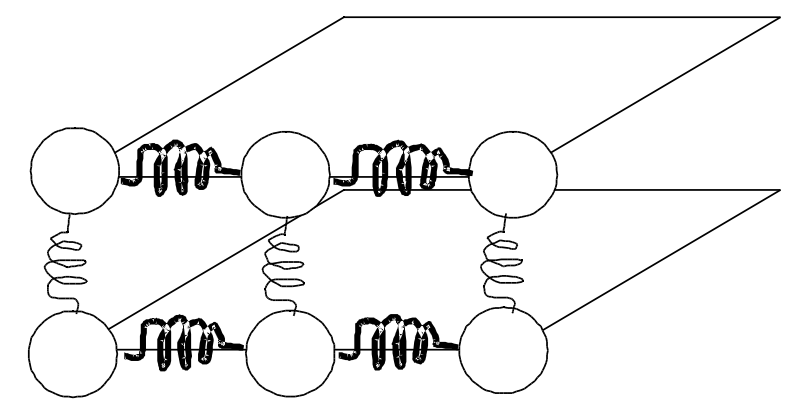

Figure 6.5. An oversimplified view of atoms within and between cleavage planes. The atoms in a cleavage plane are coupled together much differently than those between planes, leading to a large index of refraction difference in the two directions.

\subsection{Absorption Spectra}

Unpolarized absorption spectra taken at room-temperature $(300 \mathrm{~K})$ and cold-temperature $(15 \mathrm{~K})$ are shown in Figure 6.6. Absorption cross section was determined from the $\mathrm{Dy}^{3+}$ 
concentration $N_{0}$ and absorption coefficient $\alpha$ via $\sigma=N_{0^{*}} \alpha . \quad N_{O}$ was determined by Theresa Duewer here at LLNL with the use of an inductively-coupled plasma mass spectrometer $\mathrm{Dy}^{3+}$ absorption bands are clearly identifiable (c.f. Figure 3.1). The 2.85 $\mu \mathrm{m} Y$-level peak coincides with a $2.9 \mu \mathrm{m} \mathrm{H}_{2} \mathrm{O}$ feature, identified with an asterisk in the figure. Unlike that for $\mathrm{KPb}_{2} \mathrm{Cl}_{5}: \mathrm{Dy}^{3+}$, the $1.3-\mu \mathrm{m}$ feature is not hypersensitive (in the sense that the feature is not abnormally strong). The $15 \mathrm{~K}$ spectrum shows line narrowing due to the thermal depopulation of the 16 levels comprising the ${ }^{6} \mathrm{H}_{15 / 2}$ ground state.

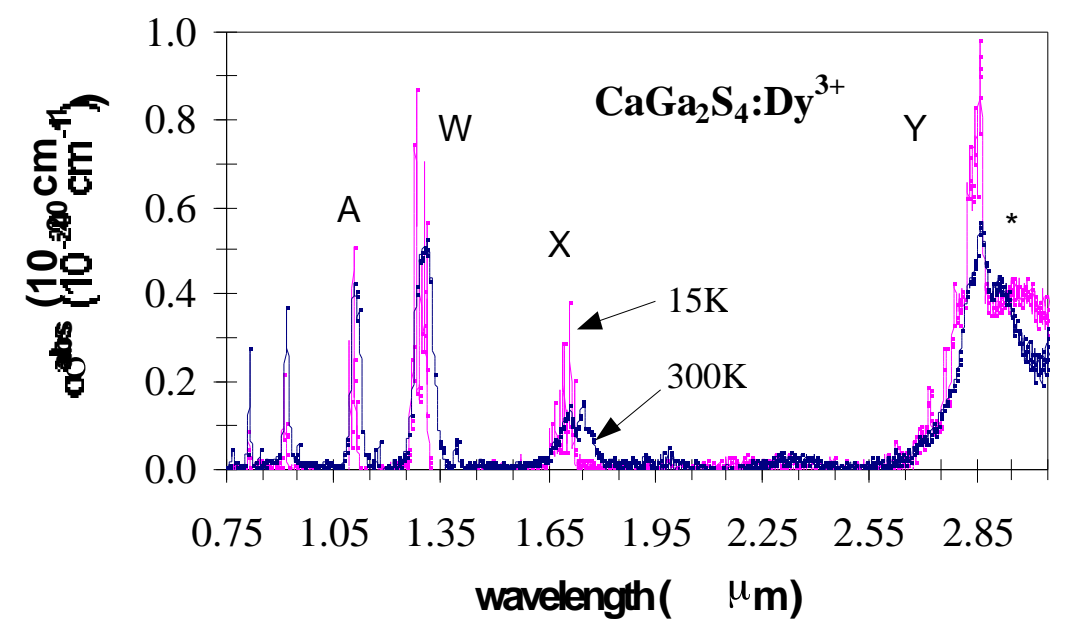

Figure 6.6. Absorption cross section of $\mathrm{CaGa}_{2} \mathrm{~S}_{4}: \mathrm{Dy}^{3+}$ at $15 \mathrm{~K}$ and $300 \mathrm{~K}$.

Polarized spectra has also been recorded for $\mathrm{CaGa}_{2} \mathrm{~S}_{4}: \mathrm{Dy}^{3+}$. The data for the 'fast' and 'slow' axis (see Section 6.2) is shown in Figure 6.7. An important feature of this crystal is the appearance of small absorption peaks red-shifted from the main peaks at 0.9, 1.1, and $1.3 \mu \mathrm{m}$. They become bright emission features, supporting lasing near $1.4 \mu \mathrm{m}$. These appear to be due to Stark splitting by the crystal field, with one stark level lying $\sim 400 \mathrm{~cm}^{-1}$ above the ground state. Another dysprosium-doped crystal, YLF, also exhibits this property. ${ }^{74}$ Experimentally measured energy levels for $\mathrm{Dy}^{3+}$ :YLF are shown in Figure 6.8. 


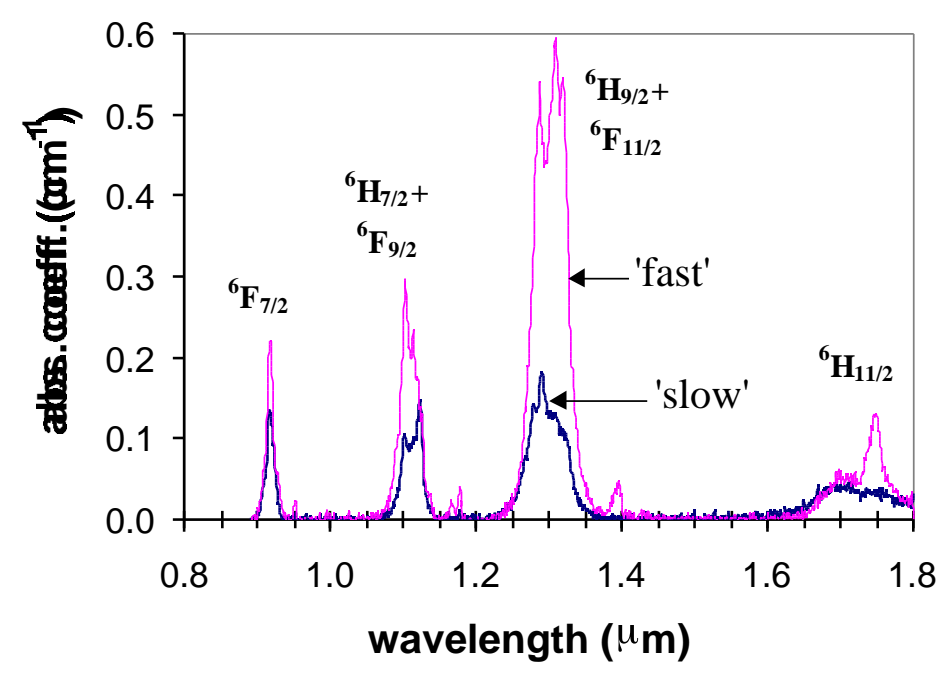

Figure 6.7. Polarized absorption spectrum of $\mathrm{CaGa}_{2} \mathrm{~S}_{4}: \mathrm{Dy}^{3+}$. Notice the absorption is nearly 5 times greater along the 'fast' axis near $1.3 \mu \mathrm{m}$. Only two of the three principal axes of the biaxial indicatrix were accessible to absorption measurements.

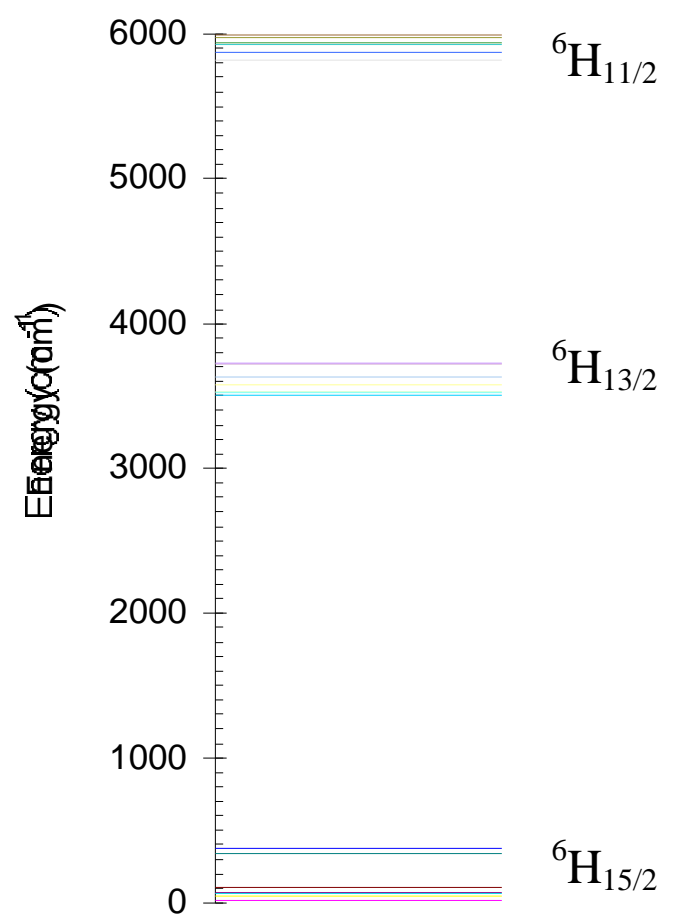

Figure 6.8. Experimentally determined energy levels in Dy:YLF (after Ref. 74) 


\subsection{Judd-Ofelt Data}

A Judd-Ofelt analysis was performed using the unpolarized absorption spectra according to the developments in Section 2.4. Table 6.1 gives the results of this analysis. The three Judd-Ofelt parameters were determined to be: $\Omega_{2}=3.78 \times 10^{-20} \mathrm{~cm}^{2}, \Omega_{4}=2.02 \times 10^{-20}$ $\mathrm{cm}^{2}, \Omega_{6}=1.94 \times 10^{-20} \mathrm{~cm}^{2}$. Notice the magnetic dipole contributions for the low-lying $|\Delta J|=1$ states are comparable to the electric dipole contributions.

Table 6.1. Results of the Judd-Ofelt analysis for $\mathrm{CaGa}_{2} \mathrm{~S}_{4}: \mathrm{Dy}^{3+}$.

\begin{tabular}{|c|c|c|c|c|c|c|c|}
\hline Transition & $\begin{array}{c}\lambda \\
(\mu \mathbf{m})\end{array}$ & $\begin{array}{c}S^{\mathrm{ED}} \\
\left(10^{-20} \mathrm{~cm}^{2}\right)\end{array}$ & $\begin{array}{c}\mathrm{S}^{\mathrm{MD}} \\
\left(10^{-20} \mathrm{~cm}^{2}\right)\end{array}$ & $\begin{array}{l}\mathrm{A}^{\mathrm{ED}} \\
\left(\mathrm{s}^{-1}\right)\end{array}$ & $\begin{array}{l}\mathrm{A}^{\mathrm{MD}} \\
\left(\mathrm{s}^{-1}\right)\end{array}$ & $\beta^{\mathrm{rad}}$ & $\begin{array}{c}\tau_{\mathrm{rad}} \\
(\mathbf{m s})\end{array}$ \\
\hline${ }^{6} \mathbf{H}_{13 / 2} \rightarrow{ }^{6} \mathbf{H}_{15 / 2}$ & 2.82 & 2.91 & 1.02 & 91.4 & 28.8 & 1.000 & 8.32 \\
\hline${ }^{6} \mathrm{H}_{11 / 2} \rightarrow{ }^{6} \mathrm{H}_{13 / 2}$ & 4.23 & 1.89 & 1.49 & 20.5 & 14.4 & 0.122 & \multirow{2}{*}{3.50} \\
\hline$\rightarrow{ }^{6} \mathrm{H}_{15 / 2}$ & 1.69 & 1.48 & 0.00 & 250.7 & 0.0 & 0.878 & \\
\hline${ }^{6} \mathrm{H}_{9 / 2} \rightarrow{ }^{6} \mathrm{H}_{11 / 2}$ & 5.57 & 1.60 & 1.64 & 9.1 & 8.3 & 0.060 & \multirow{3}{*}{3.46} \\
\hline$\rightarrow{ }^{6} \mathrm{H}_{13 / 2}$ & 2.40 & 1.17 & 0.00 & 82.7 & 0.0 & 0.286 & \\
\hline$\rightarrow{ }^{6} \mathrm{H}_{15 / 2}$ & 1.30 & 0.42 & 0.00 & 189.3 & 0.0 & 0.654 & \\
\hline${ }^{6} \mathbf{F}_{11 / 2} \rightarrow{ }^{6} \mathbf{H}_{9 / 2}$ & - & 1.11 & 0.05 & 0.0 & 0.0 & 0.000 & \multirow{4}{*}{0.45} \\
\hline$\rightarrow{ }^{6} \mathrm{H}_{11 / 2}$ & 5.57 & 1.92 & 0.03 & 9.1 & 0.1 & 0.004 & \\
\hline$\rightarrow{ }^{6} \mathrm{H}_{13 / 2}$ & 2.40 & 3.14 & 0.00 & 185.5 & 0.0 & 0.084 & \\
\hline$\rightarrow{ }^{6} \mathrm{H}_{15 / 2}$ & 1.30 & 5.37 & 0.00 & 2014.0 & 0.0 & 0.912 & \\
\hline${ }^{6} \mathbf{H}_{7 / 2} \rightarrow{ }^{6} \mathbf{F}_{11 / 2}$ & 7.60 & 0.32 & 0.00 & 0.9 & 0.0 & 0.004 & \multirow{5}{*}{4.23} \\
\hline$\rightarrow^{6} \mathrm{H}_{9 / 2}$ & 7.60 & 1.46 & 1.50 & 4.1 & 3.8 & 0.033 & \\
\hline$\rightarrow{ }^{6} \mathrm{H}_{11 / 2}$ & 3.21 & 1.00 & 0.00 & 37.1 & 0.0 & 0.157 & \\
\hline$\rightarrow{ }^{6} \mathrm{H}_{13 / 2}$ & 1.83 & 0.58 & 0.00 & 116.8 & 0.0 & 0.494 & \\
\hline$\rightarrow{ }^{6} \mathrm{H}_{15 / 2}$ & 1.11 & 0.08 & 0.00 & 74.0 & 0.0 & 0.313 & \\
\hline${ }^{6} \mathbf{F}_{9 / 2} \rightarrow{ }^{6} \mathbf{H}_{7 / 2}$ & - & 1.04 & 0.00 & 0.0 & 0.0 & 0.000 & \multirow{6}{*}{0.43} \\
\hline$\rightarrow{ }^{6} \mathrm{~F}_{11 / 2}$ & 7.60 & 0.11 & 0.54 & 0.3 & 1.1 & 0.001 & \\
\hline$\rightarrow^{6} \mathrm{H}_{9 / 2}$ & 7.60 & 1.12 & 0.00 & 2.5 & 0.0 & 0.001 & \\
\hline$\rightarrow{ }^{6} \mathrm{H}_{11 / 2}$ & 3.21 & 1.66 & 0.01 & 49.2 & 0.4 & 0.021 & \\
\hline$\rightarrow^{6} \mathrm{H}_{13 / 2}$ & 1.83 & 3.13 & 0.00 & 505.4 & 0.0 & 0.217 & \\
\hline$\rightarrow{ }^{6} \mathrm{H}_{15 / 2}$ & 1.11 & 2.45 & 0.00 & 1766.7 & 0.0 & 0.760 & \\
\hline${ }^{6} \mathbf{H}_{5 / 2} \rightarrow{ }^{6} \mathbf{F}_{9 / 2}$ & 10.1 & 0.65 & 0.00 & 1.1 & 0.0 & 0.007 & \multirow{7}{*}{6.48} \\
\hline$\rightarrow^{6} \mathrm{H}_{7 / 2}$ & 10.1 & 1.30 & 0.95 & 2.1 & 1.4 & 0.023 & \\
\hline$\rightarrow{ }^{6} \mathrm{~F}_{11 / 2}$ & 4.33 & 0.04 & 0.00 & 0.8 & 0.0 & 0.005 & \\
\hline$\rightarrow{ }^{6} \mathrm{H}_{9 / 2}$ & 4.33 & 0.93 & 0.00 & 18.8 & 0.0 & 0.121 & \\
\hline$\rightarrow{ }^{6} \mathrm{H}_{11 / 2}$ & 2.44 & 0.59 & 0.00 & 67.0 & 0.0 & 0.434 & \\
\hline$\rightarrow{ }^{6} \mathrm{H}_{13 / 2}$ & 1.55 & 0.12 & 0.00 & 53.1 & 0.0 & 0.344 & \\
\hline$\rightarrow{ }^{6} \mathrm{H}_{15 / 2}$ & 1.00 & 0.01 & 0.00 & 10.2 & 0.0 & 0.066 & \\
\hline
\end{tabular}




\subsection{Emission Data}

Emission cross sections of the $\mathrm{CaGa}_{2} \mathrm{~S}_{4}: \mathrm{Dy}^{3+}$ transitions can be calculated (via (2.20)) using the branching ratios and radiative lifetimes found in Section 6.4. Spectrally calibrated emission spectra were taken with a computer controlled 1-meter scanning monochromator. The sample (2.0 mol\% doping) was directly excited by a CW source and a reference chopper was placed at the entrance slit of the monochromator to modulate the emission signal at $77 \mathrm{~Hz}$. The W-level was pumped with a $1.319 \mu \mathrm{m}, 100 \mathrm{~mW}$ Nd:YAG laser, while the $\mathrm{X}$-level was pumped with a $\sim 1 \mathrm{~W}$ CW InGaAsP-InP diode source at 1.7 $\mu \mathrm{m}$. The $1.3-\mu \mathrm{m}$ emission lineshape was obtained by pumping with $\sim 150 \mathrm{~mW}$ of 0.81 $\mu \mathrm{m}$ output from a $\mathrm{Ti}^{3+}: \mathrm{Al}_{2} \mathrm{O}_{3}$ laser. Calibrated emission data for $\mathrm{CaGa}_{2} \mathrm{~S}_{4}: \mathrm{Dy}^{3+}$ is shown in Figure 6.9. Emission cross section values will be shown in the next section.

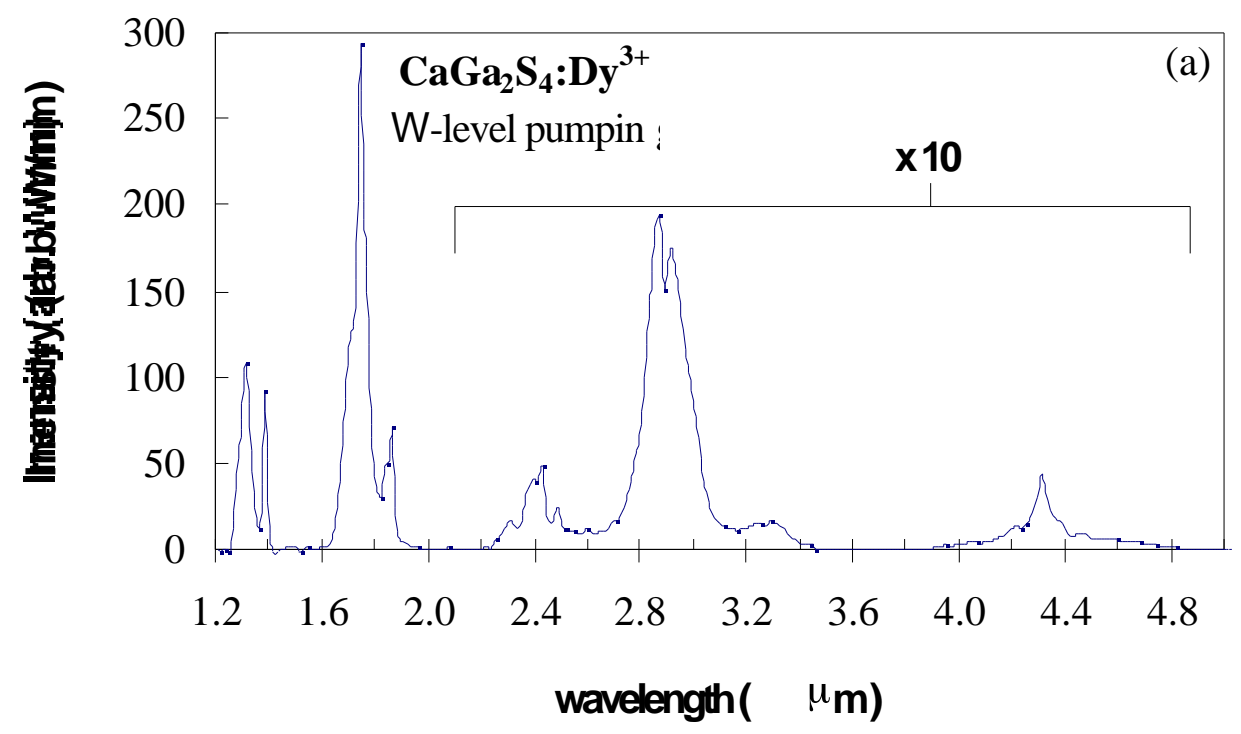




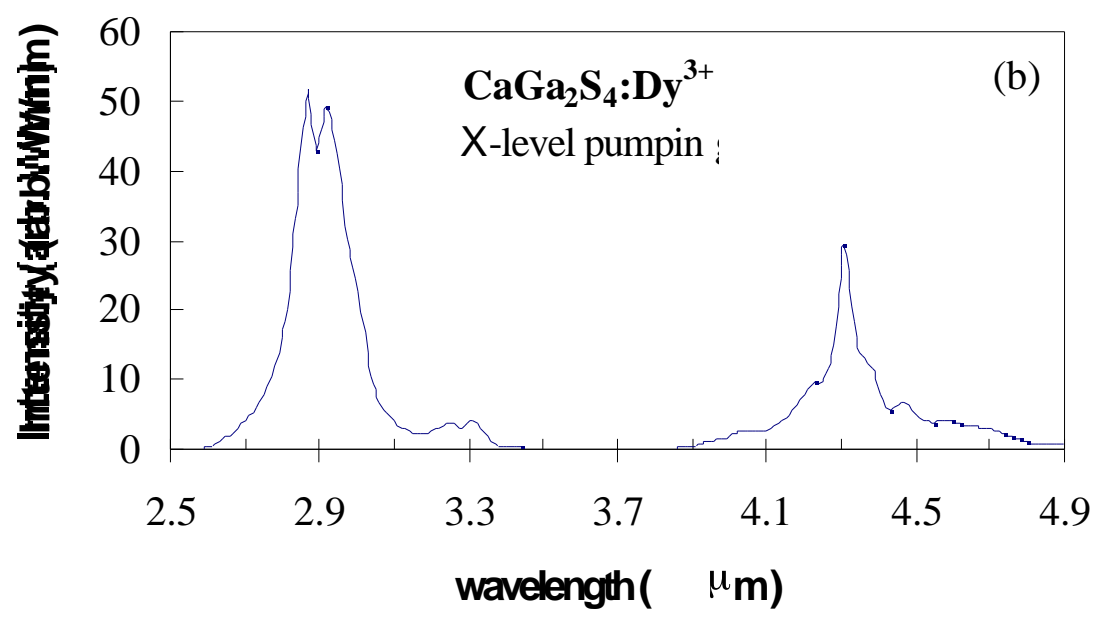

Figure 6.9. Calibrated emission spectra for $\mathrm{CaGa}_{2} \mathrm{~S}_{4}: \mathrm{Dy}^{3+}$. (a) full spectrum upon $\mathrm{W}$-level pumping. (b) partial spectrum upon $\mathrm{X}$-level pumping $(1.7 \mu \mathrm{m}$ peak not shown).

\subsection{Quantum Efficiencies}

The calibrated emission data from Figure 6.9 was used to determine radiative lifetimes and quantum efficiencies according to the model described in Section 3.1. The results are shown below in Table 6.2, along with the corresponding Judd-Ofelt values for comparison.

Table 6.2. Measured branching ratios, radiative lifetimes, and radiative quantum efficiencies for $\mathrm{CaGa}_{2} \mathrm{~S}_{4}: \mathrm{Dy}^{3+}$ are compared to Judd-Ofelt derived data from Table 6.1. The measured total lifetimes and peak emission cross sections (calculated via (2.20) ) are also indicated.

\begin{tabular}{|c|c|c|c|c|c|c|c|c|c|c|}
\hline \multirow[b]{2}{*}{ Transition } & \multirow[b]{2}{*}{$\lambda_{\text {peak }}$} & \multirow[b]{2}{*}{$\tau^{\text {meas }}$} & \multicolumn{4}{|c|}{ Judd-Ofelt Calc'n } & \multicolumn{4}{|c|}{ Measured Data } \\
\hline & & & $\beta^{\mathrm{rad}}$ & $\tau^{\mathrm{rad}}$ & $\eta^{\mathrm{rad}}$ & $\sigma_{\text {em,peak }}$ & $\beta^{\mathrm{rad}}$ & $\tau^{\mathrm{rad}}$ & $\eta^{\mathrm{rad}}$ & $\sigma_{\text {em,peak }}$ \\
\hline & $(\mu \mathbf{m})$ & (ms) & & (ms) & & $\left(10^{-20} \mathrm{~cm}^{2}\right)$ & & $(\mathrm{ms})$ & & $\left(10^{-20} \mathrm{~cm}^{2}\right)$ \\
\hline $\mathrm{W} \rightarrow \mathrm{X}$ & 5.5 & \multirow{3}{*}{0.20} & 0.010 & \multirow{3}{*}{0.75} & \multirow{3}{*}{0.27} & $\mathrm{n} / \mathrm{a}$ & 0.005 & \multirow{3}{*}{1.00} & \multirow{3}{*}{0.20} & $\mathrm{n} / \mathrm{a}$ \\
\hline $\mathrm{W} \rightarrow \mathrm{Y}$ & 2.4 & & 0.104 & & & 1.22 & 0.134 & & & 1.18 \\
\hline $\mathrm{W} \rightarrow \mathrm{Z}$ & 1.3 & & 0.886 & & & 1.88 & 0.861 & & & 1.37 \\
\hline$X \rightarrow Y$ & 4.3 & \multirow{2}{*}{3.02} & 0.122 & \multirow{2}{*}{3.50} & \multirow{2}{*}{0.86} & 2.29 & 0.077 & \multirow{2}{*}{3.09} & \multirow{2}{*}{0.98} & 1.64 \\
\hline$X \rightarrow Z$ & 1.7 & & 0.878 & & & 1.02 & 0.923 & & & 1.21 \\
\hline$Y \rightarrow Z$ & 2.8 & 7.85 & 1.000 & 8.32 & 0.94 & 1.29 & 1.000 & 7.85 & 1.00 & 1.37 \\
\hline
\end{tabular}


These data assume a unit quantum efficiency for the $\mathrm{Y}$-level since multiphonon decay would involve a $10^{\text {th }}$ order process. This also assumes other impurities, such as $\mathrm{OH}^{-}$, do not contribute to the decay. Evidence for such impurities has not been observed.

\subsection{Other Chalcogenide Samples}

In addition to $\mathrm{CaGa}_{2} \mathrm{~S}_{4}$, other chalcogenide samples were investigated as potential mid-IR laser host materials. These included crystalline $\mathrm{CaGa}_{2} \mathrm{Se}_{4}, \mathrm{GaLaS}$ glass, GeGaS glass, and GeGaAsS glass. In the following sections, we briefly summarize some basic results for these hosts.

\subsection{1 $\mathrm{CaGa}_{2} \mathrm{Se}_{4}: \mathrm{Dy}^{3+}$}

With concentrated growth efforts, this low-phonon-frequency host could serve extremely well as a long-wavelength host material. Its IR cutoff wavelength, as shown in Figure 6.10 , is $\sim 500 \mathrm{~cm}^{-1}$, implying an effective phonon energy of $\sim 250 \mathrm{~cm}^{-1}$, similar to that of $\mathrm{KPb}_{2} \mathrm{Cl}_{5}$. The $\sim 68 \%$ transmission from $4-12 \mu \mathrm{m}$ implies a refractive index of $\sim 2.5$ according the graph in Appendix D. Only one growth effort was attempted (at Sanders), with marginal success. The optical quality was fair, but good enough to obtain absorption and emission data. 


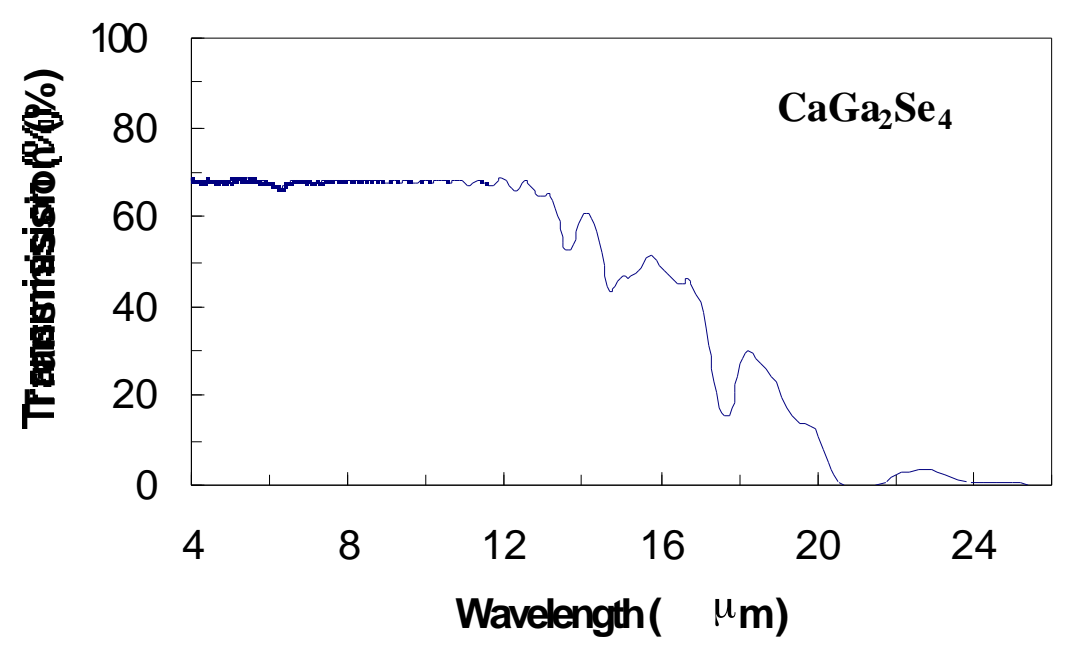

Figure 6.10. Absorption spectrum showing the IR-cutoff of $\mathrm{CaGa}_{2} \mathrm{Se}_{4}$. The features at 13.5, 15, and $17.5 \mu \mathrm{m}$ are unassigned.

The absorption spectrum of $\mathrm{CaGa}_{2} \mathrm{Se}_{4}: \mathrm{Dy}^{3+}$ is shown in Figure 6.11, indicating good incorporation of the $\mathrm{Dy}^{3+}$ ion. The positions, widths, and relative intensities of the peaks are very similar to the $\mathrm{CaGa}_{2} \mathrm{~S}_{4}: \mathrm{Dy}^{3+}$ spectrum. Notice also that there is no evidence of $\mathrm{OH}^{-}$contamination near $3 \mu \mathrm{m}$. Total emission lifetimes were measured for the $\mathrm{A}, \mathrm{W}, \mathrm{X}$ and $\mathrm{Y}$ levels as $0.25,0.51,2.42$, and $5.02 \mathrm{~ms}$, respectively. Compared to $\mathrm{CaGa}_{2} \mathrm{~S}_{4}: \mathrm{Dy}^{3+}$, the longer W level lifetime and the larger A-level emission are indicative of the lower phonon energy of $\mathrm{CaGa}_{2} \mathrm{Se}_{4}$. The emission spectrum is shown in Figure 6.12. Assuming (as in the case of $\mathrm{KPb}_{2} \mathrm{Cl}_{5}: \mathrm{Dy}^{3+}$ ) that the radiative quantum efficiencies of the $\mathrm{X}$ and $\mathrm{Y}$ level are unity, the radiative quantum efficiency of the W-level can be determined via (3.16). 


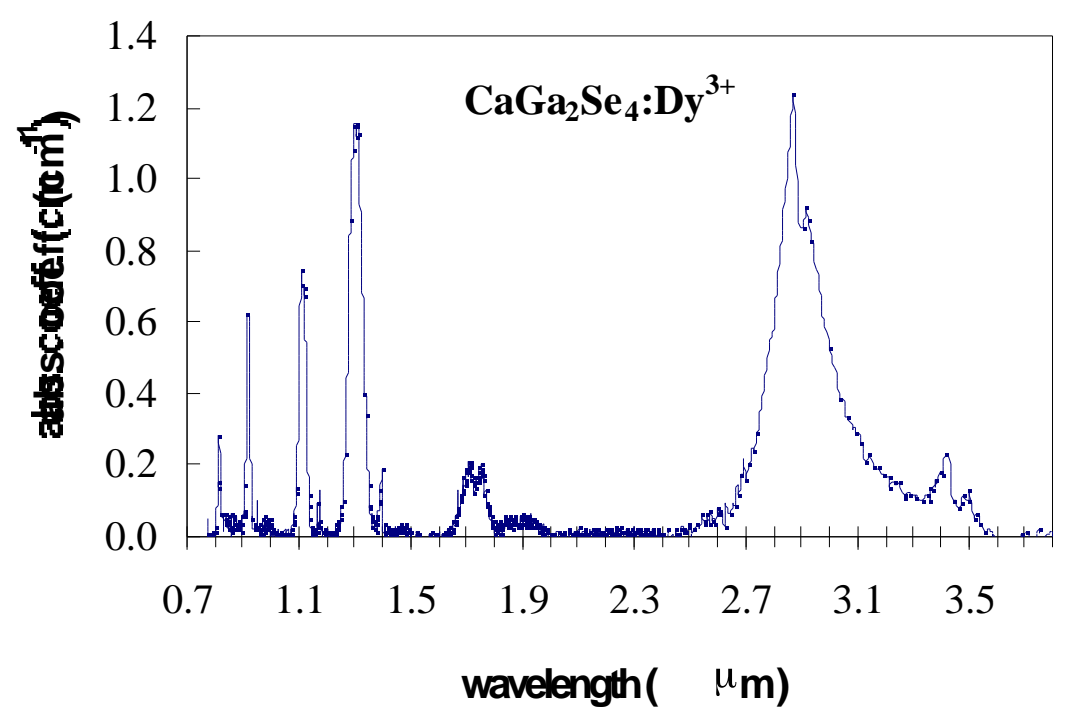

Figure 6.11. Absorption spectrum of $\mathrm{CaGa}_{2} \mathrm{Se}_{4}: \mathrm{Dy}^{3+}$. The spectrum is similar to $\mathrm{CaGa}_{2} \mathrm{~S}_{4}: \mathrm{Dy}^{3+}$ with the small red-shifted peaks and a non-hypersensitive $1.3 \mu \mathrm{m}$ peak.

This gives $\eta_{\mathrm{w}}^{\text {rad }}=0.96 \pm 0.02$ (noting that the relative size of the $2.9 \mu \mathrm{m}$ peak suggests the cross-relaxation in this material is negligible). Similarly, we can use (3.17) to estimate $\eta_{A}^{r a d}=0.10 \pm 0.05$. These high efficiencies suggest the effective phonon energy of $\mathrm{CaGa}_{2} \mathrm{Se}_{4}$ may be even lower than that of $\mathrm{KPb}_{2} \mathrm{Cl}_{5}$. 

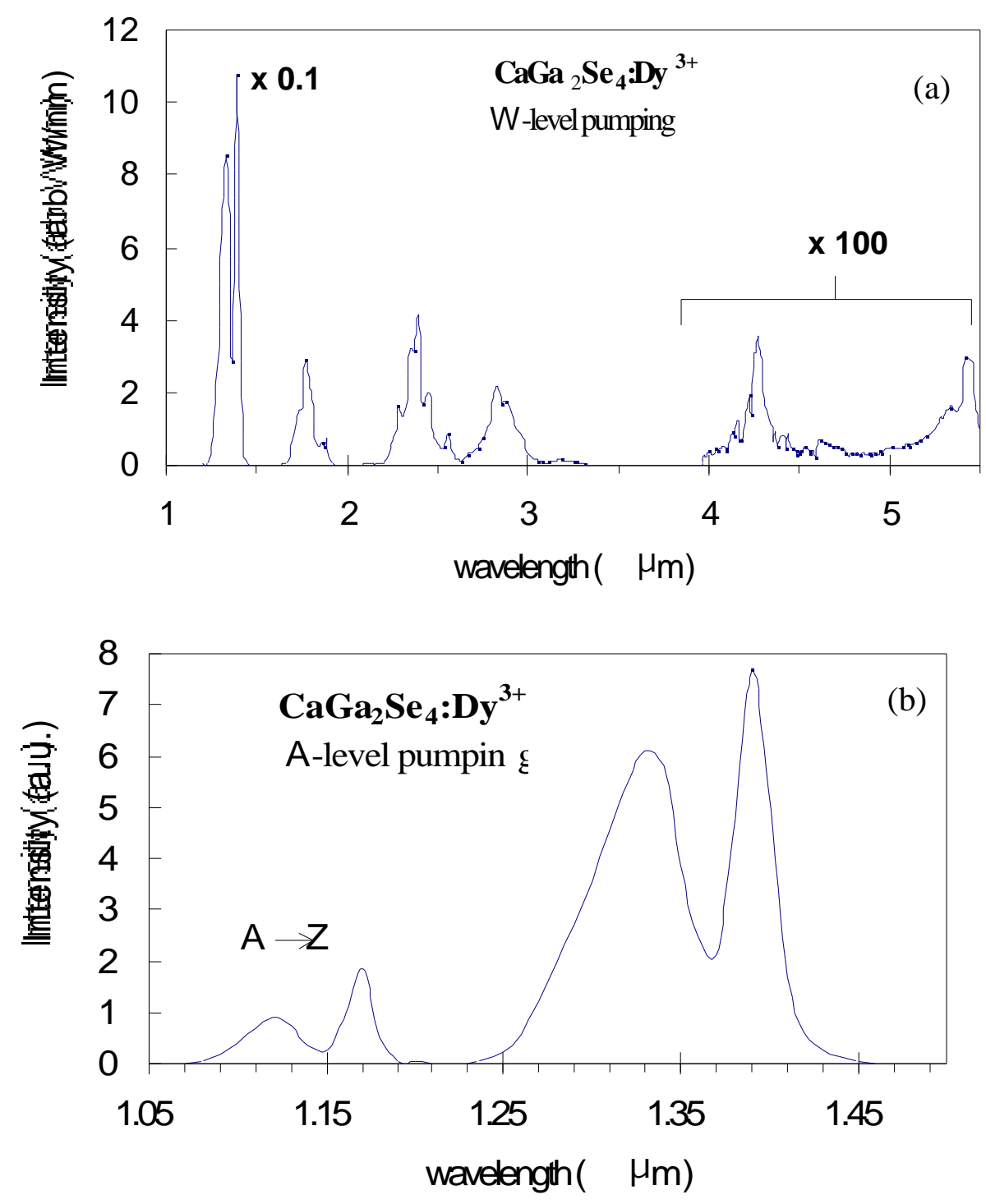

Figure 6.12. (a) Calibrated emission spectrum of $\mathrm{CaGa}_{2} \mathrm{Se}_{4}: \mathrm{Dy}^{3+}$ upon $1.3 \mu \mathrm{m}$ pumping. Note the scale changes indicated on the figure. The output is limited to 5.5 $\mu \mathrm{m}$ due to the cutoff of the InSb detector. (b) uncalibrated emission spectrum upon $0.9 \mu \mathrm{m}$ pumping. Note the emission from the A level. The larger red-shifted peaks are due to transitions to the Stark level lying $400 \mathrm{~cm}^{-1}$ above the ground state.

\subsubsection{GeGaAsS:Dy ${ }^{3+}$ glass}

This sample was furnished by E. Snitzer at Rutgers University. The optical quality of the sample was very good. This sample, however, did not produce laser action near $4-\mu \mathrm{m}$ 
after repeated attempts. Typically, small damage sites would be created, sometimes at the surface but more often in the bulk. The reason might be illustrated in the IR absorption spectrum, shown in Figure 6.13, where impurity absorptions near $4 \mu \mathrm{m}$ are visible. These impurities most likely created additional loss at the laser wavelength, thereby raising the laser threshold above the damage threshold.

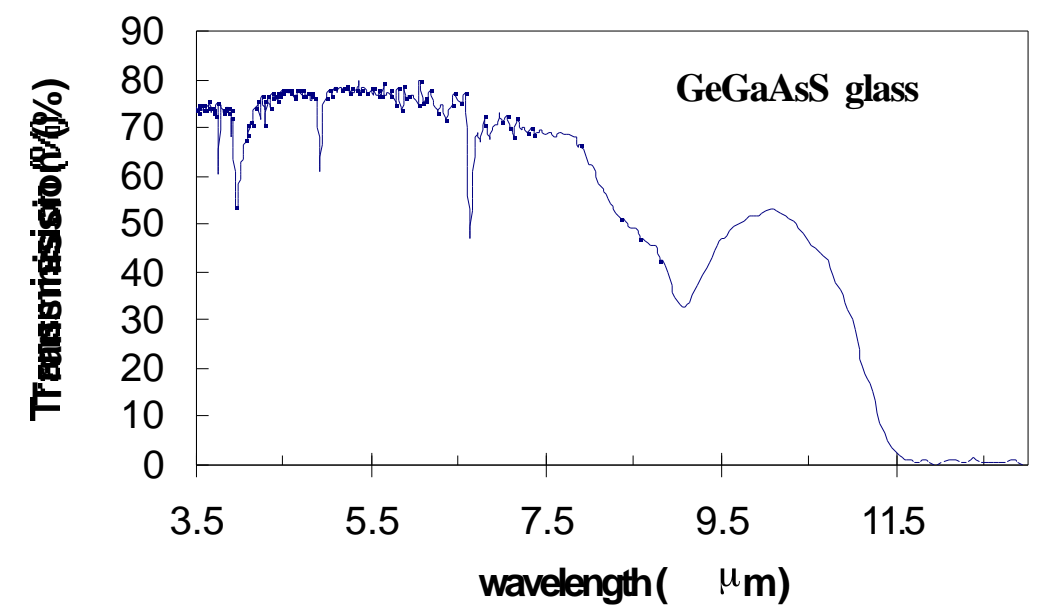

Figure 6.13. IR transmission spectrum of GeGaAsS glass. Unassigned impurity absorption features near 4.0, 4.9, 6.6, and $9.0 \mu \mathrm{m}$ are observed.

The absorption spectrum of GeGaAsS:Dy ${ }^{3+}$ (Figure 6.14) shows the hypersensitive nature of the $1.3-\mu \mathrm{m}$ peak (note its strength relative to the features around it), as well as the broader, smoother absorption features typical of ions in glassy hosts.

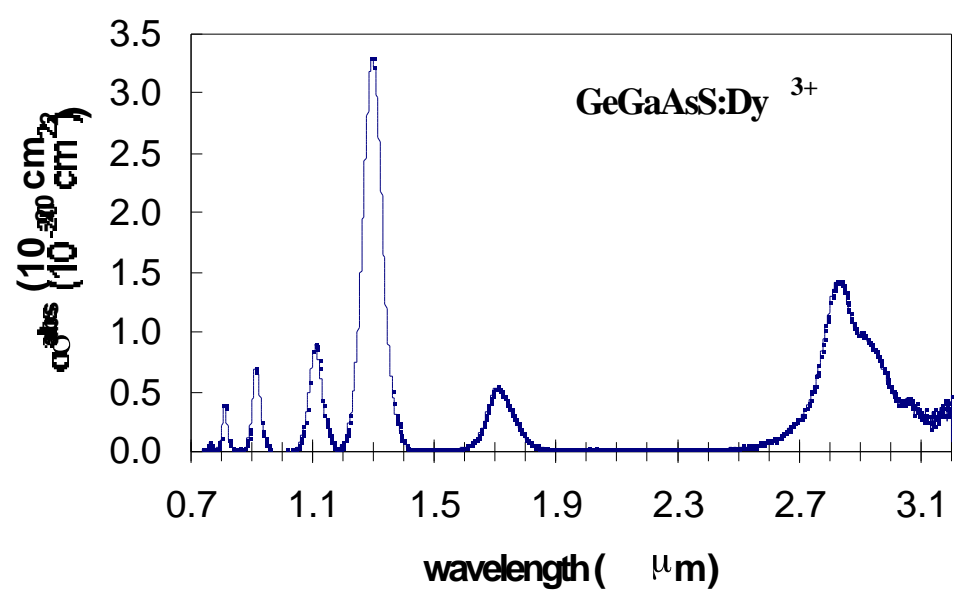

Figure 6.14. Absorption cross section for GeGaAsS:Dy ${ }^{3+}$ glass. 
A Judd-Ofelt analysis was performed on this sample. The Judd-Ofelt parameters (with the hypersensitive $1.3 \mu \mathrm{m}$ transition omitted) were found to be: $\Omega_{2}=13.78 \times 10^{-20} \mathrm{~cm}^{2}$, $\Omega_{4}=2.18 \times 10^{-20} \mathrm{~cm}^{2}, \Omega_{6}=1.96 \times 10^{-20} \mathrm{~cm}^{2}$. A summary of the results is given in Table 6.3.

Table 6.3. Results of the Judd-Ofelt analysis for GeGaAsS:Dy ${ }^{3+}$.

\begin{tabular}{|c|c|c|c|c|c|c|c|}
\hline Transition & $\begin{array}{c}\lambda \\
(\mu \mathbf{m})\end{array}$ & $\begin{array}{c}\mathrm{S}^{\mathrm{ED}} \\
\left(10^{-20} \mathrm{~cm}^{2}\right)\end{array}$ & $\begin{array}{c}\mathrm{S}^{\mathrm{MD}} \\
\left(10^{-20} \mathrm{~cm}^{2}\right)\end{array}$ & $\begin{array}{l}\mathrm{A}^{\mathrm{ED}} \\
\left(\mathrm{s}^{-1}\right) \\
\end{array}$ & $\begin{array}{l}\mathrm{A}^{\mathrm{MD}} \\
\left(\mathbf{s}^{-1}\right)\end{array}$ & $\beta^{\mathrm{rad}}$ & $\begin{array}{c}\tau_{\mathrm{rad}} \\
(\mathbf{m s})\end{array}$ \\
\hline 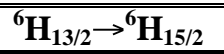 & 2.82 & 5.27 & 1.02 & 195.7 & 32.7 & 1.000 & 4.38 \\
\hline${ }^{6} \mathrm{H}_{11 / 2} \rightarrow{ }^{6} \mathrm{H}_{13 / 2}$ & 4.23 & 4.43 & 1.49 & 56.7 & 16.4 & 0.144 & \multirow{2}{*}{1.97} \\
\hline$\rightarrow{ }^{6} \mathrm{H}_{15 / 2}$ & 1.69 & 2.17 & 0.00 & 434.8 & 0.0 & 0.856 & \\
\hline${ }^{6} \mathrm{H}_{9 / 2} \rightarrow{ }^{6} \mathrm{H}_{11 / 2}$ & 5.57 & 4.91 & 1.64 & 33.0 & 9.5 & 0.108 & \multirow{3}{*}{2.54} \\
\hline$\rightarrow{ }^{6} \mathrm{H}_{13 / 2}$ & 2.40 & 1.48 & 0.00 & 123.7 & 0.0 & 0.314 & \\
\hline$\rightarrow{ }^{6} \mathrm{H}_{15 / 2}$ & 1.30 & 0.43 & 0.00 & 227.3 & 0.0 & 0.578 & \\
\hline${ }^{6} \mathrm{~F}_{11 / 2} \rightarrow{ }^{6} \mathrm{H}_{9 / 2}$ & - & 1.18 & 0.05 & 0.0 & 0.0 & 0.000 & \multirow{4}{*}{0.15} \\
\hline$\rightarrow{ }^{6} \mathrm{H}_{11 / 2}$ & 5.57 & 1.98 & 0.03 & 11.1 & 0.1 & 0.002 & \\
\hline$\rightarrow{ }^{6} \mathrm{H}_{13 / 2}$ & 2.40 & 5.50 & 0.00 & 383.8 & 0.0 & 0.057 & \\
\hline$\rightarrow{ }^{6} \mathrm{H}_{15 / 2}$ & 1.30 & 14.25 & 0.00 & 6324.7 & 0.0 & 0.941 & \\
\hline${ }^{6} \mathbf{H}_{7 / 2} \rightarrow{ }^{6} \mathbf{F}_{11 / 2}$ & 7.60 & 0.33 & 0.00 & 1.1 & 0.0 & 0.004 & \multirow{5}{*}{3.25} \\
\hline$\rightarrow{ }^{6} \mathrm{H}_{9 / 2}$ & 7.60 & 4.46 & 1.50 & 14.7 & 4.3 & 0.062 & \\
\hline$\rightarrow{ }^{6} \mathrm{H}_{11 / 2}$ & 3.21 & 1.34 & 0.00 & 58.7 & 0.0 & 0.191 & \\
\hline$\rightarrow{ }^{6} \mathrm{H}_{13 / 2}$ & 1.83 & 0.59 & 0.00 & 140.4 & 0.0 & 0.456 & \\
\hline$\rightarrow{ }^{6} \mathrm{H}_{15 / 2}$ & 1.11 & 0.08 & 0.00 & 88.5 & 0.0 & 0.288 & \\
\hline${ }^{6} \mathbf{F}_{9 / 2} \rightarrow{ }^{6} \mathbf{H}_{7 / 2}$ & - & 1.10 & 0.00 & 0.0 & 0.0 & 0.000 & \multirow{6}{*}{0.24} \\
\hline$\rightarrow{ }^{6} \mathrm{~F}_{11 / 2}$ & 7.60 & 0.26 & 0.54 & 0.7 & 1.2 & 0.000 & \\
\hline$\rightarrow{ }^{6} \mathrm{H}_{9 / 2}$ & 7.60 & 1.53 & 0.00 & 4.1 & 0.0 & 0.001 & \\
\hline$\rightarrow{ }^{6} \mathrm{H}_{11 / 2}$ & 3.21 & 4.10 & 0.01 & 143.6 & 0.4 & 0.035 & \\
\hline$\rightarrow{ }^{6} \mathrm{H}_{13 / 2}$ & 1.83 & 9.34 & 0.00 & 1782.5 & 0.0 & 0.434 & \\
\hline$\rightarrow{ }^{6} \mathrm{H}_{15 / 2}$ & 1.11 & 2.55 & 0.00 & 2177.3 & 0.0 & 0.530 & \\
\hline${ }^{6} \mathbf{H}_{5 / 2} \rightarrow{ }^{6} \mathbf{F}_{9 / 2}$ & 10.1 & 0.66 & 0.00 & 1.3 & 0.0 & 0.006 & \multirow{7}{*}{5.14} \\
\hline$\rightarrow{ }^{6} \mathrm{H}_{7 / 2}$ & 10.1 & 3.38 & 0.95 & 6.5 & 1.6 & 0.041 & \\
\hline$\rightarrow{ }^{6} \mathrm{~F}_{11 / 2}$ & 4.33 & 0.04 & 0.00 & 1.0 & 0.0 & 0.005 & \\
\hline$\rightarrow{ }^{6} \mathrm{H}_{9 / 2}$ & 4.33 & 1.18 & 0.00 & 28.3 & 0.0 & 0.145 & \\
\hline$\rightarrow{ }^{6} \mathrm{H}_{11 / 2}$ & 2.44 & 0.60 & 0.00 & 80.5 & 0.0 & 0.413 & \\
\hline$\rightarrow{ }^{6} \mathrm{H}_{13 / 2}$ & 1.55 & 0.12 & 0.00 & 63.5 & 0.0 & 0.326 & \\
\hline$\rightarrow{ }^{6} \mathrm{H}_{15 / 2}$ & 1.00 & 0.01 & 0.00 & 12.2 & 0.0 & 0.063 & \\
\hline
\end{tabular}


In order to compare with the other crystalline materials, a quantum efficiency analysis (see Section 3.1) was performed on this sample. The calibrated emission spectrum used for this analysis is shown in Figure 6.15. The experimental conditions were identical to those for $\mathrm{CaGa}_{2} \mathrm{~S}_{4}: \mathrm{Dy}^{3+}$.
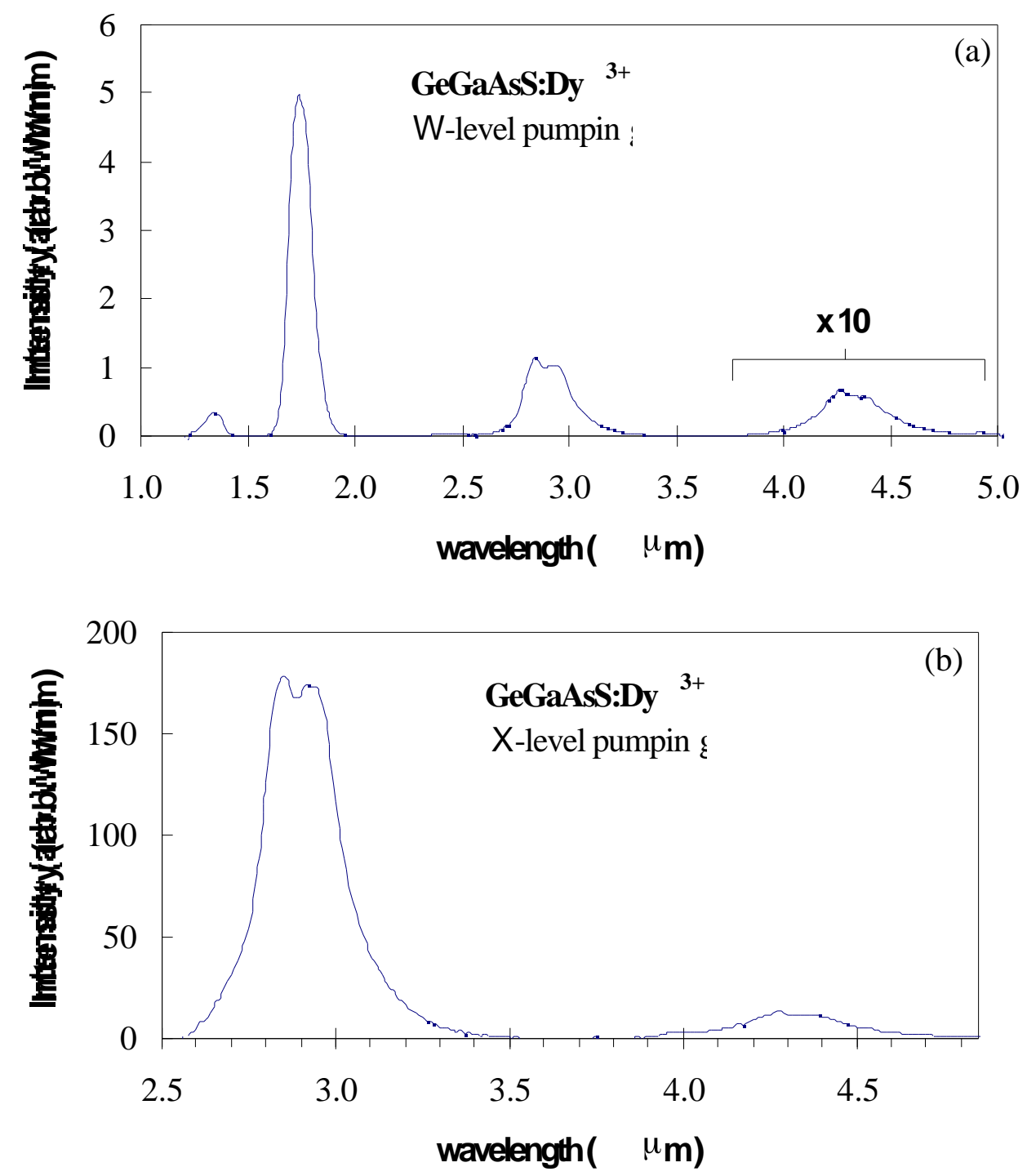

Figure 6.15. Calibrated emission spectrum of GaGeAsS:Dy ${ }^{3+}$ under (a) W-level pumping and (b) $\mathrm{X}$-level pumping.

The experimentally determined radiative lifetimes and branching ratios are shown in Table 6.4, along with the corresponding Judd-Ofelt derived data. The two methods are in 
reasonable agreement. Were it not for the impurity absorptions near $4 \mu \mathrm{m}$, this host would be an excellent candidate for a $4 \mu \mathrm{m}$ laser.

Table 6.4. Measured branching ratios, radiative lifetimes, and radiative quantum efficiencies for GeGaAsS:Dy ${ }^{3+}$ are compared to Judd-Ofelt derived data from Table 6.3. The measured total lifetimes and peak emission cross sections (calculated via (2.20) ) are also indicated.

\begin{tabular}{|c|c|c|c|c|c|c|c|c|c|c|}
\hline \multirow[b]{2}{*}{ Transition } & \multirow[b]{2}{*}{$\lambda_{\text {peak }}$} & \multirow[b]{2}{*}{$\tau^{\text {meas }}$} & \multicolumn{4}{|c|}{ Judd-Ofelt Calc'n } & \multicolumn{4}{|c|}{ Measured Data } \\
\hline & & & $\beta^{\mathrm{rad}}$ & $\tau^{\mathrm{rad}}$ & $\eta^{\mathrm{rad}}$ & $\sigma_{\text {em,peak }}$ & $\beta^{\mathrm{rad}}$ & $\tau^{\mathrm{rad}}$ & $\eta^{\mathrm{rad}}$ & $\sigma_{\text {em,peak }}$ \\
\hline & $(\mu \mathbf{m})$ & (ms) & & $(\mathbf{m s})$ & & $\left(10^{-20} \mathrm{~cm}^{2}\right)$ & & $(\mathrm{ms})$ & & $\left(10^{-20} \mathrm{~cm}^{2}\right)$ \\
\hline $\mathrm{W} \rightarrow \mathrm{X}$ & 5.5 & \multirow{3}{*}{0.01} & 0.007 & \multirow{3}{*}{0.26} & \multirow{3}{*}{0.04} & $\mathrm{n} / \mathrm{a}$ & 0.003 & \multirow{3}{*}{0.36} & \multirow{3}{*}{0.03} & $\mathrm{n} / \mathrm{a}$ \\
\hline $\mathrm{W} \rightarrow \mathrm{Y}$ & 2.4 & & 0.069 & & & 1.05 & 0.133 & & & 1.46 \\
\hline $\mathrm{W} \rightarrow \mathrm{Z}$ & 1.3 & & 0.924 & & & 2.72 & 0.864 & & & 1.84 \\
\hline$X \rightarrow Y$ & 4.3 & \multirow{2}{*}{1.05} & 0.144 & \multirow{2}{*}{1.97} & \multirow{2}{*}{0.53} & 1.48 & 0.090 & \multirow{2}{*}{1.58} & \multirow{2}{*}{0.66} & 1.15 \\
\hline$X \rightarrow Z$ & 1.7 & & 0.856 & & & 0.70 & 0.910 & & & 0.93 \\
\hline$Y \rightarrow Z$ & 2.8 & 3.29 & 1.000 & 4.38 & 0.75 & 1.34 & 1.000 & 3.29 & 1.00 & 1.78 \\
\hline
\end{tabular}

\subsection{Conclusions}

$\mathrm{CaGa}_{2} \mathrm{~S}_{4}$ is a non-hygroscopic material capable of transmitting light to a wavelength of $\sim 10 \mu \mathrm{m}$. It can efficiently support radiative emission to $\sim 4 \mu \mathrm{m}$. Large $(1.5 \mathrm{~cm}$ length, $0.5 \mathrm{~mm}$ diameter) single crystals doped with $\mathrm{Dy}^{3+}$ have been grown by Pete Schunemann at Sanders. Two (of three) indices of refraction have been measured (near $1.0 \mu \mathrm{m}$ ) to be 2.40 and 2.25 , giving a $\Delta n$ of $0.15 . \mathrm{CaGa}_{2} \mathrm{~S}_{4}$ is a good candidate for mid-IR laser and polarizer applications. Also of particular promise in these areas is $\mathrm{CaGa}_{2} \mathrm{Se}_{4}$. This host material transmits out to $13 \mu \mathrm{m}$ and can efficiently support radiative emission to $\sim 7 \mu \mathrm{m}$. Future efforts could explore the ability to grow large single crystals of high optical quality, and to the extent it will support mid-IR lasing. GeGaAsS glass proved to be an ineffective host for $4.3 \mu \mathrm{m}$ lasing due to impurity absorptions in the host. 


\section{$7 \mathrm{CaGa}_{2} \mathrm{~S}_{4}$ Laser Results}

Figure 7.1 shows the laser/pumping schemes employed for $\mathrm{CaGa}_{2} \mathrm{~S}_{4}$. The goal of this work was to establish the credibility of $\mathrm{CaGa}_{2} \mathrm{~S}_{4}$ as a mid-IR laser host by lasing the 4.3$\mu \mathrm{m} X \rightarrow \mathrm{Y}$ transition, but since the pump sources and optics were available to us, the 2.4and $1.4-\mu \mathrm{m}$ transitions were also of interest since they could provide additional information to credit or discredit $\mathrm{CaGa}_{2} \mathrm{~S}_{4}$ as a laser host. The 1.4- $\mu \mathrm{m}$ transition is also of interest for telecommunications amplifiers, since this falls near the dispersion minimum in silica fibers. ${ }^{75}$

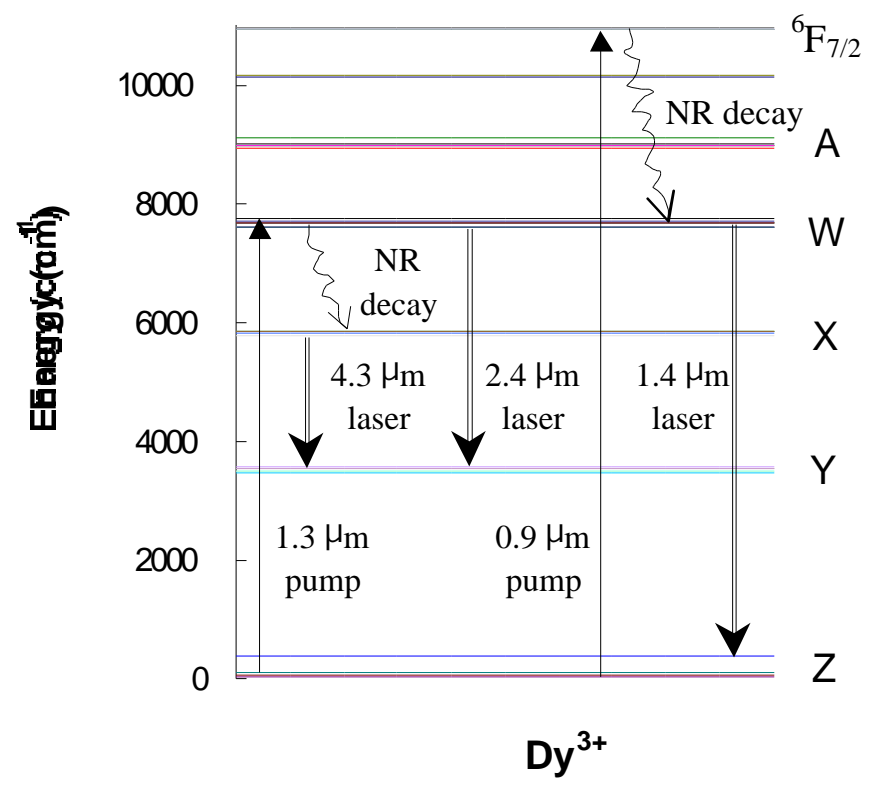

Figure 7.1. Dy ${ }^{3+}$ energy levels and lasing schemes for $\mathrm{CaGa}_{2} \mathrm{~S}_{4}: \mathrm{Dy}^{3+}$.

\section{$7.1 \mathrm{Dy}^{3+}$ at $4.3 \mu \mathrm{m}$}

The 4.3- $\mu$ m emission arises from the ${ }^{6} \mathrm{H}_{11 / 2} \rightarrow{ }^{6} \mathrm{H}_{13 / 2}$ transition $(X \rightarrow Y)$. A pulsed pump source at $1.3 \mu \mathrm{m}$ was available for direct pumping of the W-level. Non-radiative decay 
then populates the upper laser level (X). As the lower level lifetime is much longer than the upper level lifetime, population inversion can not be maintained at room temperature and CW operation is not feasible on this transition. A confocal laser cavity geometry was chosen as this is the least sensitive to cavity misalignment, while still providing a small beam waist.

\subsubsection{Predictions}

The laser waist is $370 \mu \mathrm{m}$ based on the geometry of the cavity, $w_{0}=\sqrt{\lambda L / 2 \pi}$, where $L$ is the resonator length. The pump spot profile was measured by a razor-scan and fit to a Gaussian (1/ $\mathrm{e}^{2}$ intensity) radial spot size of $354 \pm 10 \mu \mathrm{m}$ (See Appendix G). The cross section is given in Table 6.2 as $\sigma^{e m}=1.6 \times 10^{-20} \mathrm{~cm} 2$ at $4.3 \mu \mathrm{m}$. Since the exact Stark levels participating in the laser transition are not known, the Boltzmann occupation factors of these levels were estimated as $f_{2}=0.1$ and $f_{1}=0.1$ using (2.23) and the data for $\mathrm{Dy}^{3+}:$ YLF depicted in Figure 6.8. The pumping efficiency $\eta_{p, s}$ can be approximated by (2.80). The mode overlap efficiency $\eta_{\text {mode }}$ can be estimated from Figure 2.11 (page 2.6-61) for a waist ratio $a \approx 1.0$ and $r \approx 5$. Table 7.1 summarizes the predictions for absorbed threshold and slope for the $\mathrm{CaGa}_{2} \mathrm{~S}_{4}: \mathrm{Dy}^{3+} 4.3-\mu \mathrm{m}$ laser. Simulated laser output based on the computer model of Section 2.5 is shown in Figure 7.2 for two values of total output coupling $(1.7 \%$ and $8.5 \%)$. This simulation suggests $E_{t h}=3.3 \mathrm{~mJ}$ and $\eta_{\text {slope }}=$ $12.4 \%$ for the $8.5 \%$ output coupling. The model also produced a pumping efficiency of $\eta_{p}=63 \%$ (where $\left(1-\eta_{p}\right)$ equals the fraction of pump excitation lost to spontaneous decay during the pump pulse). 
Table 7.1. Values used for the threshold and slope estimates for the $\mathrm{CaGa}_{2} \mathrm{~S}_{4}: \mathrm{Dy}^{3+}$ laser at $4.3 \mu \mathrm{m}$ according to (2.81) and (2.88).

\begin{tabular}{|c|c|c|c|}
\hline$\lambda_{l}$ & 4.3 & $\mu \mathrm{m}$ & laser wavelength \\
\hline$\lambda_{p}$ & 1.3 & $\mu \mathrm{m}$ & pump wavelength \\
\hline$\alpha_{p}$ & 0.4 & $\mathrm{~cm}^{-1}$ & absorption coefficient (at $\left.\lambda_{p}\right)$ \\
\hline$w_{l}$ & 370 & $\mu \mathrm{m}$ & laser spot size \\
\hline$w_{p}$ & 354 & $\mu \mathrm{m}$ & pump spot size \\
\hline$\sigma^{e m}$ & 1.6 & $10^{-20} \mathrm{~cm}^{2}$ & emission cross section (at $\left.\lambda_{l}\right)$ \\
\hline$L$ & 1.0 & $\%$ & round-trip passive losses \\
\hline$T$ & 8.5 & $\%$ & total transmission \\
\hline$\eta_{p, s}$ & 95 & $\%$ & $\begin{array}{c}\text { pumping efficiency for } \\
\text { equivalent square pulse }\end{array}$ \\
\hline$f_{2} /\left(f_{l}+f_{2}\right)$ & 50 & $\%$ & bottlenecking factor \\
\hline$\eta_{\text {mode }}$ & 93 & $\%$ & mode overlap efficiency \\
\hline \hline $\boldsymbol{E}_{\text {th }}$ & $\mathbf{2 . 0}$ & $\mathbf{m J}$ & absorbed energy threshold \\
\hline$\eta_{\text {slope }}$ & $\mathbf{1 1 . 9}$ & $\mathbf{\%}$ & slope efficiency \\
\hline
\end{tabular}

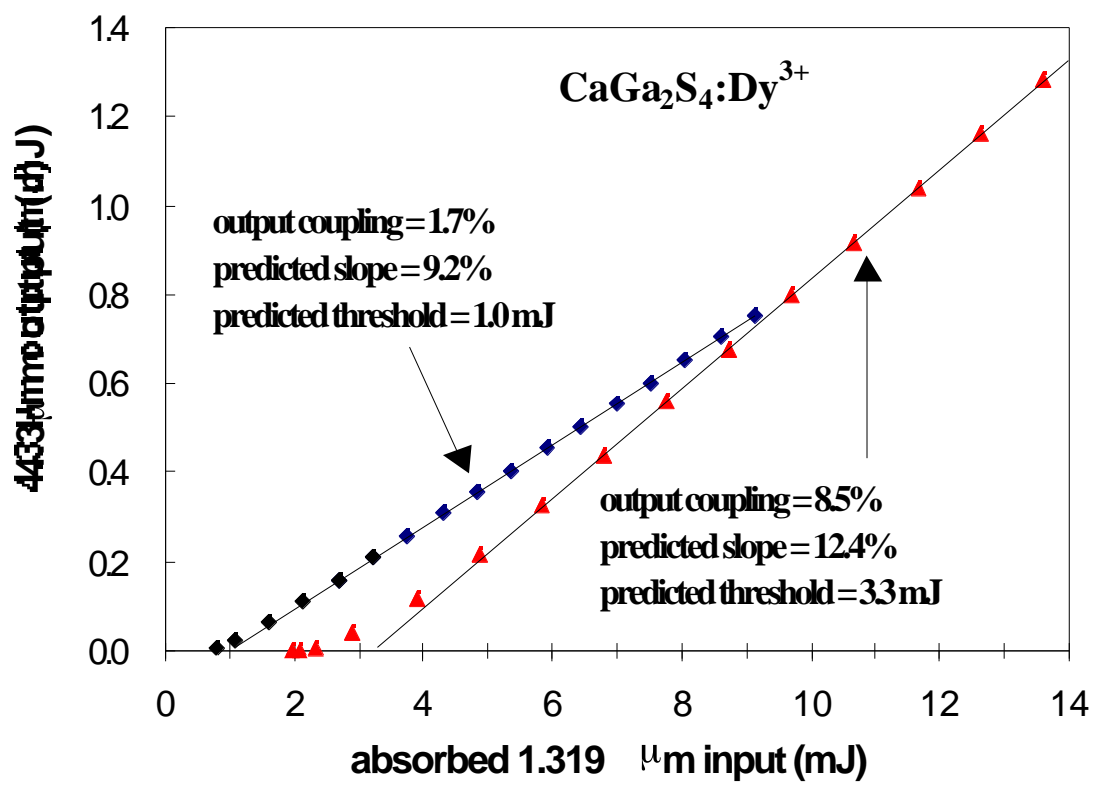

Figure 7.2. Predicted thresholds and slope efficiencies for $4.3 \mu \mathrm{m}$ laser action in $\mathrm{CaGa}_{2} \mathrm{~S}_{4}: \mathrm{Dy}^{3+}$. 


\subsubsection{Experimental Set-up}

The uncoated $1.5 \mathrm{~cm} \mathrm{CaGa} \mathrm{S}_{4}: \mathrm{Dy}^{3+}$ sample $\left(2 \mathrm{~mol} . \% \mathrm{Dy}^{3+}\right.$ in the melt) was placed in the center of a $20 \mathrm{~cm}$ confocal cavity and end-pumped at normal incidence by a $\mathrm{Nd}$ :YAG laser operating at $1.319 \mu \mathrm{m}$ (and $1.338 \mu \mathrm{m}$ ) with $75 \mu \mathrm{s}, 1 \mathrm{~Hz}$ pulses. To avoid Poynting vector walk-off along the two polarization axes of the sample and maximize absorption, the input beam was polarized along the 'fast' axis with a calcite polarizer. Poynting vector walk-off could be observed with a He-Ne laser $(632 \mathrm{~nm})$ polarized at $\sim 45^{\circ}$ with respect to the 'fast' and 'slow' axis. A beam separation of $\sim 1 \mathrm{~mm}$ over the $1.5 \mathrm{~cm}$ length of sample was observed. The pump spot profile was measured by a razor scan and fit to a Gaussian (1/ $\mathrm{e}^{2}$ intensity) radial spot size of $354 \pm 10 \mu \mathrm{m}$. A $1500 \mathrm{~nm}$ long-pass filter and $10 \mathrm{~cm} \mathrm{CaF}_{2}$ lens imaged the IR laser light at a $77 \mathrm{~K} \mathrm{InSb}$ detector for temporal waveform acquisition, or to a Molectron J3-09 pyroelectric detector for slope-efficiency measurements. A glass slide pick-off and Molectron J-25 pyroelectric detector measured the input pump energy. The laser output spectra were recorded with a 1-meter, 150 groove $/ \mathrm{mm}$ grating monochromator located $\sim 2$ meters from the cavity. Although the sample had a 'laser-grade' polish, its end faces exhibited a wedge angle of $\sim 3 \mathrm{mrad}$ as determined with a He-Ne laser. The set-up is similar to that shown in Figure 5.5 for $\mathrm{KPb}_{2} \mathrm{Cl}_{5}: \mathrm{Dy}^{3+}$. A picture of the author with the $\mathrm{CaGa}_{2} \mathrm{~S}_{4}: \mathrm{Dy}^{3+}$ laser cavity is shown in Figure 7.3. 


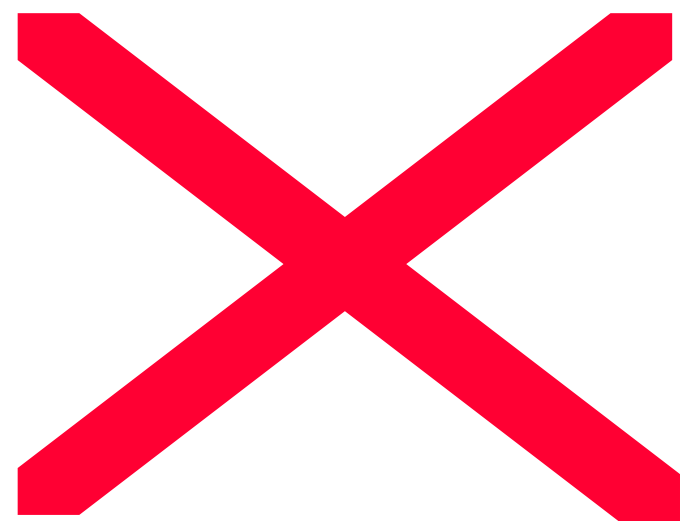

Figure 7.3. The author is pictured here with the $\mathrm{CaGa}_{2} \mathrm{~S}_{4}: \mathrm{Dy}^{3+}$ laser cavity in the foreground and the Cobra 1725 pump laser in the background. The laser crystal is colored green due to a He-Ne alignment laser.

\subsubsection{Laser Data and Analysis}

Four cavity mirrors were employed for $4.3 \mu \mathrm{m}$ operation with transmissions of $0.84 \%$ (high reflector or 'HR'), $0.84 \%, 2.55 \%$, and $7.65 \%$ (output coupler or 'OC'). Data was recorded for three mirror configurations with total transmissions $(\mathrm{HR}+\mathrm{OC})$ of $1.68 \%$, $3.39 \%$, and $8.49 \%$. Pump energy was limited to avoid damage, whose threshold was estimated at $\sim 15 \mathrm{~J} / \mathrm{cm}^{2}$ of absorbed fluence.

Slope efficiency curves are shown in Figure 7.4 for two of the cavity configurations, and all data are summarized in Table 7.2, which includes the predicted 
values. A Findlay-Clay plot of absorbed energy threshold vs. total transmission (shown in Figure 7.5) yielded a passive loss of $\mathrm{L}=0.9 \% \pm 0.3 \%$. This low value is perhaps expected if the scattering losses obey a Rayleigh $\lambda^{-4}$ dependence and if the Fresnel losses imposed by the $\sim 3$ mrad sample wedge are compensated by the confocal cavity geometry.

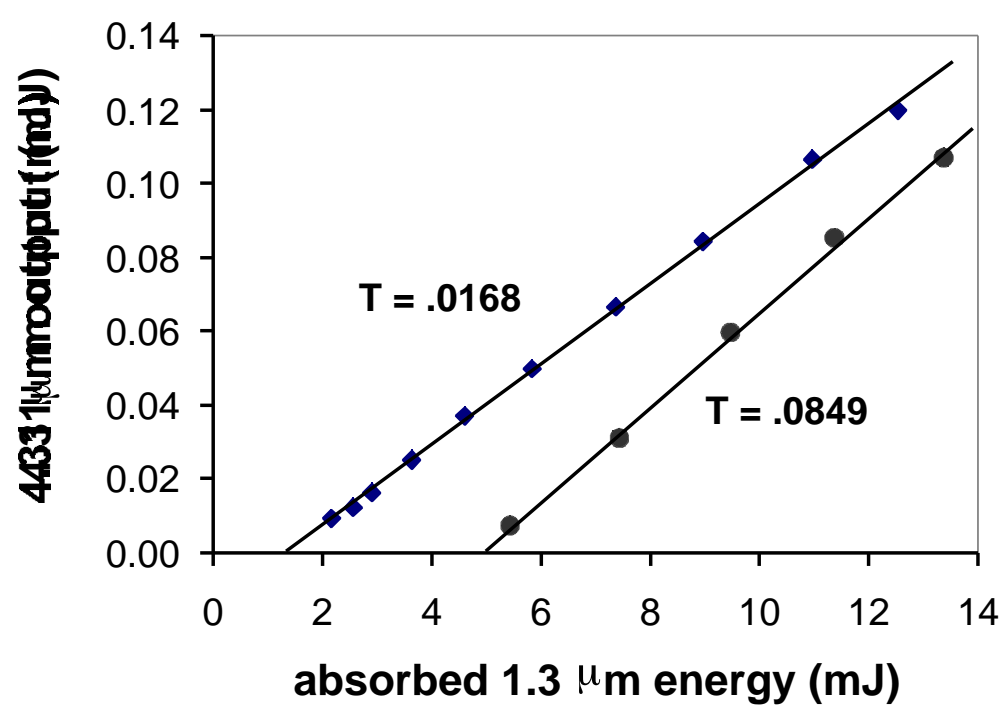

Figure 7.4. Slope-efficiency curves for $\mathrm{CaGa}_{2} \mathrm{~S}_{4}: \mathrm{Dy}^{3+}$ at $4.3 \mu \mathrm{m}$. T is the total mirror transmission (HR + OC).

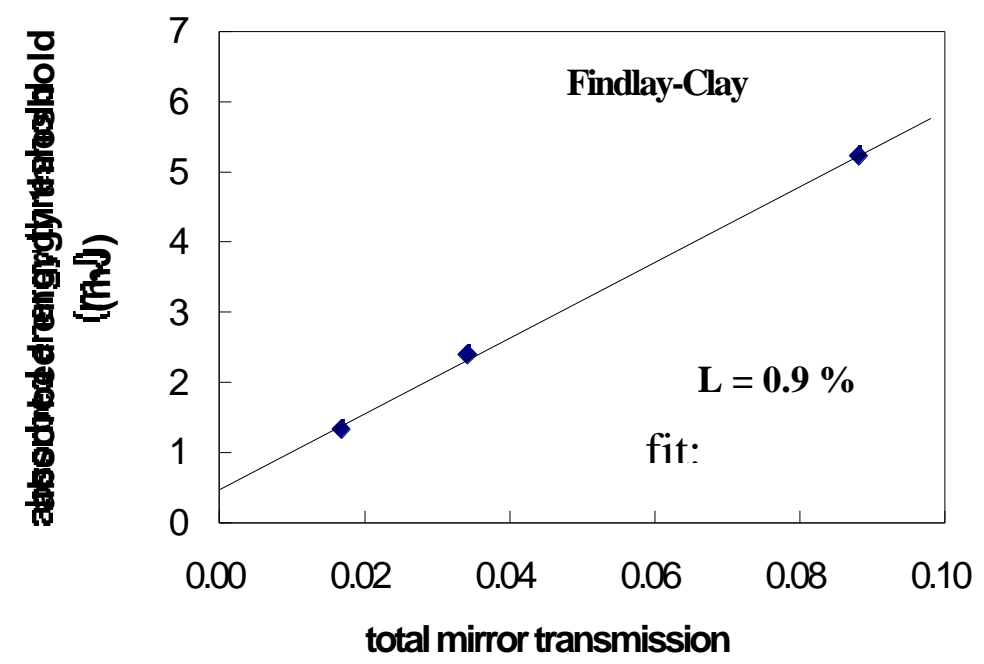

Figure 7.5. Findlay-Clay analysis of the $\mathrm{CaGa}_{2} \mathrm{~S}_{4}: \mathrm{Dy}^{3+} 4.3-\mu \mathrm{m}$ laser according to Section 2.6. The data imply a round-trip passive loss of $L=$ $0.9 \% \pm 0.3 \%$. 
Observed and calculated thresholds agreed to within experimental errors, but observed slope efficiencies were an order of magnitude low. The discrepancy has not been resolved. AR-coated samples, and samples with better optical quality, may perform better. Notice that the slope efficiencies do not vary greatly with the output coupling, as expected when the passive losses $\mathrm{L} \ll \mathrm{T}$.

Table 7.2. Summary of $\mathrm{CaGa}_{2} \mathrm{~S}_{4}: \mathrm{Dy}^{3+} 4.31 \mu \mathrm{m}$ laser threshold and slope data.

\begin{tabular}{|c|c|c|c|c|}
\hline $\begin{array}{c}\text { Total output } \\
\text { coupling } \\
(\boldsymbol{\%})\end{array}$ & $\begin{array}{c}\text { Measured } \\
\text { threshold } \\
(\mathbf{m J})\end{array}$ & $\begin{array}{c}\text { Calculated } \\
\text { threshold } \\
(\mathbf{m J})\end{array}$ & $\begin{array}{c}\text { Measured } \\
\text { slope } \\
\text { efficiency } \\
(\boldsymbol{\%})\end{array}$ & $\begin{array}{c}\text { Calculated } \\
\text { slope } \\
\text { efficiency } \\
(\boldsymbol{\%})\end{array}$ \\
\hline 1.7 & 1.3 & 1.0 & 1.1 & 9.2 \\
\hline 3.4 & 2.4 & 1.5 & 1.6 & 11.0 \\
\hline 8.5 & 5.2 & 3.3 & 1.4 & 12.4 \\
\hline
\end{tabular}

The laser output and fluorescence emission spectra are shown in Figure 7.6. Due to strong fundamental $\mathrm{CO}_{2}$ absorption, the cavity was purged with dry nitrogen for operation near $4.3 \mu \mathrm{m}$. The $\mathrm{CO}_{2}$ absorption coefficient was measured in our lab to be $0.0025 \mathrm{~cm}^{-1}$ at $4.3 \mu \mathrm{m}$, see Figure 7.6. This measurement was accomplished by comparing the emission spectra of a Blackbody source of known temperature when operated in a purged and unpurged ( 4 meter) path. The laser output of an unpurged cavity (peak wavelength $4.38 \mu \mathrm{m}$ ) is also shown in this figure for comparison. The wavelength-dependent loss imposed by the $\mathrm{CO}_{2}$ absorption $\left(2 \alpha_{\mathrm{CO}_{2}} \mathrm{~L} \sim 10 \%\right.$ per pass $)$ shifted lasing to a longer wavelength. These data suggest tuning is possible in the range $4.25-4.70 \mu \mathrm{m}$. The purged-cavity laser spectrum near $4.31 \mu \mathrm{m}$ has been filtered by the $\mathrm{CO}_{2}$ absorption along the $\sim 2$ meters of unpurged pathlength between the laser and the monochromator. The laser light was polarized along the 'fast' sample axis. 


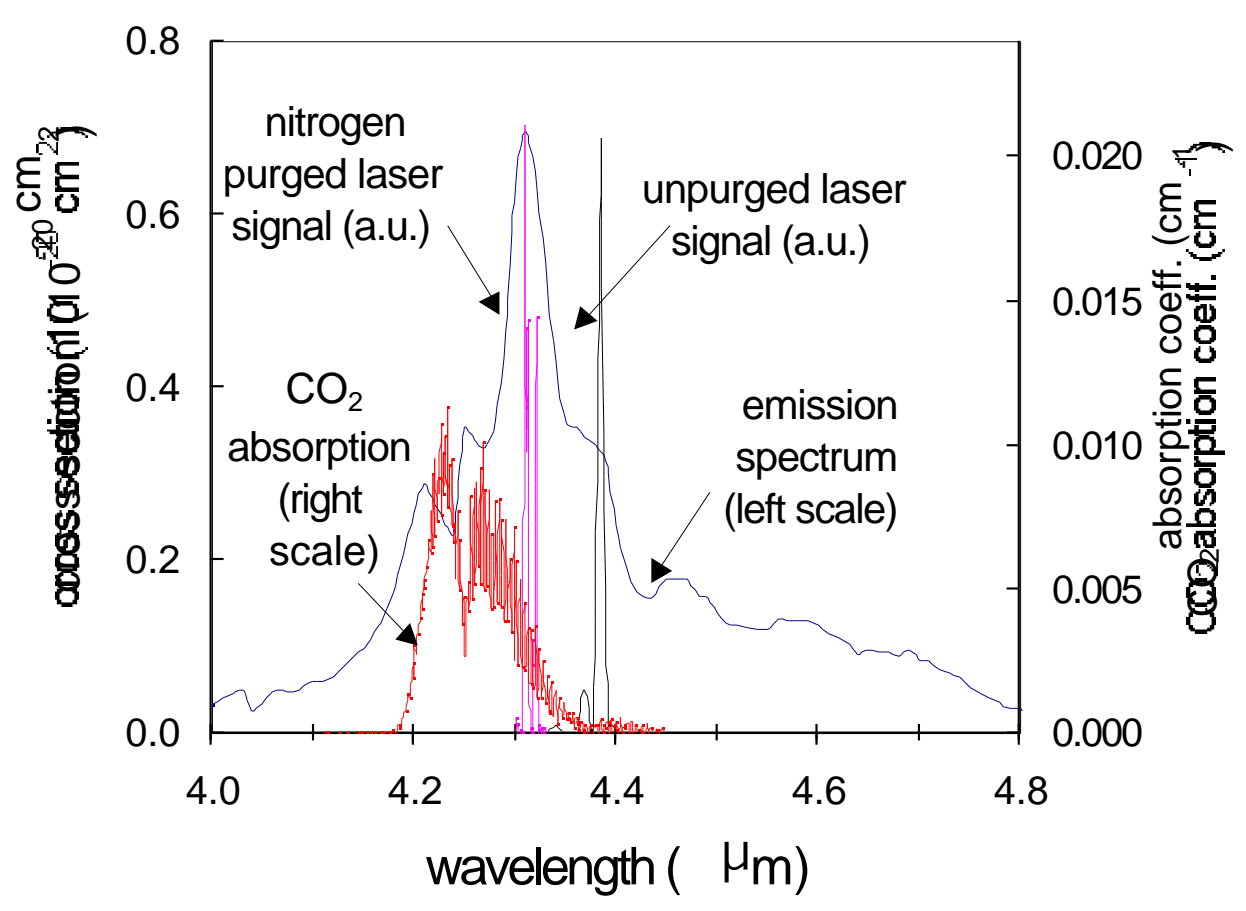

Figure 7.6. Emission spectrum and laser output near $4.3 \mu \mathrm{m}$ in $\mathrm{CaGa}_{2} \mathrm{~S}_{4}: \mathrm{Dy}^{3+}$. When purged with nitrogen, the laser operates at the peak $(4.31 \mu \mathrm{m})$ of the fluorescence spectrum. When unpurged, the added loss due to ambient $\mathrm{CO}_{2}$ absorption shifts the laser to a longer wavelength $(4.38 \mu \mathrm{m})$.

\section{$7.2 \mathrm{Dy}^{3+}$ at $2.4 \mu \mathrm{m}$}

$\mathrm{CaGa}_{2} \mathrm{~S}_{4}: \mathrm{Dy}^{3+}$ also lased on the $2.4-\mu \mathrm{m} \mathrm{W} \rightarrow \mathrm{Y}$ transition. The $\mathrm{W}$-level was pumped at 1.3 $\mu \mathrm{m}$ in the same cavity configuration as the $4.3 \mu \mathrm{m}$ laser of the previous section.

\subsubsection{Predictions}

The laser waist, calculated from the cavity geometry, $w_{0}=\sqrt{\lambda L / 2 \pi}$, is $278 \mu \mathrm{m}$. The pump spot profile was measured by a razor-scan and fit to a Gaussian (1/ $\mathrm{e}^{2}$ intensity) radial spot size of $354 \pm 10 \mu \mathrm{m}$ (See Appendix G). The cross section is given in Table 6.2 as $\sigma^{e m}=1.2 \times 10^{-20} \mathrm{~cm}^{2}$. The Boltzmann occupation factors were estimated as $f_{2}=0.1$ and $f_{l}=0.1$ using (2.23) and the data for $\mathrm{Dy}^{3+}$ :YLF depicted in Figure 6.8. The mode 
overlap efficiency $\eta_{\text {mode }}$ can be estimated from Figure 2.11 (page 2.6-61) for a waist ratio $a \approx 1.3$ and $r \approx 5$. Table 7.3 summarizes the predictions for absorbed threshold and slope for the $\mathrm{CaGa}_{2} \mathrm{~S}_{4}: \mathrm{Dy}^{3+} 2.4-\mu \mathrm{m}$ laser.

Table 7.3. Values used for the threshold and slope estimates for the $\mathrm{CaGa}_{2} \mathrm{~S}_{4}: \mathrm{Dy}^{3+}$ laser at $2.4 \mu \mathrm{m}$ according to $(2.81)$ and (2.88).

\begin{tabular}{|c|c|c|c|}
\hline$\lambda_{l}$ & 2.4 & $\mu \mathrm{m}$ & laser wavelength \\
\hline$\lambda_{p}$ & 1.3 & $\mu \mathrm{m}$ & pump wavelength \\
\hline$w_{l}$ & 278 & $\mu \mathrm{m}$ & laser spot size \\
\hline$w_{p}$ & 354 & $\mu \mathrm{m}$ & pump spot size \\
\hline$\sigma^{e m}$ & 1.2 & $10^{-20} \mathrm{~cm}^{2}$ & emission cross section $\left(\right.$ at $\left.\lambda_{l}\right)$ \\
\hline$L$ & 3.9 & $\%$ & round-trip passive losses \\
\hline$T$ & 9.4 & $\%$ & total transmission \\
\hline$\eta_{p}$ & 70 & $\%$ & $\begin{array}{c}\text { pumping efficiency for } \\
\text { equivalent square pulse }\end{array}$ \\
\hline$f_{2} /\left(f_{l}+f_{2}\right)$ & 50 & $\%$ & bottlenecking factor \\
\hline$\eta_{\text {mode }}$ & 85 & $\%$ & mode overlap efficiency \\
\hline \hline $\boldsymbol{E}_{\text {th }}$ & $\mathbf{3 . 8}$ & $\mathbf{m J}$ & absorbed energy threshold \\
\hline$\eta_{\text {slope }}$ & $\mathbf{1 1 . 4}$ & $\mathbf{\%}$ & slope efficiency \\
\hline
\end{tabular}

Simulated laser output based on the computer model of Section 2.5 is shown in Figure 7.7 for values of total output coupling $(0.9 \%$ and $9.4 \%)$. This simulation suggests $E_{t h}=$ $5.4 \mathrm{~mJ}$ and $\eta_{\text {slope }}=10.2 \%$ for the $9.4 \%$ output coupling. Note the difference in threshold is due to the fact that the Table 7.3 value predicts where laser output is greater than zero, whereas the simulation data uses the linear fit to the slope data, which is the method used in the actual measurement process. The model also produced a pumping efficiency of $\eta_{p}$ $=70 \%\left(\right.$ where $\left(1-\eta_{p}\right)$ equals the fraction of pump excitation lost to spontaneous decay during the pump pulse). 


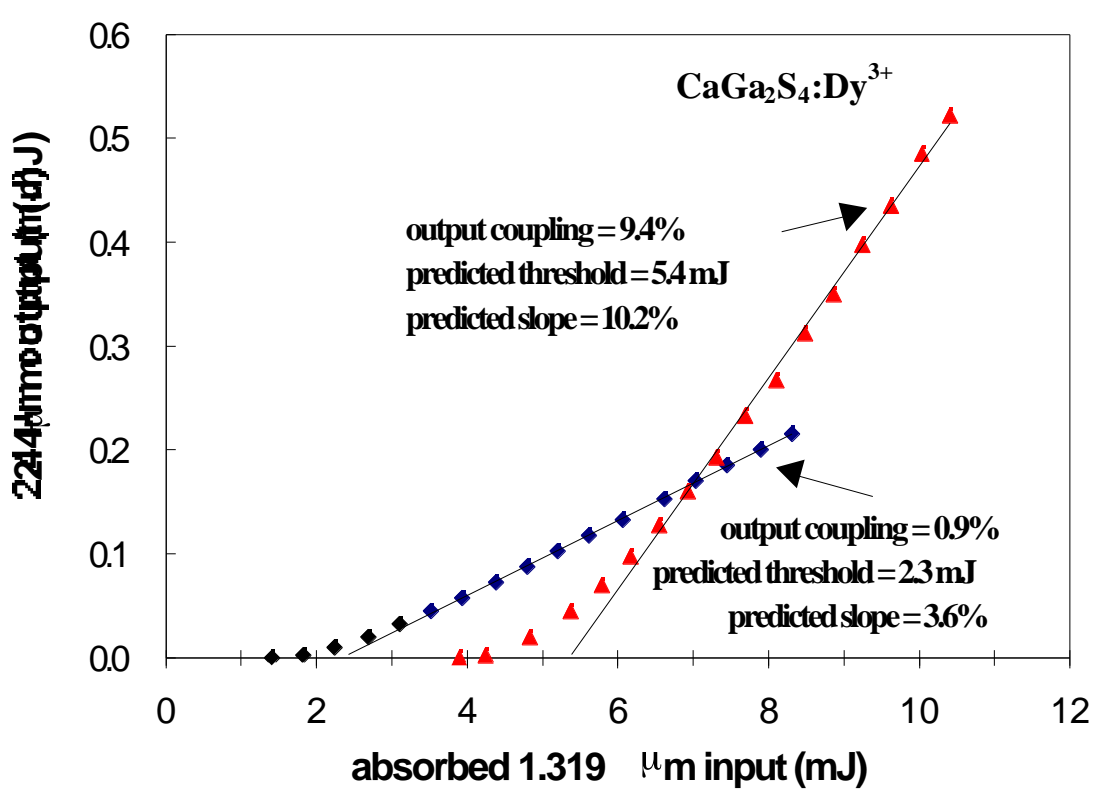

Figure 7.7. Predicted slope-efficiency curves for $\mathrm{CaGa}_{2} \mathrm{~S}_{4}: \mathrm{Dy}^{3+}$ at $2.4 \mu \mathrm{m}$.

\subsubsection{Experimental Set-up}

The cavity, pump, and detector apparatus used for the $4.3-\mu \mathrm{m}$ laser was used for the 2.4 $\mu \mathrm{m}$ laser, with the exception of the cavity mirrors.

\subsubsection{Laser Data and Analysis}

Four cavity mirrors were available for $2.4-\mu \mathrm{m}$ operation with transmissions of $0.36 \%$, $0.58 \%, 1.65 \%$, and $7.70 \%$. Data was recorded for four mirror configurations with total transmissions (high reflector + output coupler) of $0.94 \%, 2.23 \%, 8.28 \%$, and $9.35 \%$, where each configuration obeyed $\mathrm{T}_{\mathrm{HR}}<\mathrm{T}_{\mathrm{OC}}$. Slope efficiency curves are shown in Figure 7.8 for two of these configurations, and all data are summarized in Table 7.4. Absorbed energy was limited by concerns of damage to the sample. 


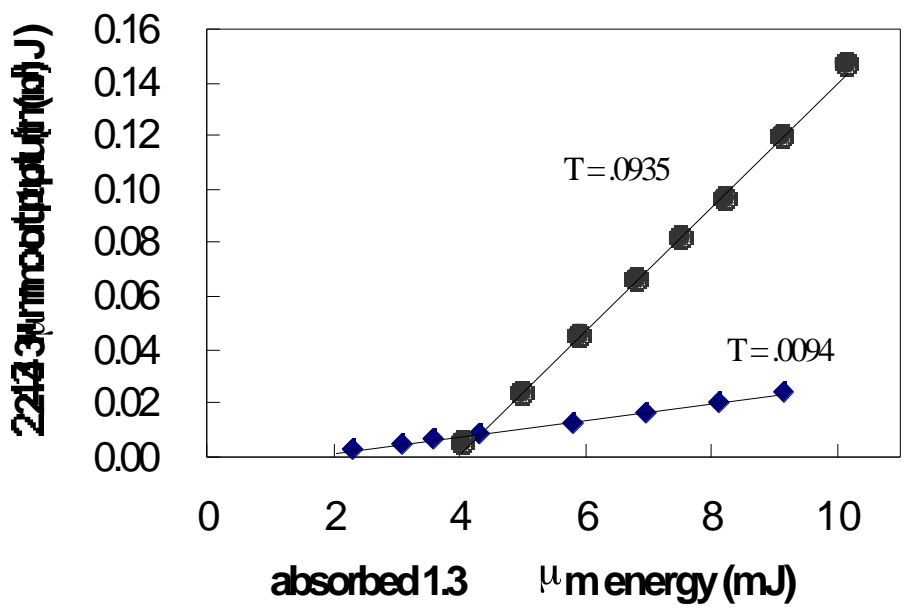

Figure 7.8. Slope-efficiency curves for $\mathrm{CaGa}_{2} \mathrm{~S}_{4}: \mathrm{Dy}^{3+}$ 2.4$\mu \mathrm{m}$ laser.

Table 7.4. Summary of $\mathrm{CaGa}_{2} \mathrm{~S}_{4}: \mathrm{Dy}^{3+}$ laser results.

\begin{tabular}{|c|c|c|c|c|}
\hline $\begin{array}{c}\text { Total } \\
\text { output } \\
\text { coupling } \\
(\mathbf{\%})\end{array}$ & $\begin{array}{c}\text { Measured } \\
\text { threshold } \\
(\mathbf{m J})\end{array}$ & $\begin{array}{c}\text { Predicted } \\
\text { threshold } \\
(\mathbf{m J})\end{array}$ & $\begin{array}{c}\text { Measured } \\
\text { slope } \\
\text { efficiency } \\
(\mathbf{\%})\end{array}$ & $\begin{array}{c}\text { Predicted } \\
\text { slope } \\
\text { efficiency } \\
(\mathbf{\%})\end{array}$ \\
\hline 0.9 & 1.3 & 2.3 & 0.3 & 3.6 \\
\hline 2.2 & 2.0 & 3.0 & 1.8 & 6.8 \\
\hline 8.3 & 3.6 & 4.9 & 2.7 & 9.9 \\
\hline 9.4 & 4.0 & 5.4 & 2.4 & 10.2 \\
\hline
\end{tabular}

A Findlay-Clay plot of absorbed energy threshold vs. transmission is shown in Figure 7.9 , yielding a round-trip passive loss of $\mathrm{L}=3.9 \% \pm 1.4 \%$. The laser output spectrum is shown in Figure 7.10, along with the unpolarized emission spectrum, showing that laser action occurs at the fluorescence peak. The laser light was determined to be polarized along the 'fast' axis (also the pump axis). 


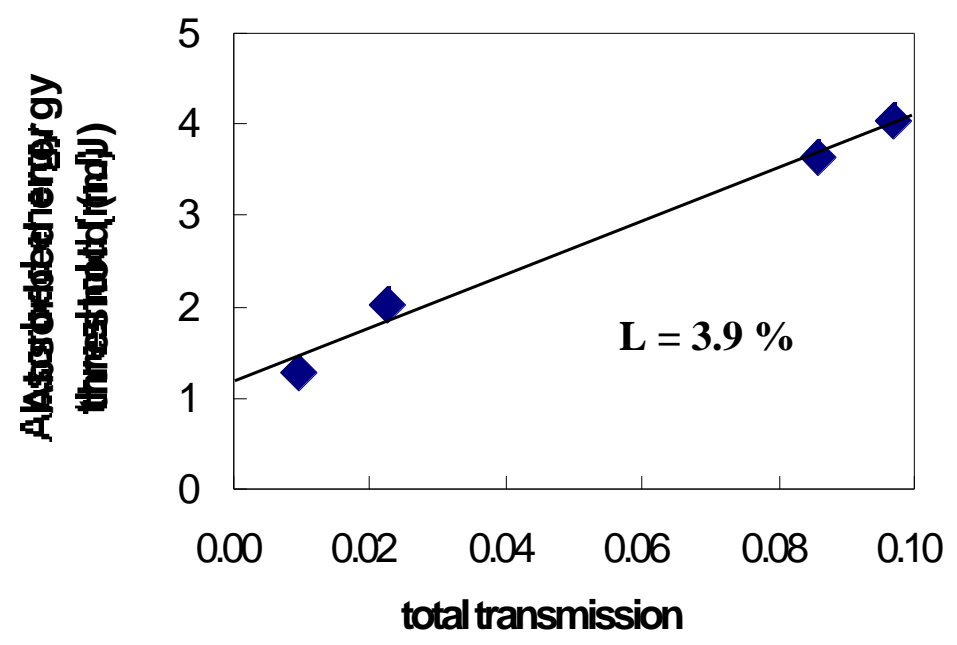

Figure 7.9. Findlay-Clay plot for the $\mathrm{CaGa}_{2} \mathrm{~S}_{4}: \mathrm{Dy}^{3+}$ laser at $2.4 \mu \mathrm{m}$. The data imply a passive loss of $3.9 \%$.

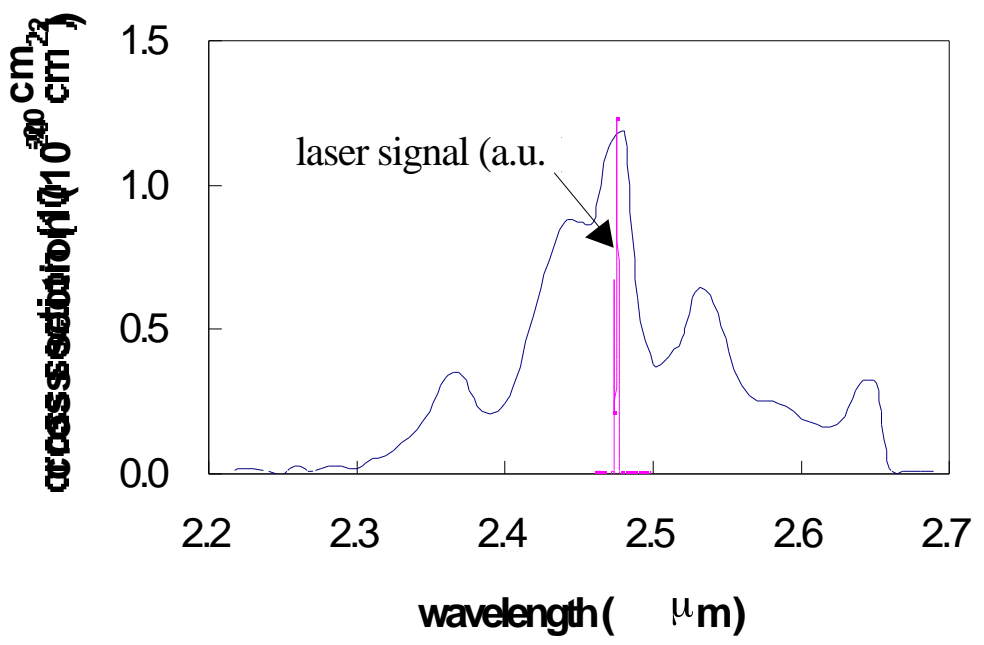

Figure 7.10. $\mathrm{CaGa}_{2} \mathrm{~S}_{4}: \mathrm{Dy}^{3+} 2.4-\mu \mathrm{m}$ emission showing laser signal and emission cross section.

$7.3 \mathrm{Dy}^{3+}$ at $1.4 \mu \mathrm{m}$

As mentioned previously, the small redshifted peaks in the absorption spectrum of $\mathrm{CaGa}_{2} \mathrm{~S}_{4}: \mathrm{Dy}^{3+}$ become large features in the emission spectrum. These correspond to emission to a Stark level which lies $\sim 400 \mathrm{~cm}^{-1}$ above the ground-state, where roomtemperature thermal occupation is low. Thus the re-absorption is low (with respect to the 
passive losses and the output coupling) and the laser transitions can be classified as quasifour level. Laser action was achieved at $1.4 \mu \mathrm{m}$ on such a transition in the $\mathrm{W} \rightarrow \mathrm{Z}$ band, pumped at $910 \mathrm{~nm}\left({ }^{6} \mathrm{~F}_{7 / 2}\right.$ level $)$ by a $\mathrm{Cr}^{3+}: \mathrm{LiSAF}$ laser.

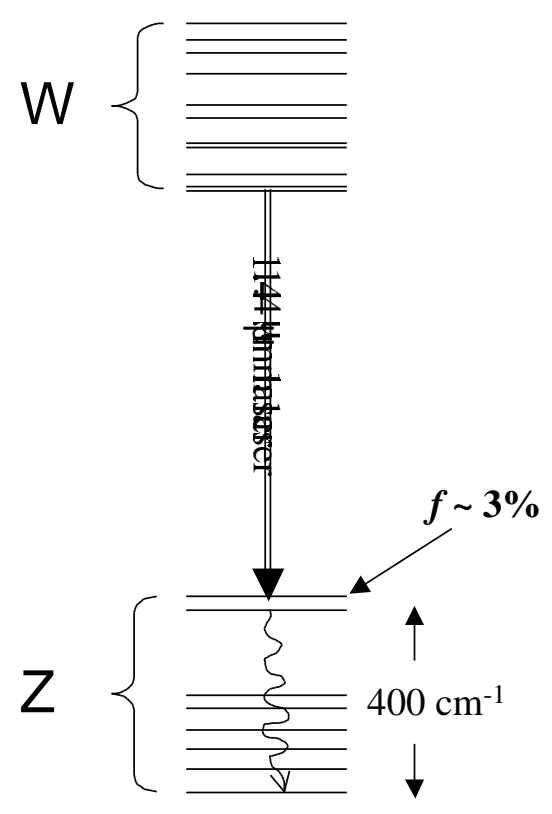

Figure 7.11. Stark-level structure (not to scale) of the $W$ and $Z$ (ground) manifolds of $\mathrm{CaGa}_{2} \mathrm{~S}_{4}: \mathrm{Dy}^{3+}$, showing the 1.4- $\mu \mathrm{m}$ laser transition. The exact Stark levels participating in the transition are not known.

\subsubsection{Predictions}

The pump spot profile was measured by a razor-scan and fit to a Gaussian ( $\left(1 / \mathrm{e}^{2}\right.$ intensity) radial spot size of $338 \pm 10 \mu \mathrm{m}$ (See Appendix G). The laser waist is $264 \mu \mathrm{m}$, calculated from the cavity geometry. The cross section is given in Table 6.2 as $\sigma^{e m}=1.4 \times 10^{-20}$ $\mathrm{cm}^{2}$. The Boltzmann occupation factors were estimated as $f_{2}=0.1$ and $f_{1}=0.03$. The mode overlap efficiency $\eta_{\text {mode }}$ can be estimated from Figure 2.11 (page 2.6-61) for a waist ratio $a \approx 1.3$ and $r \approx 5$. Table 7.5 summarizes the predictions for absorbed threshold and slope for the $\mathrm{CaGa}_{2} \mathrm{~S}_{4}: \mathrm{Dy}^{3+} 1.4-\mu \mathrm{m}$ laser. 
Table 7.5. Values used for the threshold and slope estimates for the $\mathrm{CaGa}_{2} \mathrm{~S}_{4}: \mathrm{Dy}^{3+}$ laser at $1.4 \mu \mathrm{m}$ according to (2.81) and (2.88).

\begin{tabular}{|c|c|c|c|}
\hline$\lambda_{l}$ & 1.4 & $\mu \mathrm{m}$ & laser wavelength \\
\hline$\lambda_{p}$ & 0.91 & $\mu \mathrm{m}$ & pump wavelength \\
\hline$w_{l}$ & 264 & $\mu \mathrm{m}$ & laser spot size \\
\hline$w_{p}$ & 338 & $\mu \mathrm{m}$ & pump spot size \\
\hline$\sigma^{e m}$ & 1.4 & $10^{-20} \mathrm{~cm}^{2}$ & emission cross section $\left(\right.$ at $\left.\lambda_{l}\right)$ \\
\hline$L^{*}$ & 13 & $\%$ & $\begin{array}{c}\text { round-trip passive and re- } \\
\text { absorption losses }\end{array}$ \\
\hline$T$ & 2.2 & $\%$ & total transmission \\
\hline$\eta_{p}$ & 55 & $\%$ & $\begin{array}{c}\text { pumping efficiency for } \\
\text { equivalent square pulse }\end{array}$ \\
\hline$f_{2} /\left(f_{l}+f_{2}\right)$ & 77 & $\%$ & bottlenecking factor \\
\hline$\eta_{\text {mode }}$ & 85 & $\%$ & mode overlap efficiency \\
\hline \hline $\boldsymbol{E}_{\text {th }}$ & $\mathbf{6 . 2}$ & $\mathbf{m J}$ & absorbed energy threshold \\
\hline$\eta_{\text {slope }}$ & $\mathbf{8 . 3}$ & $\%$ & slope efficiency \\
\hline
\end{tabular}

* includes $9 \%$ re-absorption losses

Simulated laser output based on the computer model of Section 2.5 is shown in Figure 7.12. This simulation suggests $E_{t h}=10.3 \mathrm{~mJ}$ and $\eta_{\text {slope }}=6.9 \%$. Note the difference in threshold is due to the fact that the Table 7.5 value predicts where laser output is greater than zero, whereas the simulation data uses the linear fit to the slope data, which is the method used in the actual measurement process. The model also produced a pumping efficiency of $\eta_{p}=55 \%$. 


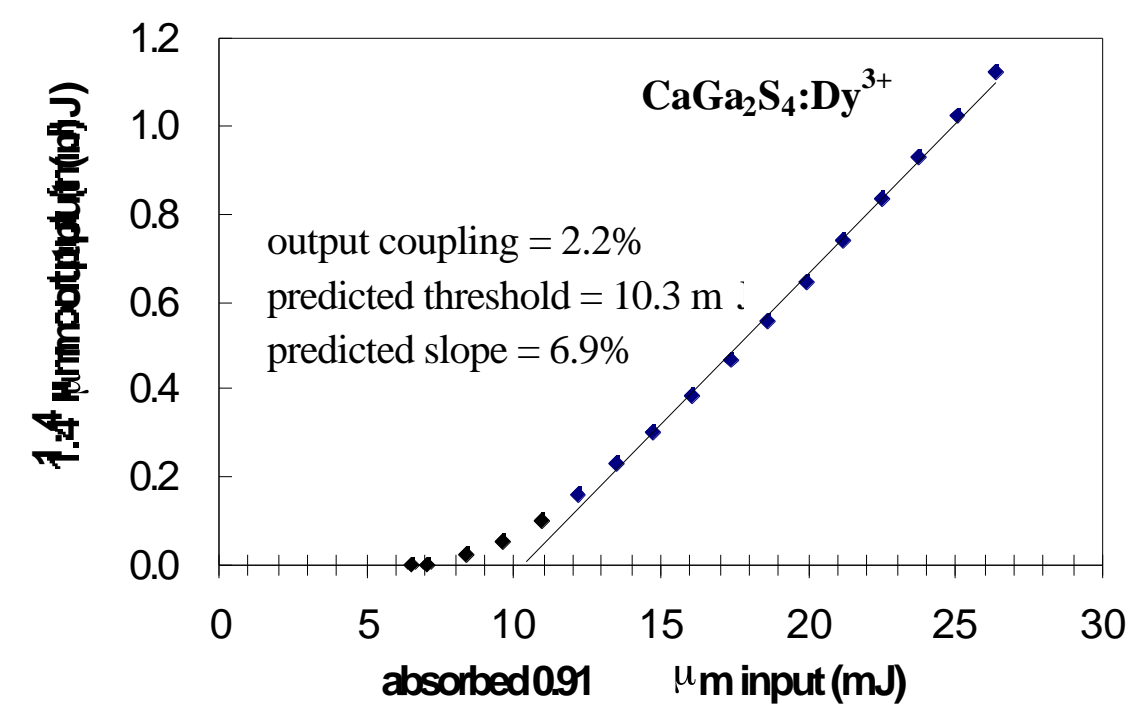

Figure 7.12. Predicted slope-efficiency curves for the $\mathrm{CaGa}_{2} \mathrm{~S}_{4}: \mathrm{Dy}^{3+}$ laser at $1.4 \mu \mathrm{m}$.

\subsubsection{Experimental Set-up}

The experimental set-up included a $20-\mathrm{cm}$ asymmetric resonator. The input mirror had a $1 \%$ transmission at the laser wavelength and a $30-\mathrm{cm}$ radius of curvature. The output mirror had a transmission of $1.2 \%$ at the laser wavelength and a radius of curvature of 40 $\mathrm{cm}$. The sample (same as that for 4.3- and 2.4- $\mu \mathrm{m}$ laser operation) was placed at the center of this cavity and end-pumped with $0.91-\mu \mathrm{m}$ light from a $\mathrm{Cr}^{3+}: \mathrm{LiSAF}$ laser operating at 1-Hz. The pump light was blocked with a $1-\mu \mathrm{m}$ long pass filter and the laser light was directed at a Molectron J3-09 pyroelectric detector for slope-efficiency measurements. A wedged glass pick-off and Molectron J-25 pyroelectric detector measured the input pump energy. Input power was varied by rotation of a circular variable attenuator. Laser emission was measured with an ANDO $^{\mathrm{TM}}$ optical spectrum analyzer (0.5 $\mathrm{nm}$ resolution). 


\subsubsection{Laser Data and Analysis}

The measured slope-efficiency curve for the 1.4- $\mu \mathrm{m}$ laser is shown in Figure 7.13. The slope-efficiency of this laser is still below the predicted value $(6.9 \%)$, but is relatively much better than the 4.3- and 2.4- $\mu$ m laser. In fact, the slope efficiency results seem to improve with the wavelength of the laser, suggesting some wavelength-dependent phenomenon is responsible for the low slope efficiencies. Perhaps impurities play a larger part as a loss mechanism at longer wavelengths.

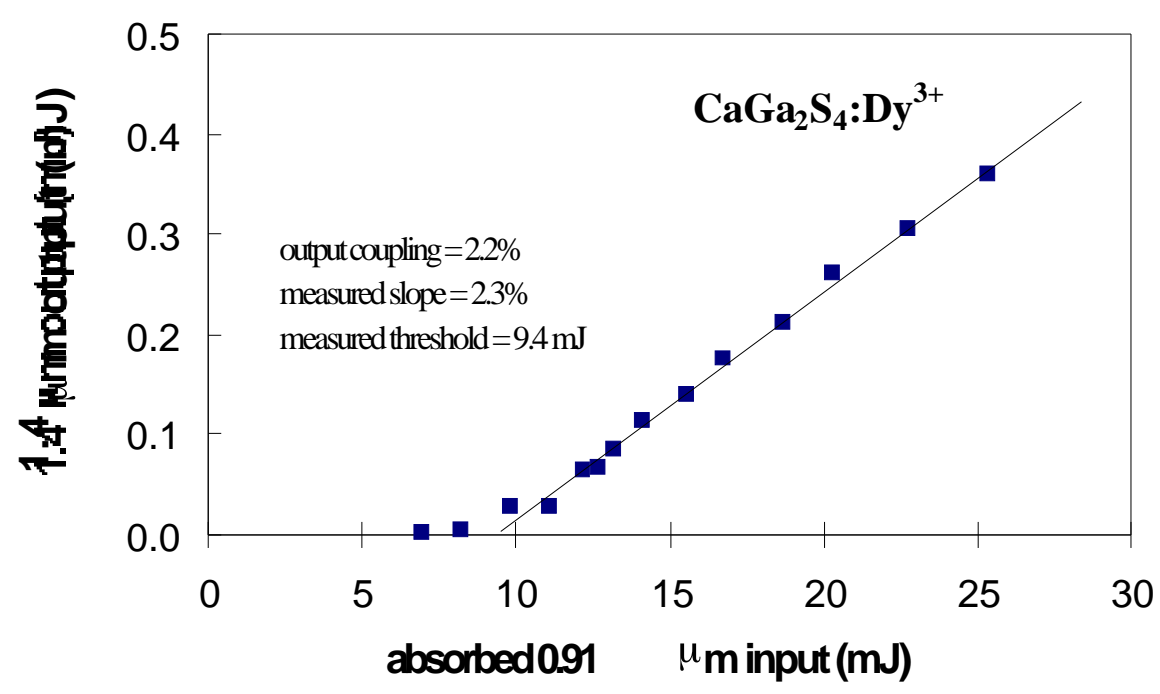

Figure 7.13. Measured slope-efficiency curve for the $\mathrm{CaGa}_{2} \mathrm{~S}_{4}: \mathrm{Dy}^{3+}$ laser at $1.4 \mu \mathrm{m}$. The total output coupling was $2.2 \%$.

The spectral content of the laser pulses is shown in Figure 7.14. Figure 7.15 shows that the emission occurs at the peak of the emission. Since this laser operates in a quasi-three level manner (i.e. there is non-negligible absorption at the laser wavelength), a McCumber-derived emission cross section has also been determined via (2.27) and (2.29), and is included in Figure 7.15. The results are in good agreement. The region beyond $1.4 \mu \mathrm{m}$ where the McCumber cross section is zero but the measured emission is 
greater than zero could be a result of the resolution of the measurement. The absorption measurement, upon which the McCumber cross section is based, had a resolution of a few $\mathrm{nm}$, while the measured cross section had a resolution of $>10 \mathrm{~nm}$.

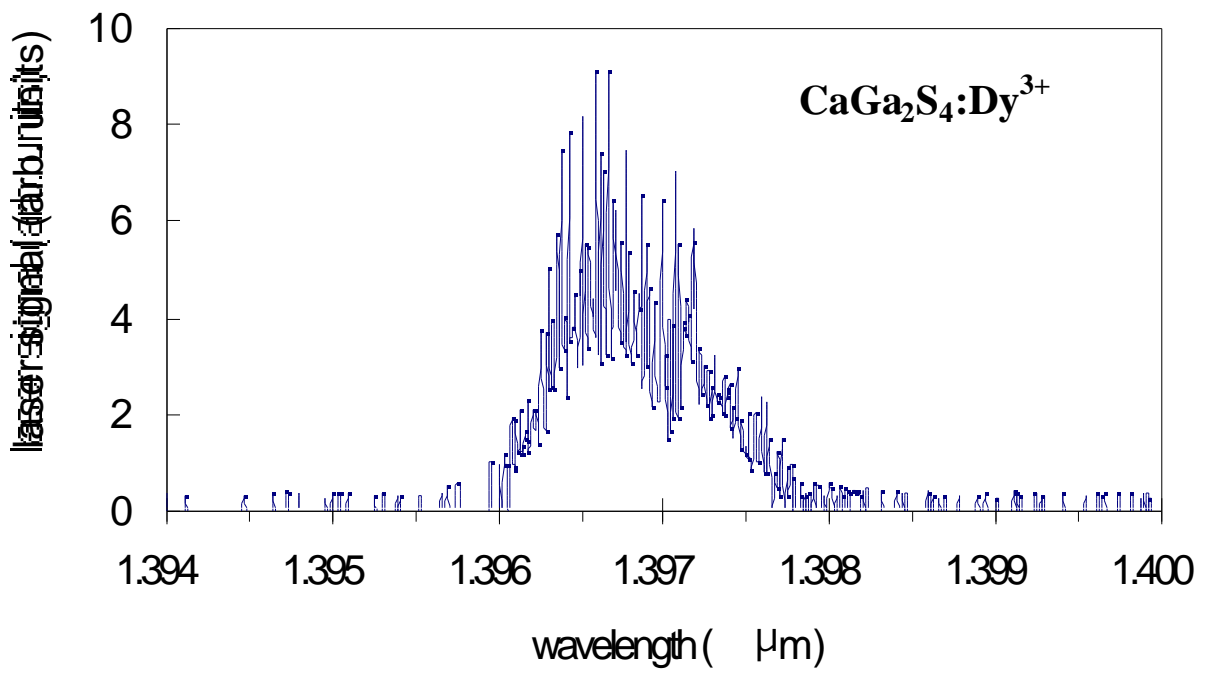

Figure 7.14. The laser emission is centered at $\sim 1.397 \mu \mathrm{m}$ with a FWHM of $\sim 1.5 \mathrm{~nm}$.

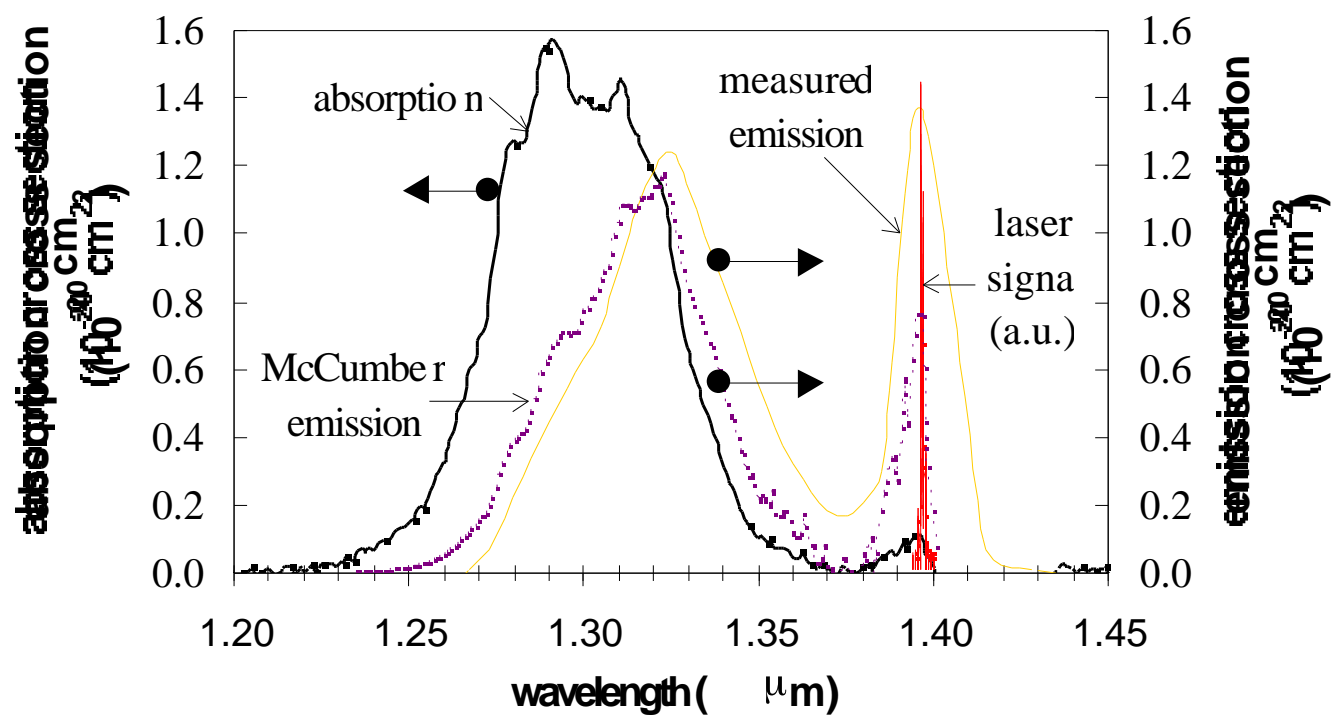

Figure 7.15. Absorption and emission cross section near $1.4 \mu \mathrm{m}$. The laser signal at $1.397 \mu \mathrm{m}$ is also shown for comparison. The McCumber-derived emission spectrum and the measured emission spectrum are in reasonably good agreement. 


\subsection{Conclusions}

$\mathrm{CaGa}_{2} \mathrm{~S}_{4}$ has proven to be a viable mid-IR host material, supporting lasing at 1.4-, 2.4-, and 4.3- $\mu \mathrm{m}$ in $\mathrm{CaGa}_{2} \mathrm{~S}_{4}: \mathrm{Dy}^{3+}$. These lasers represent the first room-temperature, rareearth-doped sulfide lasers to operate beyond $1.1 \mu \mathrm{m}$. 


\section{Summary}

In summary, the first rare-earth-doped solid-state sulfide and chloride lasers operating beyond $2 \mu \mathrm{m}$ in ambient conditions have been demonstrated. The key development was the identification of the host materials $\mathrm{CaGa}_{2} \mathrm{~S}_{4}$ and $\mathrm{KPb}_{2} \mathrm{Cl}_{5}$. These hosts are stable in ambient conditions, readily incorporate rare-earth ions, and have low maximum phonon energies. A maximum phonon energy of $200 \mathrm{~cm}^{-1}$ has been measured for $\mathrm{KPb}_{2} \mathrm{Cl}_{5}$ from Raman scattering data, and a maximum phonon energy of $\sim 350 \mathrm{~cm}^{-1}$ for $\mathrm{CaGa}_{2} \mathrm{~S}_{4}$ has been inferred from infrared cutoff measurements. Energy-Gap law ( $\left.\mathrm{W}^{\mathrm{MP}} \approx \operatorname{Bexp}[-\beta \Delta \mathrm{E}]\right)$ parameters have been determined for $\mathrm{KPb}_{2} \mathrm{Cl}_{5}$ to be $\mathrm{B}=3.72 \times 10^{9} \mathrm{~s}^{-1}$ and $\beta=1.16 \times 10^{-2}$ $\mathrm{cm}$, indicating that laser action should be possible to near $9 \mu \mathrm{m}$ in this host.

The $\mathrm{Dy}^{3+}$ ion was studied extensively in these crystalline hosts, as well as in $\mathrm{CaGa}_{2} \mathrm{Se}_{4}$ and the glassy host GeGaAsS. Radiative quantum efficiencies were determined using a novel technique based on emission spectra. A comparison of the radiative quantum efficiencies of these hosts is shown in Table 8.1.

Table 8.1. Radiative quantum efficiencies for $\mathrm{Dy}^{3+}$ energy levels in some of the hosts studied in this report.

\begin{tabular}{|c||c|c|c|}
\hline \multicolumn{1}{|c||}{} & \multicolumn{3}{c|}{ Dy $^{3+}$ Level } \\
\hline Host & A & W & X \\
\hline \hline $\mathrm{CaGa}_{2} \mathrm{Se}_{4}$ & 0.10 & 0.96 & 1.00 \\
\hline $\mathrm{KPb}_{2} \mathrm{Cl}_{5}$ & 0.01 & 0.79 & 1.00 \\
\hline $\mathrm{CaGa}_{2} \mathrm{~S}_{4}$ & 0.00 & 0.20 & 0.98 \\
\hline $\mathrm{GeGaAsS}^{1}$ & 0.00 & 0.03 & 0.66 \\
\hline
\end{tabular}


The results of this method were compared to those of a Judd-Ofelt analysis, the standard technique which is based on absorption data. While the results agreed in general, the emission-based proved to be a much more accurate method. The Judd-Ofelt method, however, remains a vastly important tool due to it's broad applicability. The emission technique can only address data that can be detected in an emission spectrum, whereas virtually all decay channels can be accessed by the Judd-Ofelt method. An important parameter for mid-IR transitions in the Judd-Ofelt method is the transition line strength for magnetic dipole transitions. As the literature is often concerned with near-IR/visible transitions, these values are not heavily reported. In this report, magnetic dipole line strengths are compiled for many rare-earths. These values are completely independent of host and therefore of very general use.

A cross-relaxation mechanism was discovered to be important factor in the decay of the $\mathrm{W}$-level in $\mathrm{KPb}_{2} \mathrm{Cl}_{5}: \mathrm{Dy}^{3+}$, and a cross-relaxation coefficient $k=1.83 \times 10^{-37} \mathrm{~cm}^{6} \mathrm{~s}^{-1}$ was measured, where $\mathrm{W}^{\mathrm{c}}=k \mathrm{~N}_{0}^{2}$. Here, $\mathrm{W}^{\mathrm{c}}$ in the cross-relaxation decay rate, and $\mathrm{N}_{0}$ is the total $\mathrm{Dy}^{3+}$ concentration. This value compared well with that estimated theoretically from the Burshtein 'hopping' model.

In order to understand and predict mid-IR laser performance, a numerical model using fourth-order Runge-Kutta techniques ${ }^{76}$ was developed an implemented in standard spreadsheet software. Coupled differential equations were solved to determine cavity photon number and population inversion inside the laser cavity. Predicted slopeefficiency curves were generated for each laser configuration.

Future work in the area of solid-state mid-IR lasers will undoubtedly reveal new hosts and longer wavelengths. It is hoped that the issues and techniques developed in this 
report will provide a solid foundation by which future researchers can further advance the field of mid-IR laser technology, perhaps attaining the benchmark of a $10 \mu \mathrm{m}$ rare-earthdoped, ambient-condition laser. 


\section{Appendices}

\section{Appendix A : Derivation of integrated cross section relation}

In this section we derive the relation (2.28):

$$
g_{u} \quad \sigma^{e m}(v) d v=g_{l} \quad \sigma^{a b s}(v) d v .
$$

Referring to Figure 2.3, we can write

$$
\sigma^{e m}(v)={ }_{j=1}^{g_{u}}{ }_{i=1}^{g_{l}} f_{j} \sigma_{j i}(v)
$$

and

$$
\sigma^{a b s}(\boldsymbol{v})={ }_{i=1}^{g_{l}}{ }_{j=1}^{g_{u}} f_{i} \sigma_{i j}(v)
$$

If we assume that the cross section are independent of $j$ and $i$, but are still frequency dependent, we can use reciprocity $\sigma_{j i}=\sigma_{i j} \equiv \bar{\sigma}$ to write

$$
\sigma_{j i}(v)=\bar{\sigma} g^{e m}(v) \quad \text { and } \quad \sigma_{i j}(v)=\bar{\sigma} g^{a b s}(v)
$$

where $g^{e m}(v)$ and $g^{a b s}(v)$ are the normalized emission and absorption lineshape functions, respectively. Performing the sums in (9.2) and (9.3) and using $\Sigma f=1$, we obtain

$$
\sigma^{e m}(v)=\bar{\sigma} g_{l} g^{e m}(v)
$$

and

$$
\sigma^{a b s}(v)=\bar{\sigma} g_{u} g^{a b s}(v)
$$

Finally, we can integrate (9.5) and (9.6) over frequency to obtain 


$$
\sigma^{e m}(v) d v=\bar{\sigma} g_{l}
$$

and

$$
\sigma^{a b s}(v) d v=\bar{\sigma} g_{u} .
$$

Upon diving these last two expressions, we obtain the desired result (2.28). 


\section{Appendix B : Local field correction in dielectrics}

When dealing with electric and magnetic fields in dielectrics, correction need to be made for local field effects. Normally, electric $\vec{E}$ and magnetic $\vec{B}$ fields refer to the macroscopic or average field inside the medium. At the location of an individual ion where absorption and emission processes occur, however, the local field $\vec{E}_{l o c}$ may be different from the average field because the radiation polarizes the atomic environment. Two corrections need to be considered for radiative transition probabilities, one for the local field $\left(\vec{E}_{l o c} / \vec{E}\right)$ and one for the inclusion of refractive index $n$ and dielectric constant $\varepsilon$.

The local field is generally not known for an arbitrary crystal site. The case of high local symmetry is known, however, and it is customary to use this correction factor for all cases. Other factors have been proposed and experimentally studied, ${ }^{77}$ but these will not be considered here. The local field in crystals of high local symmetry is given by $^{78}$

$$
\vec{E}_{l o c}=\vec{E}+\frac{4}{3} \pi \vec{P}
$$

where $\vec{P}$ is the polarization and is related to $\vec{E}$ by the linear susceptibility, $\vec{P}=\chi \vec{E}$. The dielectric constant can be expressed in terms of the susceptibility as $\varepsilon=1+4 \pi \chi$. Solving for $\vec{E}_{l o c} / \vec{E}$ we obtain

$$
\frac{\vec{E}_{l o c}}{\vec{E}}=\frac{\varepsilon+2}{3}
$$

The electric-dipole spontaneous transition probability is given by Fermi's Golden Rule as 


$$
A=\frac{2 \pi}{\hbar^{2}}\left\langle\left.\langle f|e \vec{r} ? \vec{E}| i\rangle\right|^{2} \rho(\omega),\right.
$$

where $\langle f|$ and $|i\rangle$ represent the final and initial states, respectively. The density of states $\rho(\omega)$ is proportional to $u(\omega) / \varepsilon$, where $u(\omega)$ is the energy density given by

$$
u(\omega)=\frac{\hbar \omega^{3} n^{3}}{\pi^{2} c^{3}} n_{\omega},
$$

where $n_{\omega}$ is the mode occupation number. Thus $A$ is proportional to $\left(n^{3} / \varepsilon\right)|\vec{E}|^{2}$, so that the total correction factor is

$$
\frac{n^{3}}{\varepsilon} \frac{\varepsilon+2}{3} \sqrt{\downarrow}
$$

For electric dipole transitions we take $\varepsilon=n^{2}$, and (9.13) becomes

$$
\chi_{A}^{E D}=n \frac{n^{2}+2}{3} \sqrt[V]{ }^{2} .
$$

For magnetic dipole transitions we can take $\varepsilon \rightarrow 1$ (non-magnetic materials), and (9.13) becomes

$$
\chi_{A}^{M D}=n^{3}
$$

We have seen in $(2.16)$ that the cross section $\sigma$ is related to the spontaneous transition rate $A$ by $\sigma \propto A / n^{2}$. It follows directly that

$$
\chi_{\sigma}^{E D}=\frac{1}{n} \frac{n^{2}+2}{3} y^{2} \quad \text { and } \quad \chi_{\sigma}^{E D}=n
$$




\section{$\underline{\text { Appendix } \mathrm{C}: \text { Calculations of } S^{M D} \text { for various rare-earth ions }}$}

The magnetic dipole line strengths $S^{M D}$ are calculated by (2.68) and (2.69). In practice this is done by calculating the matrix elements in the pure $L-S$ states and transforming to the intermediate-coupled states. The transformation matrix that accomplishes this is

$$
\tilde{M}^{\prime}=\tilde{E} \tilde{M} \tilde{E}^{-1}
$$

where $\tilde{M}^{\prime}$ are the reduced matrix elements in the intermediate scheme, $\tilde{E}$ is the matrix of energy eigenvectors of intermediate coupled states containing the coefficients $C(S, L)$ (typically found in the literature), and $\tilde{M}$ contains the reduced matrix elements in the pure $L-S$ coupling scheme. These are computed by the following formulas ${ }^{79}$

$$
\begin{aligned}
& \left\langle f^{n}[S L] J\|\vec{L}+2 \vec{S}\| f^{n}[S L] J\right\rangle=[(2 J+1)(J+1) / 4 J]^{1 / 2}[S(S+1)-L(L+1)+3 J(J+1)] \\
& \left\langle f^{n}[S L] J\|\vec{L}+2 \vec{S}\| f^{n}[S L] J-1\right\rangle=\left(\left[(S+L+1)^{2}-J^{2} \mathbb{J}^{2}-(L-S)^{2}\right] 4 J\right)^{/ 2} \\
& \left.\left\langle f^{n}[S L] J\|\vec{L}+2 \vec{S}\| f^{n}[S L] J+1\right\rangle=\left((S+L+1)^{2}-(J+1)^{2} \mathbb{T}(J+1)^{2}-(L-S)^{2}\right] 4(J+1)\right)^{/ 2}
\end{aligned}
$$

All other matrix elements are zero. The line strengths follow from (2.68). The following Tables contain the matrix $\tilde{S}^{M D}=(\hbar / 2 m c)^{2}\left|\tilde{M}^{\prime}\right|^{2}$ for various transitions in different rareearths $\left(\mathrm{Pr}^{3+}, \mathrm{Er}^{3+}, \mathrm{Dy}^{3+}, \mathrm{Tm}^{3+}, \mathrm{Nd}^{3+}, \mathrm{Ho}^{3+}\right.$, and $\left.\mathrm{Tb}^{3+}\right)$. These values are independent of host. The diagonal elements are purely mathematical and have no physical significance. 
Table 9.1. Magnetic dipole line strength matrix for various transitions in $\operatorname{Pr}^{3+}$. The coupling coefficients are taken from the energy eigenvectors in Ref. [80]. $S^{\mathrm{MD}}$ values are given in units of $10^{-20} \mathrm{~cm}^{2}$.

\begin{tabular}{|c|c|c|c|c|c|c|c|c|c|c|}
\hline$S^{M D}$ & ${ }^{3} \mathrm{H}_{4}$ & ${ }^{3} \mathrm{H}_{5}$ & ${ }^{3} \mathrm{H}_{6}$ & ${ }^{3} \mathrm{~F}_{2}$ & ${ }^{3} F_{3}$ & ${ }^{3} F_{4}$ & ${ }^{1} \mathbf{G}_{4}$ & ${ }^{1} D_{2}$ & ${ }^{1} \mathrm{I}_{6}$ & ${ }^{3} \mathbf{P}_{2}$ \\
\hline 3. & 116.66 & 10.538 & 0 & 0 & 0.0054 & 0.1261 & 0.0677 & 0 & 0 & 0 \\
\hline & 10.536 & 352.37 & 0 & 0 & 0 & 0.1156 & 0.1471 & 0 & 0 & 0 \\
\hline & 0 & 10.801 & 742.55 & 0 & 0 & 0 & 0 & 0 & 0.0443 & 0 \\
\hline 2 & 0 & 0 & 0 & 13.631 & 0 & 0 & 0 & 0.0743 & 0 & 0.0003 \\
\hline 3 & 0.0000 & 0 & 0 & 6.5202 & 98.583 & 0 & 0 & 0.1406 & 0 & 0.0056 \\
\hline 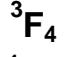 & 0.1262 & 0.1157 & 0 & 0 & 4.9275 & 250.79 & 2.3082 & 0 & 0 & 0 \\
\hline${ }^{1} \mathbf{G}_{4}$ & 0.0677 & 0.1471 & 0 & 0 & 1.8168 & 2.308 & 204 & 0 & 0 & 0 \\
\hline${ }^{1} D_{2}$ & 0 & 0 & 0 & 0.0742 & 0 & 0 & 0 & 32.002 & 0 & 0.5614 \\
\hline${ }^{1} I_{6}$ & 0 & 0.0317 & 0.0443 & 0 & 0 & 0 & 0 & 0 & 546.53 & 0 \\
\hline${ }^{3} P_{2}$ & 0 & 0 & 0 & 0.0003 & 0 & 0 & 0 & 0.5617 & 0 & 63.928 \\
\hline
\end{tabular}

